\title{
Dissecting the $\beta$-catenin-dependent and -independent functions of BCL9 and BCL9-2 in intestinal tumorigenesis
}

\author{
Doctoral Thesis \\ In partial fulfillment of the requirements for the degree \\ “Doctor rerum naturalium (Dr. rer. nat.)" \\ in the Molecular Medicine Study Program \\ at the Georg-August University Göttingen
}

submitted by

Maria Wiese

from

Halberstadt, Germany

Göttingen, December 2012 


\section{Members of the Thesis Committee}

\section{Supervisor}

Prof. Dr. Felix H. Brembeck

Hematology/Oncology/Tumor Biology and Signal Transduction

University Medical Center Göttingen

Second Member of the Thesis Committee

Prof. Dr. Matthias Dobbelstein

Molecular Oncology

University Medical Center Göttingen

Third Member of the Thesis Committee

Prof. Dr. Uwe-Karsten Hanisch

Neuropathology

University Medical Center Göttingen

Date of Disputation: 


\section{AFFIDAVIT}

Here I declare that my doctoral thesis entitled

"Dissecting the B-catenin-dependent and -independent functions of BCL9 and BCL9-2 in intestinal tumorigenesis"

has been written independently with no other sources and aids than quoted.

Göttingen, 17.12.12

(Maria Wiese) 


\section{Table of Contents}

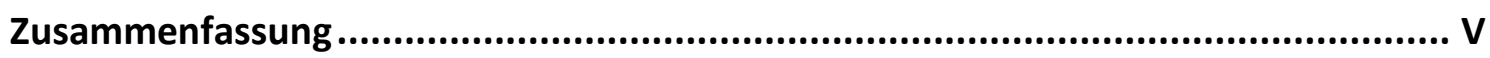

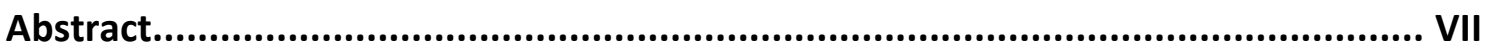

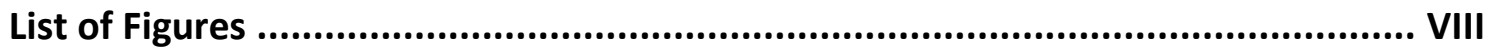

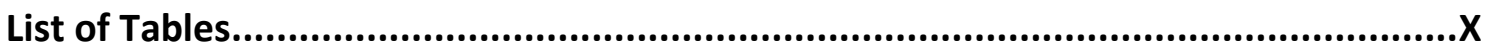

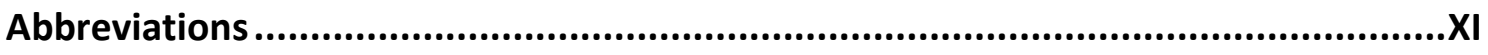

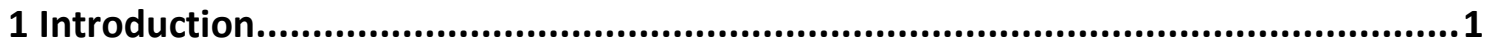

1.1 The Wnt/B-catenin-signaling pathway in intestinal development, homeostasis and

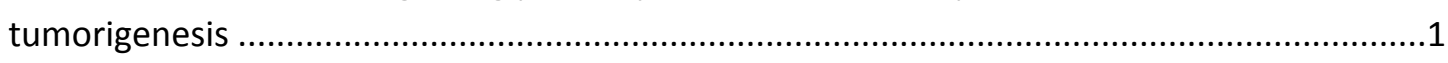

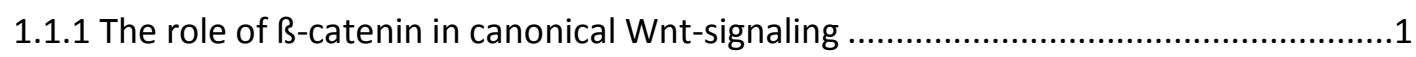

1.1.2 The $\mathrm{Wnt} /$ ß-catenin signaling pathway in intestinal development ..............................3

1.1.3 The Wnt/ß-catenin-signaling pathway and ß-catenin target genes in intestinal

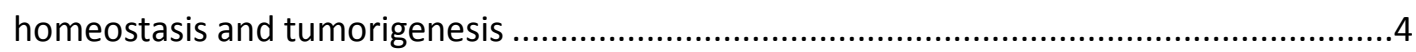

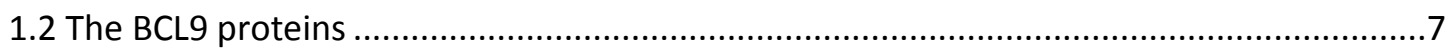

1.2.1 BCL9 and BCL9-2 encode the vertebrate orthologs of Drosophila Legless ...................7

1.2.2 The BCL9/Legless proteins are co-factors of the $\mathrm{Wnt} / \mathrm{B}$-catenin signaling pathway....8

1.2.3 BCL9 proteins in development, regeneration and tumorigenesis ..............................9

1.3. K19 dependent BCL9-2 overexpression in vivo ............................................................. 11

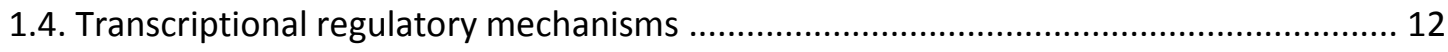

1.4.1 Basal transcriptional regulatory mechanisms......................................................... 12

1.4.2 Mechanisms of ß-catenin dependent transcriptional regulation of Wnt/ß-catenin

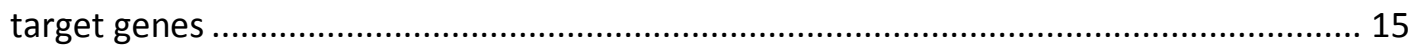

1.4.3. Crosstalk of LEF/TCF and SP1 in the transcriptional control of target gene expression

1.4.4 Transcriptional regulatory mechanism of Wnt/B-catenin target genes by the BCL9

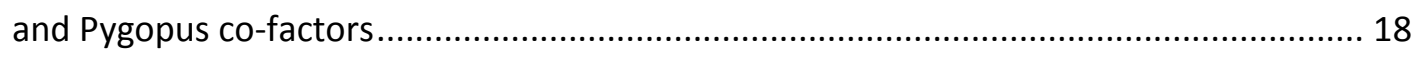

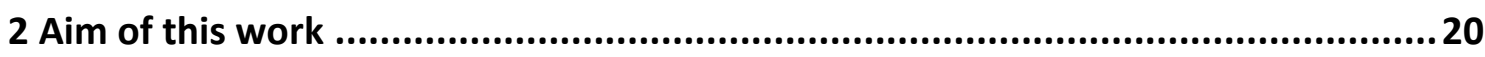

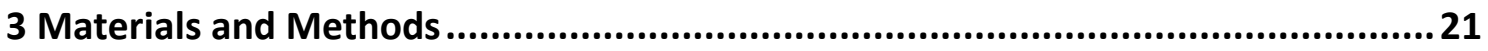

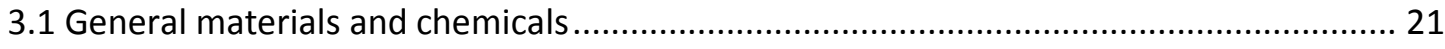

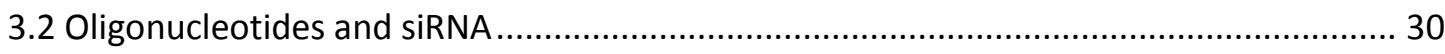

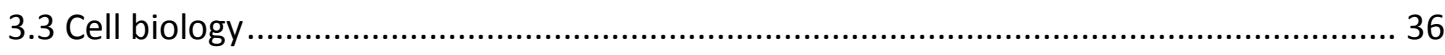

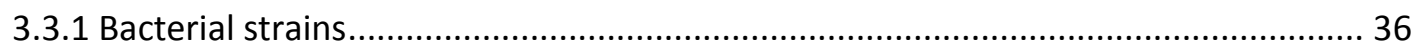

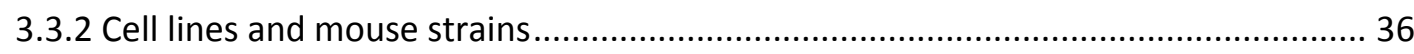

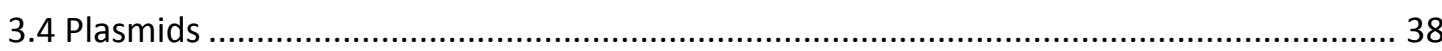


3.5 Buffers and solutions 39

3.5 Software 55

3.6 Microbiology 40

3.6.1 Cultivation and storage of $E$. coli 40

3.6.2 Generation of chemo-competent $E$. coli ........................................................ 40

3.6.2 Transformation of $E$. coli ....................................................................... 40

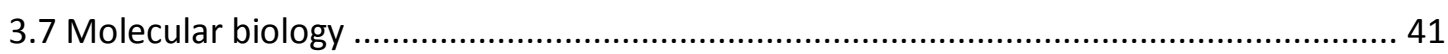

3.7.1 Isolation of nucleic acids ...................................................................... 41

3.7.2 Purification and extraction of nucleic acids .................................................. 42

2.7.3 Amplification of nucleic acids ............................................................ 42

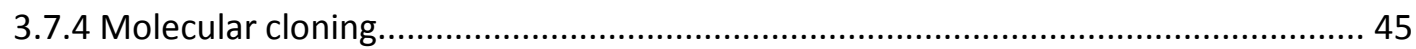

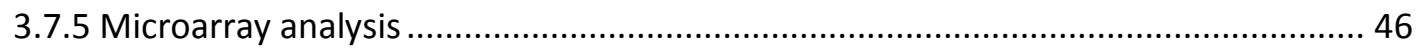

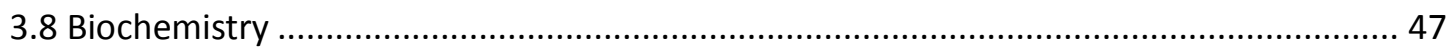

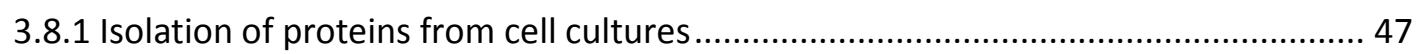

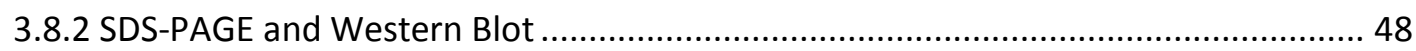

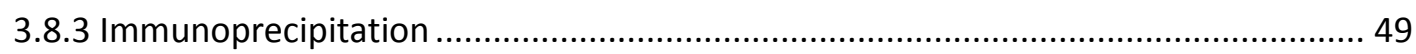

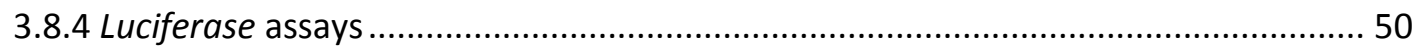

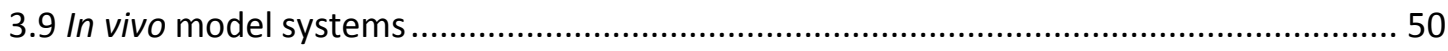

3.9.1 Mouse model systems ..................................................................... 50

3.9.2 Tissue processing for immunohistochemistry and Immunofluorescence ............... 51

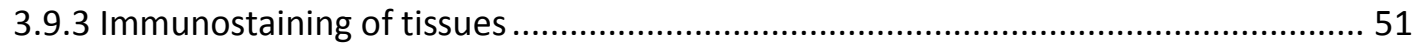

3.9.4 Scoring of human tissue microarray ........................................................... 53

3.9.5 Cell culture model systems ...................................................................... 53

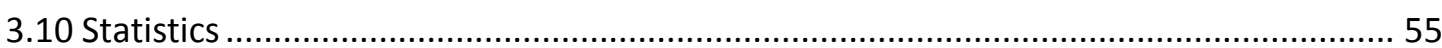

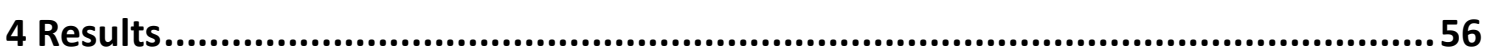

4.1 BCL9 and BCL9-2 expression in normal intestine and during intestinal tumorigenesis ... 56

4.1.1 BCL9-2 expression is restricted to the villi of the normal intestine and is up-regulated in early stages of intestinal tumorigenesis ................................................................. 56

4.1.2 BCL9-2, but not BCL9, is highly expressed in human colon cancers ....................... 57

4.2 BCL9-2 overexpression promotes intestinal tumor development................................ 59

4.2.1 BCL9-2 expression in compound $\mathrm{APC}^{\mathrm{Min} /+}$; K19-BCL9-2 transgenic mice .................. 59

4.2.2 BCL9-2 overexpression promotes tumor formation and local invasion .................. 61

4.3 BCL9 and BCL9-2 protein expression in colon cancer cell lines ................................63 63

4.4 Analysis of the dependency of the transcriptional control of ß-catenin, BCL9 and BCL9-2 on RNA Interference 
4.5 BCL9 and BCL9-2 knockdown reverts the mesenchymal malignant phenotype of cancer cell lines.

4.6 BCL9 and BCL9-2 are not target genes of ß-catenin

4.7 ß-catenin/Wnt-signaling activity correlates with the expression level of BCL9-2 in human colon cancer cells 68

4.8 BCL9-2 regulates ß-catenin-dependent and -independent target genes 71

4.8.1 BCL9-2 is not required for the expression of all canonical Wnt target genes and regulates $\beta$-catenin independent genes in colon cancer cells

4.8.2 B-catenin-dependent and -independent BCL9-2 target genes are expressed at the invasive front of adenomas of compound K19-BCL9-2; $\mathrm{APC}^{\mathrm{Min} / \mathrm{H}}$ mice ...

4.9 Whole genome microarray analyses demonstrate that BCL9, BCL9-2 and ß-catenin activate different gene expression profiles

4.9.1 B-catenin and BCL9/BCL9-2 induce different gene sets in cancer cells

4.10 Novel BCL9-2 target genes in cancer cells identified by microarray analyses

4.10.1 Comparison of the gene expression profile of cancer cell lines and of tumors from the intestine specific double knock out of VilCre;BCL9 ${ }^{-1} / \mathrm{BCL}^{-1-}{ }^{-/}$mice.

4.10.2 Cyclopholin A (СурA) and the stem-cell-derived neural stem/progenitor cell supporting factor (SDNSF) are newly identified BCL9-2 core target genes in cancer cell lines

4.11 Dissecting the ß-catenin-dependent and -independent functions of BCL9-2

4.11.1 BCL9-2 regulates the endogenous expression of caudal type homeobox 1 and 2 (CDX1/2)

5.11.2 Transcriptional activation by the proximal promoters of the homeodomain transcription factors $C D X 1$ and $C D X 2$ requires $B C L 9-2$, but not $\beta$-catenin in colon cancer cell lines.

4.11.3 Identification of putative transcription factor binding sites in the proximal promoters of CDX1 and CDX2

4.11.4 BCL9-2 modulates CDX1 and CDX2 reporter activity by Specificity Protein 1 (SP1)binding elements in their proximal promoter in colon cancer cell lines

4.12 BCL9-2 interacts with the transcription factor SP1 in colon cancer cell lines 93

5 Discussion. 95

5.1 The role of $\mathrm{BCL} 9$ and $\mathrm{BCL9-2}$ in intestinal homeostasis

5.2 BCL9-2 is up-regulated independently of Wnt/B-catenin signaling in early stages of intestinal tumorigenesis

5.3 BCL9-2 promotes intestinal tumorigenesis. 100

5.3.1 BCL9-2 expression enhances Wnt/ß-catenin signaling activity in intestinal tumorigenesis 
5.3.2 BCL9-2 is not required for the expression of all canonical Wnt target genes and regulates additional ß-catenin-independent genes implicated in tumorigenesis 102

5.3.3 BCL9-2 promotes tumor development and local invasion 107

5.4 A novel mechanism for BCL9-2 to regulate target gene transcription independently of $\mathrm{B}$ catenin

5.5.1 BCL9-2 regulates the expression of CDX1 and CDX2 independently of ß-catenin in colon cancer cells.

5.5.2 Identification of a new OCT4 responsive, transcription factor binding element in the proximal promoters of $\mathrm{CDX} 1$ and $\mathrm{CDX} 2$

5.5.3 CDX1 and CDX2 expression is regulated by BCL9-2 through newly identified SP1 binding elements in their proximal promoter

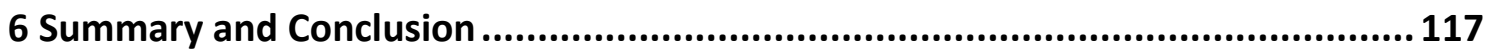

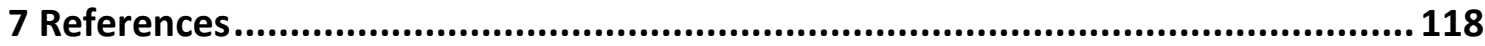

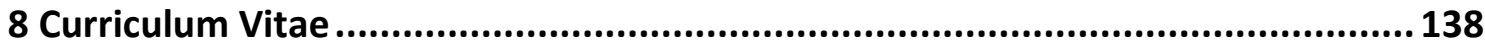




\section{Zusammenfassung}

Der hochkonservierte Wnt/ß-Catenin-Signaltransduktionsweg spielt eine wichtige Rolle während der Embryonalentwicklung, der Homöostase und der Tumorgenese in Adulten. Die BCL9 Proteine wurden zunächst als Kofaktoren dieses Signalweges identifiziert. Entsprechend agiert BCL9/Legless als essentieller Wnt/B-Catenin-Kofaktor in Drosophila. Jedoch scheint die Rolle von BCL9 und BCL9-2, der Orthologe von Legless, in Vertebraten komplexer zu sein. Des Weiteren wurden die genauen Funktionen der BCL9 Proteine während der intestinalen Homöostase und Tumorgenese bislang wenig untersucht. Es konnte jedoch bereits gezeigt werden, dass BCL9-2 in intestinalen und Mammakarzinomen verstärkt expremiert wird.

Diese Arbeit beschreibt erstmalig den Einfluss und die Funktion von BCL9-2 während der intestinalen Tumorgenese. BCL9-2 beinflusst die Tumorprogression positiv durch Verstärkung des Wnt/ß-Catenin-Signaltransduktionsweg und der Expression von Zielgenen, die Tumorwachstum und -invasion vermitteln. Zudem aktiviert BCL9-2 die Transkription von ßCatenin-unabhängigen Genen durch einen neuartigen Mechanismus.

Im Gegensatz zu BCL9, welches in allen humanen und murinen intestinalen Zelltypen expremiert wurde, beschränkte sich die BCL9-2 Expression auf die Zotten des Darmes. Die Wnt/B-Catenin-positiven Krypten hingegen zeigten keinerlei BCL9-2-Expression auf, was darauf hinweist, dass BCL9-2 für den Wnt/B-Catenin-Signalweg bei der intestinalen Homöostase entbehrlich ist. Während jedoch BCL9 Proteinlevel in Kolontumoren, im Vergleich zum normalen Epithel, unverändert blieben, wurde BCL9-2 bereits in frühen Stadien der Tumorgenese und in $90 \%$ aller Kolonkarzinome stark expremiert. Darüber hinaus führte transgene Überexpression von BCL9-2 im Darm von K19-BCL9-2;APC ${ }^{\mathrm{Min} /+}$ Mäusen zu einer verstärkten Formation von Adenomen, deren Invasion und einem verringerten Überleben der Versuchstiere.

Wie anhand von TOP/FOP Luciferase Reportergen-Versuchen gezeigt werden konnte, korrelierte die Stärke der BCL9-2-Proteinexpression mit der Aktivität des Wnt/ß-CateninSignalweges in Kolonkarzinomzellen. Zudem regulierte BCL9-2 die Transkription einiger BCatenin-abhängiger und darüber hinaus ß-Catenin-unabhängiger Zielgene, die bei der Tumorentstehung eine wichtige Rolle spielen. Des Weiteren zeigt diese Arbeit, dass in Kolonkarzinomzellen die BCL9-2 abhängige Transkription von CDX1 und CDX2 durch SP1bindende Elemente über deren proximale Promotoren vermittelt wurde. Mittels Immunpräzipitation konnte zudem eine Interaktion zwischen BCL9-2 und SP1 in Kolonkarzinomzellen bestätigt werden.

Zusammenfassend zeigt diese Arbeit, dass BCL9-2-Überexpression in frühen Phasen der intestinalen Tumorgenese die Progression von benignen Tumoren in invasive Karzinome 
fördert. Diese Eigenschaft wird durch verschiedene Mechanismen vermittelt: Zum einen verstärkt BCL9-2 die Expression einiger Wnt/B-Catenin-abhängiger Zielgene; zum anderen reguliert BCL9-2 ß-Catenin-unabhängige Gene, die für die Tumorgenese eine wichtige Rolle spielen. Diese Funktion wird vermutlich durch die Bindung an SP1 Transkriptionsfaktoren und damit an die Promotoren von BCL9-2 Zielgenen vermittelt, was zu der verstärkten Expression von Genen führt, die die Tumorprogression und Invasion fördern. 


\section{Abstract}

The Wnt/ß-catenin signaling pathway is highly conserved and plays an important role during embryonic development, in adult homeostasis and tumorigenesis. The members of the novel BCL9 family were characterized as co-activators of canonical Wnt-signaling. BCL9/Legless was found to be absolutely essential for Wnt/B-catenin signaling in Drosophila. However, the role of BCL9 and the second homolog, BCL9-2, seems to be more complex in vertebrates. BCL9-2 was shown to be overexpressed in colon and breast cancers. However, detailed analyses of the BCL9 proteins and their function in normal intestines and during the multistep model of carcinogenesis were missing. Here, we show that BCL9-2 promotes intestinal tumor progression through the enhancement of $\mathrm{Wnt} / ß$-catenin signaling and the regulation of target genes which trigger tumor growth and invasion. Moreover, we demonstrate that BCL9-2 activates the transcription of ß-catenin-independent genes by a novel mechanism.

Using Immunohistochemistry BCL9 was found to be expressed in all intestinal cell types and unchanged in colon cancer cells. In contrast, BCL9-2 protein expression was restricted to the villi in normal intestines, and absent in the crypts where Wnt-signaling is active, indicating that BCL9-2 is dispensable for Wnt/ß-catenin signaling in intestinal homeostasis. In addition, we found that BCL9-2 overexpression occurs early during intestinal tumorigenesis and is overexpressed in approximately $90 \%$ of human adenocarcinomas. Moreover, transgenic overexpression of BCL9-2 in the intestine of transgenic K19-BCL9-2;APC ${ }^{\mathrm{Min} /+}$ mice led to increased adenoma formation accompanied with local invasion which resulted in reduced survival.

Using TOP/FOP Luciferase reporter-gene assays, we found that BCL9-2 protein expression correlated with the level of Wnt/ß-catenin signaling activity in colon cancer cells. Moreover, BCL9-2 regulated the transcription of a subset of ß-catenin-target genes. In addition, we identified a set of BCL9-2 target genes which was apparently independent of ß-catenin signaling which have been implicated in tumorigenesis. Here, we demonstrate that the activation of CDX1 and CDX2 gene transcription by BCL9-2 was dependent on SP1-binding elements in their proximal promoters in colon cancer cell lines. This was corroborated by the novel finding that BCL9-2 associated with the specific protein 1 (SP1) in cancer cells.

In conclusion, this work demonstrates that BCL9-2 overexpression promotes early phases of intestinal tumorigenesis and contributes to the progression of tumors into invasive carcinomas. We showed that BCL9-2 enhances the activation of certain Wnt/B-catenin target genes. Moreover, BCL9-2 regulates its own set of target genes apparently independent of ßcatenin. This function is mediated through binding to SP1 transcription factors and thereby to the promoters of BCL9-2 target genes, which results in aberrant expression of proteins that trigger the promotion of tumor progression and invasion. 


\section{List of Figures}

Figure 1: Schematic overview of the Wnt/ß-catenin-signaling pathway.......................................

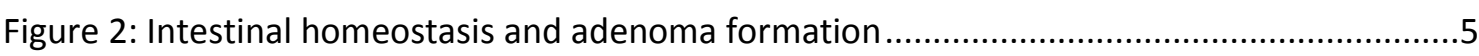

Figure 3: Gene mutations during the adenoma-carcinoma sequence ......................................6

Figure 4: Schematic overview of the BCL9 proteins including seven evolutionary conserved domains

Figure 5: Transcriptional enhancers cooperating with the pre-initiation complex and initiating gene transcription through a variety of core promoters.

Figure 6: Schematic overview about the protein structure and modification motifs of SP1 and SP3.

Figure 7: Transcriptional regulation of Wnt/ß-catenin target genes..................................... 16

Figure 8: BCL9/Pygopus dependent transcriptional activation of Wnt/B-catenin target genes 18

Figure 9: Scheme of mutagenesis PCR to indroduce mutations in the promoters of CDX1 and

CDX2.

Figure 10: Expression of BCL9 and BCL9-2 in normal embryonic and adult intestines and in adenomas

Figure 11: BCL9-2 is up-regulated in human adenocarcinomas

Figure 12: Transgenic BCL9-2 expression in compound $A P C^{\mathrm{Min} /+} ; \mathrm{K} 19-\mathrm{BCL} 9-2$ intestinal

adenomas and adjacent normal tissues compared to non-transgenic $\mathrm{APC}^{\mathrm{Min} /+}$ littermate controls

Figure 13: BCL9-2 overexpression leads to impaired survival of compound $\mathrm{APC}^{\mathrm{Min} /+}$; K19-BCL9-2

mice.

Figure 14: BCL9-2 overexpression promotes adenoma formation in number and size in compound APCMin/+; K19-BCL9-2 mice

Figure 15: BCL9-2 overexpression induced invasion in compound $\mathrm{APC}^{\mathrm{Min} /+}$; K19-BCL9-2 mice . 63

Figure 16: Expression of BCL9 proteins in different colon cancer cell lines

Figure 17: Knockdown efficiency of 4 single and pooled siRNAs targeting BCL9, BCL9-2 and Bcatenin in SW480 cells

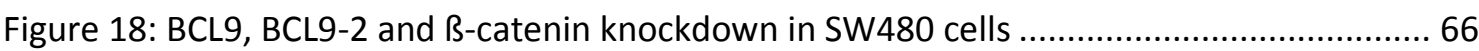

Figure 19: Knockdown of BCL9 and BCL9-2 induces characteristic morphological changes ...... 67

Figure 20: BCL9 and BCL9-2 are not target genes of $ß$-catenin

Figure 21: BCL9-2 expression levels correlates with canonical Wnt signaling activity and reporter gene expression in different colon cancer cell lines.

Figure 22: BCL9-2 regulated its own subset of target genes, apparently independent of ßcatenin.

Figure 23: Expression of BCL9-2 targets in invasive areas of compound K19-BCL9-2; APC $\mathrm{Min} /+$ mice.

Figure 24: ß-catenin and BCL9/BCL9-2 induce different gene sets in cancer cell lines .............. 75

Figure 25: $B C L 9-2$ regulates target genes independently of $ß$-catenin.

Figure 26: Comparative gene expression profiles from VilCre;BCL9 ${ }^{-/} / \mathrm{BCL9}-2^{-/-}$and microarrays from siRNA treated SW480 cells identified new target genes of the BCL9 proteins.................. 78

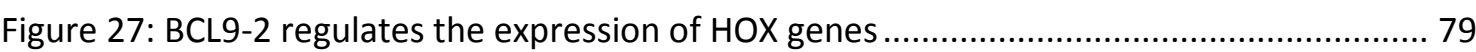

Figure 28: Microarray analysis identified 2 genes as new BCL9-2 core target genes in cancer cell lines 
Figure 29: $B C L 9-2$ but not $B C L 9$ and B-catenin regulates the mRNA and protein expression of Cyclophilin A (СурA) and stem-cell-derived neural stem/progenitor cell supporting factor (SDNSF) in cancer cell lines.

Figure 30: The Wnt/ß-catenin target gene CDX1 and the ß-catenin-independent gene CDX2 are regulated by BCL9-2 in colon cancer cell lines

Figure 31: BCL9-2, but not ß-catenin knockdown reduces the activity of CDX1 and CDX2 Luciferase-reporters. 84

Figure 32: Transcription factor binding elements in the CDX1 and CDX2 proximal promoter... 87 Figure 33: BCL9-2 regulates CDX1 reporter gene expression by an SP1 responsive element in the proximal promoter.

Figure 34: SP1 binding elements mediate the BCL9-2 dependent regulation of the CDX2 promoter

Figure 35: OCT4 induces the CDX1 and CDX2 reporter activities through a newly identified OCT4 binding element .92

Figure 36: Co-Immunoprecipitation identified SP1 as a new interaction partner of BCL9-2 …. 93

Figure 37: BCL9-2 expression and $\mathrm{Wnt} / \mathrm{B}$-catenin signaling in the intestine 98

Figure 38: BCL9-2 and BCL9 regulate a subset of $\mathrm{Wnt} / \mathrm{B}$-catenin target genes and regulate the expression of distinct genes independent of ß-catenin 103

Figure 39: Overview about putative mechanisms for BCL9-2/OCT4 transcriptional activation of CDX2 112

Figure 40: BCL9-2 regulates canonical Wnt-target genes and ß-catenin independent genes. 116 


\section{List of Tables}

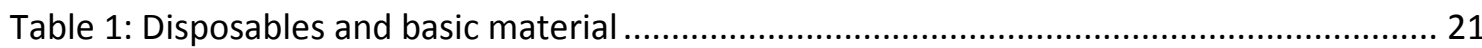

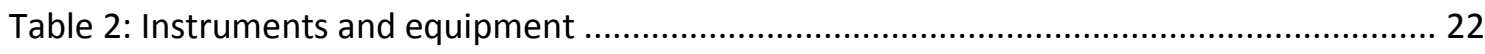

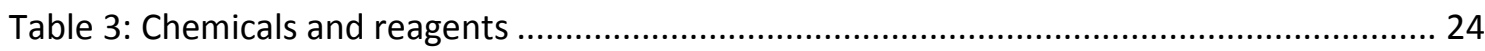

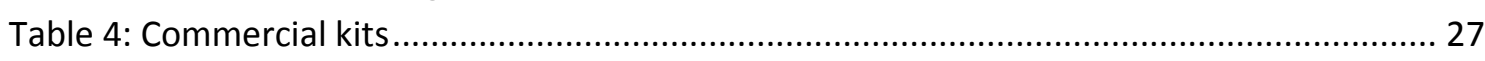

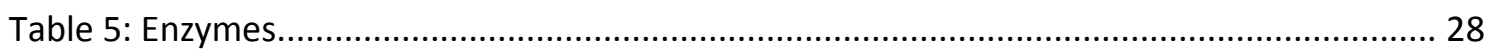

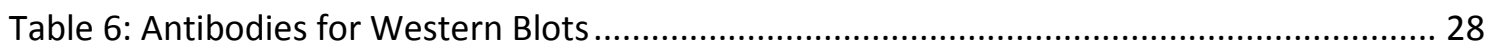

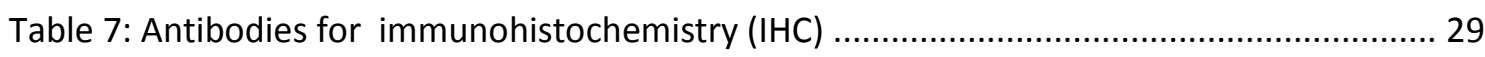

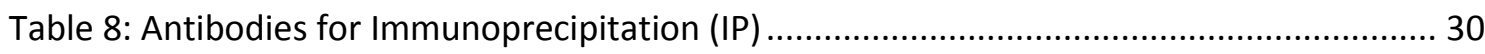

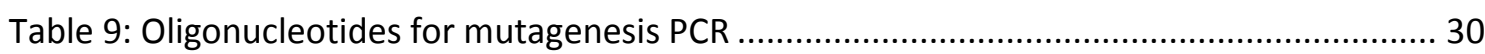

Table 10: Oligonucleotides for sequencing reactions of Luciferase constructs.......................... 32

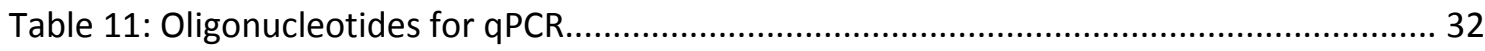

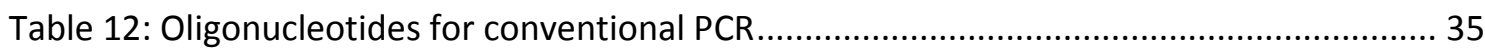

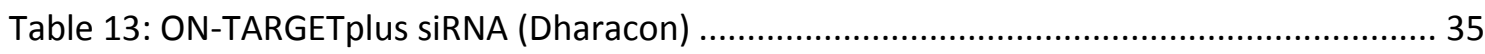

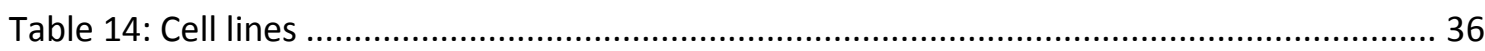

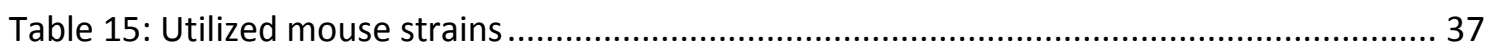

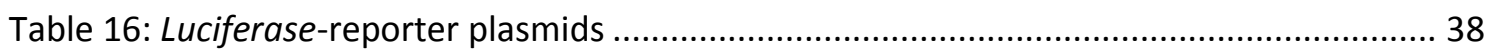

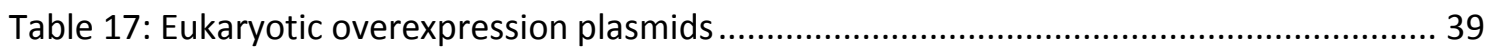

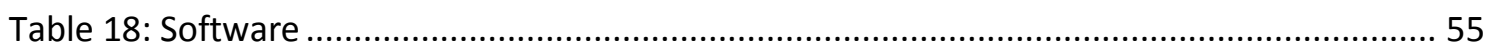

Table 19: Cells / $\mathrm{ml}$ used for transfection with siRNA and with overexpression-plasmids (oe) . 54

Table 20: Components of Lipofectamine based transfection mixes ......................................... 55 


\section{Abbreviations}

\begin{tabular}{|c|c|}
\hline$\mu l$ & micro \\
\hline A/LysoPLA & A/lyso-phospholipase \\
\hline aa & aminio acid \\
\hline Ac & Acetylation \\
\hline ACTB & ß-actin gene/mRNA \\
\hline APC & adenomatous polyposis coli \\
\hline bcatBD & ß-catenin binding domain \\
\hline BCL9 & B-cell CLL/lymphoma 9 protein \\
\hline BCL9-2/BCL9L & B-cell CLL/lymphoma 9-like protein \\
\hline BD & binding domain \\
\hline $\mathrm{BE}$ & binding elements \\
\hline BGH-polyA & bovine growth hormone polyadenylation \\
\hline BMP & bone morphogenic protein \\
\hline bp & base pairs \\
\hline BrdU & Bromodeoxyuridine \\
\hline BRE & TBIID recognition element \\
\hline BRG1 & brahma-related gene 1 \\
\hline CA1 & carbonic anhydrase 1 \\
\hline CBP & CREB-binding protein \\
\hline CCND1 & CyclinD1 gene/mRNA \\
\hline cDNA & complementary DNA \\
\hline CDX & caudal type homeobox \\
\hline C-HD & C-terminal homology domain \\
\hline $\mathrm{CIN}$ & chromosomal instability \\
\hline CIP & calf intestine phosphatase \\
\hline CK1 & casein kinase 1 \\
\hline CRC & colorectal carcinomas \\
\hline CREB & cAMP responsive element binding protein 1 \\
\hline CTNNB1 & ß-catenin gene/mRNA \\
\hline CypA & Cyclopholin A \\
\hline DAPI & 4',6-diamidino-2-phenylindole \\
\hline DKK1 & Dickkopf-related protein 1 \\
\hline DMSO & Dimetylsulfoxide \\
\hline
\end{tabular}




\begin{tabular}{|c|c|}
\hline DNA & Desoxyribonucleic acid \\
\hline dNTP & Desoxyribonucleotide triphosphate \\
\hline DPE & Downstream core promoter elements \\
\hline DSH & Dishevelled \\
\hline DSS & dextran sulfate sodium \\
\hline DTT & DL-Dithiothreitol \\
\hline $\mathrm{E}$ & embryonic day \\
\hline EDTA & Ethylenediaminetetraacetate \\
\hline EGTA & ethylene glycol tetraacetic acid \\
\hline EMT & epithelial-mesenchymal-transition \\
\hline EPH & Ephrin receptor \\
\hline ephrin/EFNB & ephrin ligand \\
\hline ER & Estrogen \\
\hline ER $\alpha$ & estrogen receptor alpha \\
\hline ESC & embryonic stem-cell \\
\hline ESR1 & Estrogen receptor alpha gene \\
\hline $\mathrm{EtBr}$ & ethidium bromide \\
\hline EtOH & Ethyl alcohol \\
\hline EZH2 & Enhancer of zeste (Drosophila) homolog 2 \\
\hline FACS & fluorescence activated cell sorting \\
\hline FBS & fetal bovine serum \\
\hline FDR & fold discovery rate \\
\hline FGF & fibroblast growth factor \\
\hline for & (primer) forward \\
\hline FZ & Frizzled \\
\hline GREB1 & Growth regulation by estrogen in breast cancer 1 \\
\hline GSK3ß & Glycogen synthase kinase $3 ß$ \\
\hline h & hours or human \\
\hline$H \& E$ & Hematoxylin \& Eosin \\
\hline $\mathrm{H} 2 \mathrm{O} 2$ & Peroxygen \\
\hline H3 & histone 3 \\
\hline HAT & histone acetyltransferases \\
\hline $\mathrm{HCl}$ & Hydrochloric acid \\
\hline HD & homology domain \\
\hline HDAC & histone deacetylases \\
\hline
\end{tabular}




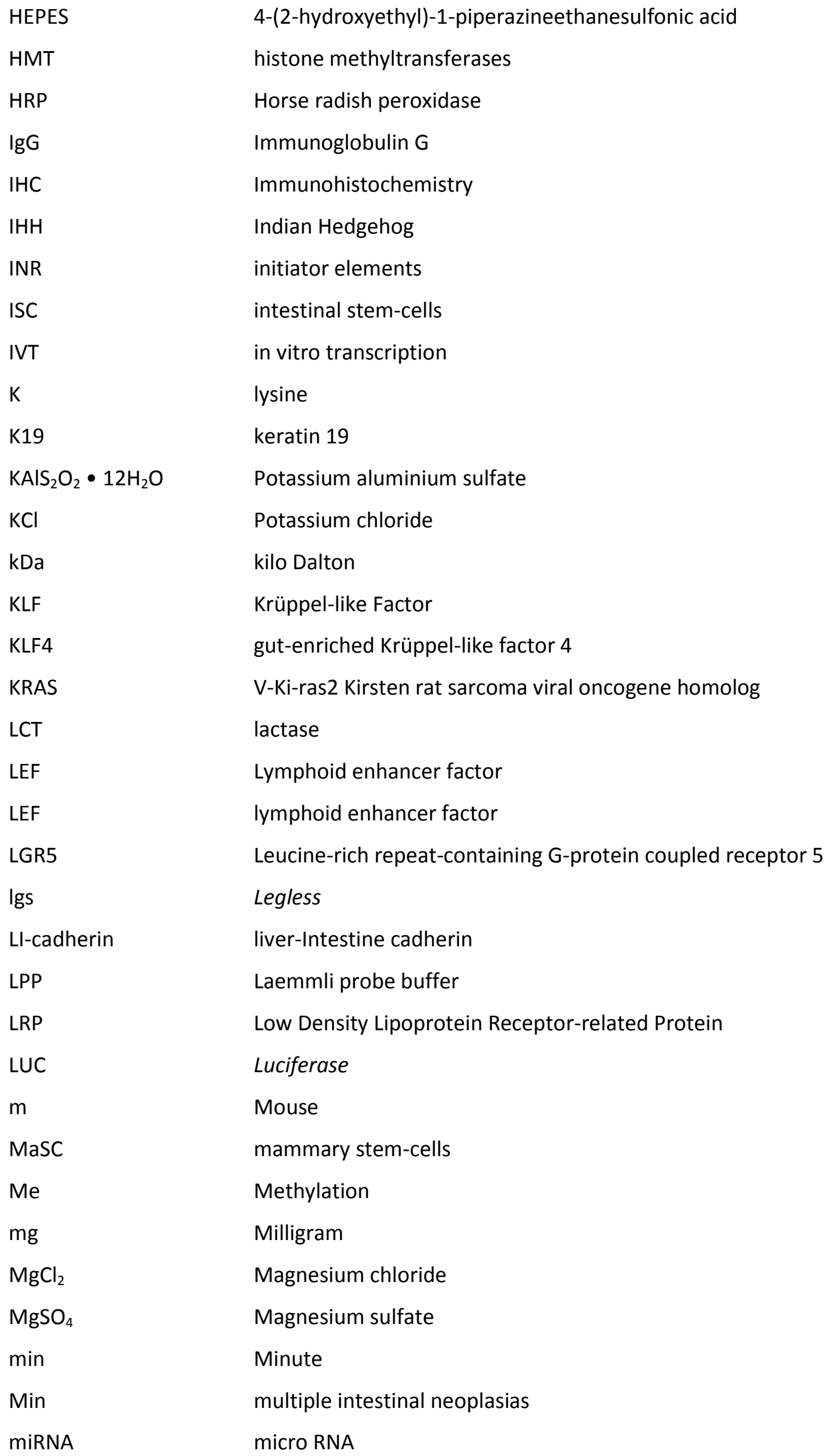




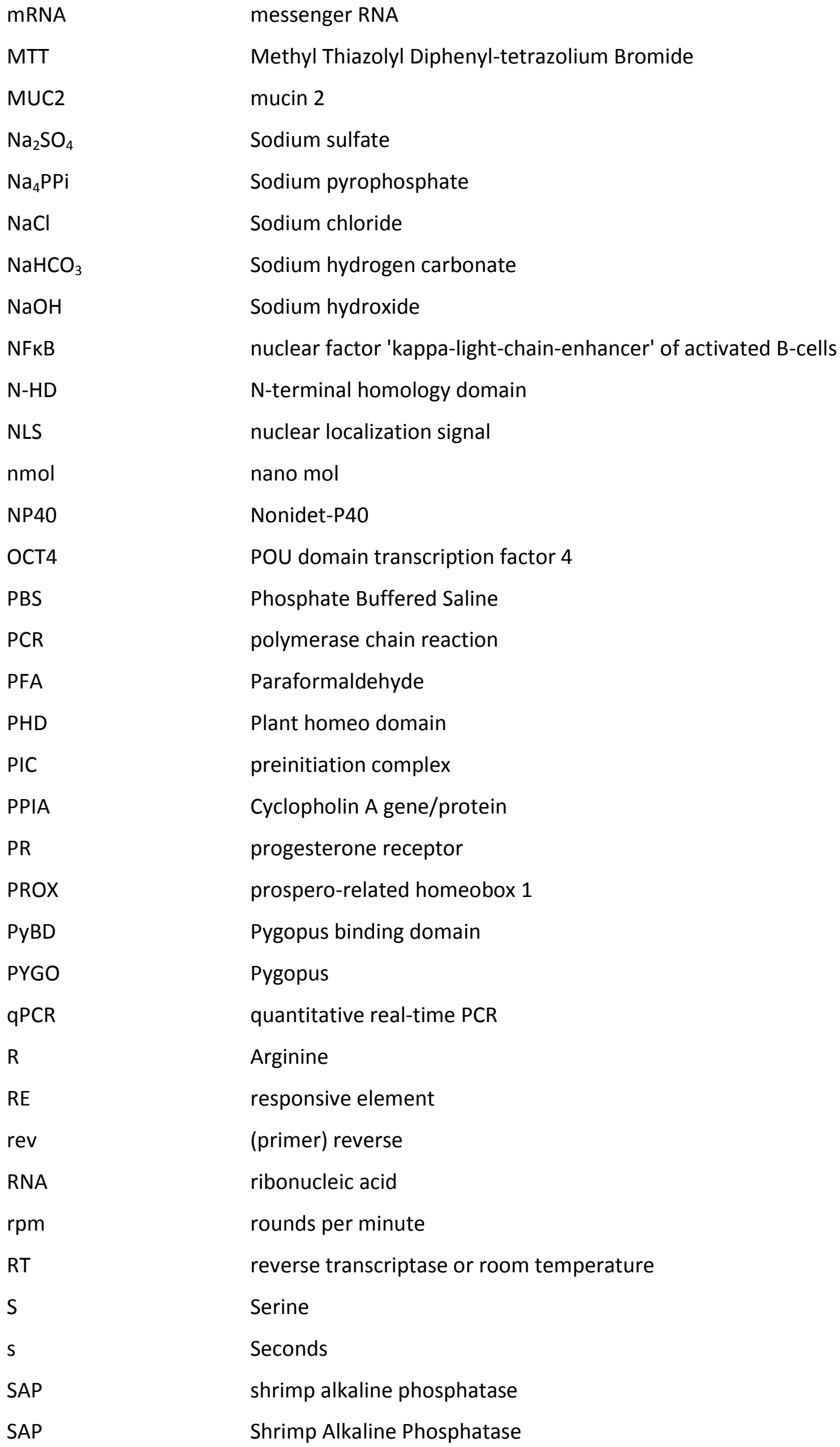

Sodium chloride

Sodium hydrogen carbonate

Sodium hydroxide

nuclear factor 'kappa-light-chain-enhancer' of activated B-cells

$\mathrm{N}$-terminal homology domain

nuclear localization signal

nano mol

Nonidet-P40

POU domain transcription factor 4

Phosphate Buffered Saline

polymerase chain reaction

Paraformaldehyde

Plant homeo domain

preinitiation complex

Cyclopholin A gene/protein

progesterone receptor

prospero-related homeobox 1

Pygopus binding domain

Pygopus

quantitative real-time PCR

Arginine

responsive element

(primer) reverse

ribonucleic acid

rounds per minute

reverse transcriptase or room temperature

Serine

Seconds

shrimp alkaline phosphatase

Shrimp Alkaline Phosphatase 
SDNSF

SDS

SDS-PAGE

SI

SiRNA

SMAD

SNAIL1

SNAIL2

SOX

SOX2

$\mathrm{SP} 1 / 3$

ß-TRCP

TA

TAL

TBP

TBST

TCF

TEMED

TF

TFIID

TG

TGFß

TS

UTR

VEGF

VIM

WRE

Y

ZEB

$\beta$-TRCP stem-cell-derived neural stem/progenitor cell supporting factor

Sodium Dodecyl Sulfate

SDS polyacrylamide gel electrophoresis

Sucrase isomaltase

short interfering RNA

sisters and mothers against decapentaplegic

Snail

Slug

SRY-related HMG-box gene

SRY (sex determining region Y)-box 2

specific protein $1 / 3$

ß-Transducing repeat containing protein

transient amplifying cells

"Transkritomanalyselabor"

TATA-binding proteins

Tris-Buffered Saline Tween-20

T cell factor

$\mathrm{N}, \mathrm{N}, \mathrm{N}^{\prime}, \mathrm{N}^{\prime}$-Tetramethylethan-1,2-diamin

transcription factor

transcription factor II D

Transgenic

Transforming growth factor

transcription start site

untranslated region

Vascular endothelial growth factor

Vimentin

Wnt responsive elements

Thyrosine

zinc finger E-box binding homeobox

beta-transducin repeat containing 


\section{Introduction}

\subsection{The Wnt/ß-catenin-signaling pathway in intestinal development, homeostasis and tumorigenesis}

Wnts are a family of secreted proteins which activate several signaling pathways through binding to Frizzled and therefore, inducing the canonical (Wnt/ß-catenin)-, the non-canonical (planar-cell-polarity)- and the Wnt-Ca ${ }^{2+}$ - pathway (Habas and Dawid, 2005).

The Wnt/ß-catenin signal transduction pathway is highly conserved between all animals (Logan and Nusse, 2004;Nusse et al., 1997;van and Clevers, 2002;Wodarz and Nusse, 1998). It plays an important role during embryonic development (Grigoryan et al., 2008;Logan et al., 2004;Niehrs, 2010) and adult tissue maintenance (Logan et al., 2004;Nusse et al., 2008;Polakis, 2012;van et al., 2002) by modulating different cellular processes including proliferation, survival, cell migration, differentiation and patterning. In addition, canonical Wnt-signaling is implicated in various diseases, in particular in tumorigenesis (Bienz and Clevers, 2000; Logan et al., 2004).

\subsubsection{The role of ß-catenin in canonical Wnt-signaling}

ß-catenin was first described as the segment polarity gene product, Armadillo, in Drosophila (Wieschaus and Riggleman, 1987). It harbors dual functions: as a component of adherence junctions it is necessary for regulating cell growth and adhesion (Brembeck et al., 2006) and in addition, as a modulator of transcription as the key component of canonical Wnt-signaling. In the absence of extracellular Wnt signals, cytoplasmic ß-catenin is recruited to the destruction complex which consists of AXIN1/2, Adenomatous-polyposis-coli (APC), Glycogen synthase kinase $3 ß$ (GSK3ß) and Casein kinase $1 \varepsilon$ (CK1ع) (Golan et al., 2004). ß-catenin is initially Nterminally phosphorylated by CK1 $1 \varepsilon$ at serine 45 (S45), followed by serine 33 (S33), serine 37 (S37) and tyrosine 41 (Y41) phosphorylation by GSK3 $\beta$ (Liu et al., 2002a). This phosphorylation primes $\beta$-catenin for $\beta$ Transducing repeat containing protein ( $\beta$-TRCP) mediated ubiquitination and its subsequent degradation by the proteasome (He et al., 2004a). Binding of extracellular Wnt ligands to the Frizzled (FZ) - lipoprotein receptor-related protein 5/6 (LRP5/6)-complex leads to activation of Dishevelled (DSH) and disassembly of the destruction complex. 
Consequently, ß-catenin is stabilized and trans-locates to the nucleus where it binds to lymphoid enhancer factor/T cell factor (LEF/TCF) transcription factors (Behrens et al., 1996). The function of $\beta$-catenin involves the recruitment of additional co-factors, including chromatin modifying and remodeling factors (Parker et al., 2002), p300/ CREB-binding protein (CBP) (Hecht et al., 2000), brahma-related gene 1 (BRG1) (Barker et al., 2001) and TATAbinding proteins (TBP) (Bauer et al., 1998; Hecht et al., 1999) (see 1.4).

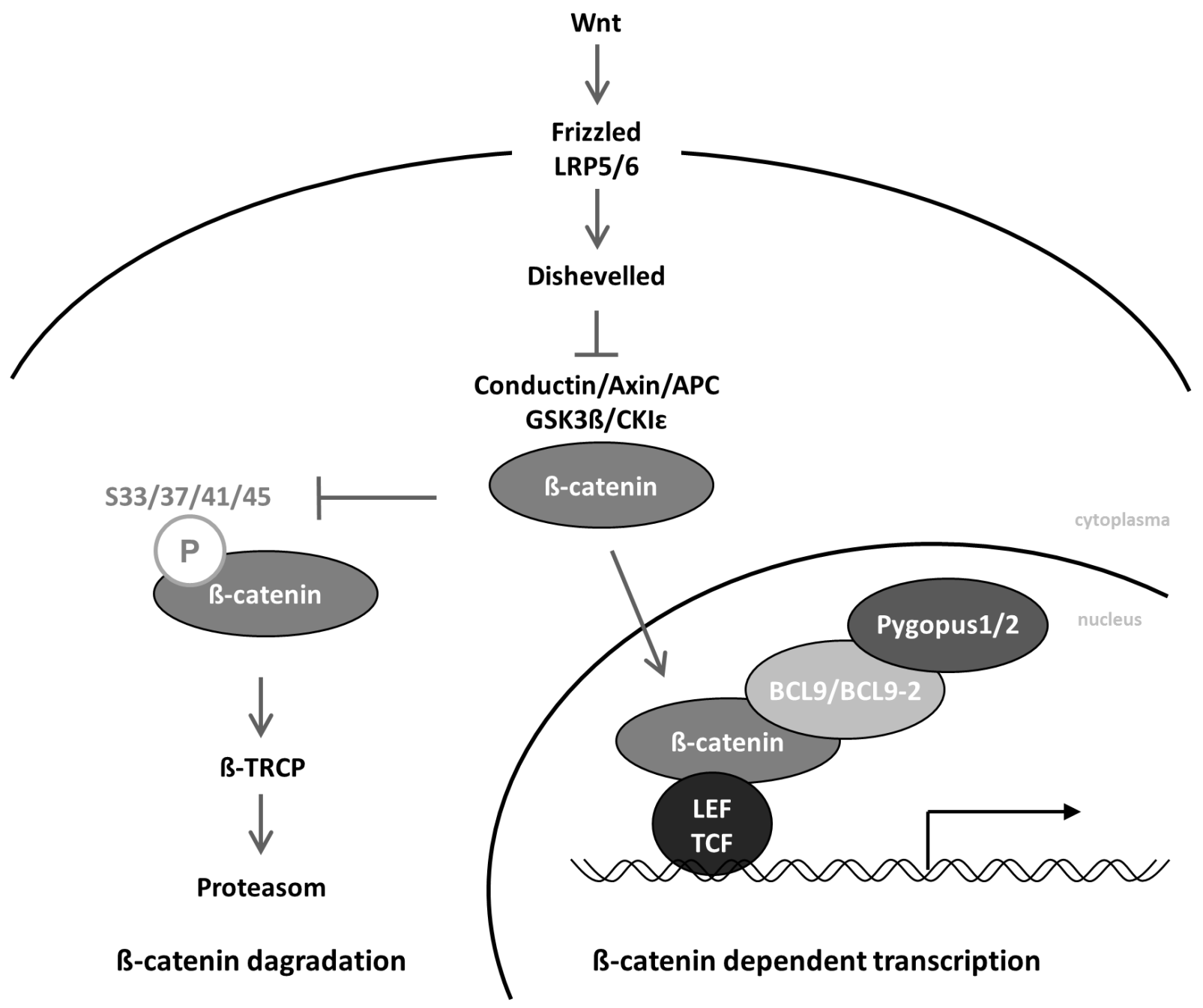

Figure 1: Schematic overview of the Wnt/ß-catenin-signaling pathway

Without extracellular stimuli, ß-catenin is phosphorylated by the destruction complex consisting of Axin1/2, APC, GSK3ß and CK1 1 , ubiquitinylated by $ß-T R C P$ and subsequently degraded by the proteasome. When Wnt signals bind to LRP/Fz receptors Dsh becomes activated which inhibits the destruction complex. Stabilized ß-catenin enters the nucleus and binds to LEF/TCF transcription factors and additional co-activators like the BCL9 and Pygopus proteins. This activates the transcription of $ß-$ catenin-dependent target genes. (adapted from Brembeck et al., 2006)

In addition, BCL9 proteins bind to ß-catenin and further recruit other co-activators like Pygopus1 and 2 which lead to enhanced ß-catenin target gene transcription (Belenkaya et al., 2002;Brembeck et al., 2004;Jessen et al., 2008) (Figure 1) (see 1.4.2 and 1.4.3). In the absence of stabilized ß-catenin, TCF transcription factors are bound to Groucho as well as histone 
deacetylases. This complex consequently acts as a transcriptional repressor of Wnt/ß-catenin target genes (Cavallo et al., 1998;Chen and Struhl, 1999;Daniels and Weis, 2005)(see 1.4.2).

\subsubsection{The Wnt/ß-catenin signaling pathway in intestinal development}

Development of the intestine occurs early during embryogenesis. In mice, during E10 to E13, the endodermal germ layer gives rise to the primitive gut tube, whose inner endodermal core differentiates to the epithelium of the mature gastrointestinal tract. After this transition the different specific intestinal cell types develop (see 1.1.3.). In the post-natal period the small intestinal villi elongate and the crypts are formed. The development of the colon is delayed relative to the small intestine. In addition, no villis are formed in the colon, while the crypts merge into a surface epithelial layer (Gao et al., 2009;Sherwood et al., 2011;van der Flier and Clevers, 2009;van der Flier et al., 2009).

These developmental processes require the expression of intestine-specific genes, which are regulated by the key-regulatory ParaHox genes. Those include the caudal related homeodomain proteins CDX1 and CDX2 (Gao et al., 2009). These core-transcription factors are responsible for the activation of general intestine specific genes, such as Mucin 2 (MUC2), gutenriched Krüppel-like factor 4 (KLF4) and Liver-Intestine cadherin (LI-cadherin), genes specific for the small intestine (e.g. sucrose isomaltase (SI), lactase (LCT) and A/lyso-phospholipase (A/LysoPLA)) and colonic epithelial genes (e.g. carbonic anhydrase 1 (CA1)) (Guo et al., 2004) through evolutionarily conserved DNA promoter elements (Suh et al., 1994). The activation of CDX1 and CDX2 target genes is highly dependent on the phosphorylation status of these proteins (Taylor et al., 1997). The initial expression of CDX2 is induced by canonical Wntsignaling between E7.5 and E8.5, while after E8.5 Wnt-signaling is no longer sufficient to activate CDX2 (Sherwood et al., 2011). CDX2 protein expression in the posterior endoderm induces the expression of further intestinal Hox genes, including CDX1 and as a target gene of CDX2 and ß-catenin expression (Mutoh et al., 2009; Lickert et al., 2009). CDX1 protein expression appears after a short delay compared to CDX2 at E9.5 predominantly in the distal part of the developing intestine. CDX1 and CDX2 expression become restricted to the intestinal epithelium. As a consequence, a sharp anterior boundary is formed marking the transition from stomach to duodenum (Hu et al., 1993;James et al., 1994). Thus, the gradients of CDX2 and CDX1 define the three major parts of the intestine: the foregut which gives rise to the epithelia of esophagus, stomach, and duodenum, the midgut which becomes the small intestine and the hindgut, which develops into the cecum and colon. A gradient of CDX1 and 
CDX2 forms the crypt-villus axis, with CDX1 expression primarily in the crypt and CDX2 in the villus region (Sherwood et al., 2011).

In addition, Wnt-signaling contributes to the proper development of the intestine by modulating key-epithelial signals which define the epithelial integrity to the underlying mesenchyme by regulating the expression of e.g. Indian Hedgehog (IHH) (Buller et al., 2012; Sherwood et al., 2011). According to this, TCF1/TCF4 mutants display severe embryonic intestinal defects (Gregorieff et al., 2004). In addition, ablation of ß-catenin in the node, notochord and anterior primitive streak abrogates definitive endoderm formation (Imai et al., 2000; Lickert and Kemler, 2002). In summary, development of the gastrointestinal tract is governed by the cooperation of different core-factors including ß-catenin, which activates the expression of canonical Wnt-target genes, and the major specification transcription factor CDX2.

\subsubsection{The Wnt/ß-catenin-signaling pathway and ß-catenin target genes in intestinal homeostasis and tumorigenesis}

The intestine is covered by a single layer of epithelial cells surrounding the finger-like villi. These invade into the crypts of Lieberkühn consisting of non-differentiated, proliferating cells (Figure2). The crypts harbor the intestinal stem-cells, which give rise to transient-amplifying cells that divide 4-5 times before they differentiate into the absorptive enterocytes, mucoussecreting goblet cells, lysozyme producing Paneth cells and hormone-secreting enteroendocrine cells. The structural organization of the intestine leads to an extreme enlarged surface. In addition, the cellular organization conveys the major functions of the small intestine including digestion and absorption of nutrients and the formation of a barrier against luminal pathogens. In contrast, the colon lacks the constitution of villi. Consequently, the main function of the colon is the absorption of water and thus the compaction of stool mass. (Sancho et al., 2003)

The bottom of the crypts consists of multipotent intestinal stem-cells (ISCs), which are bordered by single Paneth cells. The ISCs have a cell cycle period of $24 \mathrm{~h}$ and reside in the lower part of the crypt. They give rise to the transient amplifying (TA) cells which reside in the crypts for up to six cell divisions with a cell cycle period of around 12h (Marshman et al., 2002). When the TA-descendants reach the crypt-villus junction they undergo cell cycle arrest and differentiate into the four specific, epithelial intestinal cell types. While the intestinal cells differentiate they migrate along the crypt-villus axis. In mice the tip of the villus is reached by 
the cells 3 days after their terminal differentiation, where they undergo apoptosis and are shed into the lumen (Hall et al., 1994)(Figure 2). Paneth cells represent an exception. They move towards the bottom of the crypts upon differentiation and exhibit an enlarged life span of 20 days compared to the three other intestinal, epithelial, mature cell types (Garabedian et al., 1997).

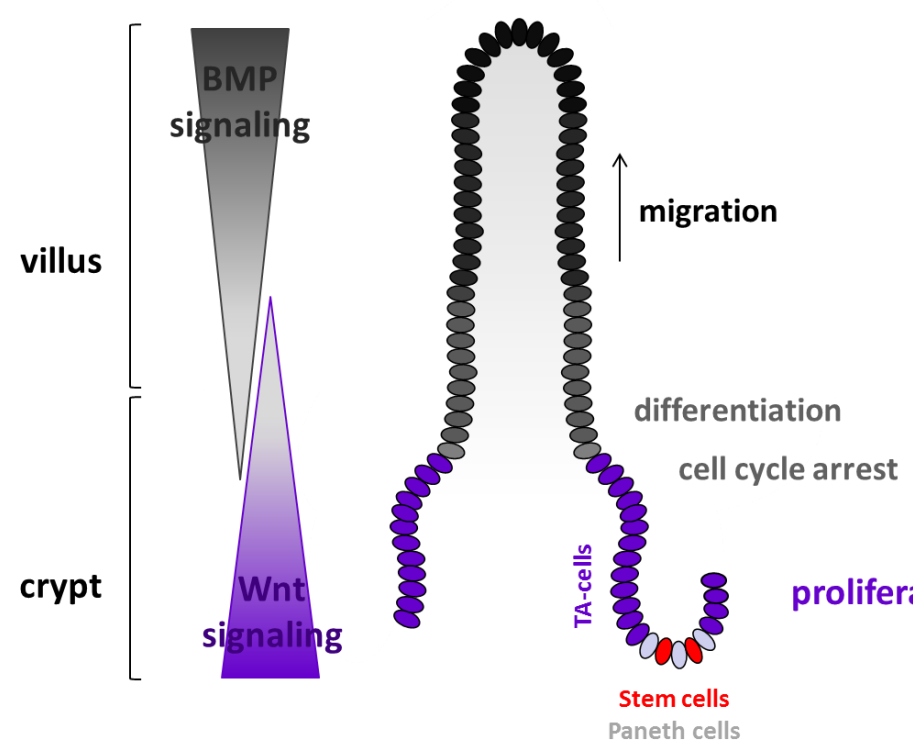

normal intestine

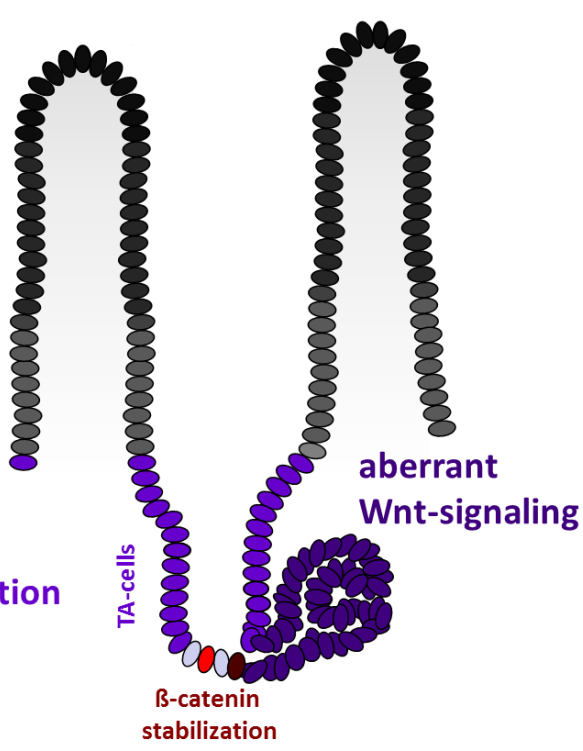

adenoma formation

Figure 2: Intestinal homeostasis and adenoma formation

Overview of the organization of the crypt-villus organization of the small intestine and the activity of Wnt/ß-catenin-signaling in homeostasis (normal intestine) and tumorigenesis (adenoma formation). ßcatenin is transcriptional active in the crypts in normal intestine and controls the proliferation of intestinal stem-cells (ISCS) and transient amplifying cells (TA). Upon aberrant ß-catenin activation adenomas arise due to uncontrolled proliferation (Barker et al., 2009).

For controlled homeostasis which includes the division of intestinal stem-cells and transient amplifying cells Wnt/B-catenin signaling activity is indispensable. Accordingly, ablation of TCF4, ß-catenin, or ectopic expression of the inhibitor Dickkopf 1 (DKK1) lead to loss of proliferative epithelial cells in adult and embryonic intestine (Korinek et al., 1998a;Korinek et al., 1998b;Pinto et al., 2003). In contrast, the initiation of cell cycle arrest at the crypt-villus border is of importance for the differentiation into mature epithelial, intestinal cell types. Consequently, a gradient of active Wnt-signaling is formed, with the highest activity in the bottom of the crypts accompanied with high nuclear ß-catenin, which gradually decreases to the interface of the villus. Thus, only membrane-bound ß-catenin is present in the villus resulting in the complete absence of Wnt-signaling in the tip of the villus (Figure 2, left) (Pinto et al., 2003;van de Wetering et al., 2002). In contrast, the Wnt-antagonizing bone 
morphogenic protein (BMP) pathway is active in the mesenchyme of the villi and thereby inhibits canonical Wnt-signaling in the intestinal epithelium (Figure 2, left)(Crosnier et al., 2006; Hartenstein et al., 2010; He et al., 2004b). In addition, canonical Wnt-signaling regulates the intestinal homeostasis by controlling the expression of other factors, including the ephrin ligands (ephrin) and receptors (EPH). A complex expression pattern of $\mathrm{EPH} / \mathrm{ephrin}$ determines the position of differentiated cells along the crypt-villus-axis. EPHB2/3 receptors are strongly expressed in the crypts, ephrinB1 ligands in the villi. Consequently, a gradient of receptors and ligands is formed, which creates a unidirectional migration of differentiating cells from the crypt to the villus (Batlle et al., 2002). Moreover, transforming growth factor $\beta$ (TGFß) and bone morphogenic protein (BMP) signaling in the villus inhibit Wnt/B-catenin signaling and function as growth repressors in differentiated epithelial cells (Haramis et al., 2004; Hardwick et al., 2004;Sancho et al., 2004) Thus, active and a well-defined dosage of Wnt/ß-cateninsignaling is indispensable in intestinal homeostasis.

To that effect, the majority of intestinal sporadic and hereditary adenomas derive from cells with aberrant stabilization of ß-catenin due to truncating mutations in Adenomatouspolyposis-coli (APC) (80\%) (Ichii et al., 1993;Nagase and Nakamura, 1993). Less colorectal carcinomas (CRC) occur upon stabilization of ß-catenin by mutations in Axin2 or ß-catenin itself, which abolish the phosphorylation and subsequent degradation of ß-catenin (Ilyas et al., 1997; Liu et al., 2000). Taken together, APC and ß-catenin mutations are the initial events in more than $90 \%$ of intestinal tumors (Fearon and Vogelstein, 1990). Increased ß-catenin activation leads to uncontrolled proliferation which results in a well-known sequence of histopathological changes. Dysplastic/hyperplastic crypts grow consequently out into microadenomas, which give rise to adenomas, carcinomas and subsequently invasive tumors (Fearon et al., 1990; Fodde et al., 2001)(Figure3).

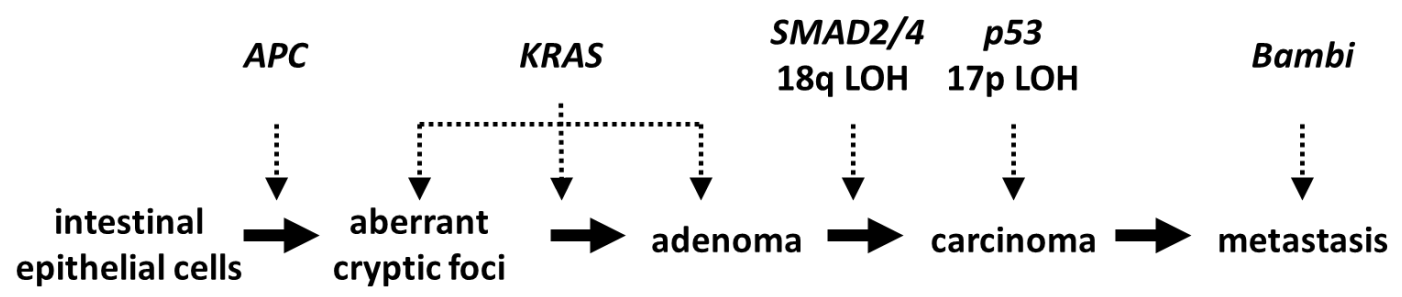

Figure 3: Gene mutations during the adenoma-carcinoma sequence Development of intestinal adenomas occur following ß-catenin stabilization due to APC truncations or mutations of $ß$-catenin itself and mutations of $K R A S$. Additional mutations resulting in loss-of-function of tumor suppressors, like SMAD2/4, p35 and Bambi induce the transformation into aggressive carcinomas. (adapted from Vogelstein and Kinzler, 1993) 
It has been shown, that deletion of APC in Leucine-rich repeat-containing G-protein coupled receptor 5 (LGR5) positive stem-cells leads to transformation of these cells to growing microadenomas that develop into macroscopic adenomas within three to five weeks (Barker et al., 2009).

However, stabilized ß-catenin is not sufficient to induce the progression of adenoma into carcinoma. Additional mutations resulting in loss-of-function of tumor suppressors (e.g. sisters and mothers against decapentaplegic (SMAD), p35 or Bambi or activation of V-Ki-ras2 Kirsten rat sarcoma viral oncogene homolog (KRAS) are necessary to drive the progression of adenomas into aggressive carcinomas (Figure3) (Fearon et al., 1990;Fodde et al., 2001;Vogelstein and Kinzler, 1993; Vogelstein and Kinzler, 2004;Wang et al., 2004).

Despite its fundamental role during canonical Wnt-signaling, ß-catenin acts as in processes of intracellular adhesion (Polakis, 2000). For cell-cell adhesion, cytosolic ß-catenin is recruited to the plasma membrane where it associates with E-cadherin. Consequently, adherence junctions are formed and stabilized which promotes a functional tissue architecture and morphogenesis (Gumbiner, 2000;Jamora and Fuchs, 2002). In tumorigenesis both functions of ß-catenin are often deregulated, which leads to aberrant ß-catenin accumulation, increases target gene expression and to loss of cell-cell adhesion (Brembeck et al., 2006). These transformations promote enhanced migration and proliferation leading to invasion and metastasis (Perl et al., 1998).

\subsection{The BCL9 proteins}

\subsubsection{BCL9 and BCL9-2 encode the vertebrate orthologue of Drosophila Legless}

In 1998 B-cell CLL/lymphoma 9 protein (BCL9) was found to be overexpressed in B-cell lymphomas caused by a $t(1 ; 14)(q 21 ; q 32)$ trans-location of the $B$-cell lymphoma gene locus (Willis et al., 1998). More than 10 years later the orthologue of BCL9, Legless (Igs), was discovered in Drosophila in a genetic screen for dominant suppressors of the classical Wntphenotype. Rescue experiments in $\operatorname{lgs}^{-\%}$ mutants revealed that the function of the segment polarity gene Igs/BCL9 is that of an absolutely required co-factor in Wnt/ß-catenin signaling in Drosophila. Maternal replacement of $\mathrm{BCL9}$ in $\operatorname{lgs}^{-\%}$ mutants was sufficient to achieve a complete rescue (Kramps et al., 2002). BCL9-2, the vertebrate orthologue of BCL9 was 
discovered as a ß-catenin related protein from a yeast-two-hybrid screen of a mouse cDNA library using a ß-catenin armadillo repeat domain as bait (Brembeck et al., 2004;Kramps et al., 2002). Seven domains of the BCL9 proteins are highly conserved with $90 \%$ amino acid sequence identity (Brembeck et al., 2004;Kramps et al., 2002)(Figure 4). Whereas vertebrate BCL9 proteins share only $35 \%$, vertebrate BCL9-2 proteins share $60 \%$ overall amino acid sequence identity (Brembeck et al., 2006).

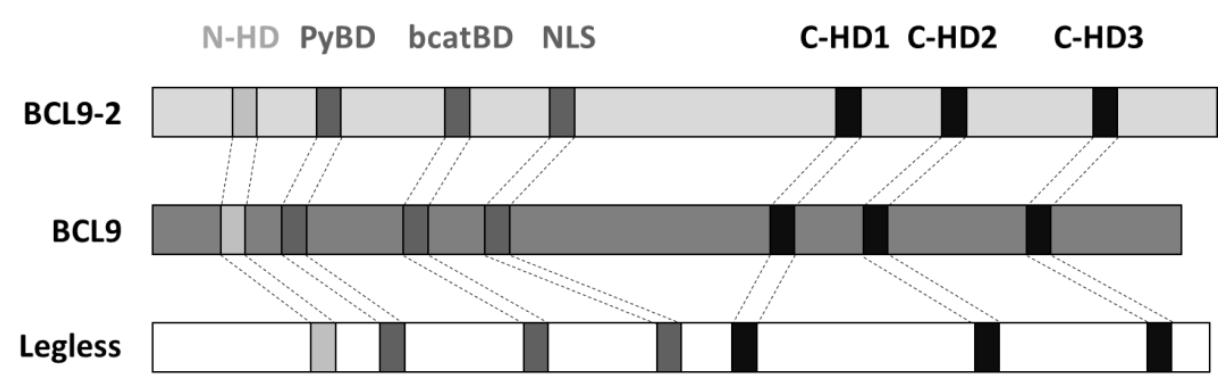

Figure 4: Schematic overview of the BCL9 proteins including seven evolutionary conserved domains The vertebrate BCL9, BCL9-2 proteins and the Drosophila homolog Legless contain seven domains, which share up to $90 \%$ amino acid sequence homology: a N-terminal homology domain (N-HD), a Pygopus binding domain (PyBD), a ß-catenin binding domain (bcatBD), a classical nuclear localization signal (NLS) and three C-terminal homology domains (C-HD1, C-HD2 and C-HD3). (Brembeck et al., 2006)

The N-terminus harbors an N-terminal homology domain (N-HD), which contains a putative sumoylation motif ( $\left.K^{*} K^{*} K X E / D\right)$ (Melchior, 2000). All BCL9 family members also harbor a ßcatenin binding domain (bcatBD), a Pygopus binding domain (PyBD), a domain containing an un-functional classical nuclear localization signal (NLS, KRRK) and three C-terminal homology domains (Figure 4). In addition, the N-HD domain of BCL9-2 contains a functional nuclear localization signal (PRSKRRC). Consequently, deletion of the N-HD of BCL9-2 and therefore the NLS leads to cytoplasmatic de-localization (Adachi et al., 2004;Brembeck et al., 2004;Hicks and Raikhel, 1995). In contrast, BCL9 and Legless are no nuclear proteins. Both require Pygopus2 to be trans-located into the nucleus (Adachi et al., 2004;Brembeck et al., 2004;Townsley et al., 2004).

\subsubsection{The BCL9/Legless proteins are co-factors of the Wnt/ß-catenin signaling pathway}

Legless and BCL9 are essential for canonical Wnt-signaling in Drosophila. They recruit Pygopus (PYGO) to the ß-catenin/TCF complex to transactivate ß-catenin-dependent target genes. The Pygopus protein family consists of the two homologs Pygo1 and Pygo2 in vertebrates which contain two highly conserved domains: an N-terminal homology domain (N-HD) interacting 
with BCL9/Legless proteins and a C-terminal PHD (plant homeo-domain) zinc finger motif (Belenkaya et al., 2002;Kramps et al., 2002) which was shown to mediate trans-activatory properties. Moreover, Pygopus contributes to the activation of gene expression by recruiting chromatin remodeling factors and by modulating Histone modifications (see 1.4.3)(Belenkaya et al., 2002;Kessler et al., 2009;Kramps et al., 2002;Nakamura et al., 2007;Stadeli and Basler, 2005).

In vertebrates Pygopus binding to BCL9-2 is not absolutely required to co-activate gene transcription of Wnt/ß-catenin target genes (Adachi et al., 2004). In addition, BCL9-2 binding promotes the trans-location of $ß$-catenin into the nucleus and therefore the expression of target genes. The switch between the adhesive and transcriptional function of ß-catenin is modulated by phosphorylation of thyrosine142 (Y142) of ß-catenin, which leads to preferred binding of the protein to BCL9-2 instead of $\alpha$-catenin (Brembeck et al., 2004).

BCL9-2 enhances Wnt/ß-catenin activity. For this function the C-terminus harboring the three C-HD is indispensable. In addition, BCL9 transcriptional co-activity requires the C-terminal region as well, although the mechanism is still unknown (Adachi et al., 2004;Sustmann et al., 2008).

\subsubsection{BCL9 proteins in development, regeneration and tumorigenesis}

Wnt/ß-catenin signaling is crucial for the dorsal-ventral mesoderm patterning in early, and organ specification in late embryonic development of Danio rerio (Huelsken and Birchmeier, 2001;Moon et al., 2002). For Wnt8/ß-catenin signaling mediated mesoderm patterning in Danio rerio BCL9-2, but not BCL9/Legless is indispensable (Brembeck et al., 2004). Moreover, other Wnt/B-catenin-dependent developmental processes, like the posteriorization of anterior neuroectoderm (Lekven et al., 2001) are BCL9-2 independent (Brembeck et al., 2004). In contrast, BCL9 and BCL9-2 are required for the activation of $\mathrm{Wnt} / \beta$-catenin signaling in adult mammalian myogenic progenitors during skeletal muscle regeneration. Under physiological conditions, Wnt1/3A/ß-catenin signaling promotes differentiation of proliferating myogenic progenitors in mice (Brack et al., 2008;Rochat et al., 2004). Reduction of BCL9/BCL9-2 abrogates myogenic differentiation (Brack et al., 2009). Thus, the BCL9 proteins function as activators of Wnt/ß-catenin signaling in a temporal and cellular context dependent manner in vivo. 
Conditional ablation of BCL9/BCL9-2 in mouse intestinal epithelium leads to decreased expression of intestinal stem-cell markers like SRY-related HMG-box gene (SOX6) and LGR5. Moreover, knockout of the BCL9 proteins impaired the regeneration capacity of ulcerated colonic epithelia. Induction of dysplastic adenomas by dimethylhydrazine and DSS led to increased $\mathrm{Wnt} / \mathrm{\beta}$-catenin signaling activity associated with nuclear $\beta$-catenin accumulation in wild-type and BCL9/BCL9-2 ablated tumors. Here a subset of the Wnt/ß-catenin target genes was reduced in knockout mice compared to wild-type tumors including Axin2, prosperorelated homeobox 1 (Prox1), Lef1 and Tcf1 (Deka et al., 2010) . Moreover, carcinomas occurred with similar incidence, although the size of mutant tumors was generally smaller. Comparative transcriptional expression profiles of wild-type and mutant carcinomas further revealed a role of $B C L 9 / B C L 9-2$ in the control of epithelial-mesenchymal-transition (EMT) indicated by reduction of fifteen EMT-marker genes coding for proteins like Slug (Snail2), Snail (Snail1), Vimentin (Vim), Zeb1 and Zeb2. Moreover, fifty stem-cell related genes like Lgr5 and Sox6 were decreased in the gene set of BCL9/BCL9-2 conditional knockout mice indicating a role of the BCL9 proteins in modulating stem-cell-like traits in colon cancers (Deka et al., 2010). Thus, Deka et al. pointed out that the function of BCL9/BCL9-2 function in intestinal regeneration and further, in colonic tumorigenesis by controlling a subset of Wnt/ß-catenin target genes that are implicated in EMT and stem-cell traits. However, the mouse model used by Deka et al. did not provide any information about the particular role of each of the BCL9 proteins. Thus, the observed effects on regeneration and tumorigenesis could have been mediated by BCL9, BCL9-2 or both. In contrast, Matsuura et al. investigated the particular function of BCL9-2. Complete knockout of BCL9-2 lead to growth arrest due to placental defect at around E10 resulting in embryonic lethality at E10.5 to E11.0, whereas heterozygous $\mathrm{BCL}^{+/-}$mice were healthy and fertile. Those placental phenotypes are reminiscent of those resulting from cell fusion pathway defects (Matsuura et al., 2011). Moreover, intestinal stem-cells with high Wnt/ß-catenin signaling are fusion partners of transplanted bone marrow-derived cells in tissue regeneration in the intestine. Transformed intestinal cells of $\mathrm{APC}^{\mathrm{MIN} /+}$ mice exhibit an increased frequency of fusion with bone marrow-derived cells (Lu and Kang, 2009).

The BCL9 proteins were described to play a role during tumorigenesis in different tumor entities. BCL9 and BCL9-2 overexpression was found in colorectal cancers tumors (Adachi et al., 2004;Brembeck et al., 2011;de la Roche et al., 2008). Also, BCL9-2 mRNA expression correlated with the progressive grades of colorectal neoplasias (Sakamoto et al., 2007). In this context BCL9 proteins induce canonical Wnt-signaling and thereby increase the ß-catenin mediated tumorigenic potential of tumor cells by contributing to stem-cell straits and EMT 
(Matsuura et al., 2011). Disruption of the BCL9 and B-catenin interaction leads to decreased oncogenic Wnt-signaling activity and is therefore believed to be a promising therapeutic approach for treating Wnt-dependent cancers (de la Roche et al., 2012a;Takada et al., 2012).

BCL9-2 overexpression was found in breast cancers (Zatula and Brembeck, unpublished)(Mani et al., 2009;Toya et al., 2007). Human breast cancer tissue arrays revealed a correlation between high BCL9-2 and the expression of the hormone receptors of Estrogen (ER), Progesterone (PR), the human epidermal growth factor receptor 2 (HER2) (Zatula and Brembeck, unpublished) and of the proto-oncogene c-myc and p53 (Toya et al., 2007). Moreover, high BCL9-2 expression was found during the ß-catenin/Wnt-dependent ductal outgrowth and during pregnancy in mouse mammary glands (Boras-Granic and Wysolmerski, 2008) suggesting the contribution of BCL9-2 to ß-catenin/Wnt activity dependent proliferation during these stages of development (Zatula and Brembeck, unpublished).

Investigations regarding the BCL9 proteins mainly focused on their ß-catenin associated function as activators of canonical Wnt-signaling (Adachi et al., 2004; de la Roche et al., 2008;Kramps et al., 2002;Sakamoto et al., 2007;Sustmann et al., 2008). BCL9-2 plays an important role in $\mathrm{Wnt} / \beta$-catenin pathway-mediated cell fusion, during embryonic development, tissue regeneration and cancer development but appears to be dispensable in intestinal homeostasis (Adachi et al., 2004;Brembeck et al., 2011;de la Roche et al., 2008;Deka et al., 2010). The gene locus of BCL9 was shown to be trans-located in B-cell lymphomas leading to aberrant BCL9 protein expression, but nothing is known about the mechanisms which lead to overexpression of BCL9-2 in cancers so far. However, the particular function of BCL9 and BCL9-2 in tumorigenesis is not well understood. Until now, only the impact of the BCL9 proteins regarding their Wnt/ß-catenin-dependent function had been investigated in detail (Adachi et al., 2004;Brack et al., 2009;Brembeck et al., 2006;Brembeck et al., 2004;Kramps et al., 2002). Moreover, a mechanism which explains the molecular ß-catenindependent or/and independent capacity of BCL9 and BCL9-2 is still to be found.

\section{3. $\mathrm{K} 19$ dependent $\mathrm{BCL9-2}$ overexpression in vivo}

Since BCL9-2 is overexpressed in many human and mouse tumors we established a BCL9-2 overexpression mouse model to further investigate the function of this protein (Brembeck et al., 2011)(Zatula and Brembeck, unpublished). In our mouse model ectopic expression of flagtagged BCL9-2 was induced by the promoter of the epithelial specific Keratin 19 gene (K19)(for 
detailed information see Materials and Methods 3.9.1). Flag-tagged BCL9-2 is consequently expressed in all simple epithelia including the stomach, intestine, liver, kidney, pancreas and mammary glands (Brembeck et al., 2001;Brembeck and Rustgi, 2000).

Aged transgenic mice (more than 15 months of age) developed macroscopic tumors in the pancreas, the intestine and in mammary glands. In contrast to undifferentiated pancreatic and intestinal tumors, mammary gland tumors were well differentiated and composed of ductallike and myoepithelial-like structures. Interestingly, mammary gland tumors were positive for nuclear Estrogen- (ER $\alpha$ ) and Progesterone- (PR) receptors. Accordingly, primary cell cultures of K19-BCL9-2 tumors were dependent on Estrogen. Knockdown of BCL9-2 in MCF7 breast cancer cells lines led to a transient reduction of ER $\alpha(E s r 1)$ and ER $\alpha$ target gene expression, indicating a regulatory role of BCL9-2 in Estrogen receptor signaling. Interestingly, MCF7 cells contain no transcriptionally active ß-catenin although BCL9-2 is highly expressed. This suggests a novel ßcatenin-independent mechanism (Zatula and Brembeck, unpublished).

\subsection{Transcriptional regulatory mechanisms}

The complex regulation of gene expression is accomplished by the collective activity of diverse transcriptional regulatory proteins. Transcription factors (TF) modulate the action of RNA polymerases through binding to specific cis-regulatory sequences within the promoter of a gene (Matsui et al., 1980;Segall et al., 1980).

\subsubsection{Basal transcriptional regulatory mechanisms}

\subsubsection{Basal transcriptional activation}

To initiate gene transcription RNA polymerase II requires the binding of specific transcription factors to attach to core promoter regions. The pre-initiation complex (PIC), consisting of general transcription factors (GTFs), transcription factor II D (TFIID) and polymerase II subsequently initiates transcription supported by variable co-factors (for example, see 1.4.2 and 1.4.3)(Blackwood and Kadonaga, 1998). Commonly, eukaryotic genes such as CDX1 harbor a transcription initiation site located in a region of approximately -30bp from the transcription start (TS) site, called canonical TATA box that binds the pre-initiation complex PIC trough transcription factor II D (Burley and Roeder, 1996;Nikolov et al., 1996;Suzuki et al., 2001). 
However, approximately $76 \%$ of all human promoters transcribed by RNA polymerase lack TATA-like elements (TATA-less promoters) and even initiator elements (Weis and Reinberg, 1992). Initiator elements, downstream core promoter elements (DPE) and TBIID recognition element (BRE) comprising the transcription start site and are able to facilitate the binding of TBP in TATA-less promoters with or without the participation of additional cis-acting elements (Bucher, 1990;Javahery et al., 1994;Smale and Baltimore, 1989) (Figure 5). Among others, motifs corresponding to specificity protein 1 (SP1) binding sites can be present instead of TATA or initiator elements and are therefore enriched in TATA-less promoters.

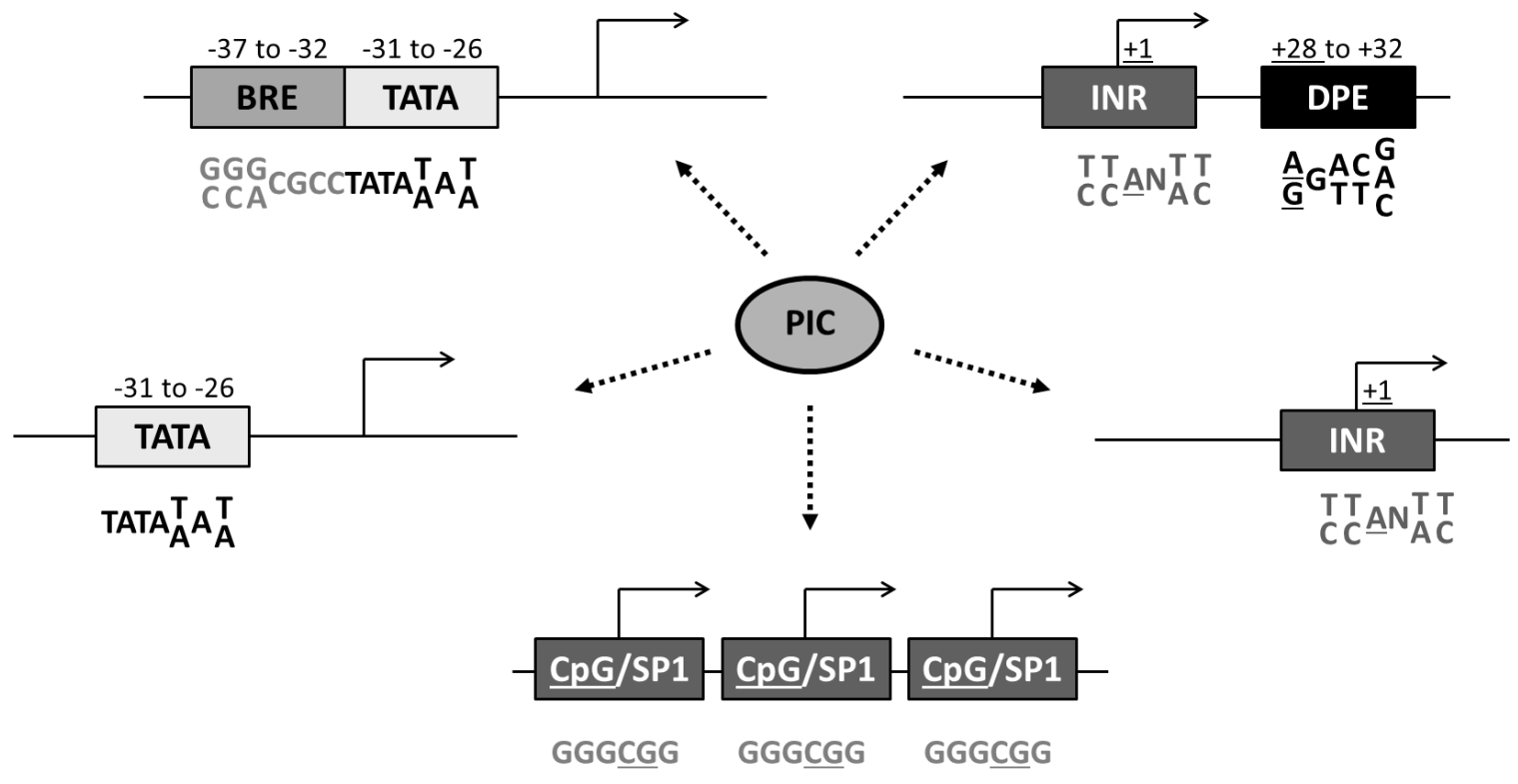

Figure 5: Transcriptional enhancers cooperating with the pre-initiation complex and initiating gene transcription through a variety of core promoters

The pre-initiation complex (PIC) and additional transcriptional enhancers participate in RNA polymerase II mediated transcription by binding to core promoter elements. Each of the regulatory elements is present in a subset of core promoters in different distributions, as indicated. (adapted from Blackwood et al., 1998)

\subsubsection{Transcriptional activation through Specific protein (SP) transcription factors in normal homeostasis and tumorigenesis}

SP proteins belong to the family of SP/KLF transcription factors and can either enhance or repress the transcription of target genes. The family of SP/KLF factors includes the SP proteins (SP1 to SP9) and the krüppel-like factors (KLF1 to KLF16) (Simmen et al., 2010; Black et al., 2001; Suske, 1999). The different family members have been shown to preferentially, but not exclusively bind to different sequence motifs. Accordingly, KLFs prefer CACCC-boxes (Shields and Yang, 1998), whereas SPs preferentially bind to GC-boxes (Suske, 1999). SP motifs are frequently present in CpG islands (see 4.2.1) which are often un-methylated, GC-rich 
sequences (Adachi and Lieber, 2002). CpG islands are commonly overrepresented in cis transcription factors (Brandeis et al., 1994). Moreover, binding of SP1 to GC-rich elements can activate gene transcription without or in combination with initiator elements (INR) in absence of any TATA or downstream core promoter elements (Smale et al., 1989;Smale et al., 1990) (Figure 5).

SP1 was one of the first identified basal mammalian transcription factor (Kadonaga et al., 1987). SP1-knockout mice are early embryonic lethal at E10.5. In contrast, knockout of its closest relative, SP3, causes postnatal death (Kruger et al., 2007). SP1 and SP3 share over $90 \%$ DNA sequence homology in their DNA binding domain which consists of three adjacent $\mathrm{Cys}_{2} \mathrm{His}_{2}$-type zinc fingers (Suske et al., 2005). SP1 and SP3 have different isoforms, which are generated through alternative splicing of SP1 and alternative translational initiation sites in SP3 (Li et al., 2004;Sapetschnig et al., 2004). SP1 and the longest SP3 variant contain two transactivation domains ( $A D 1$ and $A D 2$ ) and a domain, which is used for synergistic activation (SAD). In contrast to SP1, SP3 contains an additionally inhibitory domain (ID) (Figure 6). In addition, both transcription factors are post-translationally modified. SP1/3 can be sumoylated - which represses SP1 activity - or acetylated and phosphorylated, which results in increased transcriptional activity (Li and Davie, 2010; Li et al., 2004).

SP1

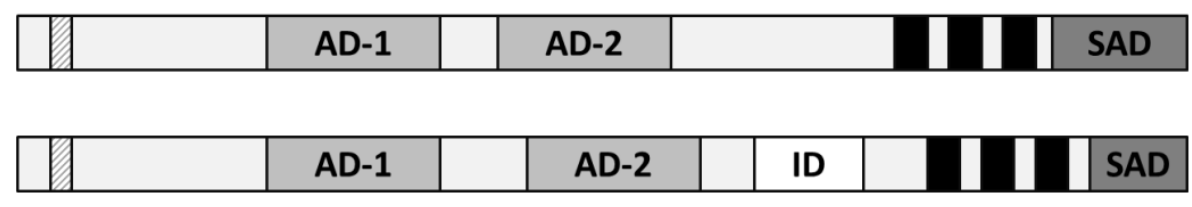

SP-box Zink-finger

Figure 6: Schematic overview about the protein structure and modification motifs of SP1 and SP3 SP1 and SP3 contain an SP-box, a highly conserved DNA-binding domain consisting of three Zink-fingers two trans-activation domains (AD) and a C-terminal synergistic activation-domain (S-AD). In addition, SP1 contains an inhibitory-domain. (adapted from Bouwman et al., 2002)

SP1 and SP3 can either induce or inhibit gene transcription, in dependency on their protein variant and post-translational modification. To this end, SPs directly interact with proteins in the pre-initiation complex and other nuclear cofactors which comprise the basal transcription factors and consequently initiate transcription. SP1-binding sites are commonly present in the promoters of TATA-less house-keeping genes such as acetyl-CoA synthetase 1 (Ikeda et al., 2001; Lin et al., 1996;Zhu et al., 2008). SP1 and SP3 target gene products are involved in processes such as differentiation and cell cycle progression (Davie et al., 2008). Moreover, overexpression of SP1 proteins in colon-, breast-, pancreatic- and gastric- and lung -cancers (Li et al., 2010) results in increased expression of tumorigenesis-related genes. SP1 was shown to 
interact with several transcription factors such as SMAD2/3/4 (Feng et al., 2000), wild-type or mutated p53 (Schavinsky-Khrapunsky et al., 2003) and OCT1 (Strom et al., 1996), respectively. For example, in breast cancer cells SP3 plays a role for the ER $\alpha$ dependent trans-activation of the promoter of the vascular endothelial growth factor receptor 2 (VEGFR2) (Higgins et al., 2006a;Higgins et al., 2006b). In addition, transcription of VEGF is regulated by SP1 in pancreatic tumors (Safe and Abdelrahim, 2005). Moreover, it has been shown that mithramycinA mediated inhibition of SP1 expression is correlated with anti-angiogenic effects in human pancreatic cancers, suggesting that SP1 might be a useful therapeutic target (Jia et al., 2007; Yuan et al., 2007).

\subsubsection{Mechanisms of ß-catenin dependent transcriptional regulation of Wnt/ß- catenin target genes}

Upon Wnt-stimulation, ß-catenin trans-locates to the nucleus (see 1.1.1) and binds to LEF/TCF transcription factors (Behrens et al., 1996). LEF/TCF transcription factors require specific consensus sequences in the promoters of target genes, also known as Wnt responsive elements $[(a / t)(a / t) C A A(a / t) G]$ (Roose and Clevers, 1999). The LEF/TCF transcription factor family consists of LEF1, TCF1 (TCF7), TCF3 (TCF7L1) and TCF4 (TCF7L2). Of note, phenotypic rescue experiments revealed that the function of LEF/TCFs is not redundant (Mao and Byers, 2011). Moreover, also opposite effects of the transcription factors had been determined; TCF3 mainly, but not exclusively, acts as a repressor, while LEF1 primary exerts transcriptional activatory functions (Yi et al., 2011). In addition, LEF/TCFs are expressed in diverse isoforms. Dependent on the experimental system and isoform which is used, TCF1 and TCF4 exert dual functions (Mao et al., 2011).

Binding of ß-catenin displaces repressors like Groucho/TLE which subsequently promotes the interaction of ß-catenin with the TATA-binding protein (TBP) and the basal transcription machinery (see section 1.4.1.1)(Bauer et al., 1998;Daniels et al., 2005). The activation of ßcatenin-dependent gene transcription additionally requires the de-condensation of chromatin (Figure 7). Therefore, chromatin remodeling factors have been identified which bind to Bcatenin (Novak and Dedhar, 1999), such as the histone acteylase CBP/p300 (CREB-binding protein)(Greaves et al., 1999), histone methyltransferase MLL2 (mixed-lineage-leukemia 2)(Willert and Jones, 2006) and BRG-1 (brahma related gene 1) which is a component of a nucleosome remodeling complex (Barker et al., 2001). In addition, ß-catenin binds to DNA helicasess such as Pontin52 (Pon) via its armadillo repeats (Bauer et al., 1998), which further 
supports the transcriptional activation of ß-catenin target genes (Figure 7). Co-factors, such as BCL9 and Pygopus, additionally promote the transcriptional activity (see 1.4.4).

Without Wnt-stimulation, ß-catenin is degraded and the expression of target genes is transcriptionally inhibited by different mechanisms (Figure 7). TCF transcription factors reside in the nucleus, bound to the promoters of target genes. Thus, in the absence of ß-catenin, TCFs act as transcriptional inhibitors (Gregorieff and Clevers, 2005; Gregorieff et al., 2004). Transcriptionally inactive TCFs bind to repressive nuclear factors such as Groucho/TLE (Cavallo et al., 1998;Levanon et al., 1998), which then recruit histone deacetylases such as HDAC1 (Chen et al., 1999). De-acetylation of histones subsequently results in condensation of the chromatin which represses transcription.

\section{A. Transcription of target genes}

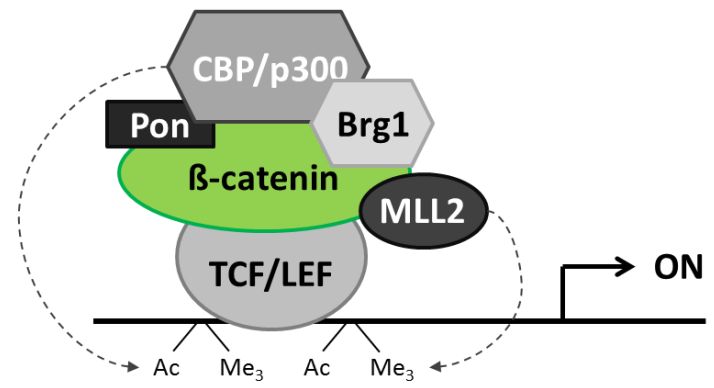

\section{B. Repression of target genes}

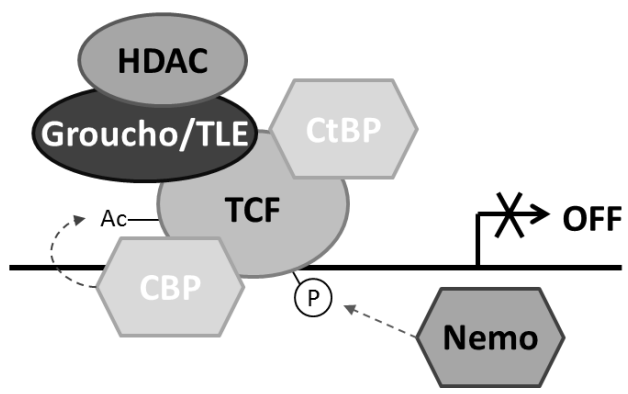

Figure 7: Transcriptional regulation of Wnt/B-catenin target genes

(A) Transcriptionally active $B$-catenin binds to LEF/TCF and recruits the helicase Pontin52 (Pon) and different chromatin remodeling factors like BRG-1 or CBP and MLL2 (MLL) which acetylate (AC) and methylate $\left(\mathrm{Me}_{3}\right)$ histones, respectively. (B) TCF represses gene transcription in the absence of $\mathrm{B}$-catenin through the interaction with different co-repressors such as Groucho/TLE and CtBP which recruit chromatin remodeling factors like histone deacetylases (HDAC). Additionally, TCF is modified by CBP acetylases and Nemo phosphatases which inhibit binding to the DNA and B-catenin, respectively. (adapted from Parker et al., 2007)

Binding of CtBP to TCF leads to epigenetic chromatin modifications including histones demethylation through the interaction with additional co-repressors (C-terminal binding protein) (Arce et al., 2006;Brannon et al., 1999;Chinnadurai, 2002). In addition, modifications of TCF promote the repression of target genes. NLK/Nemo kinases inhibit the DNA-binding affinity of the transcription factor through phosphorylation of TCF (Ishitani et al., 2003). Moreover, CBP interacts with the TCF-DNA-binding domain (HMG-box) and acetylates TCF at its N-terminal ßcatenin binding domain which prevents binding to ß-catenin and consequently the activation of transcription (Figure 7)(Waltzer and Bienz, 1998). 


\subsubsection{Crosstalk of LEF/TCF and SP1 in the transcriptional control of target gene expression}

A crosstalk between LEF/TCF transcription factors and the function of SP transcription factors has been described (Clements et al., 1996;Rossi et al., 2006;Sheridan et al., 1995;Thorpe et al., 2005; Weidinger et al., 2005;Yamaguchi et al., 1999).

During central nervous system development Wnt/ß-catenin signaling activates the expression of SP5, which appeared to repress the transcriptional activation of SP1 target genes (Fujimura et al., 2007).

Moreover, SP5 and the homologue SP5like mediate the function of Wnt8 in neuroectoderm and mesoderm patterning during zebrafish gastrulation (Thorpe et al., 2005; Weidinger et al., 2005). In mouse primitive steak and tailbud development Wnt responsive elements act in cooperation with SP1 binding sites in the promoter of T (Brachyury) to activate the expression of this gene (Clements et al., 1996;Yamaguchi et al., 1999). In addition, in vitro analyses suggest that LEF1 dependent transcription of the HIV-1 promoter requires the participation of SP1 in Jurkat T cells (Sheridan et al., 1995). However, in human astrocytic cells TCF4 decreases the SP1 mediated transcription of the HIV-1 promoter through the prevention of SP1-DNA association. In this regard, interaction of TCF4 and SP1 leads to de-phosphorylation of SP1, which results in inhibited transcriptional activity (Rossi et al., 2006).

The interaction of SP1 and LEF/TCF transcription factors and their modulation of each other's transcriptional functions had been described in many different systems (Clements et al., 1996;Pesce et al., 1999;Rossi et al., 2006;Sheridan et al., 1995;Thorpe et al., 2005;Weidinger et al., 2005;Yamaguchi et al., 1999). The influence of SP1 on the function of TCF and LEF transcription factors differs in dependence of the genetic background. However, the authors pointed, that the specific functions of these interactions are highly context dependent (Rossi et al., 2006; Sheridan et al., 1995). Thus, the consequence of an interaction of SP and LEF/TCF could differ in dependence of the molecular background. 


\subsubsection{Transcriptional regulatory mechanism of Wnt/ß-catenin target genes by the BCL9 and Pygopus co-factors}

In addition to basal transcriptional regulators and chromatin remodeling factors, the transcription of Wnt/B-catenin target genes requires the co-activation by BCL9 and Pygopus proteins.

Nuclear ß-catenin, bound to LEF/TCF, interacts with BCL9/BC9-2, which further recruits Pygopus1/2 proteins (Figure 8)(Belenkaya et al., 2002;Kramps et al., 2002). Studies in Drosophila suggested that BCL9/Legless functions as an adaptor protein which mediates the recruitment of Pygopus to the basal transcriptional machinery. However, many studies demonstrated, that BCL9 and BCL9-2 themselves act as transcriptional co-activators in vertebrates (Adachi et al., 2004;Brembeck et al., 2011;Deka et al., 2010;Sustmann et al., 2008). In this regard, the C-terminus of the BCL9 proteins was shown to be essential for their coactivatory function, although the underlying mechanism is still unknown (Adachi et al., 2004;Sustmann et al., 2008). In contrast, Pygopus mediated transcriptional activity has been studied in more detail:

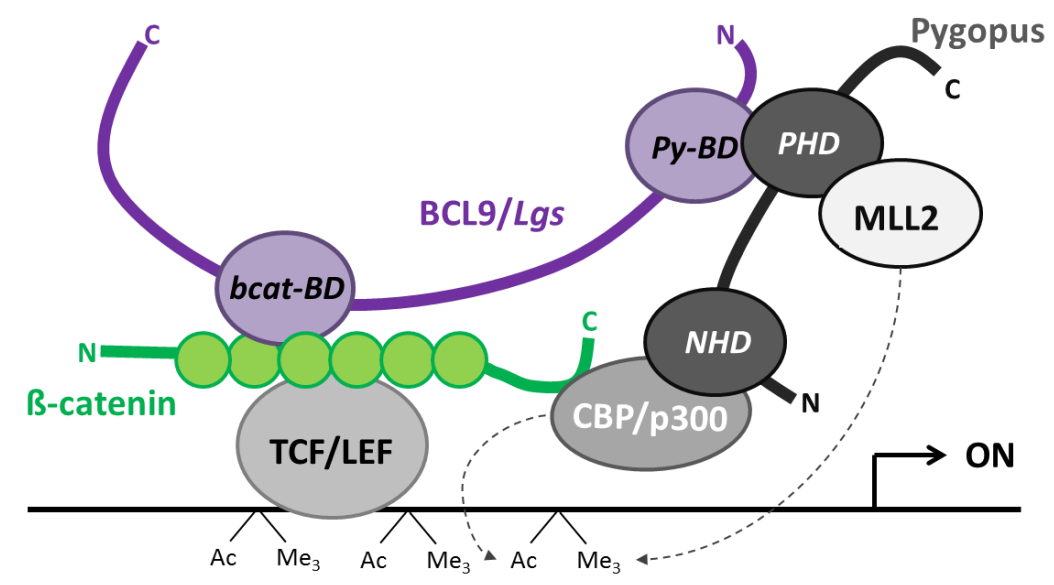

Figure 8: BCL9/Pygopus dependent transcriptional activation of Wnt/ß-catenin target genes

LEF/TCF transcription factors bind to ß-catenin, which is recruited by BCL9/Legless through its ß-catenin binding domain (bcat-BD). The Pygopus binding domain (Py-BD) of BCL9/Legless recruits Pygopus which is bound to the MLL2 methyltransferase through its PHD domain (PHD). Pygopus' NHD domain binds to $\mathrm{CBP} / \mathrm{p} 300$ acetyltransferase which additionally interferes with ß-catenin which consequently activates gene transcription. (adapted from Andrews et al., 2009)

Pygopus proteins modulate transcription through their C-terminal PHD plant homeo-domain (PHD) zinc finger motif and through their $\mathrm{N}$-terminal homology domain (NHD). The PHD interferes with MML2 histone methyltransferases (HMT) which promotes transcription through trimethylation of histone 3 lysine residues $\left(\mathrm{H}_{3} \mathrm{~K}_{4} \mathrm{Me}_{3}\right)$ (Chen et al., 2010; Gu et al., 2009). In addition, Pygopus 2 had been shown to bind to the histone acetyltransferase 
CBP/p300 (CREB binding protein). This interaction might promote the acetylation of histone 3 which subsequently results in de-condensation of chromatin and therefore transcriptional activation of Wnt/B-catenin target genes (Figure 8)(Andrews et al., 2009).

Interestingly, previous studies from our group indicated, that not all BCL9-2 dependent Wnt/ßcatenin target genes require the participation of Pygopus proteins. Consequently, BCL9-2 possibly co-activates the expression of Wnt/ß-catenin target genes independently of Pygopus interaction. 


\section{Aim of this work}

The role of BCL9/Legless and Pygopus in Drosophila has been well described. However, little is known about the role of the BCL9 and Pygopus proteins in vertebrates. Our group has previously identified BCL9-2, the orthologue of BCL9/Legless, which also acts as a co-activator in the canonical Wnt/ß-catenin pathway. This study was carried out in regard to the function of BCL9 and Pygopus proteins 2 proteins during intestinal carcinogenesis. This work presented here will focus on the function of the BCL9 proteins

The aim of this study was to investigate the role of BCL9 and Pygopus proteins in intestinal homeostasis and during intestinal carcinogenesis. De-regulation of BCL9-2 in tumors might be further associated with de-regulation of other genes, which may contribute to tumor development and progression. We hypothesize that BCL9-2 in vertebrates acts not as a general enhancer of Wnt/B-catenin-dependent transcription, but rather regulates a specific subset of target genes, possibly also independent of Wnt/ß-catenin signaling by a ß-catenin independent mechanism.

To address the role of BCL9 proteins during the multistep-model of intestinal tumorigenesis, the expression of BCL9/BCL9-2 was examined in normal, intestinal epithelia and during different stages of carcinogenesis using immunohistochemistry. In addition, adenoma formation of K19-BCL9-2/APC ${ }^{\mathrm{MIN} /+}$ compound mutant mice was followed to investigate the influence of BCL9-2 overexpression on tumor development.

We further investigated the specific gene signature that is induced by the BCL9 and BCL9-2 Coactivators in comparison to known Wnt/ß-catenin target genes. To this end, qPCR, Luciferase reporter gene assays and microarray analyses were performed following knock down of the proteins.

In addition, this study addressed the regulatory mechanism concerning BCL9-2 mediated transcriptional regulation. A model system was used to dissect the ß-catenin-dependent and independent functions of BCL9-2. For this purpose, two Luciferase-reporters containing the proximal promoter of a BCL9-2/ß-catenin-dependent- and a ß-catenin independent- BCL9-2 target gene were studied in detail. Finally, transcription factor binding elements which were present in both promoters were mutated. The activity of mutated Luciferase-reporters was analyzed in colon cancer cell lines following knockdown of BCL9-2. To identify a transcription factor that acts as putative BCL9-2 interaction partner from mediating a ß-cateninindependent co-activatory function of BCL9-2 co-immunoprecipitations were performed. 


\section{Materials and Methods}

Unless stated otherwise, standard protocols were followed (Michael R.Green and Joseph Sambrook, 2012). Likewise, standard buffers and solutions were prepared and used as described in Sambrook, 2012.

\subsection{General materials and chemicals}

Disposables and basic material (Table 1), instruments and equipment (Table 2), chemicals and reagents (Table 3), commercial kits (Table 4), enzymes (Table 5) as well as antibodies (Table 6, Table 7 and Table 8) were obtained from the manufactures listed below.

Table 1: Disposables and basic material

\begin{tabular}{|c|c|}
\hline Materials & manufacturer \\
\hline Cryovials & Nunc \\
\hline Culture plates (50 mm and $100 \mathrm{~mm} \varnothing$ ) & Nunc \\
\hline Cuvettes & Roth \\
\hline Filters for solutions $(0.2 \mu \mathrm{m}$ and $0.45 \mu \mathrm{m})$ & Sartorius \\
\hline Flasks for cell culture $\left(75 \mathrm{~cm}^{2}\right.$ and $\left.175 \mathrm{~cm}^{2}\right)$ & Sarstedt \\
\hline Gloves (nitrile, latex) & Sempermed \\
\hline Hybond-P PVDF membrane & GE Healthcare \\
\hline Hypodermic needle (23 G) & Braun \\
\hline Parafilm & Pechiney Plastic Packaging \\
\hline Pasteur pipettes & Peske OHG \\
\hline Petri dishes & Falcon \\
\hline Pipette filter tips (10, 200 and $1000 \mu \mathrm{l})$ & Biozym \\
\hline Pipette tips $(10,200$ and $1000 \mu l)$ & $\mathrm{MbP}$ \\
\hline Pipettes $(2,5,10$ and $25 \mathrm{ml})$ & Eppendorf \\
\hline Plates for cell culture (6-well, 24-well and 96-well) & TPP, Nunc \\
\hline
\end{tabular}




\begin{tabular}{|c|c|}
\hline Scalpels & Technic cut \\
\hline Tubes for cell culture (polypropylene, $15 \mathrm{ml}$ and $50 \mathrm{ml}$ ) & Falcon \\
\hline Tubes for cell culture (polystyrene, 15 and $50 \mathrm{ml}$ ) & Falcon, Sarstedt \\
\hline Tubes for molecular biology, Safelock (1.5 ml and $2 \mathrm{ml})$ & Eppendorf, Sarstedt \\
\hline Whatman paper & Whatman \\
\hline freezing boxes for cell cultures & Nalgene \\
\hline
\end{tabular}

Table 2: Instruments and equipment

\begin{tabular}{|c|c|}
\hline Instrument & manufacturer \\
\hline Camera DC 300 FX/Camera DFC 290 & Leica \\
\hline Cell counting chamber Neubauer & Brand \\
\hline Cell culture incubator BBD 6220 & Heraeus \\
\hline Cell culture sterile bench LaminAir HB 2448 & Heraeus \\
\hline Refrigerated Microcentrifuge & Eppendorf \\
\hline Microcentrifuge & Eppendorf \\
\hline Refrigerated Bench-Top Hood Centrifuge & Eppendorf \\
\hline Microcentrifuge MCF 2360 & MS Co. LTD \\
\hline Controlled-freezing box & Nalgene \\
\hline Electrophoresis chambers for agarose gels & Peqlab \\
\hline Electrophoresis chambers for SDS-PAGE & BioRAD \\
\hline ELISA Reader SUNRISE A-5082 & TECAN \\
\hline $\begin{array}{l}\text { Freezer }\left(-150^{\circ} \mathrm{C}\right) \text { Ultra low temperature freezer MDF- } \\
\text { C2156VAN }\end{array}$ & Sanyo \\
\hline Freezer $\left(-20^{\circ} \mathrm{C}\right)$ PremiumNoFrost & Liebherr \\
\hline Freezer $\left(-80^{\circ} \mathrm{C}\right)$ Ultra low temperature freezer U725 & New Brunswick Scientific GmbH \\
\hline Fridge $\left(+4^{\circ} \mathrm{C}\right)$ Electrolux SANTO & AEG \\
\hline Gel documentation BioDocAnalyze & Biometra \\
\hline Heating block - Thermostat plus & Eppendorf \\
\hline
\end{tabular}




\begin{tabular}{|c|c|}
\hline Ice machine ZBE 70-35 & Ziegra \\
\hline Incubator & Memmert \\
\hline Multimode Reader TriStar LB 946 & Berthold \\
\hline Micropipettes $(2,10,100,200,1000 \mu l)$ & Eppendorf \\
\hline Microscope DM 500 & Leica \\
\hline Microscope inverted DM IRB & Leica \\
\hline Microwave oven & Powerwave \\
\hline PCR cycler T3 Thermocycler & Biometra \\
\hline Pipetting assistant MATRIX & Thermo Scientific \\
\hline Power supplier EV231 & Peqlab \\
\hline Printer & Mitsubishi \\
\hline Pump VDE0530 & Adam.Baumüller GmbH \\
\hline 7900HT Fast Real-Time PCR System & Applied Biosystems \\
\hline Rotator & GLW \\
\hline IKA- Shaker MTS4 & W.Krannich GmbH+Co.KG \\
\hline Sonifier & Dr. Hielscher $\mathrm{GmbH}$ \\
\hline Spectrophotometer ND-1000 & NanoDrop \\
\hline Stereomicroscope MZ FL III & Leica \\
\hline Transilluminator UV Star & Biometra \\
\hline UV lamp EBQ100 isolated & Leica \\
\hline IKA ${ }^{\circledR}$ Vortex & IKA \\
\hline Water bath GFL 1003 & W.Krannich GmbH+Co.KG \\
\hline Water purification system & Millipore \\
\hline Western Blot Documentation LAS-4000 & Fujifilm \\
\hline Wet Transfer Apparatus & Biorad \\
\hline Shaker for bacteria & Sartorius \\
\hline NanoDrop-Spektrophotometer & Thermo Scientific \\
\hline
\end{tabular}


Inkubator BBD 6220

Rotator RM5
Thermo Scientific

Assistant 348

Table 3: Chemicals and reagents

chemical/reagent

manufacturer

\begin{tabular}{|c|c|}
\hline Acetic acid & Roth \\
\hline Agarose & Invitrogen \\
\hline APS (Ammonium persulfate) & Roth \\
\hline benzyl-coelenterazine & SYNCHEM \\
\hline Bradford reagent & BioRAD \\
\hline BrdU (Bromdesoxyuridin) & Roche \\
\hline Bromphenol Blue & Roth \\
\hline Chloroform & Roth \\
\hline Collagen & Sigma \\
\hline Collagenase/Hyaluronidase Cocktail & Stemcell \\
\hline$D(+)$-trehalose dihydrat & Roth \\
\hline DAPI (4',6-diamidino-2-phenylindole) & Sigma \\
\hline DEPC (diethyl pyrocarbonate) & Roth \\
\hline Dexamethasone & Sigma \\
\hline Dispase & Sigma \\
\hline D-Luciferin potassium salt, $99 \%$ & Synchem \\
\hline DMEM GlutaMAX'TM & Invitrogen \\
\hline DMSO (dimethyl sulfoxide) & Roth \\
\hline DTT (DL-Dithiothreitol) & Sigma \\
\hline EDTA (ethylenediaminetetraacetate) & Roth \\
\hline EGTA (ethylene glycol tetraacetic acid) & Roth \\
\hline EtBr (ethidium bromide) & Roth \\
\hline
\end{tabular}




\begin{tabular}{|c|c|}
\hline Ethanol & Chemie Vertrieb Hannover \\
\hline FBS (Fetal bovine serum) & Invitrogen, Sigma \\
\hline GeneRuler $^{\mathrm{TM}} 1 \mathrm{~kb}$ DNA Ladder & Fermentas \\
\hline Glycerol & Sigma \\
\hline Glycine & Roth \\
\hline $\mathrm{H}_{2} \mathrm{O}_{2}$ (Peroxygen) & Roth \\
\hline $\mathrm{HCl}$ (Hydrochloric acid) & Roth \\
\hline HEPES & Roth \\
\hline Immu-Mount & ThermoScientific \\
\hline Isopropanol & J.T.Backer \\
\hline $\mathrm{KAIS}_{2} \mathrm{O}_{2} \bullet 12 \mathrm{H}_{2} \mathrm{O}$ (Potassium aluminium sulfate) & Sigma \\
\hline $\mathrm{KCl}$ (potassium chloride) & Sigma \\
\hline L-glutamine & Invitrogen \\
\hline Lipofectamin 2000 & Invitrogen \\
\hline Luminol & Sigma \\
\hline Methanol & J.T.Backer, Merck \\
\hline $\mathrm{MgCl}_{2}$ (Magnesium chloride) & Roche \\
\hline $\mathrm{MgSO}_{4}$ (Magnesium sulfate) & Roth \\
\hline $\mathrm{Na}_{2} \mathrm{SO}_{4}$ (Sodium sulfate) & Roth \\
\hline Na4PPi (Sodium pyrophosphate) & Roth \\
\hline $\mathrm{NaCl}$ (Sodium chloride) & Roth \\
\hline $\mathrm{NaHCO}_{3}$ (Sodium hydrogen carbonate) & Merck \\
\hline $\mathrm{NaOH}$ (Sodium hydroxide) & Sigma \\
\hline Nicotinamid & Sigma \\
\hline Nonidet-P40 (NP40) & Sigma \\
\hline Opti-MEM $^{\circledR}$ & Invitrogen \\
\hline PageRuler ${ }^{\mathrm{TM}}$ Prestained Protein Ladder & Invitrogen \\
\hline
\end{tabular}




\begin{tabular}{|c|c|}
\hline p-Coumaric acid & Sigma \\
\hline Penicillin / streptomycin & Invitrogen \\
\hline PFA (Paraformaldehyde) & Merck \\
\hline phenyl-benzothiazole & City Chemicals \\
\hline PMSF (Phenylmethanesulfonylfluoride) & Sigma \\
\hline Polyacrylamide (30\% Acrylamide / Bis) & Roth \\
\hline Powdered milk & Roth \\
\hline Protease Inhibitor cocktail tablets, EDTA free & Roche \\
\hline restriction buffer $\mathrm{O}$ & Fermentas \\
\hline restriction buffer $\mathrm{R}$ & Fermentas \\
\hline restriction buffer $X X X$ & Fermentas \\
\hline RNA sample buffer & Fermentas \\
\hline RNase Inhibitor & Fermentas \\
\hline Roti $^{\circledR}$-Histokitt & Roth \\
\hline Roti $^{\circledR}$-Phenol/Chloroform/Isoamylalkohol & Roth \\
\hline SDS (Sodium dodecyl sulfate) & Sigma \\
\hline Streptavidin-biotinylated HRP & GE Healthcare \\
\hline SYBR GREEN I & Sigma Aldrich \\
\hline TEMED (N,N,N',N'-Tetramethylethan-1,2-diamin) & Roth \\
\hline Tris & Roth \\
\hline Triton X-100 & Sigma \\
\hline Trypan blue & Sigma \\
\hline Tween 20 & Sigma \\
\hline Xylene Cyanol & Roth \\
\hline Xylol & Roth \\
\hline$\beta$-Mercaptoethanol & Roth \\
\hline Dako EnVision Kit & DAKO \\
\hline
\end{tabular}




\begin{tabular}{l|l}
\hline Glycerin & Roth \\
\hline Ampicillin & Roth \\
\hline Bacto yeast extract & Roth \\
\hline $\mathrm{CaCl}_{2}$ (Calcium chloride) & Sigma \\
\hline Bacto tryptone & Becton Dickinson \\
\hline Agar-agar & Roth \\
\hline $5 \times$ Passive lysis buffer & Promega \\
\hline A-Sepharose & Sigma-Aldrich \\
\hline Flag-M2 Agarose from mouse & Sigma-Aldrich \\
\hline
\end{tabular}

Table 4: Commercial kits

\begin{tabular}{l|l} 
System & manufacturer \\
\hline \hline CloneJET ${ }^{\text {TM }}$ PCR Cloning Kit & Fermentas \\
\hline Fast Start Taq DNA Polymerase (dNTPs pack) & Roche \\
\hline GeneJET ${ }^{\text {TM Gel Extraction Kit }}$ & Fermentas \\
\hline Plasmid Plus Midi Kit & Qiagen \\
\hline Rapid DNA Ligation Kit & Fermentas \\
\hline TRI Reagent RNA Isolation Kit & Ambion \\
\hline GeneJET Plasmid Miniprep Kit & Thermo Scientific \\
\hline SYBR Green & Sigma-Aldrich \\
\hline Dako EnVision+ System HRP labeled & Dako EnVision+ System HRP labeled \\
\hline Human 4 $\times 44$ K design array 026652 & Agilent Technologies \\
\hline RNA Spike-In Kit for One color & Agilent Technologies \\
\hline Low RNA Input linear Amplification Kit Plus, One Color & Agilent Technologies \\
\hline
\end{tabular}


Table 5: Enzymes

\begin{tabular}{|c|c|}
\hline Enzyme & manufacturer \\
\hline coenzyme A & AppliChem \\
\hline DNase I, RNase-free & Fermentas \\
\hline HindIII & Fermentas \\
\hline HOT FIREPol DNA Polymerase & Solis BioDyne \\
\hline Luciferin & SYNCHEM \\
\hline Pstl & Fermentas \\
\hline EcoRI & Fermentas \\
\hline Phusion High-Fidelity DNA Polymerases & Thermo Scientific \\
\hline Proteinase $\mathrm{K}$ & Roche \\
\hline Reverse transcriptase, MMLV-RT & Fermentas \\
\hline RNase A & Roche \\
\hline Trypsin / EDTA & Invitrogen \\
\hline Xhol & Fermentas \\
\hline Shrimp Alkaline Phosphatase (SAP) & Fermentas \\
\hline BigDye $^{\circledR}$ Terminator v1.1 Cycle Sequencing Kit & Applied Biosystems \\
\hline
\end{tabular}

Table 6: Antibodies for Western Blots

\begin{tabular}{l|l|l|l|l} 
antibody & origin & dilution & product no. & source \\
\hline \hline $\begin{array}{l}\text { anti-Flag-HRP } \\
\text { anti-BCL9-2 }\end{array}$ & mouse & $1: 500$ & A8592 & Sigma \\
\hline anti- $\beta$-catenin & rabbit & $1: 100$ & self-made & $\begin{array}{l}\text { self-made (Brembeck et } \\
\text { al., 2011) }\end{array}$ \\
\hline $\begin{array}{l}\text { anti-CypA } \\
\text { anti- } \alpha \text {-tubulin }\end{array}$ & rabbit & $1: 1000$ & self-made & $\begin{array}{l}\text { self-made (Brembeck et } \\
\text { al., 2011) }\end{array}$ \\
\hline
\end{tabular}




\begin{tabular}{|c|c|c|c|c|}
\hline anti- $\beta$-actin & mouse & 1:10000 & A3853 & Sigma \\
\hline anti-laminB1 & goat & $1: 100$ & sc-6216 & Santa Cruz \\
\hline anti-CDX2 & rabbit & $1: 300$ & IHC-00126 & Bethyl \\
\hline anti-rabbit IgG HRP & goat & $1: 5000$ & $111-035-144$ & $\begin{array}{l}\text { Jackson } \\
\text { Immunoresearch }\end{array}$ \\
\hline anti-mouse IgG HRP & goat & $1: 5000$ & $115-035-062$ & $\begin{array}{l}\text { Jackson } \\
\text { Immunoresearch }\end{array}$ \\
\hline
\end{tabular}

Table 7: Antibodies for immunohistochemistry (IHC)

\begin{tabular}{|c|c|c|c|c|}
\hline antibody & origin & dilution & product no. & manufacturer \\
\hline anti-bromodeoxyuridine & rat & $1: 100$ & ab6326 & Abcam \\
\hline anti-Flag & rabbit & $1: 250$ & F7425 & Sigma \\
\hline anti-ß-catenin & rabbit & $1: 2000$ & self-made & $\begin{array}{l}\text { self-made (Brembeck et } \\
\text { al., 2011) }\end{array}$ \\
\hline anti-EPHB2 & goat & $1: 300$ & AF467 & R\&D Systems \\
\hline anti-EPHB3 & goat & $1: 300$ & AF432 & R\&D Systems \\
\hline anti-EPHB4 & goat & $1: 300$ & AF446 & R\&D Systems \\
\hline anti-Bambi & rabbit & $1: 250$ & self-made & $\begin{array}{l}\text { self-made (Brembeck et } \\
\text { al., 2011) }\end{array}$ \\
\hline anti-mouse IgG Cy2 & donkey & $1: 500$ & $715-226-150$ & $\begin{array}{l}\text { Jackson } \\
\text { Immunoresearch }\end{array}$ \\
\hline anti-rabbit IgG Cy3 & donkey & 1:1000 & $711-166-152$ & $\begin{array}{l}\text { Jackson } \\
\text { Immunoresearch }\end{array}$ \\
\hline anti-panCK & mouse & $1: 300$ & C2562 & Sigma \\
\hline anti- $\alpha \mathrm{SMA}$ & rabbit & 1:1500 & A2547 & Sigma \\
\hline anti-Cleaved caspase 3 & rabbit & 1:50 & $9661 \mathrm{~L}$ & Cell Signaling \\
\hline anti-rat Biotin IgG & goat & $1: 100$ & B7139 & Sigma \\
\hline
\end{tabular}


Table 8: Antibodies for Immunoprecipitation (IP)

\begin{tabular}{l|l|l|l|l} 
antibody & origin & dilution & product no. & manufacturer \\
\hline \hline anti-BCL9L (BCL9-2) & rabbit & & A303-152A & Biomol/Bethyl \\
\hline anti-SP1* & rabbit & $1: 1000$ & $07-645$ & millipore \\
\hline anti-PYGO2* & rabbit & $1: 1000$ & self-made & $\begin{array}{l}\text { self-made } \\
\text { (Brembeck et al., 2011) }\end{array}$
\end{tabular}

* also used for Western Blot

\subsection{Oligonucleotides and siRNA}

Oligonucleotides were synthesized by CIBA, Göttingen (Table 9 to Table 12). Restriction site sequences were introduced close to the $5^{\prime}$ end of primers used for cloning applications to flank the resulting product with restriction sites, if required. Oligonucleotides for quantitative realtime PCR were designed using PrimerQuest ${ }^{\mathrm{SM}}$ (Integrated DNA Technologies, Inc) or NCBI/Primer-BLAST, respectively. Primers were designed to span exon junctions when possible and to target all isoforms of one gene, if necessary and possible. The primer specificity was verified by nucleotide BLAST $^{\circledR}$ from NCBI. Self-dimerization, hetero-dimerization and hairpin structures of the oligos were analyzed using OligoAnalyzer 3.1 (Integrated DNA Technologies, Inc).

ON-TARGETplus siRNAs were obtained from Dharmacon, Bonn (Table 13).

Table 9: Oligonucleotides for mutagenesis PCR

\begin{tabular}{|c|c|}
\hline oligonucleotide/target & forward primer sequence $\left(5^{\prime}-3^{\prime}\right)$ \\
\hline -386bp hCDX1 Xhol for & ACTCGAGGTGAAGTTGGCCTAGAATCCCC \\
\hline +73bp hCDX1 HindIII rev & GATAAGCTTGCTGGGCCCTGGAGC \\
\hline -386bp hCDX1 Xhol -367bp BCL9 mt for & TAGCTCGAGGTGAAGTTGGCAGTGAATCCC \\
\hline hCDX1 -112bp NFkB mt for & CGACGGGTAGACCCCTTTGATTCG \\
\hline hCDX1 -112bp NFkB mt rev & CGAATCAAAGGGGTCTACCCGTCG \\
\hline hCDX1 -105bp TCF4 mt for & GTTTCCCCAGCTGATTCGCGG \\
\hline
\end{tabular}




\begin{tabular}{|c|c|}
\hline hCDX1 -105bp TCF4 mt rev & CCGCGAATCAGCTGGGGAAAC \\
\hline hCDX1 -66bp 0CT4 mt for & CCGCTTTGAAATAGCAACCCGCCTC \\
\hline hCDX1 -66bp OCT4 mt rev & GAGGCGGGTTGCTATTTCAAAGCGG \\
\hline hCDX1 -74bp TCF4 mt for & CTTCCCCCCGAGCTGAAATGC \\
\hline hCDX1 -74bp TCF4 mt rev & 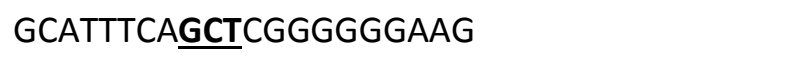 \\
\hline hCDX1 -8bp SP1-B for & TGGGTGGGGCAAGCGCGGC \\
\hline hCDX1 -8bp SP1-B rev & GCCGCGCTITGCCCCACCCA \\
\hline hCDX1 -137bp SP1-A for & CTTTTGAACCCCITTGCCCCCGAC \\
\hline hCDX1 -137bp SP1-A rev & GTCGGGGGCAAGGGGTTCAAAAG \\
\hline$-456 b p$ hCDX2 Xhol for & TAGCTCGAGGCCCGTTTCCAAACCCAGCTTC \\
\hline +142bp hCDX2 HindIII rev & GTAGGAGATCTTCTAGAAAGATAAGCTTGCTGC \\
\hline hCDX2 -367bp LEF $\mathrm{mt}$ for & 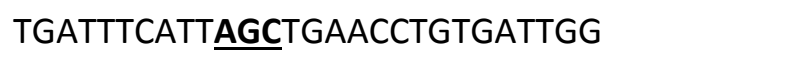 \\
\hline hCDX2 -367bp LEF mt rev & CCAATCACAGGTTCAGCTAATGAAATCA \\
\hline hCDX2 -234bp 0CT4 mt for & TCCССTTTATCTTTTAAAATTAGAATTATGTTTCGAG \\
\hline hCDX2 -234bp OCT4 mt rev & CTCGAAACATAATTCTAATTTTAAAAGATAAAGGGGA \\
\hline hCDX2 -219bp BCL6 mt for & 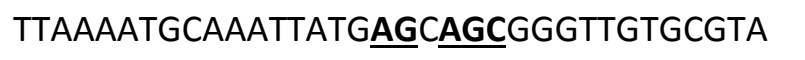 \\
\hline hCDX2 -219bp BCL6 mt rev & 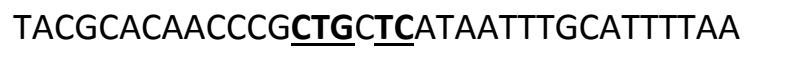 \\
\hline hCDX2 -170bp SMAD mt for & ACGTCTCCAACCATTGGTGA \\
\hline hCDX2 -170bp SMAD mt rev & TATTAGTAATGACACGCTCACCAATGGTTGGAGACGT \\
\hline hCDX2 -258bp NFkB mt for & ССTCTCACGAGCCAGACTCCCCTTTATCTT \\
\hline hCDX2 -258bp NFkB mt rev & AAGATAAAGGGGAGTCTGGCTCGTGAGAGG \\
\hline hCDX2 +95bp SMAD4 mt for & TGGGGCGCATGAACCCGCCGCT \\
\hline hCDX2 +95bp SMAD4 mt rev & AGCGGCGGGTICATGCGCCCCA \\
\hline hCDX2 -133bp CDX2 mt for & TCTTGTAAACACTCGTTCCTCACGGAAGG \\
\hline hCDX2 -133bp CDX2 mt rev & CCTTCCGTGAGGAACGAGTGTTTACAAGA \\
\hline hCDX2 -30bp SP1 mt for & GAAGAAAGGGATTGAGGGAGGAGGCAG \\
\hline hCDX2 -30bp SP1 mt rev & СTGССТССТСССТСААТСССТTTСТTC \\
\hline
\end{tabular}




\begin{tabular}{|c|c|}
\hline hCDX2 -266bp SP1 mt for & СТCTCACGAGCCTAАСТССССТTTATC \\
\hline hCDX2 -266bp SP1 mt rev & GATAAAGGGGAGITAGGCTCGTGAGAG \\
\hline hCDX2 -89bp SP1 mt for & CCTGTGGCTTGTCTTCCAAGCCTCTGCAG \\
\hline hCDX2 -89bp SP1 mt rev & CTGCAGAGGCTTGGAAGACAAGCCACAGG \\
\hline hCDX2 -121bp SP1 mt for & GTTAATCACGGAAGGCCGAAGGCCTGG \\
\hline hCDX2 -121bp SP1 mt rev & CCAGGCCTTCGGCCTTCCGTGATTAAC \\
\hline hCDX2 +65bp SP1 mt for & GCAGCCACAAGCCGCTCCTCGAGATCT \\
\hline hCDX2 +65bp SP1 mt rev & AGATCTCGAGGAGCGGCTTGTGGCTGC \\
\hline
\end{tabular}

Table 10: Oligonucleotides for sequencing reactions of Luciferase constructs

\begin{tabular}{l|l|l} 
oligonucleotide/target & primer sequence $\left(\mathbf{5}^{\prime} \mathbf{- 3} \mathbf{\prime}\right)$ & reference/source \\
\hline \hline pGL2Basic LUC intern rev & TGTAAAAGCAATTGTTCCAGGAACCAG & self-made \\
\hline pGL3Basic rev & CTTTATGTTTTTGGCGTCTTCCA & Promega \\
\hline pGL2Basic for & TGTATCTTAAGGTACTGTAACTG & Promega \\
\hline PJET for & CGACTCACTATAGGGAGAGCGGC & Fermentas \\
\hline pJET rev & AAGAACATCGATTTTCCATGGCAG & Fermentas
\end{tabular}

Table 11: Oligonucleotides for qPCR

\begin{tabular}{l|l|l}
$\begin{array}{l}\text { oligonucleotide/ } \\
\text { target }\end{array}$ & forward and reverse primer sequences (5'-3') & reference \\
\hline \hline \multirow{2}{*}{ hAXIN2 } & TCAAGTGCAAACTTTCGCCAACCG & self-made \\
\cline { 2 - 3 } & TGGTGCAAAGACATAGCCAGAACC & \\
\hline \multirow{2}{*}{ hBAMBI } & ATTCGATGCTACTGTGATGCTGCC & self-made \\
\cline { 2 - 3 } & ATTCCAATGTGGGTATGGTGGTGC & self-made \\
\hline \multirow{2}{*}{ hBMP4 } & TCTATGTGGACTTCAGCGATGTGG & \\
\cline { 2 - 3 } & AATTGACCAGGGTCTGCACAATGG & \\
\hline
\end{tabular}




\begin{tabular}{|c|c|c|}
\hline \multirow{2}{*}{$\mathrm{hCD} 44$} & ATCCCTCGGGTGTGCTATGGATGG & \multirow{2}{*}{ self-made } \\
\hline & CCTCAGTGGAAAGCAATGCCCAGG & \\
\hline \multirow{2}{*}{$\mathrm{h} C D \times 1$} & TAAGACTCGGACCAAGGACAAGTACC & \multirow{2}{*}{ self-made } \\
\hline & CTTGTTCACTTTGCGCTCCTTTGC & \\
\hline \multirow{2}{*}{$\mathrm{h} C D \times 2$} & TGGAGAAGGAGTTTCACTACAGTCGC & \multirow{2}{*}{ self-made } \\
\hline & TCTGGGACACTTCTCAGAGGACC & \\
\hline \multirow{2}{*}{$\mathrm{h} M Y C$} & TCTCCACACATCAGCACAACTACG & \multirow{2}{*}{ self-made } \\
\hline & TGTGTTCGCCTCTTGACATTCTCC & \\
\hline \multirow{2}{*}{$\mathrm{h} E F N B 3$} & GGAGGTGGATGGTTCTTATTCTGTGG & \multirow{2}{*}{ self-made } \\
\hline & ACATGGCAGTCATCTTAGCTGTCC & \\
\hline \multirow[t]{2}{*}{$\mathrm{h} E F N B 2$} & TTTGCAGGGATTGCTTCAGGATGC & \multirow{2}{*}{ self-made } \\
\hline & TTAGCGGGATGATAATGTCACTGGGC & \\
\hline \multirow{2}{*}{$\mathrm{h} E F N B 1$} & TGATGATGAGCAGGAAGATGACGC & \multirow{2}{*}{ self-made } \\
\hline & ATGGCAAGCATGAGACTGTGAACC & \\
\hline \multirow{2}{*}{$\mathrm{h} E P H B 2$} & CGCCATCTATGTCTTCCAGGTGCG & \multirow{2}{*}{ self-made } \\
\hline & CTCCGAGTCAGCACGCTCAAACC & \\
\hline \multirow[t]{2}{*}{$\mathrm{h} E P H B 3$} & TGGTGCTGTCATAAAAGGGCAGGC & \multirow{2}{*}{ self-made } \\
\hline & CCTTTATTCTGCTGTCGGCCCAGC & \\
\hline \multirow{2}{*}{$\mathrm{h} E P H B 4$} & GCCAGTGTCCAGCACATGAAGTCC & \multirow{2}{*}{ self-made } \\
\hline & AAATTGCCAАСТССТСАССССАCG & \\
\hline \multirow{2}{*}{$\mathrm{h} L E F 1$} & ACCTCAGGTCAAACAGGAACATCC & \multirow{2}{*}{ self-made } \\
\hline & AGTACACTCAGCAACGACATTCGC & \\
\hline \multirow[t]{2}{*}{$\mathrm{hTCF1}$} & GAACATTTCAACAGCCCACATCCC & \multirow{2}{*}{ self-made } \\
\hline & ACCAGAACCTAGCATCAAGGATGG & \\
\hline \multirow{2}{*}{$\mathrm{mBc} / 9-2$} & AATCATGGCAAGACAGGGAATGGC & \multirow{2}{*}{ self-made } \\
\hline & TCTTCAGACTTGAGTTGCTAGGCG & \\
\hline
\end{tabular}




\begin{tabular}{|c|c|c|}
\hline \multirow{2}{*}{$\begin{array}{l}\text { hCTNNB1 } \\
\text { (ß-catenin) }\end{array}$} & TTCGAAATCTTGCCCTTTGTCCCG & \multirow{2}{*}{ self-made } \\
\hline & AATTCGGTTGTGAACATCCCGAGC & \\
\hline \multirow{2}{*}{$\begin{array}{l}\mathrm{h} A C T B / \mathrm{h} A c b t \\
\text { (ß-actin) }\end{array}$} & ATAGCACAGCCTGGATAGCAACGTAC & \multirow{2}{*}{ (West et al., 2004) } \\
\hline & САССTTCTACAATGAGCTGCGTGTG & \\
\hline \multirow[t]{2}{*}{$\mathrm{h} B C L 9$} & ACCACATAAAGTCCCAGGATTCCC & \multirow{2}{*}{ self-made } \\
\hline & AAACACGTACACCACTTTGGCTGG & \\
\hline \multirow{2}{*}{$\mathrm{h} M S \times 2$} & AAATCTGGTTCCAGAACCGAAGGG & \multirow{2}{*}{ (Diecke et al., 2008) } \\
\hline & ATGGGAAGCACAGGTCTATGGAACG & \\
\hline \multirow{2}{*}{$\mathrm{hSOX9}$} & CAGCGAACGCACATCAAGACGGA & \multirow{2}{*}{ self-made } \\
\hline & GCTGGAGTTCTGGTGGTCGGTGTA & \\
\hline \multirow[t]{2}{*}{ hSOX6 } & GCAACTACCACACCATCGCCTC & \multirow{2}{*}{ self-made } \\
\hline & TAGGCTTCCGCCATCTGTCTTC & \\
\hline \multirow{2}{*}{$\mathrm{hSOX} 4$} & CCCGGACTACTGCACGCC & \multirow{2}{*}{ self-made } \\
\hline & CGAGAACGCGGGCGAACGA & \\
\hline \multirow{2}{*}{ hOCT4 } & GAGAACCGAGTGAGAGGCAACC & \multirow{2}{*}{ (Willems et al., 2006) } \\
\hline & CATAGTCGCTGCTTGATCGCTTG & \\
\hline \multirow[t]{2}{*}{ hCYPA } & CCCACCGTGTTCTTCGACAT & \multirow{2}{*}{ (Murphy et al., 2003) } \\
\hline & CCAGTGCTCAGAGCACGAAA & \\
\hline \multirow{2}{*}{$\mathrm{hSDNSF}$} & TCAACAAACCAGAGGCGGAGATGT & \multirow{2}{*}{ self-made } \\
\hline & TTAGTGGTGCCTGTTCACTCCCTT & \\
\hline \multirow[t]{2}{*}{$\mathrm{h} L G R 5$} & ACACATTGCCCTGTTGCTCTTCAC & \multirow{2}{*}{ self-made } \\
\hline & TTTCTCAGGCTCACCAGATCCTCC & \\
\hline \multirow{2}{*}{$\mathrm{h} B C L 9-2$} & АТСССТССАААССАТАAАGСАССС & \multirow{2}{*}{ self-made } \\
\hline & TGGAACTGGGCATTGCAAACTTGG & \\
\hline \multirow{2}{*}{$\begin{array}{l}\text { hCCND1 } \\
\text { (CyclinD1) }\end{array}$} & TGGTGAACAAGCTCAAGTGGAACC & \multirow{2}{*}{ self-made } \\
\hline & ATGGAGGGCGGATTGGAAATGAAC & \\
\hline
\end{tabular}




\begin{tabular}{l|l|l}
\hline \multirow{2}{*}{ hPROX1 } & AGAGCCTCCCATTACTCAGACCCG & self-made \\
\cline { 2 - 3 } & TGGTCAGGCATCACTGGACGGT &
\end{tabular}

Table 12: Oligonucleotides for conventional PCR

\begin{tabular}{l|l|l} 
oligonucleotide/target & primer sequence $\left.\quad \mathbf{( 5}^{\prime} \mathbf{- 3} \mathbf{\prime}\right)$ & \\
\hline \hline BCL9-2 intron-TG for & CTGGTCATCATCCTGCCTTT & self-made \\
\hline BCL9-2 exon1 for & TCCTGGCTAACAAGACAAGG & self-made \\
\hline BCL9-2 exon2 rev & CTCTGAATCGAGGGATGGAG & self-made \\
\hline MIN-wt for & GCCATCCCTTCACGTTAG & self-made \\
\hline MIN-wt rev & TTCCACTTTGGCATAAGGC & self-made \\
\hline MIN-mt for & TTCTGAGAAAGACAGAAGTTA & self-made \\
\hline ß-actin for & TGGCACCACACCTTCTACAATGAGC & self-made \\
\hline B-actin rev & GCACAGCTTCTCCTTAATGTCACGC & self-made \\
\hline
\end{tabular}

Table 13: ON-TARGETplus siRNA (Dharacon)

\begin{tabular}{|c|c|c|}
\hline siRNA/target & ID-No. & sequence $\left(5^{\prime}-3^{\prime}\right)$ \\
\hline ON-TARGETplus Non-targeting Pool & & not provided \\
\hline \multirow{4}{*}{ Human CTNNB1 (ß-catenin) } & 003482-09 & GAUCCUAGCUAUCGUUCUU \\
\hline & $003482-10$ & UUAAUGAGGACCUAUACUUA \\
\hline & $003482-11$ & GCGUUUGGCUGAACCAUCA \\
\hline & $003482-12$ & GGUACGAGCUGCUAUGUUC \\
\hline \multirow{4}{*}{ Human BCL9L (BCL9-2) } & 010858-05 & GCUCAUGCCUUCACAGUUU \\
\hline & 010858-06 & GAAAGCCUCCCUCGCAGUU \\
\hline & 010858-07 & CACCCACAAUUGUAAUGUA \\
\hline & 010858-08 & AACCAGAUCUCGCCUAGCA \\
\hline
\end{tabular}




\begin{tabular}{l|l|l|}
\hline \multirow{4}{*}{ Human $B C L 9$} & $007268-05$ & CUACUGAGUAGGCCAAUAA \\
\hline $007268-06$ & CCAAAGUGGUGUACGUGUU \\
\hline $007268-07$ & GCGGAAGCCCUUGGAUAUA \\
\hline \multirow{2}{*}{$007268-08$} & UUUGAUCUAUCCCGCAUUA
\end{tabular}

\subsection{Cell biology}

\subsubsection{Bacterial strains}

For cloning procedures and plasmid preparation the chemo-competent E. coli strain One Shot ${ }^{\circ}$ Top10F' (Invitrogen, Darmstadt) with the following genotype was used: F'\{lacla Tn10 (TetR) mcrA $\Delta$ (mrr-hsdRMS-mcrBC) Ф80lacZDM15 $\Delta$ lacX74 recA1 araD139 $\Delta$ (araleu)7697 galU galK rpsL endA1 nupG.

\subsubsection{Cell lines and mouse strains}

Cell lines were obtained from ATCC and LGC (LGC Standards GmbH, Wesel) and listed in Table 14. Utilized mouse strains are listed in Table 15.

Table 14: Cell lines

\begin{tabular}{l|l} 
cell line & Description
\end{tabular}

\begin{tabular}{l|l}
\hline \hline SW480 & $\begin{array}{l}\text { Colon cancer cell line established from a primary adenocarcinoma of the } \\
\text { colon of a } 50 \text { years old Caucasian male (Leibovitz et al., 1976). }\end{array}$ \\
\hline SW620 & $\begin{array}{l}\text { Colorectal adenocarcinoma isolated from the tissue (lymph node metastasis) } \\
\text { of a } 51 \text { years old Caucasian male as was SW480 (Leibovitz et al., 1976). }\end{array}$ \\
CACO2 & $\begin{array}{l}\text { Colorectal adenocarcinoma cell line derived from a } 72 \text { years old Caucasian } \\
\text { male (Fogh et al., 1977b). }\end{array}$ \\
CT26 & $\begin{array}{l}\text { N-nitroso-N-methylurethane-(NNMU), undifferentiated induced colon } \\
\text { carcinoma cell line derived from BALB/c mice (Wang et al., 1995). }\end{array}$ \\
HT29 & $\begin{array}{l}\text { Epithelial colorectal adenocarcinoma cell line from a } 44 \text { years old Caucasian } \\
\text { woman (Fogh et al., 1977a). }\end{array}$
\end{tabular}




\begin{tabular}{|c|c|}
\hline DLD1 & $\begin{array}{l}\text { Epithelial colorectal adenocarcinoma derived from an adult female (Dexter } \\
\text { et al., 1979). }\end{array}$ \\
\hline HCT116 & $\begin{array}{l}\text { Colorectal carcinoma cell line obtained from an adult male (Brattain et al., } \\
\text { 1981). }\end{array}$ \\
\hline WiDr & $\begin{array}{l}\text { Colon adenocarcinoma line from a } 78 \text { year old female, derivative of HT-29 } \\
\text { (Chen et al., 1987; Noguchi et al., 1979). }\end{array}$ \\
\hline SW48 & $\begin{array}{l}\text { Colon cancer cell line established from a primary adenocarcinoma of the } \\
\text { colon of a } 82 \text { years old Caucasian female (Leibovitz et al., 1976). }\end{array}$ \\
\hline Lovo & $\begin{array}{l}\text { initiated in } 1971 \text { from a fragment of a metastatic tumor nodule in the left } \\
\text { supraclavicular region of a } 56 \text { years old Caucasian male patient with a } \\
\text { adenocarcinoma of the colon (Drewinko et al., 1976). }\end{array}$ \\
\hline Ls174T & $\begin{array}{l}\text { variant of LS 180, which is a colorectal adenocarcinoma cell line from a } 58 \\
\text { years old Caucasian woman (Tom et al., 1976). }\end{array}$ \\
\hline HEK293 & Human embryonic kidney cell line (Graham et al., 1977). \\
\hline MCF7 & $\begin{array}{l}\text { Breast cancer cell line isolated in } 1970 \text { from a } 69 \text { years old Caucasian woman } \\
\text { (Soule et al., 1973). }\end{array}$ \\
\hline
\end{tabular}

Table 15: Utilized mouse strains

\begin{tabular}{l|l} 
mouse strain & Description \\
\hline \hline APC $^{\text {Min/+ }}$ & $\begin{array}{l}\text { Derived from an N-ethyl-N-nitrosourea (ENU) treated founder C57BL/6J } \\
\text { mice, that induced a point mutation (T to A transversion of nucleotide 2549) } \\
\text { in the APC gene. Already young mice are highly susceptible to spontaneous } \\
\text { intestinal adenoma formation (Su et al., 1992). (Jackson Laboratories) }\end{array}$ \\
\hline K19-BCL9-2 & $\begin{array}{l}\text { Transgenic mice express BCL9-2 under control of a K19 promoter in simple } \\
\text { epithelia, including the intestine, mammary glands and pancreas (Brembeck } \\
\text { et al., 2011). }\end{array}$
\end{tabular}




\subsection{Plasmids}

Table 16: Luciferase-reporter plasmids

\begin{tabular}{|c|c|}
\hline Plasmid & source /reference \\
\hline ptkRenilla & Promega \\
\hline TOPflash/FOPflash & $\begin{array}{l}\text { R.T Moon, Seattle } \\
\text { (Korinek et al., 1997) }\end{array}$ \\
\hline pGL2Basic & Promega \\
\hline pGL3Basic & Promega \\
\hline pGL3Basic $-5,6 \mathrm{~kb}$ Axin2 & $\begin{array}{l}\text { F. Constantini, New York } \\
\text { (Jho et al., 2002) }\end{array}$ \\
\hline pA3 LUC Cyclin D1 & $\begin{array}{l}\text { R. Pestell, Philadelphia } \\
\text { (Shtutman et al., 1999) }\end{array}$ \\
\hline pGL2Basic -3189bp CDX1 & $\begin{array}{l}\text { J. Lynch, Philadelphia } \\
\text { (Suh et al., 2002) }\end{array}$ \\
\hline pGL2Basic -386bp CDX1 & $\begin{array}{l}\text { J. Lynch, Philadelphia } \\
\text { (Suh et al., 2002) }\end{array}$ \\
\hline pGL2Basic -1434bp CDX2 & $\begin{array}{l}\text { J. Lynch, Philadelphia } \\
\text { (unpublished) }\end{array}$ \\
\hline pGL2Basic $-456 b p$ CDX2 & $\begin{array}{l}\text { J. Lynch, Philadelphia } \\
\text { (unpublished) }\end{array}$ \\
\hline pGL2Basic -281bp CDX2 & $\begin{array}{l}\text { J. Lynch, Philadelphia } \\
\text { (unpublished) }\end{array}$ \\
\hline pGL2Basic -386bp CDX1 -377BCL6-BS mt & this work \\
\hline pGL2Basic -386bp CDX1 -122NFkB-BS mt & this work \\
\hline pGL2Basic -386bp CDX1 -113LEF-BS mt & this work \\
\hline pGL2Basic -386bp CDX1 -810CT4-BS mt & this work \\
\hline pGL2Basic -386bp CDX1 -82LEF-BS mt & this work \\
\hline pGL2Basic -386bp CDX1 -82LEF-TF /-113LEF-BS mt & this work \\
\hline pGL2Basic -386bp CDX1 -8SP1-BS mt & this work \\
\hline
\end{tabular}




\begin{tabular}{|c|c|}
\hline pGL2Basic -386bp CDX1 -137SP1-BS mt & this work \\
\hline pGL2Basic -386bp CDX1-8SP1-BS mt/-137SP1-BS & this work \\
\hline pGL2Basic -456bp CDX2 -229BCL6-BS mt & this work \\
\hline pGL2Basic -456bp CDX2 -373LEF-BS mt & this work \\
\hline pGL2Basic -456bp CDX2 -2400CT4-BS mt & this work \\
\hline pGL2Basic -456bp CDX2 -264NFkB-BS mt & this work \\
\hline pGL2Basic -456bp CDX2 -175Smad-BS mt & this work \\
\hline pGL2Basic $-456 b p$ CDX2 +62Smad-BS mt & this work \\
\hline pGL2Basic -456bp CDX2 -132CDX2-BS mt & this work \\
\hline pGL2Basic -456bp CDX2 -30bp SP1-BS mt & this work \\
\hline pGL2Basic -456bp CDX2 -266bp SP1-BS mt & this work \\
\hline pGL2Basic -456bp CDX2 -89bp SP1-BS mt & this work \\
\hline pGL2Basic -456bp CDX2 -121bp SP1-BS mt & this work \\
\hline pGL2Basic -456bp CDX2 +65bp SP1-BS mt & this work \\
\hline pGL2Basic -456bp CDX2 -30bp SP1-BS mt/-266 SP1-BS mt & this work \\
\hline
\end{tabular}

Table 17: Eukaryotic overexpression plasmids

\begin{tabular}{l|l} 
Plasmid & source/reference \\
\hline \hline pcDNA-flag & Self-made (Brembeck et al., 2004) \\
pcDNA-HA hOCT4 & $\begin{array}{l}\text { S. Monecke / R. Dressel, Göttingen } \\
\text { (unpublished) }\end{array}$ \\
\hline
\end{tabular}

\subsection{Buffers and solutions}

Standard buffers and solutions were prepared as described in (Michael R.Green et al., 2012). The compositions of additional buffers and solutions are listed in the corresponding method sections. For sterilization, solutions were autoclaved $\left(120^{\circ} \mathrm{C}\right.$ for $\left.60 \mathrm{~min}, 2 \mathrm{bar}\right)$ or filtrated with a $0.2 \mu \mathrm{m}$ sterile filter. 


\subsection{Microbiology}

\subsubsection{Cultivation and storage of $E$. coli}

For glycerin stocks and low scale plasmid preparations which required small volumes of $E$. coli bacterial suspensions $2 \mathrm{ml}$, for large scale plasmid preparations $250 \mathrm{ml}$ LB medium was supplemented with Ampicillin antibiotics. E. coli bacterial suspensions were cultured $\mathrm{o} / \mathrm{n}$ at $37^{\circ} \mathrm{C}$ in an incubator shaker at $220 \mathrm{rpm}$.

For long-term storage $700 \mu \mathrm{l} \mathrm{E}$. coli suspension was vigorously mixed with $300 \mu \mathrm{l} 100 \%$ glycerin and stored at $-80^{\circ} \mathrm{C}$.

\subsubsection{Generation of chemo-competent $E$. coli}

$200 \mathrm{ml} \mathrm{LB}$-medium was inoculated with $5 \mathrm{ml}$ of an o/n E. coli suspension. The culture was incubated until an $\mathrm{OD}_{600}$ of 0.5 to 0.6 was reached. The bacterial suspension was incubated on ice for $10 \mathrm{~min}$ and afterwards centrifuged for $10 \mathrm{~min}$ at $4^{\circ} \mathrm{C}$ with $4600 \mathrm{rpm}$. The resulting pellets were resolved in $80 \mathrm{ml} 100 \mathrm{mM} \mathrm{CaCl}_{2}$ and incubated on ice for further $10 \mathrm{~min}$. Following an additional centrifugation step E. coli pellets were resolved in $8 \mathrm{ml} 100 \mathrm{mM} \mathrm{CaCl}$, supplemented with $600 \mu \mathrm{l}$ glycerin and incubated on ice for $15 \mathrm{~min}$. Aliquots of $100 \mu \mathrm{l}$ competent bacterial suspensions were frozen in liquid nitrogen and stored at $-80^{\circ} \mathrm{C}$.

\subsubsection{Transformation of $E$. coli}

Plasmid DNA was introduced in E. coli bacteria using heat shock. Therefore, $10 \mu$ ligation reaction was added to $50 \mu \mathrm{l}$ of chemo-competent $E$. coli bacterial solution. Following $45 \mathrm{~min}$ of incubation on ice the transformation mix was set to $42^{\circ} \mathrm{C}$ for $60 \mathrm{~s}$. After the solution was cooled down on ice $500 \mu \mathrm{l} \mathrm{SOC}$ medium were added and incubated at $37^{\circ} \mathrm{C}$ and $330 \mathrm{rpm}$ for 60 min. The mixture was then plated on $L B$ agar plates and cultivated $\mathrm{o} / \mathrm{n}$ at $37^{\circ} \mathrm{C}$. 


\subsection{Molecular biology}

\subsubsection{Isolation of nucleic acids}

\subsubsection{Isolation of genomic DNA from tails of transgenic mice}

Genomic DNA from mouse tails was used for genotyping transgenic mice. $2 \mathrm{~mm}$ of a severed mouse tail tip was lysed by adding $50 \mu \mathrm{l}$ Lysis buffer followed by o/n incubation at $65^{\circ} \mathrm{C}$ and $500 \mathrm{rpm}$. The mixture was diluted to a final volume of $500 \mu \mathrm{l}$ and centrifuged with maximum speed. 1 to $2 \mu$ l supernatant was used for genotyping PCR (2.7.3.1).

Lysis buffer

$100 \mathrm{mM}$ Tris- $\mathrm{HCl}$ pH 8.5, 5 mM EDTA pH 8.0, $200 \mathrm{mM} \mathrm{NaCl}, 0.2 \%$ (w/v) SDS, $200 \mu \mathrm{g} / \mathrm{ml}$ Proteinase $\mathrm{K}$

\subsubsection{Isolation of total RNA}

Cells were seeded in 6-well plates (according to Table 18) for RNA isolation from cell cultures and transfected and incubated as described in 3.9.3.2. Cells were lysed by adding $1 \mathrm{ml}$ TRI Reagent (Ambion, Darmstadt). For isolation of RNA from mouse tissues TRI Reagent was added to fresh tissues according to manufacturer's instructions. Cells and tissues were frozen in liquid nitrogen for storage at $-80^{\circ} \mathrm{C}$ or homogenized, respectively. Total RNA was isolated using the TRI Reagent RNA Isolation Kit according to manufacturer's instructions. To avoid contamination of RNA with genomic DNA, RNA isolated from mouse tissues was digested with DNasel (Fermentas, St. Leon-Rot) for $1.5 \mathrm{~h}$ according to manufacturer's instruction. RNA was then purificated according to 3.2.2.2. The quality and quantity of RNA was determined using NanoDrop and Agarose gel electrophoresis.

\subsubsection{Isolation of plasmid DNA from E. coli}

Plasmid DNA was isolated from o/n E.coli bacterial suspensions (2.6.1). The GeneJET Plasmid Miniprep Kit (Fermentas, St. Leon-Rot) was used for cloning procedures, the Plasmid plus Midi Kit (Qiagen, Hilden) for isolation of large amount of plasmid DNA according to manufacturer's instructions. DNA was dissolved in $\mathrm{dH} 2 \mathrm{O}$ and stored at $-20^{\circ} \mathrm{C}$. 


\subsubsection{Purification and extraction of nucleic acids}

\subsubsection{Extraction of nucleic acids from agarose gels}

DNA fragments were extracted and purified from agarose gels using the GeneJET'M Gel Extraction Kit (Fermentas, St. Leon-Rot) according to the manufacturer's instructions.

\subsubsection{Phenol chloroform extraction}

After DNase digestion (3.7.1.2) RNA solutions were purified from proteins and other contaminants using phenol chloroform extraction. Therefore 1 volume of RNA solution was consecutively mixed thoroughly with 1 volume phenol chloroform isoamylalcohol solution. The solution was centrifuged at $4^{\circ} \mathrm{C}$ with $10500 \mathrm{rpm}$ and the aqueous phase was transferred into a new reaction tube. Phenol remains were removed by adding ammonium acetate to a final concentration of $2.5 \mathrm{M}$ and precipitated with $100 \%$ Ethanol (70\% final) and a subsequent washing step with $1.5 \mathrm{ml} 70 \%$ Ethanol.

\subsubsection{Amplification of nucleic acids}

\subsubsection{Detection of genomic DNA and transcripts by Polymerase chain reaction (PCR)}

Genotypes of transgenic mice and the expression of transgenic BCL9-2 and ß-actin were examined by PCR using specific primers (Table 12) and the following cycling conditions

for genotyping of K19-BCL9-2 mice, for genotyping of $\mathrm{APC}^{\mathrm{MIN} /+}$ mice expression of TG-BCL9-2 and ß-actin mRNA

\begin{tabular}{|c|c|c|c|c|}
\hline $95^{\circ} \mathrm{C}$ & $10 \mathrm{~min}$ & & $95^{\circ} \mathrm{C}$ & $10 \mathrm{~min}$ \\
\hline $95^{\circ} \mathrm{C}$ & $30 \mathrm{~s}$ & & $95^{\circ} \mathrm{C}$ & $30 \mathrm{~s}$ \\
\hline $60^{\circ} \mathrm{C}$ & $30 \mathrm{~s}$ & $35 x$ & $45^{\circ} \mathrm{C}$ & $30 \mathrm{~s}$ \\
\hline $72^{\circ} \mathrm{C}$ & $150 \mathrm{~s}$ & & $72^{\circ} \mathrm{C}$ & $150 \mathrm{~s}$ \\
\hline $72^{\circ} \mathrm{C}$ & $10 \mathrm{~min}$ & & $72^{\circ} \mathrm{C}$ & $10 \mathrm{~min}$ \\
\hline
\end{tabular}

For analytical proposes a reaction was set up as follows:

1x Taq buffer, $2.5 \mathrm{mM} \mathrm{MgCl}$, $0.2 \mu \mathrm{M}$ primer each, $0.2 \mathrm{mM}$ dNTPs each, 0.6 units FastTaq DNA Polymerase and in a final volume of $15 \mu \mathrm{l}$ per reaction. $1 \mu \mathrm{l}$ of genomic mouse tail DNA (see 3.2.1.1) or $10 \mathrm{ng}$ (for $\beta$-actin) to $500 \mathrm{ng}$ (transgenic BCL9-2) cDNA from mouse tissues (see 
3.2.3.2.) were used as templates. Instead, addition of $\mathrm{H}_{2} \mathrm{O}$ was served as negative control. The resulting products were analyzed in $1 \%$ agarose gels.

\subsubsection{Mutagenesis Polymerase chain reaction (mt-PCR)}

Single mutations were introduced in the promoters of Luciferase-reporters by mutagenesis PCR. Therefore forward and reverse oligonucleotides were used, which carry the final mutation. In a first step 2 PCR reactions were performed to introduce the mutated transcription factor binding site. To this end, forward mutagenesis primers and reverse primers targeting the $5^{\prime}$ end of the promoter were used. The second PCR reaction was performed with forward primers targeting the $3^{\prime}$ end of the promoter and reverse mutagenesis primers (Figure 9A). In a second step the amplified products of step 1 were used as templates for PCR. In addition, forward primers targeting the $3^{\prime}$ - and reverse primers targeting the $5^{\prime}$ end of the promoter were added to amplify the final product (Figure 9B).

A. Step1 wildtype promoter in pGL2Basic / template step 1

\begin{tabular}{|c|c|c|c|}
\hline \multirow{3}{*}{ PCR1 } & & ...GCTCG... & \\
\hline & Xhol & AGA & \\
\hline & $3^{\prime}$ forward & $\mathrm{mt}$ reverse & \\
\hline \multirow{2}{*}{ PCR2 } & & TCT & HindIII \\
\hline & & $\mathrm{mt}$ forward & \\
\hline
\end{tabular}

B. Step2 products step 1 / template step 2

\begin{tabular}{|c|c|c|c|}
\hline & Xhol & ...GAGAG... & \\
\hline & & ...СТСТС... & HindIII \\
\hline & Xhol & & HindIII \\
\hline & $3^{\prime}$ forward & & erse \\
\hline
\end{tabular}

products step $2 /$ mutated promoter fragment

\begin{tabular}{|lll|}
\hline Xhol & ...GAGAG... HindIII \\
\hline
\end{tabular}

Figure 9: Scheme of mutagenesis PCR to indroduce mutations in the promoters of CDX1 and CDX2.

For preparative proposes Phusion polymerase was used, since this enzyme exhibits proof reading activity. Phusion driven reactions were set up as follows:

$1 \times$ Phusion PCR buffer, $0.8 \mathrm{mM}$ dNTP-Mix, 10 pmol primers each, $2 \mathrm{U}$ Phusion polymerase and $100 \mathrm{ng}$ template in a final volume of $50 \mu \mathrm{l}$. 
Cycle conditions for mutagenesis PCR were set as shown in the following. Thereby the melting temperature $T_{M}$ of the used oligonucleotides (Table 9) was calculated as described in 2.2

$\left.\begin{array}{ll}95^{\circ} \mathrm{C} & 10 \mathrm{~min} \\ 92^{\circ} \mathrm{C} & 15 \mathrm{~s} \\ 50^{\circ} \mathrm{C} & 15 \mathrm{~s} \\ 72^{\circ} \mathrm{C} & 30 \mathrm{~s} \\ 95^{\circ} \mathrm{C} & 15 \mathrm{~s} \\ 60^{\circ} \mathrm{C} & 15 \mathrm{~s} \\ 72^{\circ} \mathrm{C} & 30 \mathrm{~s} \\ 72^{\circ} \mathrm{C} & 10 \mathrm{~min}\end{array}\right] 35 \mathrm{x}$

\subsubsection{Synthesis of copy DNA (cDNA)}

RNA samples were transcribed into cDNA for $\mathrm{qPCR}$ and PCR. To this end, the Reverse transcriptase (RT), MMLV-RT system (Fermentas, St. Leon-Rot) was used and adapted as described.

For cDNA synthesis $2.5 \mu \mathrm{g}$ RNA derived from cell cultures or $7.5 \mu \mathrm{g}$ RNA isolated from mouse tissues were added to $3 \mathrm{pmol}$ random hexamer primer in a total volume of $31 \mu \mathrm{l}$. $30 \mu \mathrm{l}$ were used as cDNA sample, $1 \mu$ l was saved as $-\mathrm{RT}$ control. After an initial incubation of the samples at $65^{\circ} \mathrm{C}$ for $5 \mathrm{~min}$ the following master mix was added to a final volume of $60 \mu \mathrm{l}: 1 \mathrm{x} \mathrm{MMLV}$ RTase buffer, 20 mM DTT, 60 U RNase Out, 10 mM dNTPs, 150 U MMLV reverse transcriptase. The samples were subsequently incubated for $10 \mathrm{~min}$ at $25^{\circ} \mathrm{C}$, followed by $60 \mathrm{~min}$ at $37^{\circ} \mathrm{C}$ and $10 \mathrm{~min}$ at $70^{\circ} \mathrm{C}$. The resulting CDNA was stored at $-20^{\circ} \mathrm{C}$.

\subsubsection{Quantitative real-time PCR (qPCR)}

Depending on the expression level of the transcript of interest $15 \mathrm{ng}$ to $30 \mathrm{ng}$ of cDNA was used per sample. The cDNA was added to 3 pmol oligonucleotides and 7,5 $\mu$ I SYBRGreen PCR Master Mix to a final volume of $10 \mu \mathrm{l}$. To investigate the transcription of specific mRNAs oligonucleotides with and efficiency of $2 \pm 0.08$ and an annealing temperature of $60^{\circ} \mathrm{C} \pm 1^{\circ} \mathrm{C}$ were used, which resulted in the following cycle conditions: $12 \mathrm{~min} 95^{\circ} \mathrm{C}$, followed by 40 cycles of $30 \mathrm{~s}$ at $95^{\circ} \mathrm{C}, 30 \mathrm{~s}$ at $60^{\circ} \mathrm{C}, 30 \mathrm{~s}$ at $72^{\circ} \mathrm{C}$ and subsequently $10 \mathrm{~min}$ at $72^{\circ} \mathrm{C}$. A melting curve was added consisting of an initial $95^{\circ} \mathrm{C}$ denaturation step for $15 \mathrm{~s}$, followed by $60^{\circ} \mathrm{C}$ for $30 \mathrm{~s}$ and a gradually heating step to $95^{\circ} \mathrm{C}$.

SYBR Green PCR $\quad 75$ mM Tris-HCl pH 8.8, 20 mM (NH4)2SO4, 0.01\% Tween-20, 3 mM Master Mix $\quad \mathrm{MgCl} 2,0.2 \mathrm{mM}$ dNTP's, $20 \mathrm{U} / \mathrm{ml}$ HOT FIREPol DNA Polymerase, $0.25 \%$ TritonX-100, 500 mM D(+)-Trehalose Dihydrat, 1:80000 (v/v) Cybr 
Green

Finally, the relative expression of target genes was calculated by $2^{-\Delta \Delta C t}$ (Fleige et al., 2006). The composition of the SYBR Green PCR Master Mix was kindly provided by Prof. Dr. Steven Johnsen, Dept. of Molecular Oncology, Georg August University Göttingen, Germany.

\subsubsection{Molecular cloning}

The purified CDX1 and CDX2 promoter fragments generated by mutagenesis PCR (3.7.3.2) were digested using the added restriction sites Xhol and HindIII (3.7.4.1) and subsequently subcloned into pJET2.1 (Fermentas, St. Leon-Rot) (3.7.4.3). Finally, the promoter fragments were cloned from pJET2.1 into pGL2Basic. For CDX1 promoters, Xhol/HindIII were used, while for CDX2 promoter fragments Xhol was used. In parallel, pGL2Basic vectors were restricted with respect to their prospective insert and dephosphorylated. The linear fragments were separated from the vector backbone or non-digested vector remains, respectively, by gelelectrophoresis and purified from the gels (3.7.2.1). After ligation (3.7.4.3) the successful insertion of the mutated CDX1 and CDX2 promoter fragments in pGL2Basic was finally checked by sequencing using the BigDye ${ }^{\circledR}$ Terminator v1.1 Cycle Sequencing Kit (Applied Biosystems, Carlsbad, California) according to manufacturer's instructions using specific primers listed in Table 10 and test restriction (3.7.4.1).

\subsubsection{Restriction of nucleic acids with restriction endonucleases}

DNA-fragments and plasmids were restricted according to DoubleDigest ${ }^{\mathrm{TM}}$ standard protocols available from the Fermentas web page (http://www.thermoscientificbio.com/webtools/doubledigest/). CDX1 promoter containing pGL2Basic plasmids were resticted in R-buffer with ECoRI and Xhol, CDX2 promoter containing pGL2Basic vectors were restricted in O-buffer with EcoRI and Xhol (Fermentas, St. Leon-Rot).

\subsubsection{Dephosphorylation of nucleic acids}

Phosphate residues were removed from pGL2Basic vectors using calf intestine phosphatase (CIP). To this end, $10 \mathrm{U}$ of Shrimp Alkaline Phosphatase (SAP) was added to $10 \mu \mathrm{g}$ restriction digest and incubated for $60 \mathrm{~min}$ at $37^{\circ} \mathrm{C}$.

\subsubsection{Ligation of DNA fragments}

For sub-cloning of mutated CDX1 and CDX2 promoter fragments into pJET2.1 the CloneJET ${ }^{\mathrm{TM}}$ PCR Cloning Kit (Fermentas, St. Leon-Rot) was used according to manufacturer's instructions. 
Digested and purified CDX1/CDX2 promoter fragments and pGL2Basic vectors were ligated using the Rapid DNA Ligation Kit (Fermentas, St. Leon-Rot). $100 \mathrm{ng}$ vector DNA and $200 \mathrm{ng}$ promoter fragments were incubated with $1 \mu \mathrm{l}$ T4 Ligase in $1 \times$ Rapid ligation buffer for $10 \mathrm{~min}$ at $22^{\circ} \mathrm{C}$.

The resulting ligation reaction was used for transformation of competent $E$. coli (3.6.2).

\subsubsection{Microarray analysis}

Biological triplicates of HCT116, SW480 and MCF7 cells were transfected with 2 specific siRNAs for ß-catenin, BCL9 and BCL9-2 knockdown and 4 unspecific non-targeting siRNAs as controls, with a final concentration of $50 \mathrm{nmol}$ for $48 \mathrm{~h}$ according to standard protocols. Extraction of RNA was performed using TRI Reagent (Ambion, Darmstadt) according to manufacturer's instructions (3.7.1.2).

cDNA synthesis, in vitro transcription, hybridization and initial analyses of expression data were performed in the "Transkritomanalyselabor" (TAL), Göttingen. cDNA synthesis and in vitro transcription (IVT) were performed according to the manufacturer's recommendation using "Low RNA Input linear Amplification Kit Plus, One Color" protocol (Agilent Technologies, Böblingen) and the RNA Spike-In Kit for One color (Agilent Technologies, Böblingen). Global gene expression analysis was done using the Human $4 \times 44 \mathrm{~K}$ design array 026652 (Agilent Technologies, Böblingen). Hybridizations were performed for 17 hours at $10 \mathrm{rpm}$ and $65^{\circ} \mathrm{C}$ in the Hybridization Oven (Agilent Technologies, Böblingen). Cy3 intensities were detected by one-color scanning using an Agilent DNA microarray scanner at 5 micron resolution. Intensity data were extracted using Agilent's Feature Extraction (FE) software (version 10.7.3.1) including a quality control based on internal controls using Agilent's protocol GE1_107_Sep09. All chips passed the quality control and were analyzed using the Limma package (1) of Bioconductor (2). 


\subsection{Biochemistry}

\subsubsection{Isolation of proteins from cell cultures}

\subsubsection{Isolation of whole cell protein lysates for SDS-polyacrylamide gel electrophoresis (SDS-PAGE)}

Whole cell protein lysates were obtained from confluent cell cultures grown in $10 \mathrm{~cm}$ culture dishes. After 2 washing steps with ice-cold $1 \times$ PBS, cells were lysed with $1 \times$ RIPA buffer supplemented with protease inhibitors, harvested with a cell scraper and transferred into a new reaction tube. Lysates were centrifuged with maximum speed at $4^{\circ} \mathrm{C}$ after incubation on ice for $10 \mathrm{~min}$. The supernatant was transferred into a new reaction tube and stored at $-80^{\circ} \mathrm{C}$ or immediately used for SDS-PAGE.

Protein concentrations were determined according to Bradford standard protocol (Bradford, 1976). To the appropriate amount of protein, $4 \times$ Laemmli probe puffer (LPP) was added. It was thenboiled for $5 \mathrm{~min}$ at $95^{\circ} \mathrm{C}$ and stored at $-20^{\circ} \mathrm{C}$.

\subsubsection{Isolation of nuclear and cytoplasmatic proteins for SDS-PAGE}

To separate nuclear and cytoplasmatic protein fractions, confluent cell cultures grown in 6 well culture plates were immediately lysed in $100 \mu$ l of buffer A supplemented with protease inhibitors, scabbed off and transferred into a new reaction tube. After 15 min incubation on ice $5 \mu \mathrm{l}$ of $10 \%$ NP40 were added. The samples were vortexed for $20 \mathrm{~s}$ and centrifuged for $2 \mathrm{~min}$ at $3000 \mathrm{rpm}$ at $4^{\circ} \mathrm{C}$. An appropriate amount of $4 \times$ LPP was added to the supernatant containing the cytoplasmatic fraction. The remaining nuclear pellet was resuspended in $60 \mu \mathrm{l}$ ice-cold buffer $\mathrm{C}$ (supplemented with protease inhibitors) and incubated for $15 \mathrm{~min}$ at $4^{\circ} \mathrm{C}$ while rocking. After centrifugation for $5 \mathrm{~min}$ at $3000 \mathrm{rpm} 4^{\circ} \mathrm{C}$ the supernatant was supplemented with an appropriate amount of $4 \times$ LPP. All samples were boiled for $5 \mathrm{~min}$ at $95^{\circ} \mathrm{C}$ and stored at $-20^{\circ} \mathrm{C}$.

\subsubsection{Isolation of protein lysates for immunoprecipitation}

Proteins for immunoprecipitations were isolated according to 2.8.1.2 and 2.8.1.3. For generation of whole cell lysates, in contrast, MS buffer was used to lyse the cells. For generation of nuclear lysates $10 \mathrm{~cm}$ culture dishes containing confluent cells were lysed with $500 \mu \mathrm{l}$ buffer A. Finally, solubilisation of nuclear pellets was achieved with $100 \mu \mathrm{l}$ buffer A. For immunoprecipitation $400 \mu \mathrm{l}$ MS buffer was added to the nuclear protein lysate. 
$75 \mu \mathrm{l}$ of cell lysates served as INPUT-control. To these, $25 \mu \mathrm{l} 4 \times$ Laemmli probe buffer (LPP) was added. They were stored at $-20^{\circ} \mathrm{C}$.

$\begin{array}{ll}\text { MS buffer } & 20 \mathrm{mM} \text { Tris pH7.4, } 150 \mathrm{mM} \mathrm{NaCl}, 1 \% \text { NP-40, 0.5\% Sodium Deoxychelate, } \\ & \text { 1mM EDTA, 0.1\% SDS , 20 mM NaF, Protease Inhibitor Cocktail (Roche) } \\ \text { MSW buffer } & 50 \mathrm{mM} \text { Tris pH7.4, 150 mM NaCl, } 20 \mathrm{mM} \mathrm{NaF} \\ \text { Buffer A } & 10 \mathrm{mM} \text { Hepes, pH 7.9, } 10 \mathrm{mM} \mathrm{KCl}, 0.1 \mathrm{mM} \text { EDTA, } 0.1 \mathrm{mM} \text { EGTA } \\ \text { Buffer C } & 20 \mathrm{mM} \text { Hepes, pH 7.9, } 400 \mathrm{mM} \mathrm{NaCl}, 1 \mathrm{mM} \text { EDTA, } 1 \text { mM EGTA }\end{array}$

\subsubsection{Isolation of proteins for Luciferase assays}

Whole cell lysates for Luciferase assays were obtained from cells previously transfected with Luciferase-reporters as described in 3.9.3.2.

For isolation of Luciferase proteins the medium was removed and $50 \mu \mathrm{l}$ of $1 \times$ passive lysis buffer (Qiagen, Hilden) was added to the confluent cells. The reaction was incubated for 60 $\min$ at RT. Cell lysates were transferred into reaction tubes and centrifuged for 2 min with maximum speed. Measurement of Luciferase activity was performed as described in 3.8.4.

\subsubsection{SDS-PAGE and Western Blot}

Protein samples from whole cell lysates or nuclear/cytoplasmatic fractions were used for SDSPAGE and subsequent Western blotting to determine the expression of specific proteins in cell cultures per se or after treatment with siRNAs. The molecular weight of the separated proteins was determined by comparison with a pre-stained protein ladder.

Proteins were concentrated in the stacking gel at $100 \mathrm{~V}$, followed by separation at 150 to 200 V. Subsequently, proteins were transferred onto methanol-activated Hybond-P PVDF membranes at $4^{\circ} \mathrm{C}$ using a wet blot system. Depending on the size of proteins $0.5 \mathrm{~mA}$ were applied for $1.25 \mathrm{~h}$ to $1.5 \mathrm{~h}$. For immunostaining the membranes were previously blocked with blocking solution to avoid unspecific binding of the primary antibody (Table 6) which was incubated $\mathrm{o} / \mathrm{n}$ at $4^{\circ} \mathrm{C}$ with dilution according to the instructions of the manufacturer. After the membranes were washed 3 times with TBST, the horseradish peroxidase (HRP) coupled secondary antibody diluted in TBST with $5 \%$ powdered milk was added. The membranes were then incubated for $45 \mathrm{~min}$ at RT. The membranes were again washed 3 times with TBST. 
Proteins bound to antibody-complexes were visualized after incubation with 1:1 (v/v) ECL solution $A$ and $B$ for $60 \mathrm{~s}$. The resulting chemo-luminescent signals were detected with LAS4000 imaging system. Finally, the membranes were washed with TBST to remove ECL solution residues and air-dried for storage at $4^{\circ} \mathrm{C}$.

SDS transfer buffer $\quad 2.5 \mathrm{mM}$ Tris, $19.2 \mathrm{mM}$ Glycine, 0.01\% (w/v) SDS

Stacking gel $\quad 12 \%$ Acrylamid/Bis (10\%), $25 \%(v / v)$ Stacking buffer, $0,01 \%(v / v)$ TEMED, $0,1 \%(v / v)$ APS

Separating gel $33 \%$ Acrylamid/Bis (30\%), $25 \%$ Separating buffer, $0.01 \%$ (v/v)

Separating gel TEMED, $0.1 \%$ (v/v) APS (10\%)

TBST 10\% Acrylamide/Bis, $2.5 \mathrm{ml}$ Separating Buffer (4 x), $5 \mathrm{ml}$ water, $100 \mu \mathrm{l}$ APS (10\%), $10 \mu$ I TEMED

$50 \mathrm{mM}$ Tris- $\mathrm{HCl}$ (pH 7.4), $150 \mathrm{mM} \mathrm{NaCl}, 0.05 \%$ (v/v) Tween 20

Blocking Solution

5\% powdered milk in TBST

ECL solution A

$2.5 \mathrm{mM}$ Luminol, $0.4 \mathrm{mM}$ p-coumaric acid, 0.1 M Tris- $\mathrm{HCl}(\mathrm{pH} 8.5)$

ECL solution $B$ $0.05 \%(v / v), 35 \% \mathrm{H}_{2} \mathrm{O}_{2}, 0.1 \mathrm{M}$ Tris- $\mathrm{HCl}(\mathrm{pH} 8.5)$

Stacking buffer

$0.5 \mathrm{M}$ Tris, $0.4 \%(\mathrm{w} / \mathrm{v})$ SDS

(pH 6.8)

Separating buffer $\quad 1.5 \mathrm{M}$ Tris, $0.4 \%(\mathrm{w} / \mathrm{v})$ SDS

(pH 8.8)

\subsubsection{Immunoprecipitation}

Protein lysates used to identify specific protein-protein-complexes were obtained according to 3.8.1.3.

To couple specific proteins to sepharose beads $2 \mu \mathrm{g}$ of respective antibodies were added to the cell lysates and incubated for $30 \mathrm{~min}$ at $4^{\circ} \mathrm{C}$ followed by addition of $100 \mu \mathrm{l}$ pre-swollen Asepharose beads ( $50 \%$ slurry) in MS buffer. The protein-antibody-bead-complexes were generated during incubation of the sample $\mathrm{o} / \mathrm{n}$ at $4^{\circ} \mathrm{C}$ rotating. Loaded beads were pelleted and centrifuged with $2000 \mathrm{rpm}$ at $4^{\circ} \mathrm{C}$. After washing the complexes 6 times with MS wash buffer the pellets were resuspended in $40 \mu \mathrm{l}$ MS buffer and directly used for massspectrometric analyses. For Western Blot analyses $25 \mu \mathrm{l} 4 \times$ Laemmli probe buffer (LPP) was added. Samples were stored at $-20^{\circ} \mathrm{C}$. 


\subsubsection{Luciferase assays}

Luciferase assays were performed in duplicates according to the protocols published by Hampf (Hampf and Gossen, 2006).

$40 \mu \mathrm{l}$ of the supernatant of lysed cells (as described in 2.8.1.4) was transferred into 96-well luminometer plates. Luminescence intensity was measured with the Berthold Multimode Reader TriStar LB 946. Injectors were washed before and after usage with 20 cycles of $\mathrm{H}_{2} \mathrm{O}, 20$ cycles of ethanol, 20 cycles of $\mathrm{H}_{2} \mathrm{O}$ and 20 cycles of air.

Firefly Luciferase activity was determined by injecting $100 \mu \mathrm{l}$ P/Fluc-A solution, followed by 0.5 $\mathrm{s}$ delay and $10 \mathrm{~s}$ measurement of luminescence. Accordingly, Renilla Luciferase activity was quantified by injecting $75 \mu \mathrm{P} /$ Rluc-B solution followed by $0.5 \mathrm{~s}$ delay and $10 \mathrm{~s}$ measurement of luminescence. Firefly Luciferase activity was normalized to the respective Renilla Luciferase activity. In addition, Luciferase (LUC)-promoter-reporter activities were relativized to their empty control LUC-reporters. Mean values of at least 3 independent experiments and their standard deviations were calculated and displayed graphically using Microsoft Excel. Statistical significances were based on conventional students t-tests.

P/Fluc-A solution $15 \mathrm{mM} \mathrm{MgSO}_{4}, 0.1 \mathrm{mM}$ EDTA, $25 \mathrm{mM}$ DTT, $1 \mathrm{mM}$ ATP, $200 \mu \mathrm{M}$ coenzyme A, (pH 8.0) $200 \mu \mathrm{M}$ luciferin, $200 \mathrm{mM}$ Tris-HCl

P/Rluc-B solution $\quad 0.05 \%(v / v), 35 \% \mathrm{H}_{2} \mathrm{O} 2,0.1 \mathrm{M}$ Tris- $\mathrm{HCl}(\mathrm{pH}$ 8.5) $10 \mathrm{mM} \mathrm{NaAc}, 15 \mathrm{mM}$ EDTA, (pH 5.0) $\quad 500 \mathrm{mM} \mathrm{NaCl}, 50 \mu \mathrm{M}$ phenyl-benzothiazole, $4 \mu \mathrm{M}$ benzyl-coelenterazine, $500 \mathrm{mM} \mathrm{Na}_{2} \mathrm{SO}_{4}, 25 \mathrm{mM} \mathrm{Na} 4 \mathrm{PP}_{\mathrm{i}}$

\subsection{In vivo model systems}

\subsubsection{Mouse model systems}

To investigate the role of BCL9-2 in vivo a mouse model has been established (Brembeck et al., 2011). To this end the 2.1-kilobase mouse keratin 19 promoter (Brembeck et al., 2001) was linked to a rabbit ß-globin intron and the flag tagged, full-length CDNA of mouse BCL9-2 and a BGH-polyA. Genotypes of K19-BCL9-2 transgenic offspring were determined by conventional PCR (as described in 2.7.3.1) using specific oligonucleotides targeting the BCL9-2 transgene (Table 12). 
Transgenic K19-BCL9-2 mice were bred with $\mathrm{APC}^{\mathrm{Min} /+}$ mice (Table 15) to investigate the influence of transgenic BCL9-2 expression on tumor formation. $\mathrm{APC}^{\mathrm{Min} /{ }^{+}} ; \mathrm{K} 19-\mathrm{BCL9}-2$ mice were followed up to 5.5 month of age or until mice showed severe tumor-related diseases before they were dissected. Macroscopic tumors of the intestine $\left(\geq 0.5 \mathrm{~mm}^{2}\right)$ were identified with a dissection lens. The number and size of adenomas was determined in the small intestine and the colon. To secure accurate analyses counted numbers of adenomas were regularly rechecked by additional counting and measuring by a second person. Box Plot analyses were evaluated and graphically visualized by SPSS 19.0. Significances were calculated using the Whitney-U test. The significance for survival analysis was defined according to P log-rank test.

To label proliferating cells in the intestine $0.1 \mathrm{mg} / \mathrm{g}$ of bodyweight bromo-deoxyuridine in $1 \mathrm{x}$ PBS (Table 3 ) was injected intraperitoneally $2 \mathrm{~h}$ before the mice were dissected. Mice were raised by the ENI animal facility, Göttingen with the approval by governmental authorities.

\subsubsection{Tissue processing for immunohistochemistry and Immunofluorescence}

Tissue of transgenic and control mice were fixed in $4 \%$ paraformaldehyde in $1 \times$ PBS at $4^{\circ} \mathrm{C}$ overnight and washed with cold $1 x$ PBS. For long-term storage, tissue samples were transferred into $70 \%$ ethanol or dehydrated according to standard protocols (75\% Ethanol, 80\% Ethanol, 90\% Ethanol, 96\% Ethanol and 2 times 100\% Ethanol for $1.5 \mathrm{~h}$ each). For $3 \mu \mathrm{m}$ serial sections samples were incubation in xylol 2 times $1.5 \mathrm{~h}$ and paraffin for $1.5 \mathrm{~h}$ to $12 \mathrm{~h}$ and stored at RT or processed according to 3.3.5.

\subsubsection{Immunostaining of tissues}

For Hematoxylin and Eosin (H\&E) staining and immunostaining $3 \mu \mathrm{m}$ serial tissue sections (3.9.2) from transgenic and control mice were rehydrated. Tissue sections were incubated 3 times for $5 \mathrm{~min}$ in Xylol, for $3 \mathrm{~min}$ in $100 \%, 96 \%, 80 \%$ and $70 \%$ ethanol each and finally washed in $\mathrm{dH}_{2} \mathrm{O}$.

\subsubsection{Hematoxylin and Eosin staining (H\&E)}

De-waxed and rehydrated tissue sections were incubated in Hematoxylin for 2 min and rinsed with $\mathrm{dH}_{2} \mathrm{O}$ for 5-10 min followed by 2 min incubation in Eosin. Hematoxylin stained sections were dehydrated in a rising ethanol-series (70\% for $10 \mathrm{sec}, 80 \%$ for $10 \mathrm{sec}, 96 \%$ for $3 \mathrm{~min}$ and 
twice in $100 \%$ for $3 \mathrm{~min}$ ), followed by 3 times incubation in xylol for $3 \mathrm{~min}$. They were finally mounted with Rotiø-Histokitt (Roth, Karlsruhe).

\subsubsection{Immunohistochemistry}

The expression of proteins was examined by staining of de-waxed and rehydrated tissue sections with the corresponding specific antibodies (Table 7). To this end, samples were boiled in pre-heated Antigene retrieval buffer for $20 \mathrm{~min}$ and cooled down to RT in a water bath. The sections were incubated with $1 \% \mathrm{H}_{2} \mathrm{O}_{2}$ for $10 \mathrm{~min}$ at $\mathrm{RT}$ and then washed once in $\mathrm{dH}_{2} \mathrm{O}$ for 5 min and twice in $1 \times$ PBS for 5 min each. Endogenous peroxidases were blocked by incubation in IHC blocking solution for $30 \mathrm{~min}$ at RT. Tissue sections were incubated $\mathrm{o} / \mathrm{n}$ at $4^{\circ} \mathrm{C}$ with the specific primary antibody diluted in IHC blocking solution in a humidified atmosphere. After washing for 3 times $5 \mathrm{~min}$ in 1 x PBS the samples were incubated for 30 to $45 \mathrm{~min}$ at RT with the secondary HRP-conjugated antibody (Dako EnVision Kit, Dako, Hamburg) and then washed 3 times in 1 x PBS for 5 min. Antibody complexes were detected according to manufacturer's instructions. For counter-staining, sections were incubated for $10 \mathrm{sec}$ with Hematoxylin and 5 to 10 min rinsed in $\mathrm{dH}_{2} \mathrm{O}$. For imaging, stained sections were dehydrated and mounted with Roti ${ }^{\circledR}$-Histokitt (Roth, Karlsruhe).

Antigene retrieval buffer $10 \mathrm{mM}$ Tris, $1 \mathrm{mM}$ EDTA, $\mathrm{pH} 9.0$

IHC Blocking Buffer $\quad 10 \%$ rabbit serum, $1 \%$ BSA in 1x PBS

\subsubsection{Immunofluorescence staining on paraffin embedded tissues}

Protein expression, corresponding specific antibodies (Table 10) and the indirect fluorescent staining were used. The paraffin sections were rehydrated by incubation in xylol ( $3 \times 5 \mathrm{~min})$, ethanol $\left(100 \%, 96 \%, 80 \%\right.$ and $70 \%$ for 3 min each step) and then washed in $\mathrm{dH}_{2} \mathrm{O}$. Following rehydration, the antigene retrieval was performed by boiling the samples in preheated Antigene retrieval buffer (Table 5) for $20 \mathrm{~min}$. The samples were cooled down to RT in a cold water bath. After 30 min (RT) blocking in AB buffer (Table 5), the sections were incubated over-night $\left(4^{\circ} \mathrm{C}\right)$ with the specific primary antibody (diluted in blocking solution) in a humidified atmosphere. After washing ( $3 \times 20 \mathrm{~min}$ in PBST) the samples were incubated for 30 to $45 \mathrm{~min}$ ( $R T$, humidified atmosphere) with the secondary fluorophore-conjugated antibody (diluted in $A B$ buffer) and then washed again in PBST $(3 \times 20 \mathrm{~min})$. The counter-staining with DAPI was performed in the penultimate washing step by diluting DAPI stock solution to $0.1 \mu \mathrm{g} / \mathrm{ml}$ in 
PBST. For microscopy the sections were mounted in Immu-Mount (ThermoScientific, Bonn) and stored at $4^{\circ} \mathrm{C}$ in the dark.
AB buffer
$0.1 \%$ Tween, $10 \%$ horse serum in $1 \times$ PBS
PBST
$1 \times$ PBS supplemented with $0.05 \%(v / v)$ Tween
Blocking Solution
$1 \times$ PBS supplemented with $0.5 \%(v / v)$ Triton X-100, $0.5 \%(w / v)$ BSA

\subsubsection{Scoring of human tissue microarray}

Colorectal cancer tissues were obtained from the Tumor Bank of the Department of Surgical Oncology, Charité Medical School Berlin. All patients underwent surgery followed up for a minimum of three years. Specimens were transferred to a paraffin array block and $1 \mu \mathrm{m}$ tissue sections subjected to immunohistochemistry. B-catenin, BCL9 and BCL9-2 stainings were scored by three independent investigators using the expression score according to (Sinicrope et al., 1995). The immunoreactive score (0 to 9) was calculated as the product of the staining intensity and the percentage of positive epithelial cells. The staining intensity was defined as 0 (negative), 1 (low), 2 (moderate) and 3 (strong).The percentage of positive epithelial cells was scored as $0(0 \%), 1(<30 \%), 2(30 \%-60 \%)$ and $3(>60 \%)$. Statistics were evaluated using the Mann-Whitney-U test.

\subsubsection{Cell culture model systems}

\subsubsection{Cultivation and storage of cell cultures}

Cell cultures were stored in $1 \times$ culture/trasnfection medium supplemented with $10 \%$ DMSO in cryo tubes. To this end, cells were slowly frozen $\mathrm{o} / \mathrm{n}$ at $-80^{\circ} \mathrm{C}$ in freezing boxes followed by long-time storage at $-150^{\circ} \mathrm{C}$. For cultivation, cells were thawed in a water bath at $37{ }^{\circ} \mathrm{C}$ and transferred into $10 \mathrm{ml} 1 \times$ PBS. After centrifugation at $1000 \mathrm{rpm}$ for $3 \mathrm{~min}$, cell-pellets were resuspended in culture medium, supplemented with 10\% FCS as recommended by the suppliers and transferred into a $75 \mathrm{~cm}^{2}$ or $175 \mathrm{~cm}^{2}$ flasks and cultivated at $37^{\circ} \mathrm{C}$ in a $5 \% \mathrm{CO}_{2}$ / $95 \%$ humidity atmosphere. Depending on their confluence cells were split 1:5 to 1:10 every 3 to 5 days. Therefore, cells were detached with $1 \mathrm{ml}$ trypsin after washing with $1 \times$ PBS. Detached cells were resuspended in culture medium and spread into new culture flasks. 


\subsubsection{Transfection}

Cell lines were transfected with siRNA (Table 13) or endotoxin-free, highly pure plasmid DNA (Table 16) using Lipofectamine2000 (Invitrogen, Darmstadt) according to manufacturer's instructions. $100 \mathrm{ng}$ TOP/FOP-, -136 bp CyclinD1- and -5600 bp Axin2-reporters or $200 \mathrm{ng}$ of CDX1 and CDX2 promoter LUC-reporters were used for transfection. In addition, 25 ng Renilla Luciferase was added to normalize Luciferase-reporters activities.

Cells were seeded $12 \mathrm{~h}-16 \mathrm{~h}$ prior to transfection. The used cell numbers of different cell lines were summarized in Table 18.

Table 18: Cells / $\mathrm{ml}$ used for transfection with siRNA and with overexpression-plasmids (oe)

\begin{tabular}{l|l|l|l} 
cell line & $\begin{array}{l}\text { cells/ml (siRNA) } \\
\text { in } \mathbf{2 4} \text { well plates }\end{array}$ & $\begin{array}{l}\text { cells/ml (siRNA) } \\
\text { in } \mathbf{6} \text { well plates }\end{array}$ & $\begin{array}{l}\text { cells } / \mathbf{m l} \text { (oe) } \\
\text { in } \mathbf{1 0} \mathbf{~ c m} \text { dishes }\end{array}$ \\
\hline \hline HEK293 & $1.875 \times 10^{5}$ & & $1.25 \times 10^{5}$ \\
\hline SW480 & $1.875 \times 10^{5}$ & $1.25 \times 10^{5}$ & \\
\hline DLD1 & $1.25 \times 10^{5}$ & $1.25 \times 10^{5}$ & \\
\hline HCT116 & $1.875 \times 10^{5}$ & $1.67 \times 10^{5}$ & \\
\hline SW48 & & &
\end{tabular}

For over-expression of proteins in vivo in addition to Luciferase-reporters (LUC-reporters) or for protein isolation, cells were transfected with transfection mixes according to Table 19. Transfection mix A including OptiMEM and siRNA/plasmids and Transfection mix B including OptiMEM and Lipofectamine2000 were incubated for 5 min before they were mixed and further incubated for 20 min at RT. Consequently, the mix was added to the cells. In analytical approaches, the plates were centrifuged for $10 \mathrm{~min}$ at $300 \mathrm{rpm}$ to increase the transfection efficiency.

After siRNA transfection cells were incubated for $48 \mathrm{~h}$ followed by transfection of LUCreporters and further incubation of $36 \mathrm{~h}$. For overexpression with and without LUC-reporter transfection cells were incubated for $48 \mathrm{~h}$. Cells were harvested according to 3.8.4. 
Table 19: Components of Lipofectamine based transfection mixes

\begin{tabular}{l|l|c|l} 
detergents & in $\mathbf{2 4}$ well plates & in $\mathbf{6}$ well plates & in $\mathbf{1 0 ~ c m ~ d i s h e s ~}$ \\
\hline \hline $\begin{array}{l}\text { OptiMEM Mix A and B } \\
\text { Lipofectamine2000 }\end{array}$ & $0.5 \mu \mathrm{l}$ each/well & $250 \mu \mathrm{l}$ each/well & $2,5 \mathrm{ml}$ each/well \\
\hline $\begin{array}{l}\text { Overexpression and } \\
\text { LUC-Firefly plasmids }\end{array}$ & $100-200 \mathrm{ng}$ & $\mathrm{X}$ & $15 \mu \mathrm{l}$ \\
\hline $\begin{array}{l}\text { LUC-Renilla plasmid } \\
\text { Lipofectamine2000 }\end{array}$ & $25 \mathrm{ng}$ & $\mathrm{Xl}$ & $15-20 \mu \mathrm{g}$ \\
\hline siRNA & $1 \mu \mathrm{l}(25 \mathrm{pmol}$ each $)$ & $5 \mu \mathrm{l}(25 \mathrm{pmol}$ each $)$ & $\mathrm{X}$
\end{tabular}

\subsection{Software}

Table 20: Software

\begin{tabular}{l|l} 
software & developer/manufacturer \\
\hline \hline Microsoft Office 2010 & Microsoft \\
\hline GENtle & University of Cologne, Germany \\
\hline SPSS 19.0 & SPSS Inc. \\
\hline $\begin{array}{l}\text { Transfac } \\
\text { (MATCH/PATCH) }\end{array}$ & Transfac Professional 2008.4 (Kel et al., 2003; Matys et al., 2003) \\
\hline T-COFFEE ${ }^{\circledR}$ & T-Coffee Home Page (Di et al., 2011; Notredame et al., 2000) \\
\hline Scaffold3 & Proteome Software, Inc. \\
\hline Leica Application Suite & Leica
\end{tabular}

\subsection{Statistics}

Statistical significances of qPCR and Luciferase-reporters gene assays were calculated by the conventional two sided t-test using Microsoft Excel. Significances of adenoma development were calculated by $\mathrm{P}$ log-rank test, significances of nuclear expression scores by the MannWhitney U Test using SPSS 19.0 software. 


\section{Results}

\subsection{BCL9 and BCL9-2 expression in normal intestine and during intestinal tumorigenesis}

So far, the expression of the Wnt/ß-catenin co-factors BCL9 and BCL9-2 normal intestinal epithelia and tumors of mice and humans were not characterized. Recent studies described that BCL9 proteins are deregulated in different tumor entities (Brembeck et al., 2004;Sakamoto et al., 2007; Toya et al., 2007). However, detailed investigations were missing.

\subsubsection{BCL9-2 expression is restricted to the villi of the normal intestine and is up- regulated in early stages of intestinal tumorigenesis}

To get an insight into the distribution and expression of the BCL9 proteins, intestinal tissue sections from late embryonic and adult mice were subjected to immunohistochemistry (Figure 10A). Antibodies were generated that specifically recognize BCL9 and BCL9-2 and B-catenin (Brembeck et al., 2011). In addition, the expression of the proteins in adenomas from APC ${ }^{\mathrm{MIN} /+}$ mutant mice was determined (Figure 10). Immunohistochemical stainings of tissue microarrays from human normal mucosa and adenomas were scored for the nuclear expression of the three proteins (Figure 10C).

In the intestines of embryonic and adult mice, nuclear BCL9 was strongly expressed in all mesenchymal and epithelial cells in the crypts and villi the intestine (Figure 10A, left panel). In contrast, BCL9-2 expression was restricted to the villi of late embryonic and adult intestines (Figure 10A, right panel, black arrowheads) but was absent in the crypts of the small intestine and in the colon (Figure 10A, right panel, white arrowhead), where ß-catenin/Wnt-signaling is active. Adenomas of $\mathrm{APC}^{\mathrm{MIN} /+}$ mice were highly proliferative as indicated by BrdU staining. Increased nuclear ß-catenin expression was determined suggesting actived Wnt/ß-catenin signaling in adenomas (Figure $10 \mathrm{~B}$ and C). Interestingly, adenomas of $\mathrm{APC}^{\mathrm{MIN} /+}$ mice and human specimen also exhibited increased BCL9-2 expression (Figure 10B, black arrow) compared to adjacent crypts of the normal mucosa (Figure 10C). In contrast, BCL9 protein expression remained unchanged in adenomas of $\mathrm{APC}^{\mathrm{MIN} /+}$ mice and human specimen (Figure $10 \mathrm{~B}$ and $\mathrm{C}$ ). 
A. normal intestine

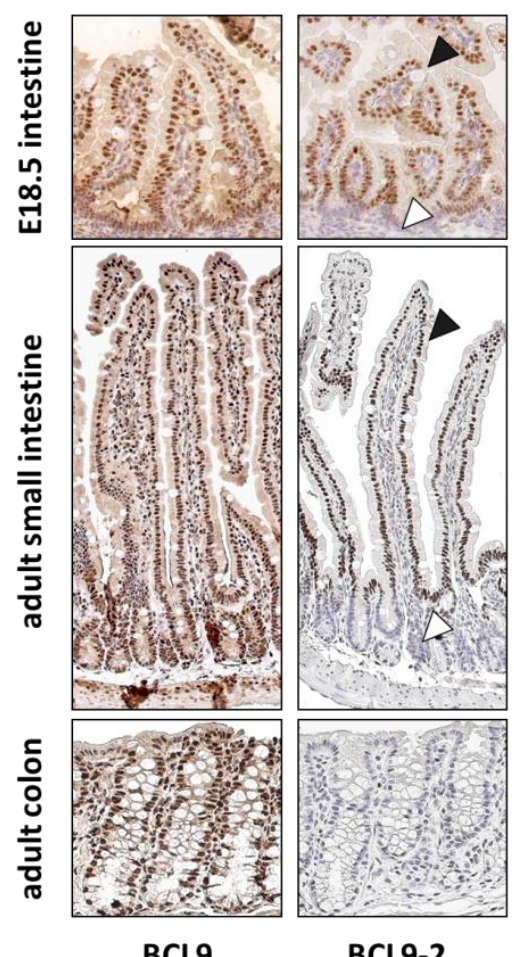

BCL9

BCL9-2

\section{B. adenoma formation}

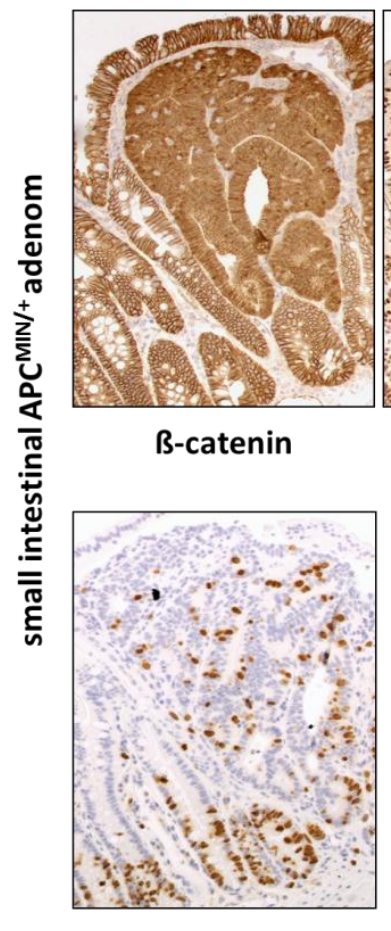

BrdU

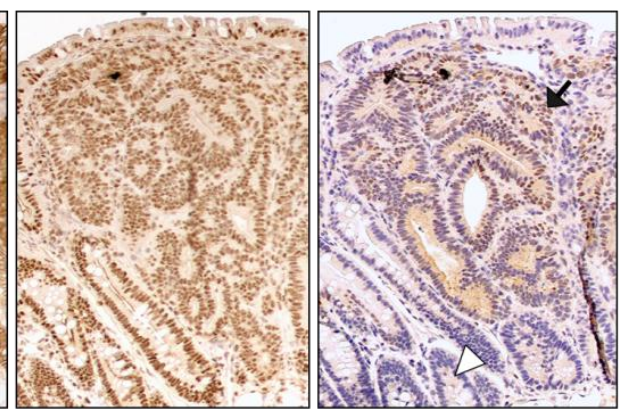

BCL9

BCL9-2

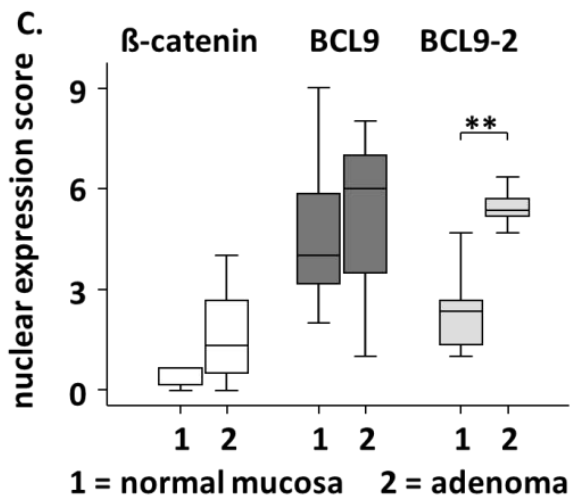

Figure 10: Expression of BCL9 and BCL9-2 in normal embryonic and adult intestines and in adenomas Immunohistochemistry on serial sections of $(A)$ normal intestines of late embryonic and adult mice and (B) a representative adenoma of an $\mathrm{APC}^{\mathrm{MIN} /+}$ mouse. Specific antibodies targeting the indicated proteins were used. (C) nuclear expression score of $\beta$-catenin, BCL9 and BCL9-2 obtained from human normal mucosa and adenomas ( $\left.{ }^{*} p \leq 0.05\right)$. (Brembeck, Wiese et al., 2011)

\subsubsection{BCL9-2, but not BCL9, is highly expressed in human colon cancers}

The distribution and expression of B-catenin, BCL9 and BCL9-2 was further investigated in different stages during colon carcinogenesis. Therefore a human tissue microarray of paired samples from 105 patients was established containing normal mucosa, adjacent transitional mucosa and colorectal cancers without (stage I, $n=58$ ) or with metastases (stage III/IV, $n=47$ ). The nuclear expression score was defined as the product of the presence of immunopositive cells and intensity of the staining.

Highly significant increased expression of BCL9-2 was found in more than $90 \%$ of human colon tumors. Comparison of the nuclear expression score of stage I (without metastasis) and stage II/IV (with metastasis) cancers revealed that BCL9-2 was strongly expressed in all cancers, independently of the presence of metastasis (Figure $11 \mathrm{~A}$ and B). Strong ß-catenin expression was only found in approximately $60 \%$ of the tumors (Figure $11 \mathrm{~A}$ ). In contrast, no significant changes were detected for the BCL9 protein (Figure 11B). 
A.
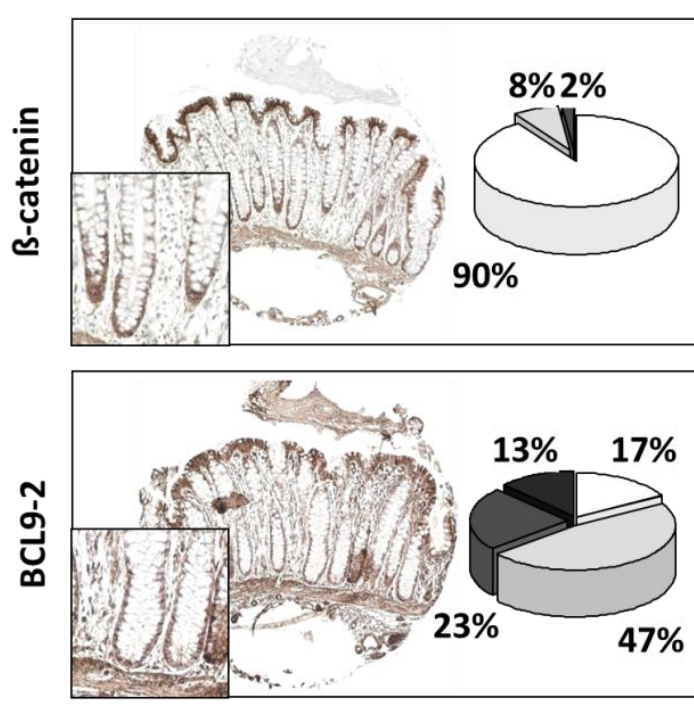

absent/low (0-2) moderate (>2-4)
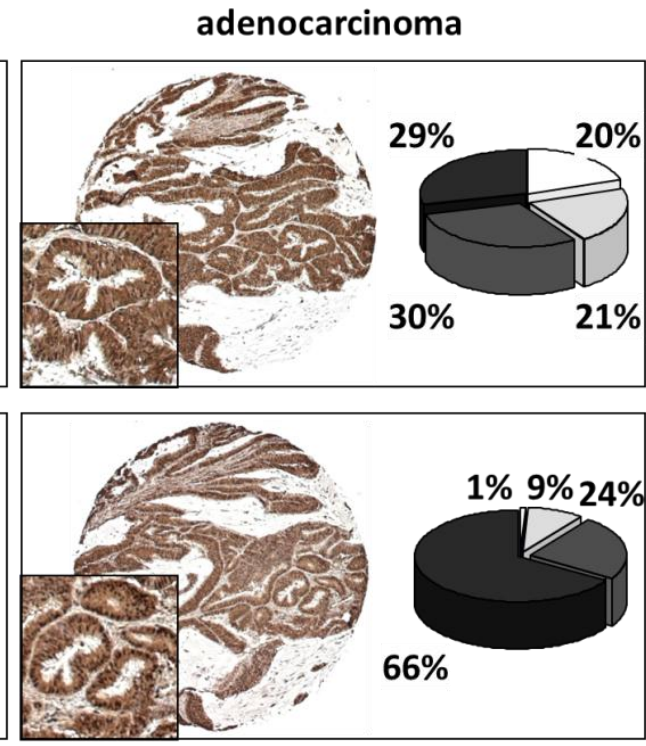

elevated (>4-6)

strong (>6-9)
B.

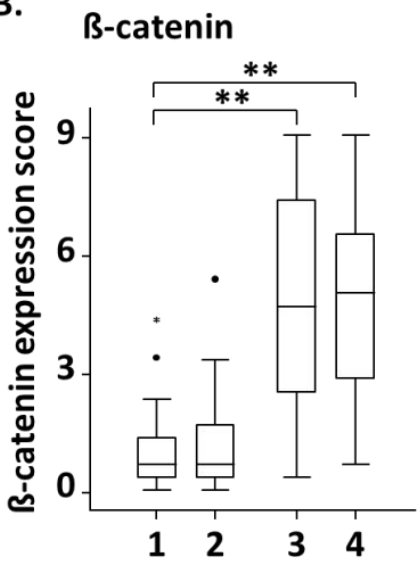

BCL9

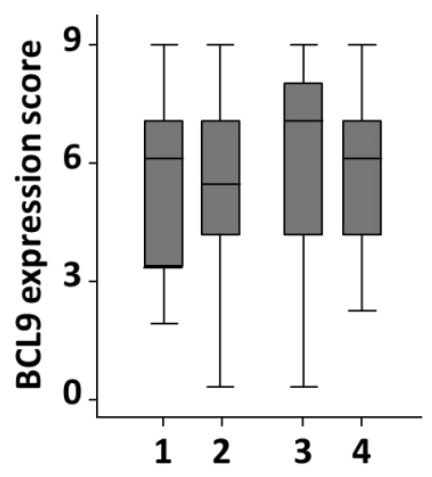

BCL9-2

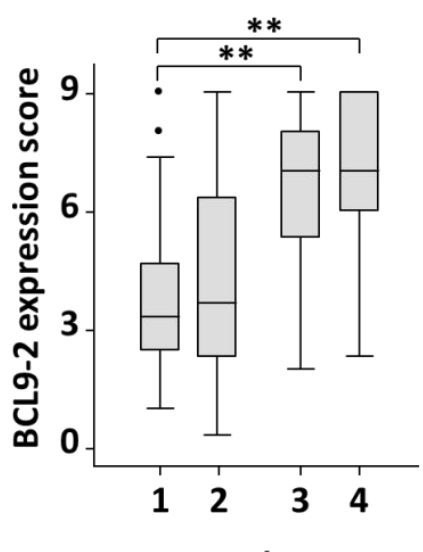

\section{1-normal mucosa 2-transition mucosa 3-stage I cancer 4-stage III/IV cancer}

\section{Figure 11: BCL9-2 is up-regulated in human adenocarcinomas}

Immunohistochemistry and scoring of human tissue microarrays of matched specimens from patients with stage I, III and IV colon cancer (A) Immunostainings depict the protein expression of B-catenin and BCL9-2 on matched, representative tumor samples. Pie charts show the relative frequency of the immunoreactive score for BCL9-2 and B-catenin expression in adenocarcinomas and normal mucosa, (B) Boxplot analyses of the nuclear expression score of $B$-catenin, BCL9 and BCL9-2 in normal and transient mucosa, non-metastatic(stage I) and metastatic cancer (stage II/IV) ( $\mathrm{p}<0.01)$. (Brembeck, Wiese et al., 2011)

Thus, intestinal BCL9-2 expression is restricted to the villi and not present in the crypts, where Wnt-signaling is active. Moreover, up-regulation of BCL9-2 protein expression occurs early during intestinal tumorigenesis, culminating in $90 \%$ of highly BCL9-2 positive human adenocarcinomas. In contrast, BCL9 expression is not changed during early and even late stages of intestinal tumorigenesis compared to normal mucosa. These data indicate that BCL9- 
2 might contribute to tumor progression already in early stages of intestinal tumorigenesis. Further, BCL9-2 might be a useful marker, which indicates tumor progression.

\subsection{BCL9-2 overexpression promotes intestinal tumor development}

\subsection{1 $\mathrm{BCL9-2}$ expression in compound $\mathrm{APC}^{\mathrm{Min} /+}$; K19-BCL9-2 transgenic mice}

To investigate the tumorigenic potential of BCL9-2 in vivo, a transgenic mouse model was analyzed (Brembeck et al., 2011). In this model, BCL9-2 overexpression was induced by a K19 promoter which is active in simple epithelia, including the intestine (Brembeck et al., 2000). Ectopic expression of $\mathrm{BCL} 9-2$ on a pure $\mathrm{C57BL} / 6$ background led to infrequent, undifferentiated intestinal tumor formation in aged mice (>15 month)(Brembeck et al., 2011). To induce tumorigenesis K19-BCL9-2 transgenic mice were further crossed with C57BL/6$\mathrm{APC}^{\mathrm{Min} /+}$ - mice, which displayed spontaneous development of adenomas in the small intestine and colon (Su et al., 1992). The specific expression of ectopic BCL9-2 in compound transgenic mice was verified RT-PCR (Figure 12A) and immunohistochemistry (Figure 12B).

Endogenous BCL9-2 protein was expressed in adenomas of $A P C^{\mathrm{Min} /+}$ control mice (Figure 12B, left) and strongly increased in adenomas of compound $\mathrm{APC}^{\mathrm{Min} /+}$; K19-BCL9-2 mice (Figure 12B, right). Moreover, transgenic expression of flag tagged BCL9-2 was found in intestinal tissue sections of adenomas of compound $\mathrm{APC}^{\mathrm{Min} /+} ; \mathrm{K} 19-\mathrm{BCL} 9-2$ mice (Figure $12 \mathrm{~A}+\mathrm{B}$ ). 
A.

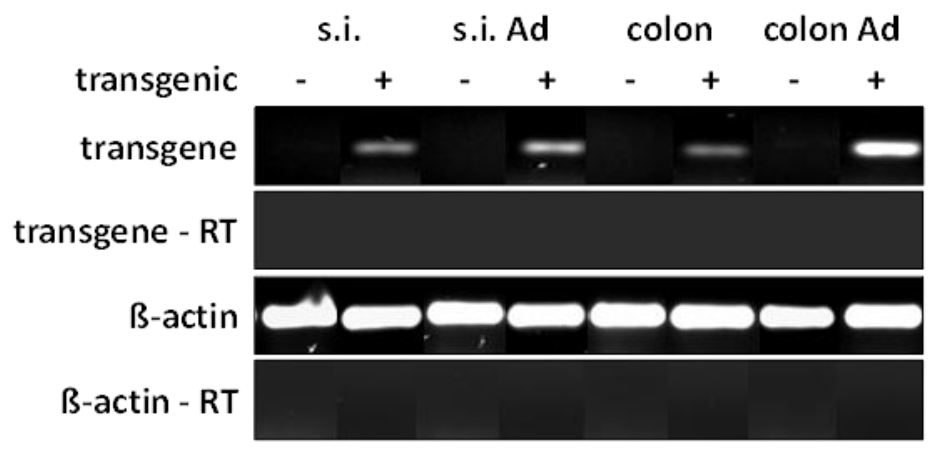

B.

$\mathrm{APC}^{\mathrm{Min} /+}$

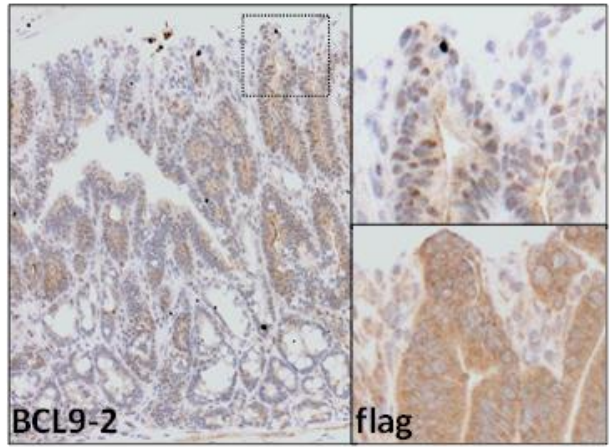

APCMin/+;K19-BCL9-2

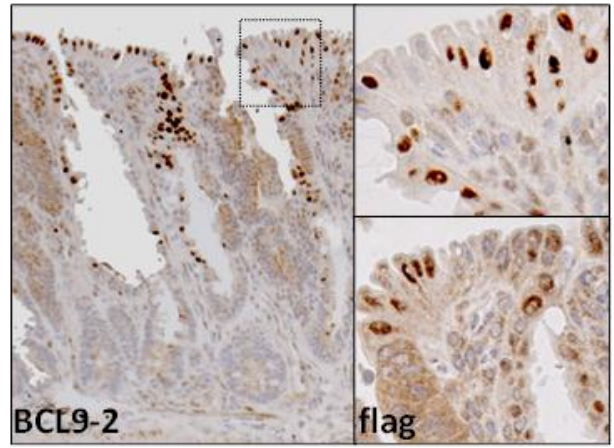

Figure 12: Transgenic BCL9-2 expression in compound $A P C^{\mathrm{Min} /+}$; K19-BCL9-2 intestinal adenomas and adjacent normal tissues compared to non-transgenic $\mathrm{APC}^{\mathrm{Min} /+}$ littermate controls

(A) Transgenic RNA expression in adenomas (Ad), normal tissues of the small intestine (s.i.) and colon was analyzed by RT-PCR. Specific primers were used to target the 3' flag tagged BCL9-2 transgene and Bactin as internal control. Templates without reverse transcription were used as controls (-RT). Transgenic and endogenous BCL9-2 protein expression was visualized by Immunohistochemistry on representative adenomas of the indicated genotypes using specific antibodies directed against BCL9-2 and the flag-tagged transgenic protein. (Brembeck, Wiese et al., 2011) 


\subsubsection{BCL9-2 overexpression promotes tumor formation and local invasion}

K19-BCL9-2 transgenic mice were crossed to C57BL/6-APC ${ }^{\mathrm{Min} /+}$ mice to investigate the impact of BCL9-2 on tumor development and progression. Overexpression of BCL9-2 resulted in a significant decrease of survival of compound $\mathrm{APC}^{\mathrm{Min} /+}$; K19-BCL9-2 mice compared to nontransgenic $\mathrm{APC}^{\mathrm{Min} /+}$ littermates (Figure 13). To follow the development of intestinal tumors due to aberrant BCL9-2 expression adenoma formation in the small intestine and colon of 5.5 month old compound $\mathrm{APC}^{\mathrm{Min} /+}$; K19-BCL9-2 mice and non-transgenic $\mathrm{APC}^{\mathrm{Min} /+}$ littermates were investigated in detail. H\&E staining and box plot analysis showed that transgenic mice exhibited significant increase both in number and in size of adenomas in the small intestine compared to age matched non-transgenic $\mathrm{APC}^{\mathrm{Min} /+}$ littermates (Figure $14 \mathrm{~A}$ and $\mathrm{B}$ ). A similar trend of tumor progression was observed in the colon, although $\mathrm{APC}^{\mathrm{Min} /+}$ mice do not frequently develop adenomas in the colon: $\mathrm{APC}^{\mathrm{Min} /+}$; K19-BCL9-2 formed a slight, but not significantly higher number of larger adenomas than $\mathrm{APC}^{\mathrm{Min} /+}$ mice (Figure 14B).

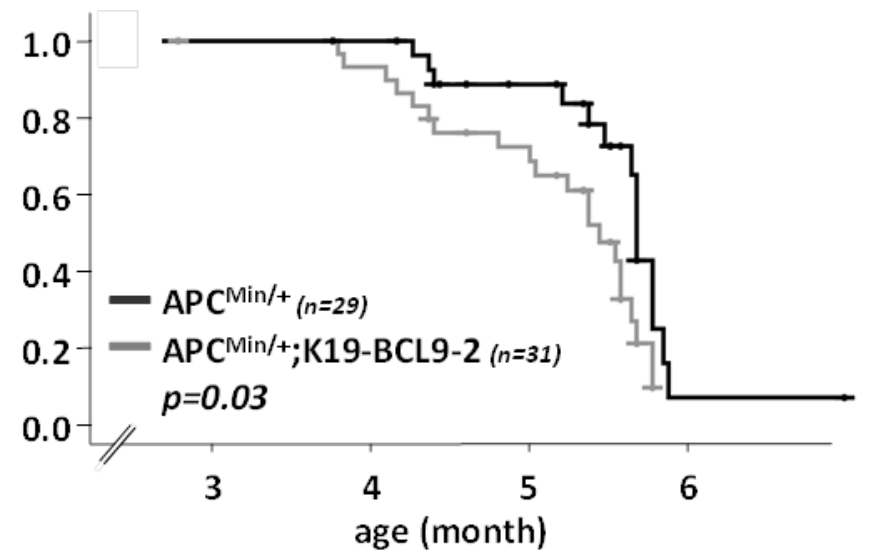

Figure 13: BCL9-2 overexpression leads to impaired survival of compound APC ${ }^{\mathrm{Min} /+}$; K19-BCL9-2 mice Kaplan-Meier survival analysis of compound mutant $(n=31)$ and of littermate APC ${ }^{\mathrm{Min} /+}$ control mice $(n=29)$. The survival was defined as the time point of death or the time point at which mice were sacrificed due to severe tumor-related disease. Statistics were evaluated by the P log-rank test. (Brembeck, Wiese et al., 2011) 
A. $\mathrm{APC}^{\mathrm{Min} /+}$
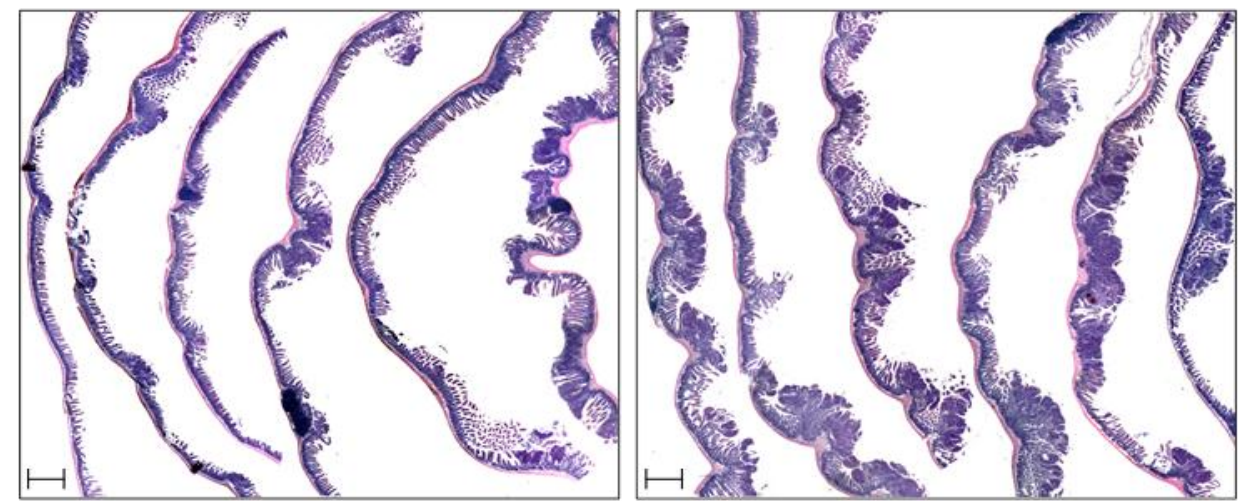

B.
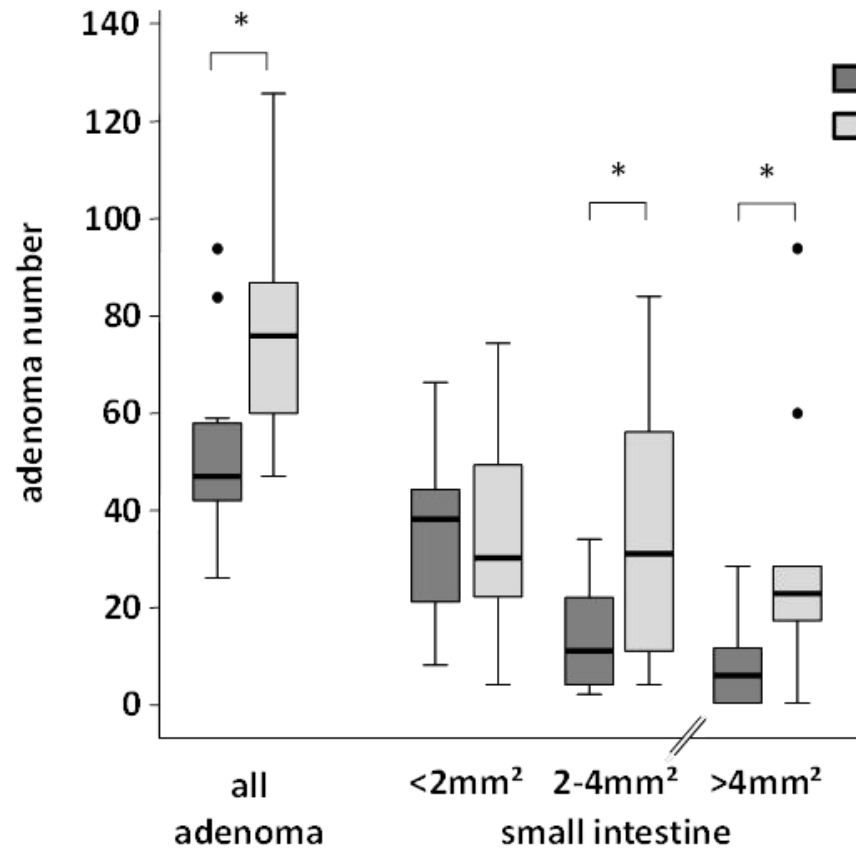

age $5.5 \pm 0.5$ month

$-20$

$\mathrm{APC}^{\mathrm{Min} /+}(n=13)$

$\mathrm{APC}^{\mathrm{Min} /+} ; \mathrm{K} 19-\mathrm{BCL}^{2}-2$ ( $\left.n=13\right)$

adenoma small intestine

colon

Figure 14: BCL9-2 overexpression promotes adenoma formation in number and size in compound APCMin/+; K19-BCL9-2 mice

(A) H\&E staining of representative small intestines of APCMin/+; K19-BCL9-2 and non-transgenic controls show an overview of the severe formation of adenomas in transgenics, (bar $-1 \mathrm{~mm}$ ). (B) Box blot analyses showing the total number of adenomas (left) and adenoma formation for the indicated sizes in the small intestine and colon (right) of 5,5 $\pm 0,5$ month old transgenic $(n=13)$ and non-transgenic littermates $(n=13)$. For calculation of statistical significances the Whitney-U test test was used $\left({ }^{*} p \leq 0.05\right)$. (Brembeck, Wiese et al., 2011)

$\mathrm{APC}^{\mathrm{Min} / \mathrm{H}}$ mice are known to develop benign tumors with well-formed boundaries (Moser et al., 1990). In contrast, adenomas from compound K19-BCL9-2 mutant mice already showed local invasion with an incidence of $14 \%$, which was never observed in control mice. CoImmunofluorescence of $\mathrm{APC}^{\mathrm{Min} /+}$; K19-BCL9-2 intestinal sections with pan-cytokeratin and smooth muscle actin monitored invasive tumors that disrupted the basal membrane. Invasive tumors grew beyond the mucosa into the submucosa (Figure 15A, middle) or even into the 
muscularis (Figure 15A, right). In contrast, adenomas of $\mathrm{Apc}^{\mathrm{Min} /+}$ mice were restricted to the mucosa with an intact basal membrane (Figure 15A, left). Immunohistochemical analyses on sections of invasive tumors of compound $\mathrm{APC}^{\mathrm{Min} /+}$; K19-BCL9-2 revealed high expression of BCL9-2 and of nuclear ß-catenin at the invasive front (Figure 15B).

A.

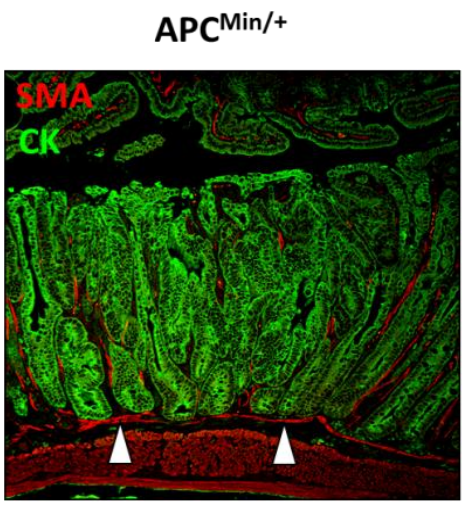

B.

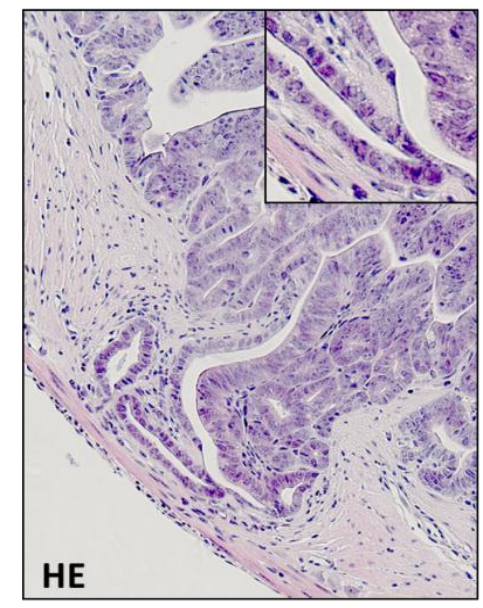

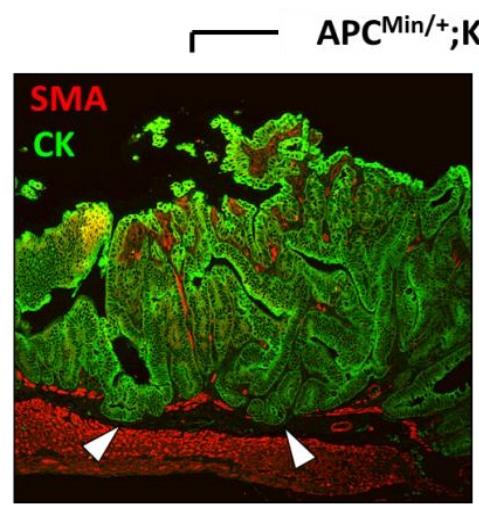

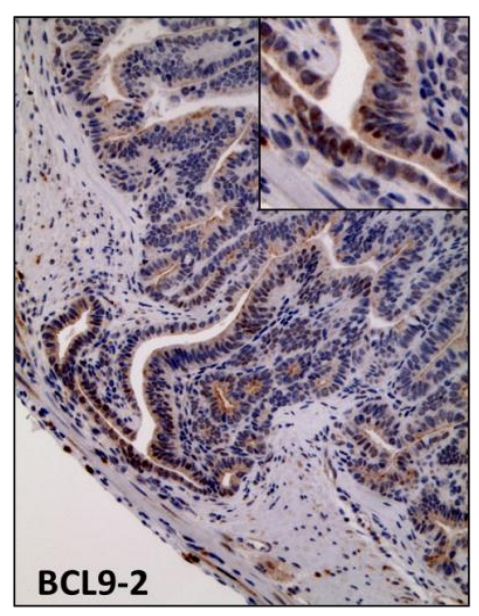

K19-BCL9-2 $\longrightarrow$
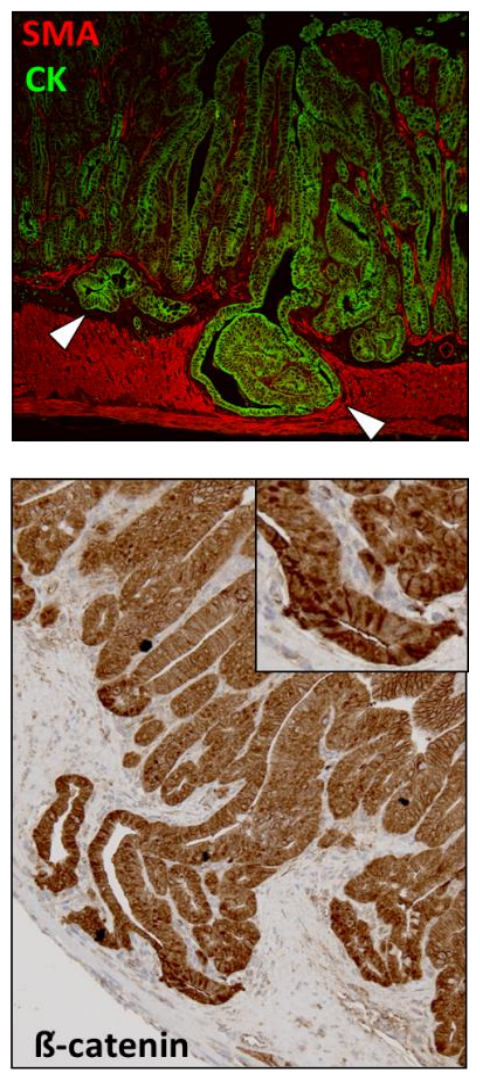

Figure 15: BCL9-2 overexpression induced invasion in compound $\mathrm{APC}^{\mathrm{Min} /+}$; K19-BCL9-2 mice

(A) Co-immunofluorescence staining of smooth muscle actin (SMA, red) and pan-cytokeratin (CK, green) on representative adenomas of an APCMin/+ control mice (left) and of compound APCMin/+; K19-BCL92 mice (middle, right). Arrowheads indicate the invasion into the basal membrane ( $200 \times$ magnification). (B) H\&E (left) staining and immunohistochemistry (middle and right) on an invasive adenoma of compound APCMin/+; K19-BCL9-2 mice of BCL9-2 and B-catenin, as indicated (400 x magnification). (Brembeck, Wiese et al., 2011)

These data demonstrate that overexpression of BCL9-2 in vivo promotes tumor progression in the intestine.

\subsection{BCL9 and BCL9-2 protein expression in colon cancer cell lines}

The majority of human colorectal carcinomas exhibited BCL9-2 overexpression (Figure 11) (Brembeck et al., 2011). To investigate the molecular function of BCL9 proteins for colorectal 
carcinogenesis, different colon carcinoma cell lines were analyzed for endogenous expression of BCL9-2 and the homologue BCL9. Therefore, our newly developed antibodies were analyze endogenous BCL9 and BCL9-2 protein expression. Using Western Blot analysis the proteins were detected at approximately $180 \mathrm{kDa}$. Highest protein expression of BCL9-2 was observed in cell lines with mutant APC (Rowan et al., 2000), e.g. in human SW480, CaCo2, HT29, DLD1 cells or the murine CT26 cell line. Absence or lower BCL9-2 protein expression was detected in cell lines with ß-catenin stabilizing mutation (Kim et al., 2003), e.g. HCT116, SW48 and the cell line LS174T with mutated E-cadherin (Efstathiou et al., 1999) (Figure 16, upper panel). However, BCL9 was ubiquitously expressed at approximately equal levels in all analyzed cell lines (Figure 16 , middle panel).

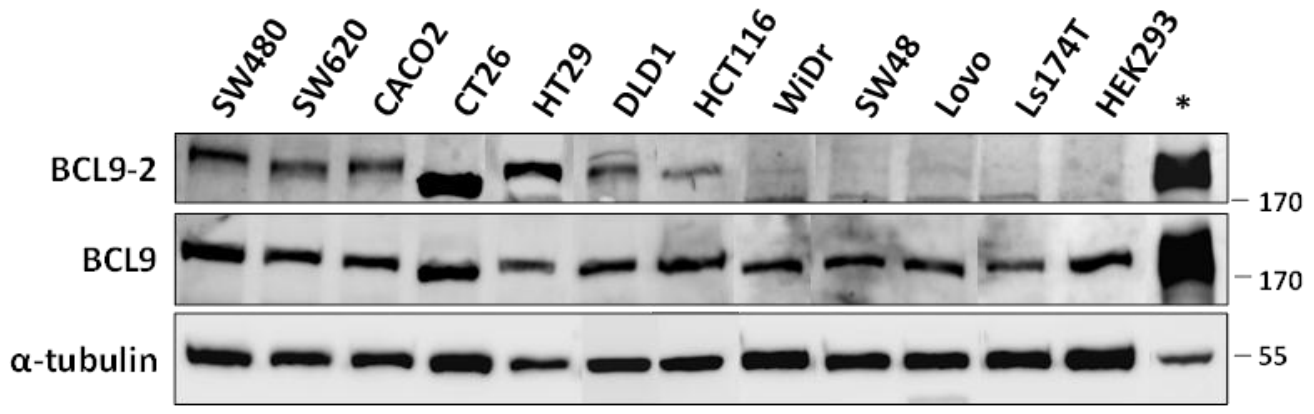

Figure 16: Expression of BCL9 proteins in different colon cancer cell lines

Western Blot analyses were performed with $60 \mu \mathrm{g}$ whole cell lysate obtained from the indicated cell lines, using specific antibodies for endogenous BCL9 and BCL9-2, respectively. As loading control $\alpha$ tubulin was used. Whole cell lysates obtained from transiently transfected HEK293 cells with BCL9 or BCL9-2 cDNA served as positive controls (indicated by an asterisk). (Brembeck, Wiese et al., 2011)

\subsection{Analysis of the dependency of the transcriptional control of $B$ - catenin, BCL9 and BCL9-2 on RNA Interference}

Colon cancer cell lines revealed high levels of ß-catenin caused by a mutation in APC and high levels of BCL9-2 (Figure 16). To investigate the transcriptional function of BCL9 proteins compared to the function of ß-catenin, RNA interference was used. A pool of 4 different nontargeting siRNAs was used as control. To cause minimal, unspecific effects $50 \mathrm{pmol}$ of modified Dharmacon ${ }^{\circledR}$ ON-TARGET plus ${ }^{\mathrm{TM}}$ siRNAs were used which were modified to reduce off-targeting caused by either sense or antisense strands. Further, off-target effects were minimized by the usage of a pool of two different siRNAs resulting in a minimal concentration of 25 pmol per siRNA. The potency of 4 single and pooled siRNAs to reduce the expression of BCL9, BCL9-2 and B-catenin transcripts in SW480 cells was investigated by qPCR. 

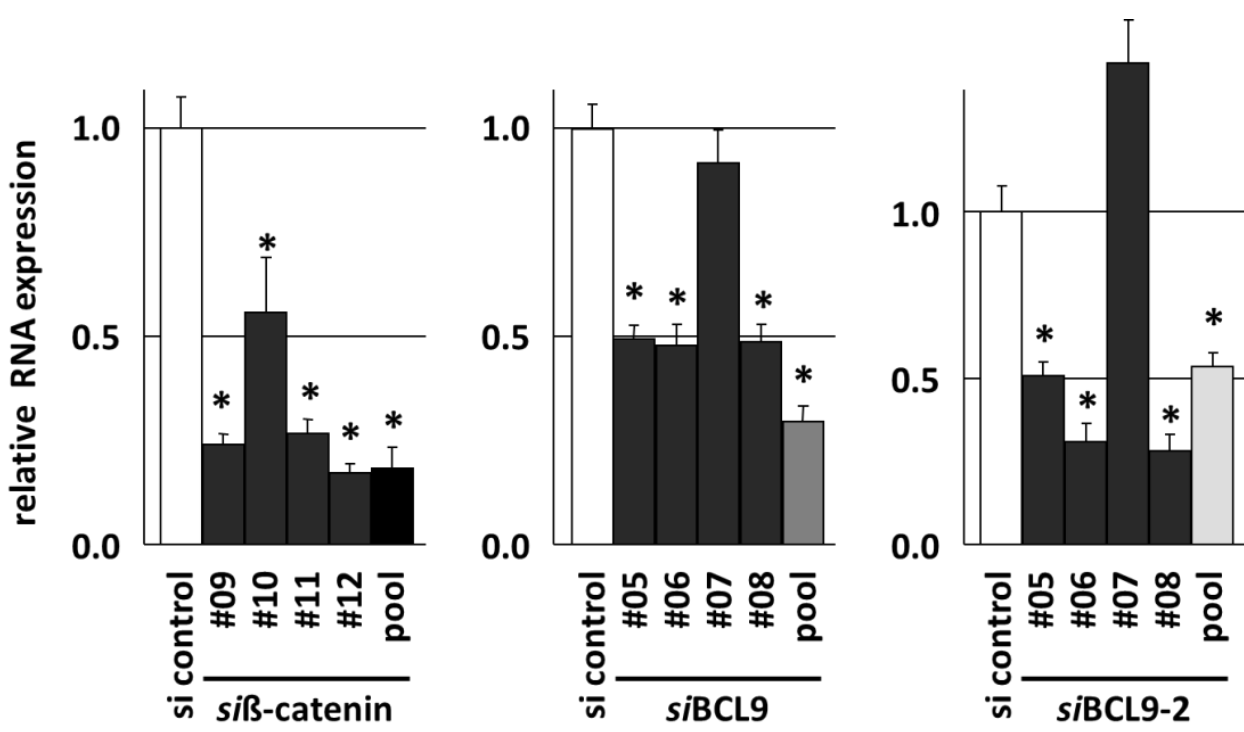

Figure 17: Knockdown efficiency of 4 single and pooled siRNAs targeting BCL9, BCL9-2 and B-catenin in SW480 cells

Quantitative real time PCR of SW480 showing the mean of least 3 independent experiments and their standard deviation after $48 \mathrm{~h}$ siRNA treatment. The relative expression of the indicated transcripts is shown as $\%$ of control siRNA-treated cells $\left({ }^{*} p \leq 0.05\right)$.

Pools containing four single siRNAs reduced the mRNA expression of $ß$-catenin to $20 \%$, of BCL9 to $30 \%$ and of BCL9-2 to $50 \%$ compared to control siRNA treated cells. RNA interference using te single siRNAs targeting ß-catenin, BCL9 and BCL9-2, revealed that only three of the four single siRNAs efficiently reduced the respective transcript (Figure 17). To increase the knockdown efficiencies, a pool of the two most efficient single siRNAs was used for further investigations (\#09 and \#12 for ß-catenin, \#06 and \#08 for BCL9 and \#06 and \#08 for BCL9-2 knockdown). The knockdown efficiency induced by these 2 pooled siRNAs targeting each transcript was further investigated on the mRNA and protein level (Figure 18). Treatment of SW480 cells with 2 specific siRNAs strongly reduced the RNA- and protein-levels of the respective targets (Figure 18A and B) to at least $25 \%$ compared to treatment of the cells with a pool of all 4 siRNAs. 
A.

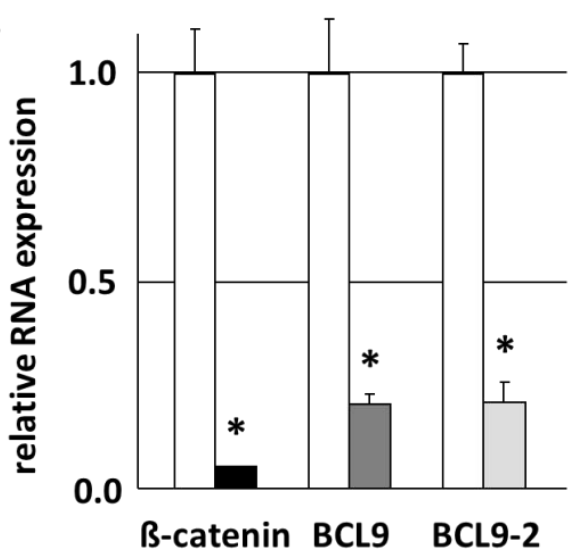

si control

si ß-catenin

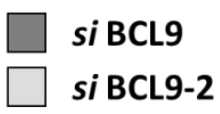

B.

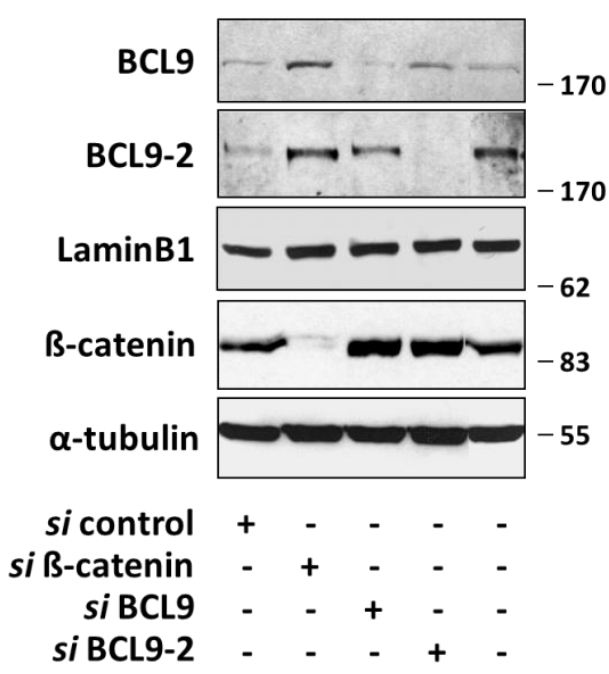

Figure 18: BCL9, BCL9-2 and ß-catenin knockdown in SW480 cells

(A) Quantitative real time PCR of SW480 showing the mean of least 3 independent experiments and their standard deviation after $48 \mathrm{~h}$ siRNA treatment. The relative expression of the indicated transcripts is shown as \% of control siRNA-treated cells $\left({ }^{*} p \leq 0.05\right)$. (B) Western Blot analysis of SW480 cells followed by $72 \mathrm{~h}$ RNA interference. Nuclear extracts were used for BCL9, BCL9-2, whole cell lysates for ßcatenin detection. LaminB1 and $\alpha$-tubulin served as internal controls. (Brembeck, Wiese et al., 2011)

Further investigations with these pools of specific siRNAs were carried out using different colon cancer cell lines such as SW480, DLD1, HCT116 and SW48. In addition, the breast cancer cell line MCF7 was used, which contains high levels of BCL9-2 protein (Zatula and Brembeck, unpublished).

\subsection{BCL9 and BCL9-2 knockdown revert the mesenchymal malignant phenotype of cancer cell lines}

The investigated cancer cell lines showed a characteristic change of the morphology after treatment with siRNA targeting BCL9 and BCL9-2 or B-catenin after $72 \mathrm{~h}$. In comparison to the more mesenchymal-like morphology of control siRNA treated cells (Figure 19, upper panel), cells in which BCL9-2 was knocked down showed a more epithelial-like phenotype characterized by a flattened appearance and tight cell-cell contacts. This phenotype was also observed for MCF7 breast cancer cells, which are highly positive for BCL9-2 (Zatula and Brembeck, unpublished). Treatment with BCL9 siRNA led to similar, but less pronounced morphological changes like BCL9-2 knockdown (Figure 19, middle panels). In contrast, knockdown of ß-catenin enhanced the mesenchymal-like, spindle-shaped morphology of the cells that did not form tight cell-contacts (Figure 19, bottom panel). 
These data indicate that BCL9-2 overexpression enhances the mesenchymal phenotype in colon and breast cancers possibly mediated by the promotion of epithelial-mesenchymal transition.

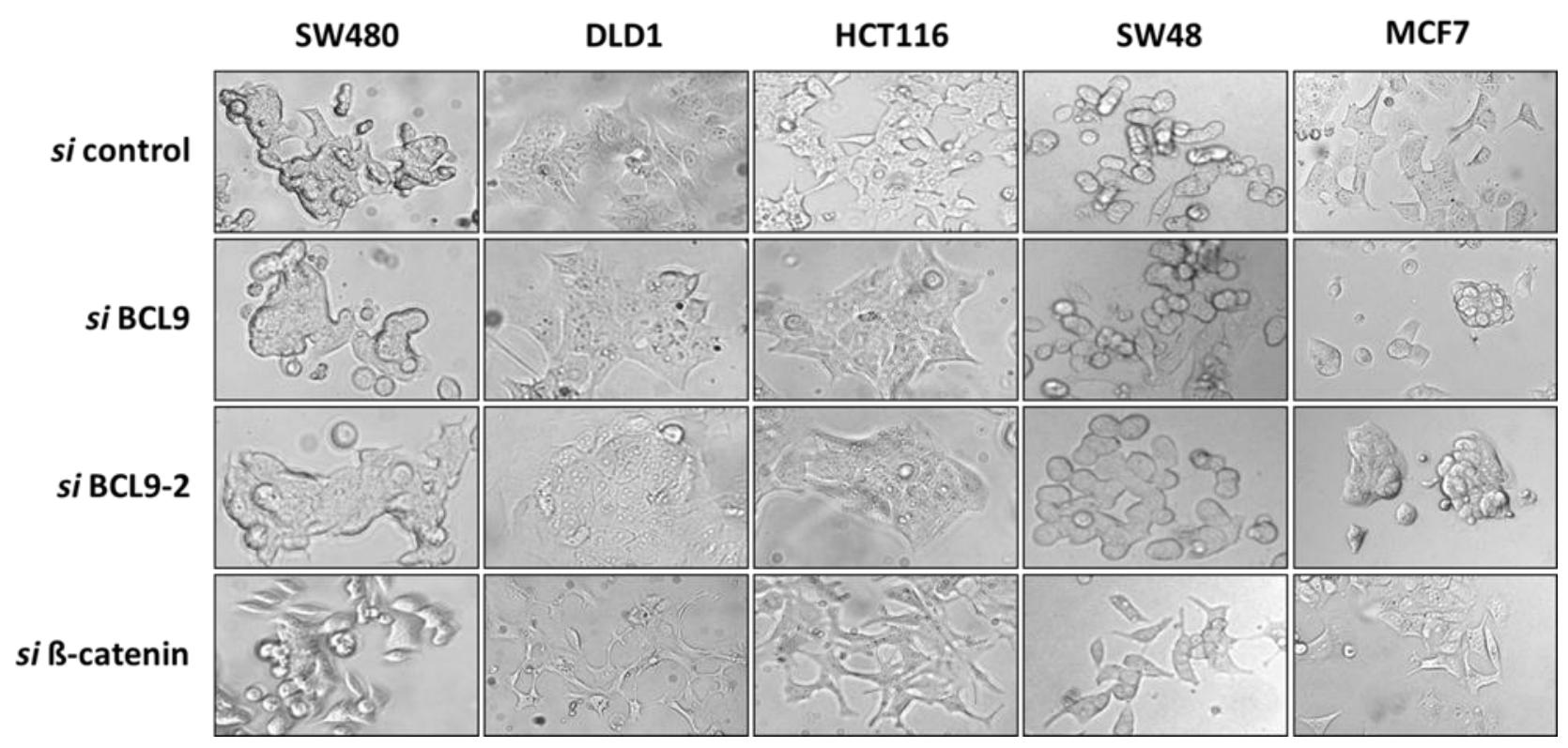

Figure 19: Knockdown of BCL9 and BCL9-2 induces characteristic morphological changes

Bright field images of human colon (SW480, DLD1, HCT116, and SW48) and breast (MCF7) cancer cells that were treated with a pool of 2 specific siRNAs against $\beta$-catenin, BCL9, BCL9-2 for 72 hours and nontargeting control siRNA (200x magnification).

\subsection{BCL9 and BCL9-2 are not target genes of ß-catenin}

To investigate, if BCL9 and BCL9-2 gene expression is regulated by Wnt/ß-catenin signaling, SW480 were treated with the respective siRNAs for $48 \mathrm{~h}$. The target gene transcription was determined by quantitative real-time PCR.

ß-catenin knockdown did not reduce BCL9 or BCL9-2 expression (Figure 20A) and neither, BCL9 nor BCL9-2 had an influence on each other's transcription. Interestingly, BCL9-2 expression was significantly induced after knockdown of ß-catenin (Figure 20A).

These results show that BCL9 and BCL9-2 are not target genes of $\beta$-catenin signaling in colon cancer cells. Moreover, ß-catenin appears to negatively regulate BCL9-2 expression. 


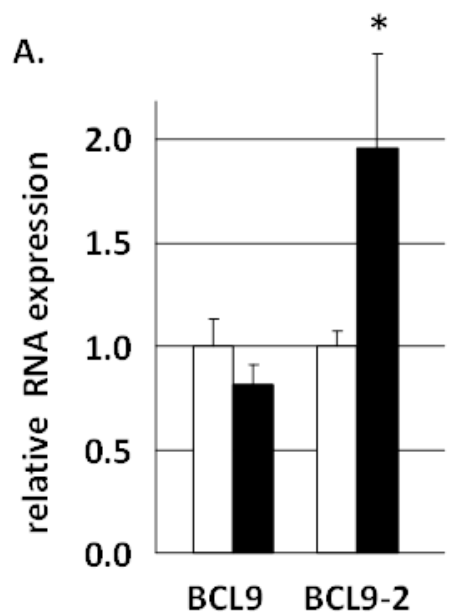

B.

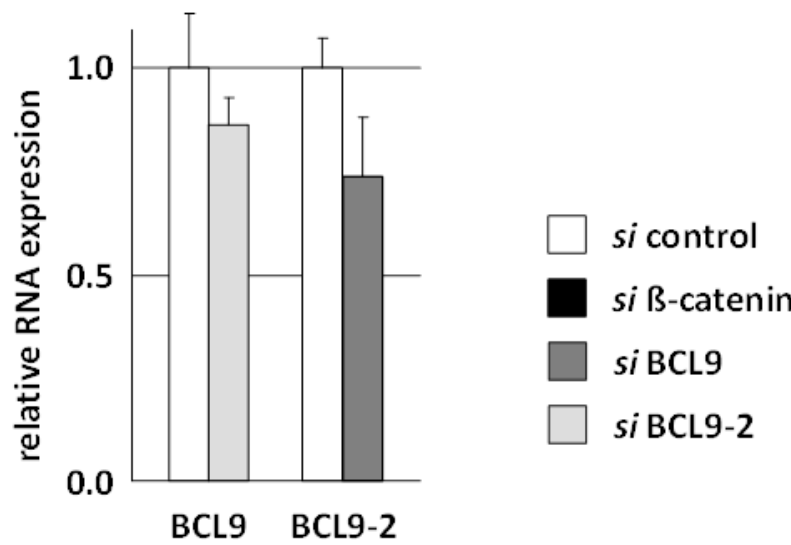

Figure 20: BCL9 and BCL9-2 are not target genes of B-catenin

Expression of BCL9 and BCL9-2 was assessed as \% of control siRNA-treated cells following knockdown of (A) B-catenin, (B) BCL9 and BCL9-2 for 48h. Quantitative real time PCRs show the mean of at least 3 independent experiments and their respective standard deviation $\left({ }^{*} \mathrm{p} \leq 0.05\right)$. (Brembeck, Wiese et al., 2011)

\section{7 ß-catenin/Wnt-signaling activity correlates with the expression level of BCL9-2 in human colon cancer cells}

BCL9-2 co-activates the Wnt/B-catenin signaling pathway in HEK293 cells (Brembeck et al., 2004). For that reason, Luciferase assays with different ß-catenin-dependent reporters were performed in colorectal cancer cell lines to examine the dependency of the ß-catenin/Wntsignaling activity on the BCL9 proteins in colon cancer cells.

Colorectal cancer cell lines with different levels of BCL9-2 were examined (Figure 16): SW480 cells with high, DLD1 cells with moderate, HCT116 cells with low and SW48 cells with no BCL92 expression. BCL9 was equally expressed in the cell lines (Figure 16). The cell lines were treated for $48 \mathrm{~h}$ with siRNAs targeting ß-catenin, BCL9 and BCL9-2, respectively, before they were transfected with 3 different Luciferase-reporters for further $36 \mathrm{~h}$. Since the promoters of the synthetic TOP/FOP reporter harbors three optimal, functional (TOP) or mutated (FOP) LEF/TCF binding sites (Molenaar et al., 1996) that serve as a direct readout for canonical Wntsignaling. The regulation of well-known Wnt/ß-catenin signaling target genes was determined using a LUC-plasmid containing $5600 \mathrm{bp}$ of the Axin2- (Jho et al., 2002) or -136pb of the CyclinD1- (Shtutman et al., 1999) promoter that consequently control the Luciferase-reporters gene expression. 
SW480 cells expressing highest levels of BCL9-2 showed also highest Wnt/B-catenin activity, as determined by the TOP/FOP Luciferase-reporters. The level of TOP/FOP and Axin2 reporter activity was significantly decreased after knockdown of BCL9 and BCL9-2. Interestingly, the CyclinD1 reporter was only dependent on ß-catenin, but not on the BCL9 proteins (Figure 21, left panel).

In DLD1 cells which contain moderate levels of BCL9-2 the TOP/FOP Luciferase-reporters was expressed with moderate levels and only knockdown of BCL9-2 and not BCL9 inhibited the Axin2 and TOP/FOP Luciferase-reporters.

In contrast, HCT116 with low and SW48 cells with absent BCL9-2 expression showed the lowest Wnt-activity. Accordingly, knockdown of neither, BCL9 or BCL9-2 had any influences on Axin2 and TOP/FOP reporter gene expression.

In conclusion, BCL9-2 expression levels correlate with ß-catenin/Wnt signaling activity in colon cancer cell lines. In addition, these data indicate that promoters with core Wnt-responsive elements such as the synthetic TOP/FOP and the Axin2 promoter are dependent on BCL9-2, but not the CyclinD1 promoter. 


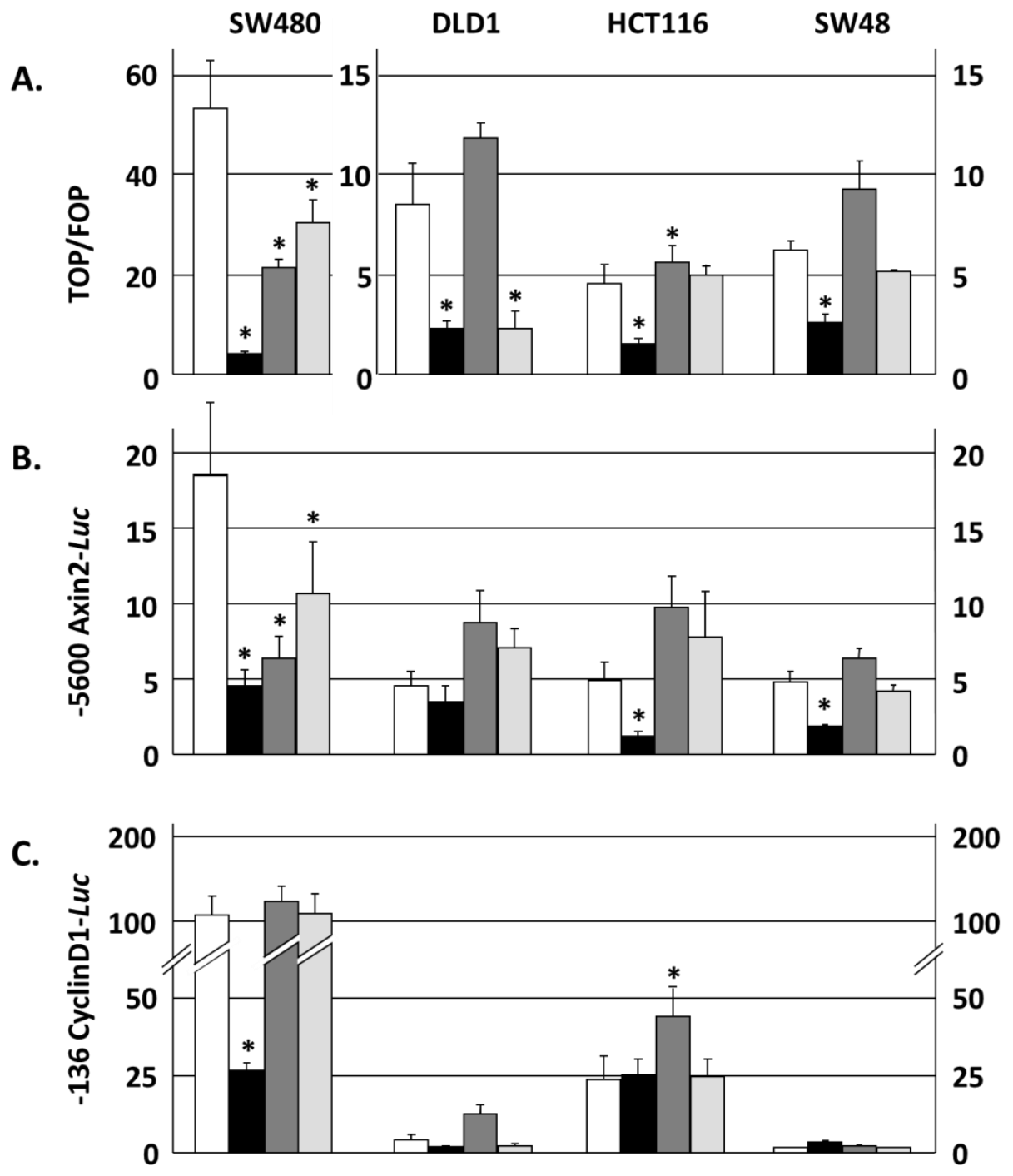

\begin{tabular}{llllll|} 
D. & APC & MT & MT & WT & WT \\
\cline { 3 - 5 } B-catenin & WT & WT & MT & MT \\
\cline { 5 - 6 }
\end{tabular}

BCL9-2 +\begin{tabular}{llll}
+++ & ++ & + & - \\
\cline { 3 - 4 }
\end{tabular}

$\begin{array}{llll}\text { BCL9 } \quad+++ & +++ & +++\end{array}$

\section{si control \\ si ß-catenin \\ si BCL9 \\ si BCL9-2}

Figure 21: BCL9-2 expression levels correlates with canonical Wnt signaling activity and reporter gene expression in different colon cancer cell lines

$(\mathrm{A}-\mathrm{C})$ For Luciferase assays the indicated cell lines were pretreated with siRNA targeting ß-catenin, BCL9 or BCL9-2 for 48h, followed by 36h transfection with LUC-reporters: (A) TOP/FOP, (B) -5600 bp Axin2-LuC and (C) 136 bp CyclinD1-Luc. (D) The cell lines harbor different mutations and BCL9 protein levels, as indicated (MT-mutated, WT-wild-type). Graphs show the fold change of empty control plasmid of the mean of at least four independent experiments with the respective standard deviation $\left(^{*} p \leq 0.05\right)$.(Brembeck, Wiese et al., 2011) 


\subsection{BCL9-2 regulates ß-catenin-dependent and -independent target genes}

To further understand the role of BCL9-2 and its homolog BCL9 in Wnt/ß-catenin signaling during tumorigenesis it is crucial to characterize the dependency of known Wnt/ß-catenin target genes on the BCL9 proteins. (Canonical Wnt-target genes are listed on the Wnt Homepage: http://www.stanford.edu)).

\subsubsection{BCL9-2 is not required for the expression of all canonical Wnt target genes and regulates ß-catenin independent genes in colon cancer cells}

To study the impact of the BCL9 proteins in colon cancer in detail, endogenous BCL9, BCL9-2 and ß-catenin expression was silenced in SW480 colon cancer cells for $48 \mathrm{~h}$ by RNA interference. The dependency of known Wnt/B-catenin target genes and the ephrin B ligands on ß-catenin and the co-factors was determined by $q P C R$. The ephrin B receptors (EPHB2/B3 and B4) are known to be expressed in intestinal crypts as Wnt-target genes in normal intestinal homeostasis. The ephrin B ligands (ephrinB1/B2 and B3) are no target genes of $ß$-catenin and expressed in normal intestinal villi where BCL9-2 protein is expressed. Therefore we tested whether the ephrin B ligands and receptors are target genes of $ß$-catenin and BCL9-2.

Different subsets of target genes of BCL9-2 and ß-catenin were identified (Figure 22):

The first set of genes was strongly down-regulated after knockdown of ß-catenin and BCL9-2 (Figure 19A), e.g. Axin2, EPHB2, CD44, TCF1, c-myc, BMP4 and Bambi. BCL9 knockdown also reduced the mRNA expression of this gene set with the exception of BMP4, while Bambi was reduced only slightly, but still significant.

The second set of Wnt/B-catenin target genes was not regulated by BCL9 or BCL9-2 (Figure 19B). B-catenin knockdown led to strongly reduced mRNA levels of LEF1, MSX2 and CyclinD1. Knockdown of BCL9-2 and BCL9 had no effect on the expression of the ß-catenin target genes LEF1, MSX2 and CyclinD1. This further supports our previous using a minimal Luciferasereporter - CyclinD1. Interestingly, LGR5 mRNA was slightly, but significantly reduced after BCL9, but not BCL9-2 knockdown.

The third set of genes consists of genes of the ephrin/EPHB family which was differentially regulated by ß-catenin and BCL9-2 (Figure 19C). Knockdown of BCL9-2 resulted in reduced 
expression of the analyzed genes, while ß-catenin knockdown had no influence or even increased the mRNA expression of the ephrin receptors EPHB3/B4 and the ephrin ligands B1, B2 and B3. In contrast, RNA Interference against BCL9 only led to a reduced mRNA expression of EPHB2, B3 and B4.

\section{A. ß-catenin/BCL9-2 target genes}

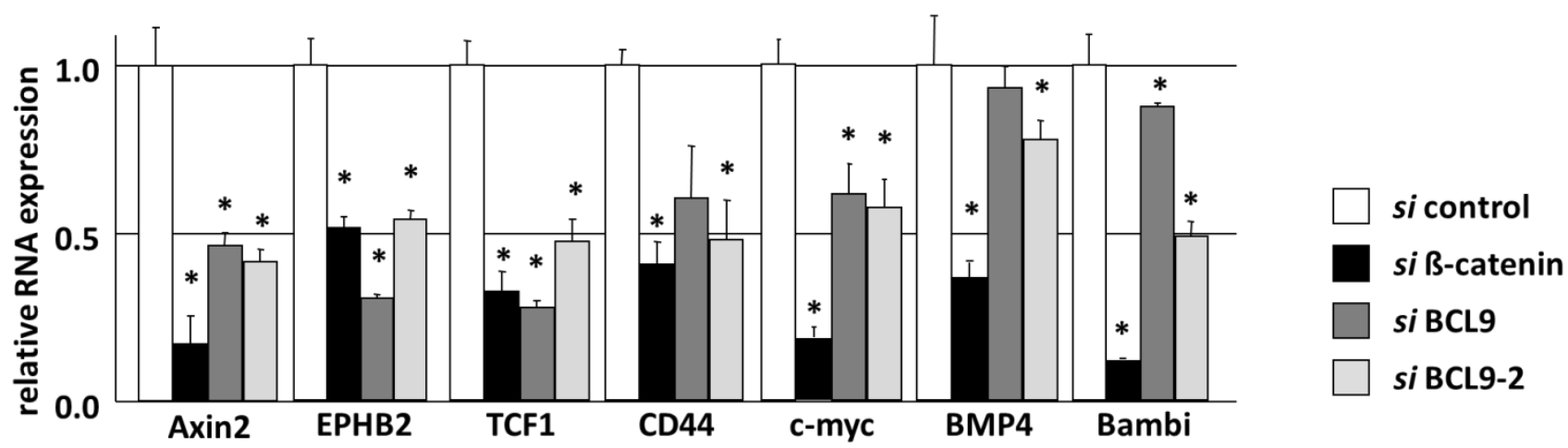

\section{B. ß-catenin target genes}

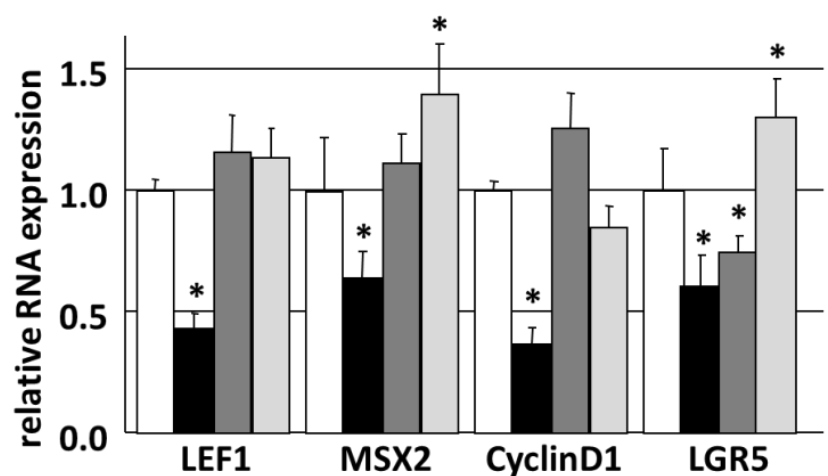

C. BCL9-2 target genes

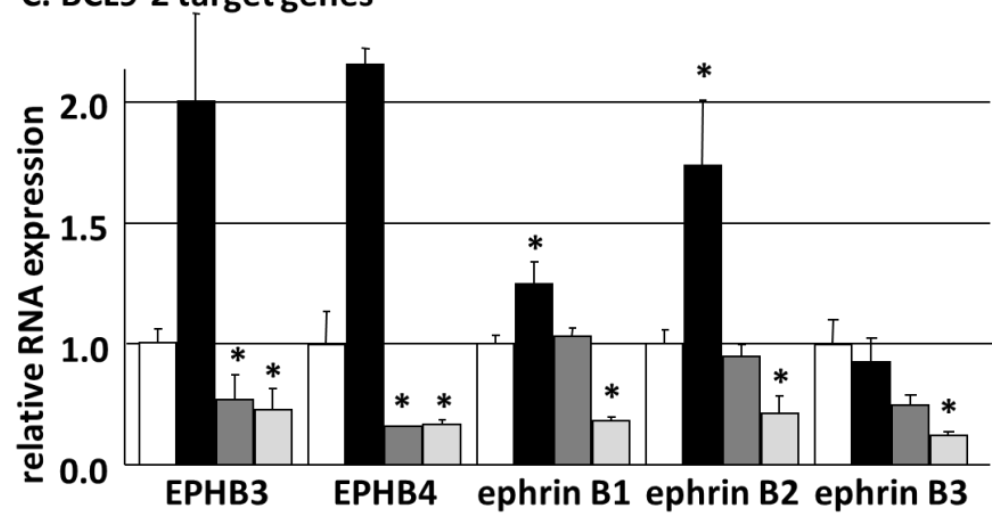

Figure 22: BCL9-2 regulated its own subset of target genes, apparently independent of ß-catenin Expression of the indicated genes was determined following knockdown of ß-catenin, BCL9 and BCL9-2 for $48 \mathrm{~h}$ relative to control siRNA-treated cells. Quantitative real time PCRs show the mean of least 3 independent experiments and their respective standard deviation ( ${ }^{*} p<0.05$ ). (A) Target genes of BCL9-2 and $ß$-catenin. (B) BCL9-2 independent $ß$-catenin target genes. (C) BCL9-2 and ß-catenin differentially regulated genes $\left({ }^{*} p \leq 0.05\right)$. (Brembeck, Wiese et al., 2011)

In summary, BCL9 and BCL9-2 regulate only a subset of Wnt/B-catenin target genes. Moreover, these data demonstrate that BCL9-2 also regulates ß-catenin independent target genes.

\subsubsection{B-catenin-dependent and -independent BCL9-2 target genes are expressed at} the invasive front of adenomas of compound K19-BCL9-2; APC ${ }^{\mathrm{Min} /+}$ mice

BCL9-2 contributes to adenoma formation from compound K19-BCL9-2; APC ${ }^{\mathrm{Min} /+}$ mice and induces locally invasive tumors which invade beyond the basal membrane into the submucosa 
or even into the muscularis (Figure 14 and Figure 15). Therefore, accumulation of ß-catenin in $\mathrm{APC}^{\mathrm{Min} /+}$ mice alone appears not to be sufficient to induce local invasions. However, BCL9-2 activates its own gene cluster independently from ß-catenin (Figure 19). Thereby some of these genes might be responsible for the development of invasive tumors in compound K19$B C L 9-2 ; A C^{\mathrm{Min} /+}$ mutant mice. To investigate whether BCL9-2 targets are expressed in the invasive areas of $\mathrm{BCL9}-2 ; \mathrm{APC}^{\mathrm{Min} /+}$ derived adenomas immunohistochemistry was performed using specific antibodies which detect the ß-catenin/BCL9-2 target genes Bambi and EPHB2 and the BCL9-2 target genes EPHB3 and B4. The invasive areas were previously stained positive for nuclear ß-catenin and BCL9-2 (Figure 15B) indicating high transcriptional activity.

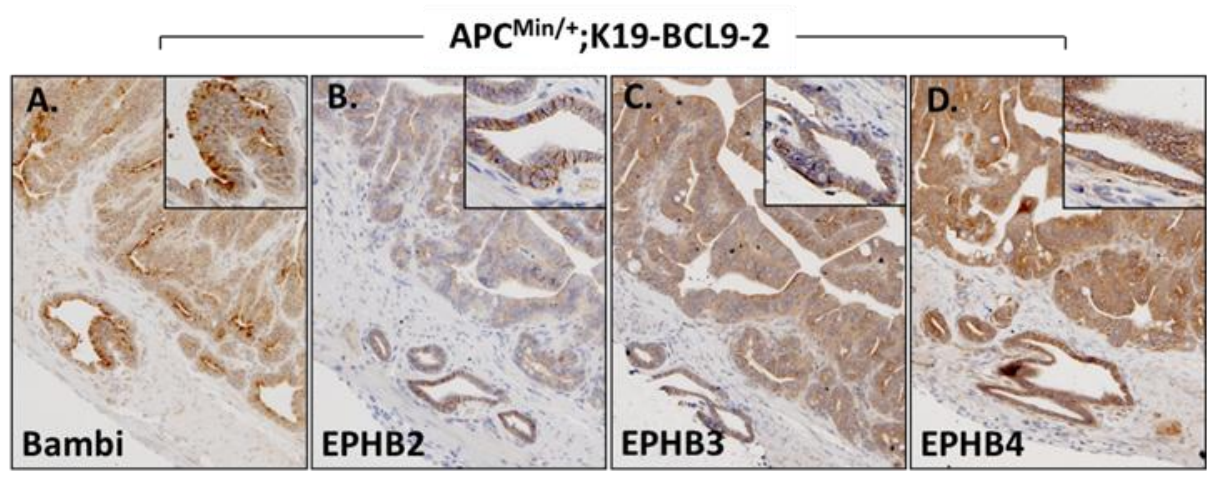

Figure 23: Expression of BCL9-2 targets in invasive areas of compound K19-BCL9-2; $A P C^{\mathrm{Min} /+}$ mice (A-D) Immunohistochemistry of (A) Bambi, the ephrin receptors (B) EPHB2, (C) EPHB3 and (D) EPHB4 on an invasive adenoma of compound $A P C^{\mathrm{Min} /+} ; \mathrm{K} 19-\mathrm{BCL9}-2$ mice (400x magnification).

Strong protein expression of the ß-catenin/BCL9-2 target genes Bambi and EPHB2 was observed in the invasive area compared to the central tumor area (Figure 23A and B). In addition, the ß-catenin independent BCL9-2 targets EPHB3 and EPHB4 (Figure $23 \mathrm{C}$ and D) were expressed in the invasive areas and in the central tumor.

In summary, BCL9-2 contributes to tumor formation and invasion probably by the regulation of an own set of target genes, which is partially independent of ß-catenin.

\subsection{Whole genome microarray analyses demonstrate that BCL9, BCL9-2 and ß-catenin activate different gene expression profiles}

BCL9-2 is up-regulated in the majority of colon cancers (Figure 11 and Figure 16) and contributes to cancer progression by promoting tumor growth (Figure 14) and invasion (Figure 15 and Figure 19). In addition, BCL9 and BCL9-2 regulate the expression of target genes 
independently from B-catenin (Figure 22). To understand the impact of BCL9-2 and its homolog BCL9 in comparison to ß-catenin during these processes, it is important to analyze the gene cluster which is affected by these proteins in comparison to known B-catenin target genes. (A list of described canonical Wnt-target genes is available from the Wnt Homepage http://www.stanford.edu)

To explore the gene signature of BCL9 proteins and B-catenin in tumorigenesis human whole genome microarrays were analyzed. Colon cancer cell lines with different levels of BCL9-2 were examined. Expression data from previous unpublished human whole genome microarrays of SW480 cells, which exhibit the highest BCL9-2 protein level, were already available (Brembeck, unpublished). These data were completed for DLD1 colon cancer cells with moderate and HCT116 cells with low BCL9-2 protein levels. In addition, our current studies MCF7 breast cancer cells to express also very high levels of BCL9-2 protein (Zatula and Brembeck, unpublished). Since, MCF7 cells did not show measureable active canonical Wnt signaling, as determined by TOP/FOP reporter gene assays, these breast cancer cells served as an additional model to investigate ß-catenin independent functions of BCL9-2.

The gene expression profile of the described cell lines was determined in triplicates after knockdown of endogenous BCL9, BCL9-2 and ß-catenin for 48h using a pool of two different siRNAs, respectively, and normalized to cells treated with a pool of four non-targeting control siRNAs. The efficacy of all siRNAs was previously tested in all used cell lines by qPCR (data not shown). The gene signatures in the identified whole genome micro arrays resulting from knock down of B-catenin, BCL9 and BCL9-2 in the different cell lines, were subsequently analyzed. Each data set consisted of biological triplicates. The data sets for each treatment (siß-catenin, siBCL9 and siBCL9-2) were relativized to the data set originated from sicontrol treated cells. For further analyses only genes were used, which were expressed with fold discovery rate (FDR $\hat{=}$ adjusted $p$-value) $\leq 0.05$. In addition, only genes which were expressed with a minimum of a moderate expression level of 7 from total 15 were further analyzed.

\subsection{1 ß-catenin and BCL9/BCL9-2 induce different gene sets in cancer cells}

To investigate, whether BCL9 and BCL9-2 regulate specific sets of target genes independently of ß-catenin, Venn diagram analyses were performed. These illustrated the overlap of all genes that were significantly reduced $\geq 1.5$ fold following knockdown of ß-catenin, BCL9 and BCL9-2 in SW480, DLD1 and MCF7 cells (Figure 24). In addition, heat map analyses compared all genes by a color coded expression score. To this end all genes were included, that showed $\geq 2$ fold 
down-regulation following knockdown of ß-catenin and of BCL9-2 in SW480 and MCF7 cells (Figure 25).

The microarray analyses confirmed the distinct transcriptional roles of $ß$-catenin and of BCL9/BCL9-2. The induced gene sets of BCL9 and BCL9-2 were only in part overlapping with the gene cluster of ß-catenin (Figure 24 and Figure 25). Only $4 \%$ of BCL9-2 and 6\% of BCL9 dependent genes were present in the gene set regulated by ß-catenin in SW480. In DLD1 cells $13 \%$ of BCL9-2 and $14 \%$ of BCL9 target genes were regulated by ß-catenin. In addition, BCL9 and BCL9-2 itself shared almost 10\% of their target genes in SW480 and DLD1 (Figure 24, left and middle).

SW480

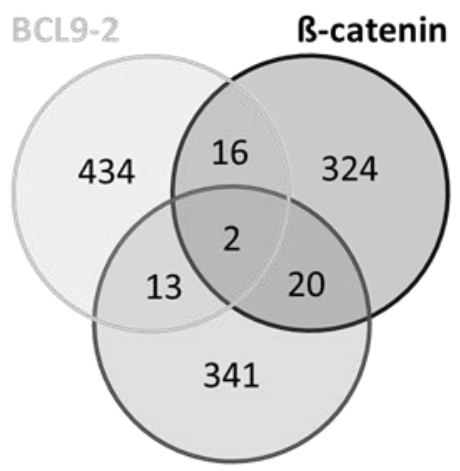

BCL9
DLD1

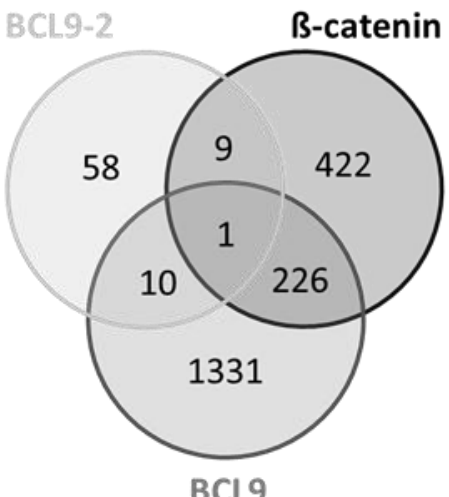

MCF7

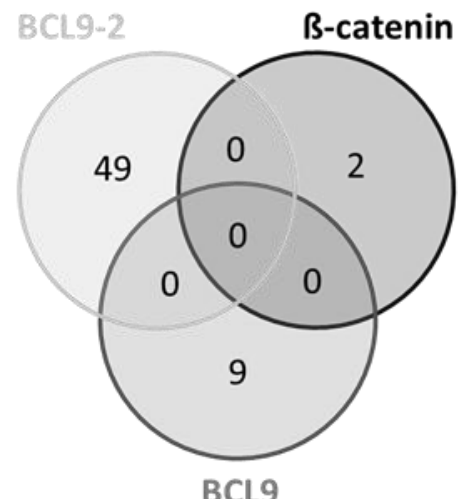

Figure 24: ß-catenin and BCL9/BCL9-2 induce different gene sets in cancer cell lines

Venn diagrams illustrating the overlap of $\geq 1.5$ fold down-regulated genes in SW480, DLD1 and MCF7 cells following knockdown of the proteins for $48 \mathrm{~h}$, as indicated (FDR $\leq 0.05$ ).

Moreover, knockdown of ß-catenin in MCF7 led to reduction only one gene covered by two oligonucleotides and which represented B-catenin itself. In addition, BCL9 knockdown in MCF7 appeared to be less effective in transcriptional modulation compared to BCL9-2, since only nine genes were regulated by BCL9 compared to 49 genes which appeared in the BCL9-2 gene set (Figure 24 and Figure 25, right). In contrast, knockdown of BCL9 in DLD1 cells resulted in more than 1500 differentially regulated genes, while BCL9-2 knockdown led to reduction of only 78 genes. Since DLD1 cells contain moderate levels of BCL9-2, BCL9 appeared to have a stronger impact on gene transcription than BCL9-2 (Figure 24, middle).

The heat map analyses for all highly significant regulated genes revealed that BCL9-2 indeed shares many genes with the cluster of ß-catenin in SW480 colon cancer cells. However, BCL9-2 significantly regulates several additional target genes independently of ß-catenin (Figure 25, left). 
Moreover, heat map analyses showed that there is almost no detectable transcriptional function of ß-catenin in MCF7 cells. In contrast, BCL9-2 appeared to be a potent transcription factor for the regulation of target genes in breast cancer cells (Figure 25, right).

These results demonstrate that $\mathrm{BCL} 9-2$ dependent transcriptional regulation in different cancer cells does not rely completely on ß-catenin and that BCL9 and BCL9-2 are able to transactivate a gene set independently of ß-catenin/Wnt signaling.

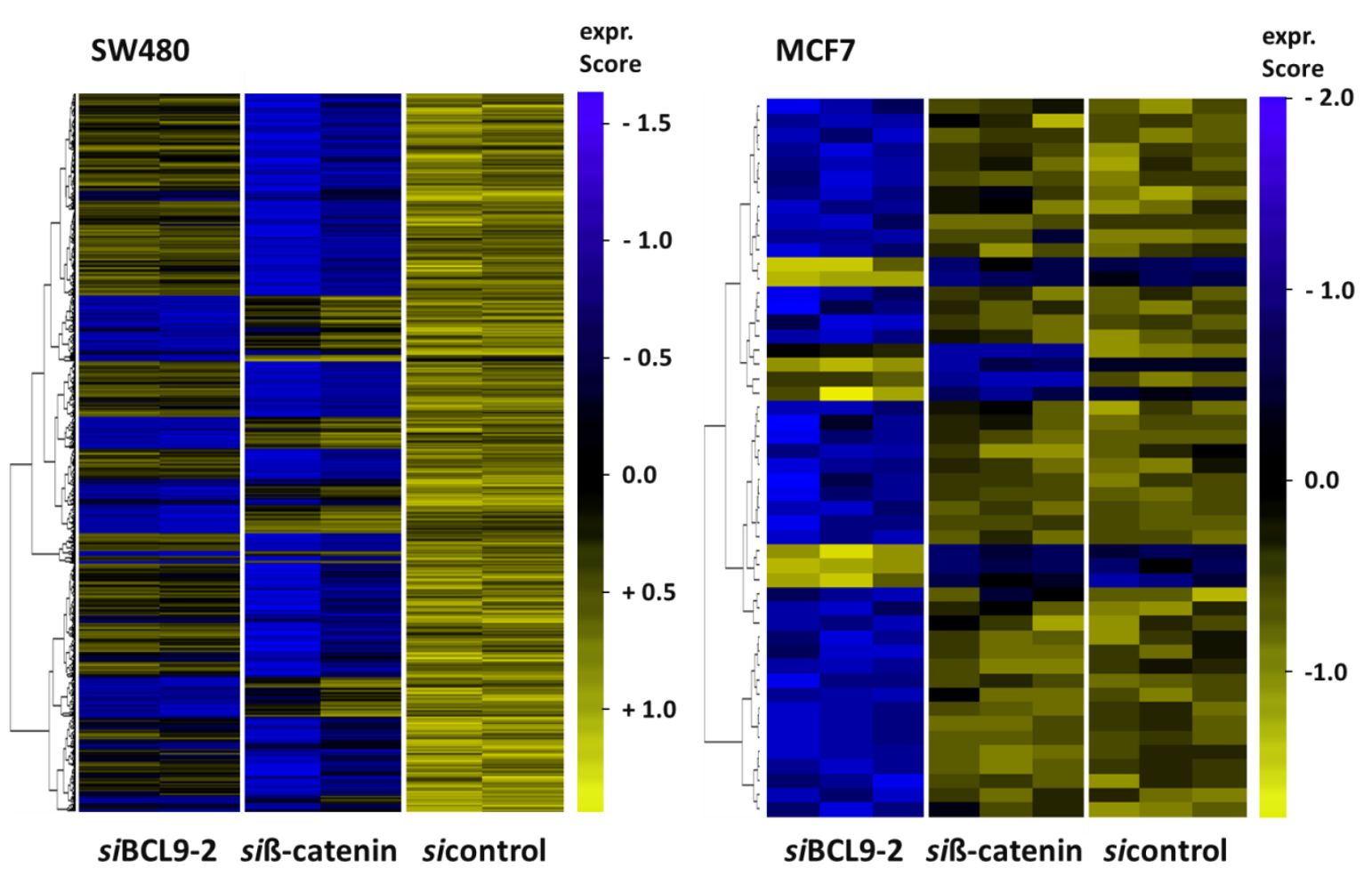

Figure 25: BCL9-2 regulates target genes independently of ß-catenin.

Heat maps show all $\geq 2$ fold down-regulated genes in SW480 and MCF7 cells after knock down of ßcatenin and BCL9-2 (FDR $\leq 0.05$ ). The expression of genes is shown in comparison to non-targeting siRNA treated samples and depicted by a color coded expression score.

In summary, the induced gene signature resulting from knockdown of BCL9 and BCL9-2 in different cancer cells are only in part overlapping with the gene cluster of regulated by ßcatenin. In addition, the number of BCL9-2 regulated genes correlated positively with the expression level of BCL9-2 in the different cancer cell lines. 


\subsection{Novel BCL9-2 target genes in cancer cells identified by microarray analyses}

\subsubsection{Comparison of the gene expression profile of cancer cell lines and of tumors from the intestine specific double knock out of VilCre; $\mathrm{BCL} 9^{-/-} / \mathrm{BCL9-2^{-/- }}$ mice}

Deka and colleagues developed a intestine specific BCL9 $9^{-1-} / \mathrm{BCL9}-2^{-1-}$ knockout mouse model in which they chemically induced dysplastic colon adenomas (Deka et al., 2010). Tumors deriving from wild-type and $\mathrm{BCL}^{-/} / \mathrm{BCL} 9-2^{-/-}$mice were analyzed by comparative gene expression profiling and the regulated genes were publicly available for our further analyses. This model reflects the in vivo situation which is mimicked by siBCL9 and siBCL9-2 treatment of our colon cancer cell lines. We compared the gene expression profile of $\mathrm{BCL} 9^{-1} / \mathrm{BCL} 9-2^{-1-}$ derived tumors and siBCL9, siBCL9-2 and siß-catenin treated SW480 cells to investigate the regulatory functions of BCL9 and BCL9-2 in comparison to ß-catenin in colon cancer.

We compared target genes of BCL9 and BCL9-2, which were significantly $\geq 1.5$ fold downregulated in SW480 cells and target genes, which displayed $\geq 2$ fold decreased transcription, originating from the expression profile of $\mathrm{BCL}^{-/} / \mathrm{BCL} 9-2^{---}$mice derived tumors. The graphs show the fold change of the respective genes compared to SW480 cells treated with nontargeting siRNA treated or tumors derived from wild-type mice, respectively (Figure 26).

From 1200 genes, which appeared in the expression profile of $\mathrm{BCL9} 9^{-/-} / \mathrm{BCL9}-2^{-1-}$ mice derived tumors, 28 were also present in the gene sets of siBCL9/SiBCL9-2-treated SW480 cells. Out of these 28 genes, only knockdown of BCL9 resulted in reduction of 5 specific genes, like the gene of ras-related protein 2b (RAP2B) (Figure 26C), whereas only knockdown of BCL9-2 led to the reduction of 19 genes such as the gene of transforming growth factor beta 3 (TGFB3) (Figure 26D). Interestingly, only 4 genes appeared in the gene set of siß-catenin-treated SW480 cells, such as Axin2 (Figure 26A and B). 
A.

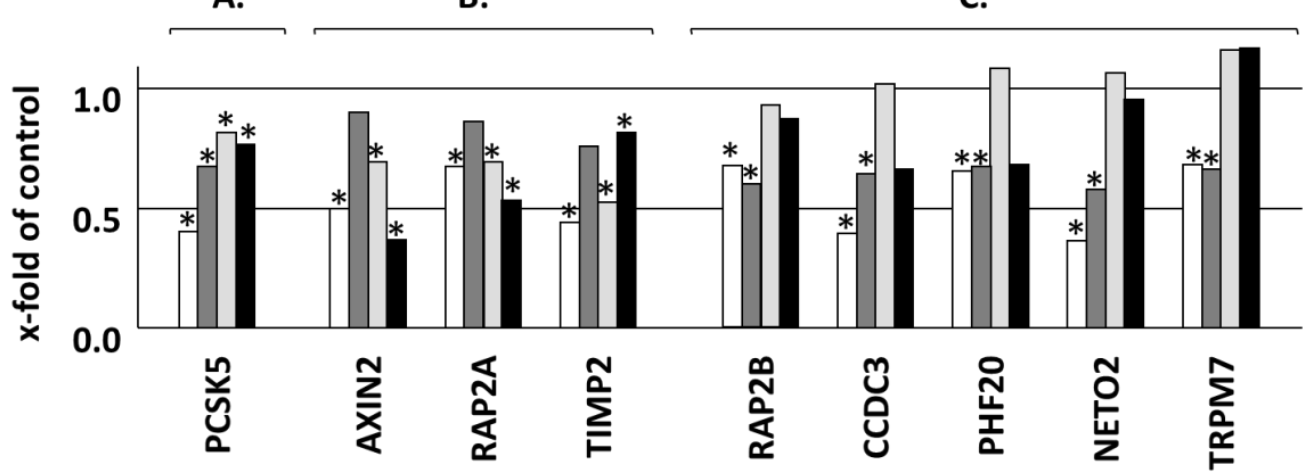

D.

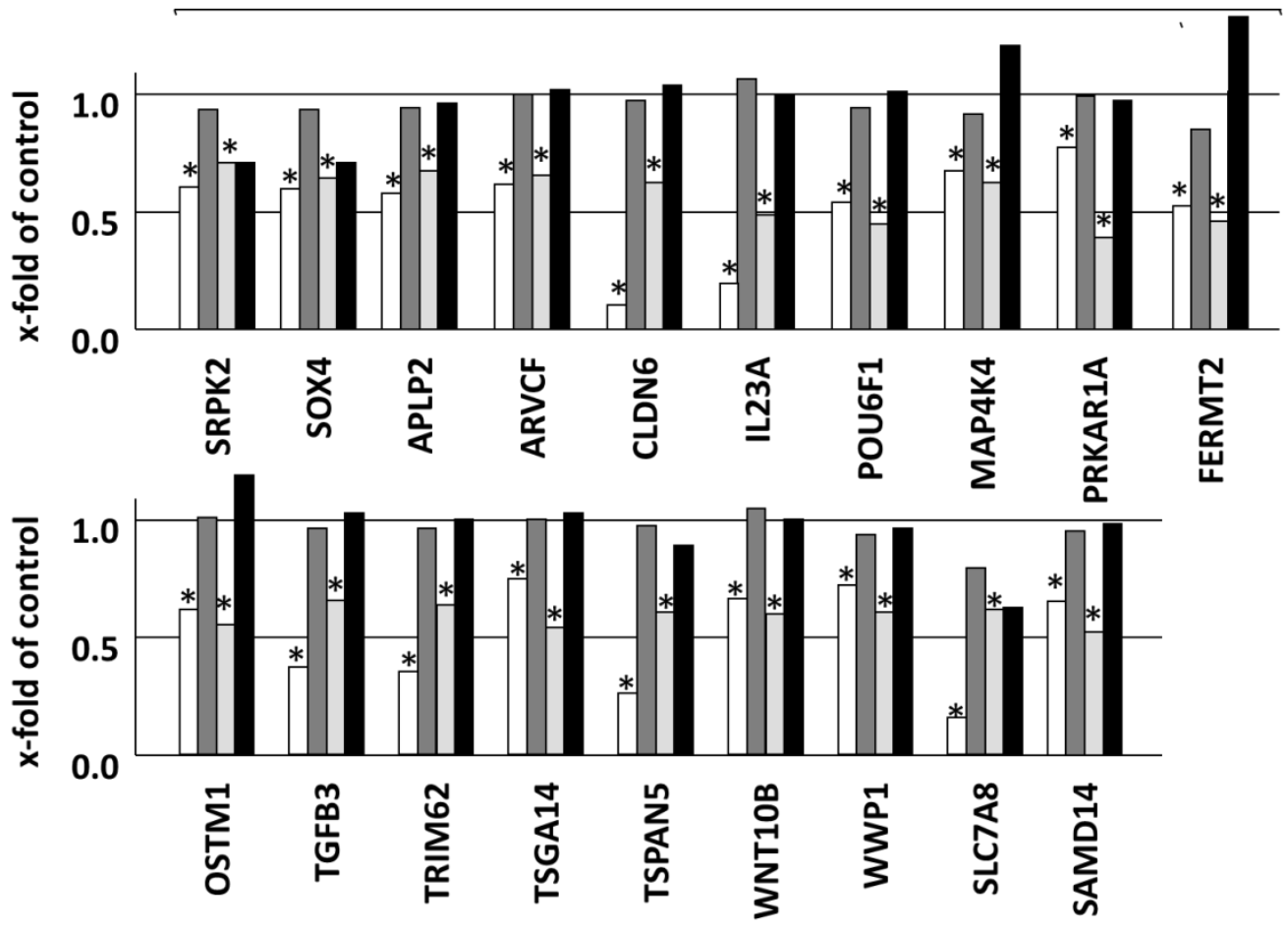

Figure 26: Comparative gene expression profiles from VilCre; $\mathrm{BCL} 9^{-/} / \mathrm{BCL9-2}{ }^{-/}$and microarrays from siRNA treated SW480 cells identified new target genes of the BCL9 proteins

Comparative gene expression profiles from tumors of VilCre; $\mathrm{BCL}^{-\%} / \mathrm{BCL} 9-2^{--}$and SW480 cells treated with siRNA targeting $B$-catenin, $B C L 9$ and $B C L 9-2$, as indicated. The graphs show the log fold change (logFC) of target genes of (A) BCL9, BCL9-2 and B-catenin, (B) BCL9-2 and B-catenin, (C) BCL9 and (D) BCL9-2 as \% of control siRNA-treated cells and tumors of non-transgenic mice, respectively. Asterisks indicate a fold discovery rate (FDR $<0.05)$.

In summary, based on the expression profiles obtained from our microarray analyses in SW480 cells, the impact of BCL9-2 knockout in $\mathrm{BCL} 9^{-/} / \mathrm{BCL9}-2^{-/-}$mice appeared to be more severe in comparison to BCL9 knockout. Moreover, ablation of BCL9-2 had a dramatic influence on gene expression in the induced tumors. Remarkably, this influence was not observed after 
knockdown of ß-catenin in SW480 cells. This indicates a partially ß-catenin-independent influence of BCL9-2 on gene transcription in colon cancer.

Comparison of the differentially regulated genes from our microarray analyses in SW40 cells to those from the tumors derived from BCL9/BCL9-2 knockout mice, we found the HOX gene SOX4 which appeared in both analyses. Moreover, Deka et al. identified SOX6 and PROX1 as putative target genes of the BCL9 protein in vivo. An additional HOX gene, SOX9, was present in the BCL9-2 mediated gene set in SW480 cells.

However, determination of differentially regulated genes in $\mathrm{BCL} 9^{-1-} / \mathrm{BCL} 9-2^{-1-}$ mice does not dissect the dependency on BCL9 or BCL9-2, since the arrays were performed from double mutant animals. To analyze the dependency of the HOX genes in colon cancer cells on BCL9-2 or/and BCL9 in comparison to ß-catenin, qPCRs were performed following knockdown of the proteins in SW480 cells (Figure 27A and B).

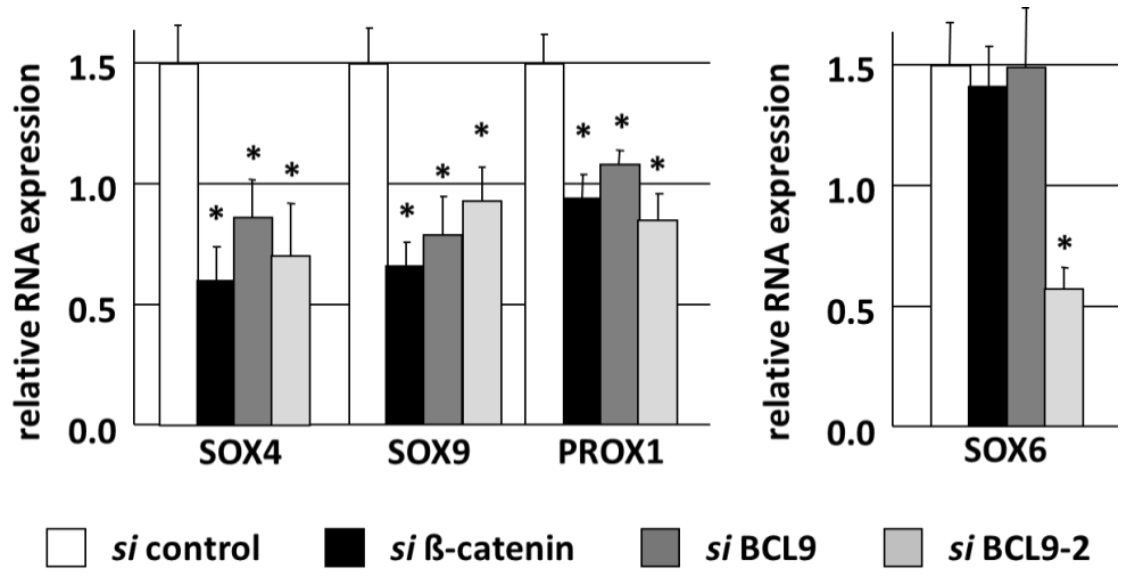

Figure 27: BCL9-2 regulates the expression of HOX genes

Expression of the indicated genes was determined following knockdown of B-catenin, BCL9 and BCL9-2 for $48 \mathrm{~h}$ relative to control siRNA-treated SW480 cells. Quantitative real time PCRs show the mean of least 3 independent experiments and their respective standard deviation $\left({ }^{*} p \leq 0.05\right)$.

The mRNA expression of the HOX genes SOX4, SOX9 and PROX1 in SW480 cells was strongly down-regulated after knockdown of all three proteins. In contrast, SOX6 expression was only regulated by BCL9-2, but not by BCL9 or ß-catenin.

In summary, BCL9-2 transcriptionally regulates different genes of the HOX gene family, which are implicated in cancer progression (Petrova et al., 2002), and which might further trigger the oncogenic potential of BCL9-2 during intestinal tumorigenesis. 
4.10.2 Cyclopholin A (СурА) and the stem-cell-derived neural stem/progenitor cell supporting factor (SDNSF) are newly identified BCL9-2 core target genes in cancer cell lines

Venn diagrams were used to compare all genes that were significantly down-regulated $\geq 1.5$ fold in MCF7, SW480, DLD1 and HCT116 cancer cells. The number of BCL9-2 regulated genes correlated well with the expression level of BCL9-2 in the cell lines (Figure 16 and Figure 28). BCL9-2 knockdown in SW480 showed the strongest effects with 605 affected genes. In contrast, BCL9-2 knockdown was less effective with 18 transcriptional reduced genes in HCT116 cells, with low BCL9-2 protein levels (Figure 28). Moreover, these analyses identified two novel core target genes which were only regulated by BCL9-2 in all analyzed cancer cell lines (Figure 28): Cyclophilin A (СурA) and stem-cell-derived neural stem/progenitor cell supporting factor (SDNSF).

DLD1

HCT116

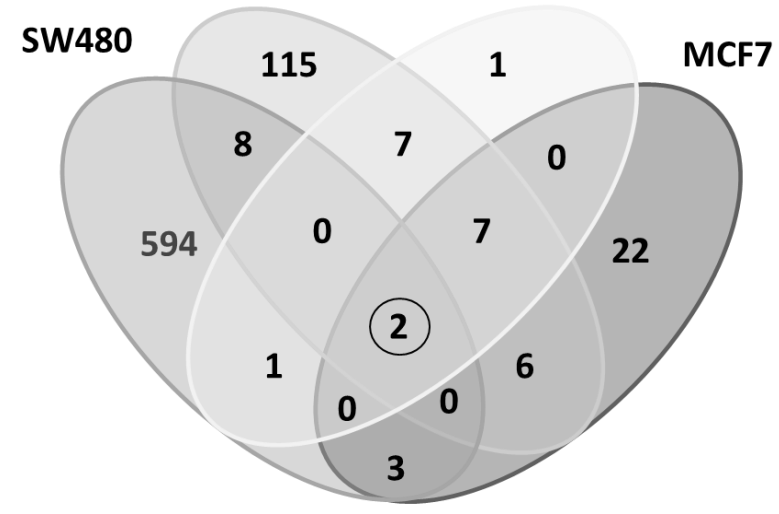

Figure 28: Microarray analysis identified 2 genes as new BCL9-2 core target genes in cancer cell lines Venn diagram showing the overlap of 1.5 fold down-regulated genes upon BCL9-2 knock-down in various cancer cell lines, as indicated $(p \leq 0.05)$.

Quantitative real time PCRs and Western Blot analyses were performed, to validate the dependency of CypA and SDNSF on BCL9-2. Only knockdown of BCL9-2 resulted in strong reduction of mRNA levels of the new core target genes in all tested cell lines (Figure 29A, B and C). Accordingly, protein levels of CypA were reduced after treatment of SW480 and HCT116 cells for $72 \mathrm{~h}$ with siRNA targeting BCL9-2 (Figure 30A, upper panel). B-catenin and BCL9 knockdown had no effect on CypA and SDNSF expression (Figure 29A). 
A.

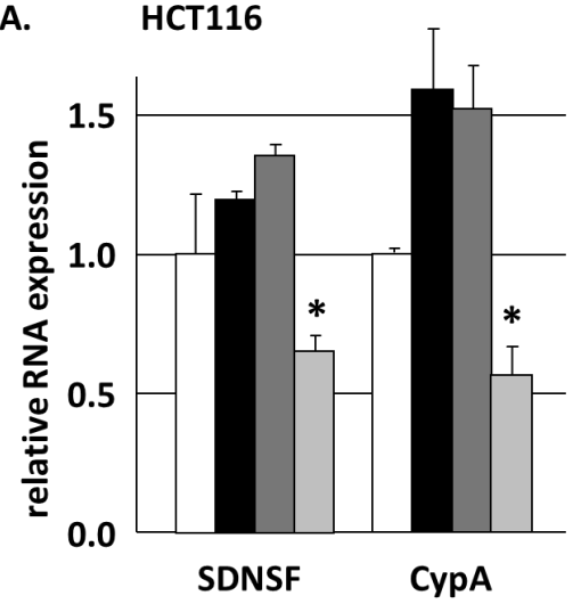

B. $\quad$ SW480

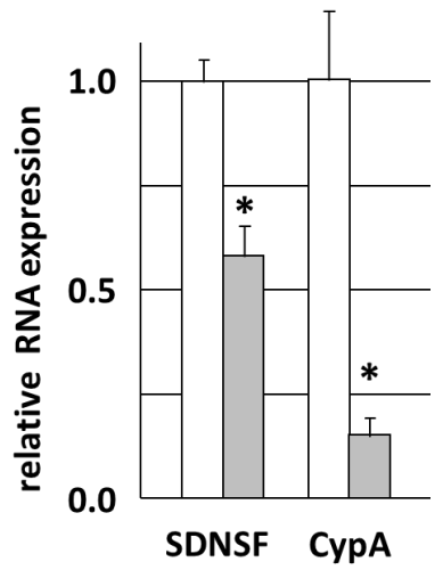

C. $\quad$ MCF7

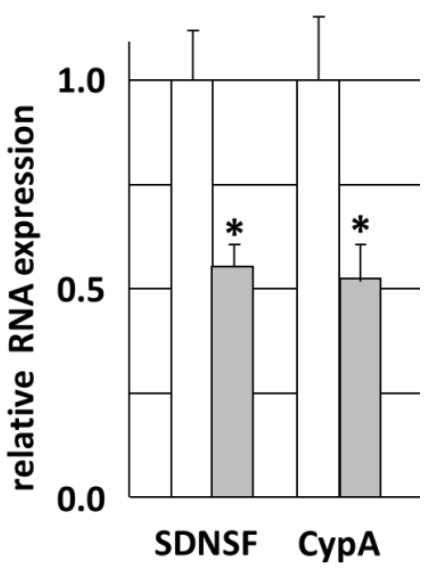

\section{si control}

si ß-catenin

si BCL9

si BCL9-2

Figure 29: BCL9-2 but not BCL9 and B-catenin regulates the mRNA and protein expression of Cyclophilin A (СурА) and stem-cell-derived neural stem/progenitor cell supporting factor (SDNSF) in cancer cell lines

(A, B and C) qRT-PCR determined mRNA levels of SDNSF and CypA following knockdown of B-catenin, BCL9 and BCL9-2 for $48 \mathrm{~h}$ in different cancer cell lines, as indicated. Graphs show the mRNA expression as $\%$ of control siRNA treated cells $\left({ }^{*} p \leq 0.05\right)$.

In summary, BCL9-2 regulates a subset of core target genes in colon cancer cells which might trigger the oncogenic role of BCL9-2 independent of B-catenin. First, Cyclophilin A, which has already been shown to play a role in different tumor entities, might also mediate the oncogenic role of BCL9-2 (Obchoei et al., 2009). Second, SDNSF, which regulates cell-survival (Toda et al., 2003) could also be implicated in BCL9-2 induced tumorigenesis. 


\subsection{Dissecting the ß-catenin-dependent and -independent functions of BCL9-2}

Dissection of the ß-catenin-dependent and -independent function of BCL9-2 is important to characterize the molecular mechanisms for the role of this co-factor for the control of specific target genes. BCL9-2 itself does not contain a classical DNA-binding domain, but might act a cofactor for several transcription factors besides ß-catenin/LEF/TCFs. Therefore, we analyzed the promoters of BCL9-2 regulated genes to identify putative BCL9-2 responsive elements and to characterize a nuclear binding partner of BCL9-2. In this regard, we analyzed the transcriptional regulation of the promoters of the 3 -catenin/BCL9-2 target gene CDX1 and of the BCL9-2 target gene CDX2.

\subsubsection{BCL9-2 regulates the endogenous expression of caudal type homeobox 1 and 2 (CDX1/2)}

The homeodomain transcription factor caudal type homeobox 1 and 2 proteins (CDX1 and CDX2) have been previously identified as Wnt/B-catenin target genes during embryonic intestinal development in mice (Hryniuk et al., 2012;Lickert et al., 2000;Lickert et al., 2002). However, it was shown, that CDX2 expression is not activated by canonical Wnt-signaling after E10.0 in mice (Gao et al., 2009). Moreover, CDX1, but not CDX2, is a well characterized Wnt/ßcatenin target gene in the adult intestine (Hryniuk et al., 2012).

We have analyzed whether BCL9, BCL9-2 and B-catenin regulate the expression of these two homeodomain transcription factors, and performed qPCRs were performed after treatment of SW480 cells with siRNA for 48h targeting BCL9, BCL9-2 and ß-catenin. BCL9 knockdown had no influence on CDX1 and CDX2 expression. In contrast, CDX1 mRNA was reduced after knockdown of ß-catenin and BCL9-2 in SW480 cells. Remarkably, CDX2 was only affected by BCL9-2, but not by ß-catenin knockdown (Figure 30B). Accordingly, BCL9-2 knockdown for 72h reduced the CDX2 protein level in HCT116 and SW480 cells as analyzes by Western Blot analysis (Figure 30A). 
A.
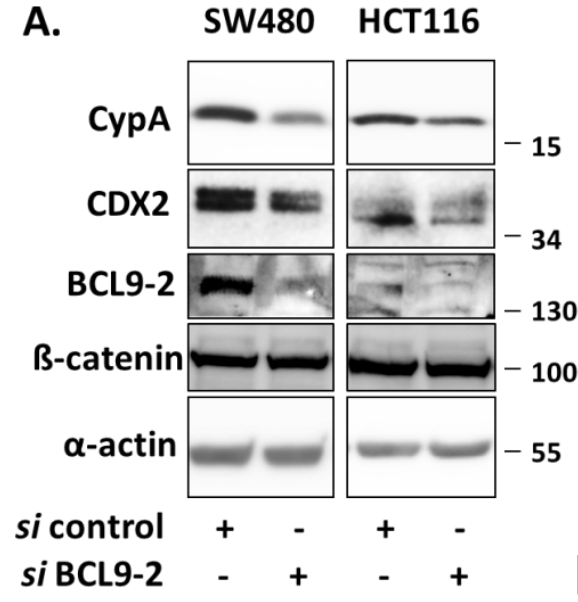

B. SW480

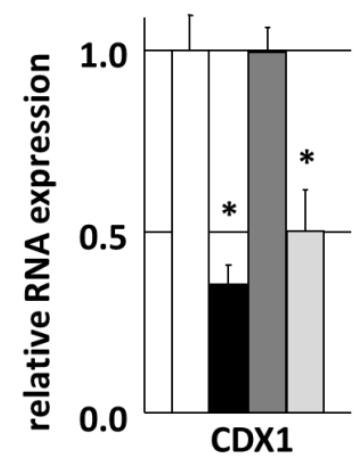

si control si ß-catenin

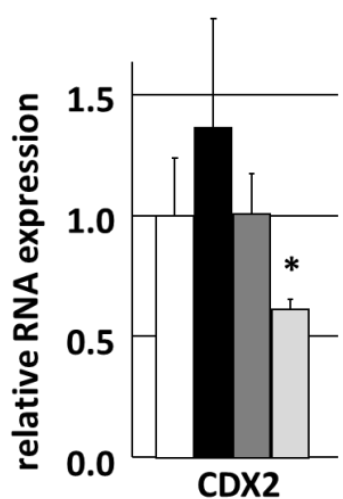

si BCL9 si BCL9-2

Figure 30: The Wnt/ß-catenin target gene CDX1 and the ß-catenin-independent gene CDX2 are regulated by $B C L 9-2$ in colon cancer cell lines

(A) $30 \mu \mathrm{g}$ of nuclear protein lysates were used in Western Blot analysis to determine CDX2 and CypA protein expression following knockdown of BCL9-2 for 72h (second panel) in SW480 and HCT116 cells. $\beta$ actin (bottom panel) and $ß$-catenin (third panel) served as controls for equal protein loading. (B) qPCR of CDX1 and CDX2 following knockdown of ß-catenin, BCL9 and BCL9-2 in cancer cell lines, as indicated. Graphs show the mRNA expression relative to control siRNA treated cells $\left({ }^{*} p \leq 0.05\right)$.

In summary, BCL9-2 regulates the endogenous expression of CDX1 and CDX2, which had been shown to be up-regulated during early stages of tumor development (Bakaris et al., 2008; Phillips et al., 2003). Remarkably, only BCL9-2, but not ß-catenin or BCL9, regulates the mRNA and protein expression of CDX2 in colon cancer cell lines. In contrast, endogenous expression of CDX1 is dependent on B-catenin and BCL9-2.

\subsubsection{Transcriptional activation by the proximal promoters of the homeodomain} transcription factors CDX1 and CDX2 requires BCL9-2, but not $B$-catenin in colon cancer cell lines

To identify a promoter region, which is responsible for BCL9-2 mediated transcriptional activation, Luciferase assays were performed in SW480 and HCT116 colon cancer cell lines following knockdown of B-catenin and BCL9-2. To this end, Luciferase-reporter constructs, containing different length of the proximal promoter of the human CDX1 (Suh et al., 2002) and CDX2 were used. All Luciferase constructs contained different fragments of the upstream CDX1 and CDX2 promoter regions and $+73 \mathrm{bp}$ of the CDX1- and $+76 \mathrm{bp}$ of the CDX2-3'UTR, respectively, relative to the transcription start site (Figure 31and Figure 32). 
A.
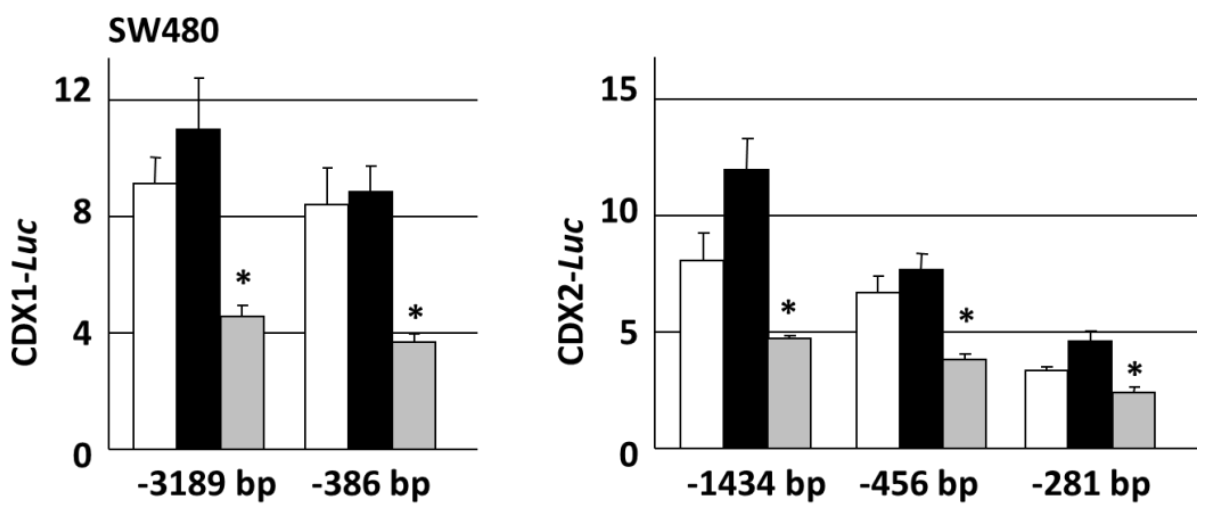

B.
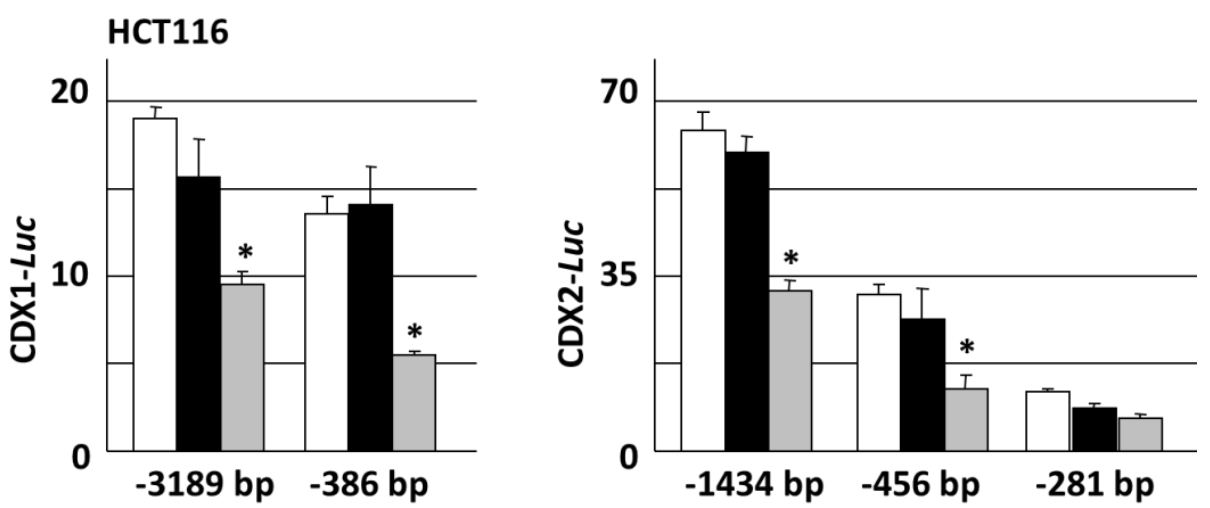

si control

si ß-catenin

si BCL9-2

Figure 31: BCL9-2, but not B-catenin knockdown reduces the activity of CDX1 and CDX2 Luciferasereporters

For Luciferase assays (A) SW480 and (B) HCT116 cells were pretreated with siRNA for 48h targeting Bcatenin, or BCL9-2 and non-targeting siRNA, followed by 36h transfection of CDX1 and CDX2 Luciferasereporters containing different length of each of their proximal promoters, as indicated. The graphs show the fold change of empty control plasmids with their respective standard deviation $\left({ }^{*} p \leq 0.05\right)$.

CDX1 and CDX2 promoter driven Luciferase expression was independent of ß-catenin in both colon cancer cell lines, although all CDX1 reporters and the $-1434 \mathrm{bp}$ and $-456 \mathrm{bp}$ CDX2 reporters contain LEF/TCF responsive elements. Compared to the control, knockdown of BCL92 reduced the activity of the $-3189 b p$ and the smaller $-386 b p$ CDX1 Luciferase-reporter to $50 \%$ (Figure 31A and B, left). Moreover, knockdown of ß-catenin even slightly induced all CDX2 Luciferase-reporter in SW480 (Figure 31A, right). This tendency was already observed on CDX2 mRNA level after knockdown of B-catenin in qPCR (Figure 30A and B). BCL9-2 knockdown significantly inhibited the activities of the $-1434 \mathrm{bp}$ and the $-456 \mathrm{bp}$ CDX2 reporters in SW480 and HCT116. The $-281 \mathrm{bp}$ proximal promoter fragment of CDX2 still responded to BCL9-2 knockdown in both cell lines, but to a lesser extent compared to the larger $-456 \mathrm{bp}$ CDX2 reporters (Figure 31A and B, right). 
In conclusion, $C D X 2$ and the Wnt/B-catenin target gene CDX1 is regulated by BCL9-2 through a responsive element in the proximal promoter of $-386 /+73 b p$ CDX1 and of $-456 /+76 b p$ CDX2. Moreover, both CDX reporters were independent of ß-catenin indicating that the LEF/TCF binding elements (Wnt responsive elements - WRE) in the proximal promoters of the CDX genes are not responsive to ß-catenin in colon cancer cell lines.

\subsubsection{Identification of putative transcription factor binding sites in the proximal promoters of CDX1 and CDX2}

The promoters of $\mathrm{CDX} 1$ and $\mathrm{CDX} 2$ were further analyzed in more detail, to evaluate the BCL9-2 mediated transcriptional regulation of the ß-catenin/BCL9-2 target gene CDX1 compared to the BCL9-2 target gene CDX2. TRANSFAC ${ }^{\circledR}$ MATCH/PATCH (Transfac Professional 2008.4 (Kel et al., 2003; Matys et al., 2003)) was used to identify putative transcription factor binding elements (BE), which were present in the $-386 /+73 b p$ CDX1 and the $-456 /+76 b p$ CDX2 promoter. TRANSFAC ${ }^{\circledR}$ MATCH identifies predicted transcription factor binding elements by using a library of positional weight matrices, while TRANSFAC ${ }^{\circledR}$ PATCH identifies pattern-based transcription factors. Both applications use a set of binding sites provided from TRANSFAC ${ }^{\circledR}$ Public 6.0 (http://www.gene-regulation.com/pub/databases.htm/\#transfac).

TRANSFAC $^{\circledR}$ MATCH analyses identified various putative binding elements (BEs) in both proximal promoters: Binding elements for LEF/TCF, OCT4 (octamer-binding transcription factor 4), NFkB (nuclear factor kappa-light-chain-enhancer of activated B cells), BCL6 (B-Cell Lymphoma 6 protein) and also for CDX2 were present in both promoters (Figure 32A and B).

Of note, CDX2 itself was shown to bind to the TATA box of the CDX1 promoter and thereby activate CDX1 expression (Mutoh et al., 2009). The two LEF/TCF binding sites in the CDX1 promoter were previously described as functional elements in early mouse embryonic intestines (Lickert et al., 2000;Lickert et al., 2002). In addition, the CDX2 promoter contains a previously identified SMAD-binding element, which is activated by BMP2/4 in intestinal metaplasia (Barros et al., 2008). An second SMAD-binding element was identified in the proximal promoter of the CDX2 gene close to the transcription start site by TRANSFAC $^{\circledR}$ MATCH (Figure 32B).

Moreover, both promoters harbor multiple SP1-binding elements (Specificity Protein 1), which are very common in GC-rich promoters. TRANSFAC ${ }^{\circledR}$ MATCH identified two SP1-binding elements in the proximal promoter of the CDX1 gene. In the TATA-less promoter of CDX2 SP1 
binding elements (BEs) were randomly distributed as identified by TRANSFAC ${ }^{\circledR}$ PATCH. In addition we found three suboptimal SP1-BEs the CDX2 proximal promoter (Figure 32A and B).

To investigate whether the identified TF-binding elements are conserved between Mus musculus and Homo sapiens, T-COFFEE ${ }^{\circledR}$ was used (Di et al., 2011;Notredame et al., 2000). This tool allows the combination of multiple sequence alignment including the determination of the degree of conservation between sequences. To this end, the proximal promoter sequences of human and mouse CDX1 and CDX2 were analyzed (Figure 32A and B). The proximal promoter of the CDX1 gene showed overall less conservation compared to that of CDX2. Previously identified transcription factors binding sites for LEF, OCT4 and CDX2 in the -386bp CDX1 and 456bp CDX2 promoter were highly conserved in humans and mice. Both SMAD-binding elements were highly conserved in mouse and human CDX2 promoters and a conserved NFKBbinding element was present in the proximal promoter of CDX1 while only human species harbor this element in the CDX2 promoter. BCL6-binding elements were found in both CDX promoters of Homo sapiens, but not in the proximal promoter of rodent CDX1, suggesting a less important role of BCL6. However, SP1-binding elements were present in all promoters of the CDX genes, but only the very proximal SP1-elements located very close to the transcription start were conserved in the promoters of both species. In contrast, the more distal SP1-binding elements were only present in the human CDX1 and CDX2 promoters (Figure 32B).

Thus, multiple similar binding elements were present in the proximal promoters of CDX1 and CDX2 which are highly conserved between human and mouse including the binding sites for BCL6, NFKB, OCT4, CDX2, LEF/TCF and SP1 transcription factors. 


\section{A. proximal pomoter of CDX1}

BCL6

Homo sapiens -386bp GTGAAGTTGG CCTAGAATCCCCCGGCCCCTGGGAG-CTTGT- . . . - TCCT-CCGCCTGTAAAAT-GGGGCTGC Mus musculus $-405 \mathrm{bp}$ TGACAGTGTCCCCATGCTCTGTG-GGCGGTGTGAGGCTCGCCTAGGGTCATGCCACCACTCCACCCCGCTCCTCG

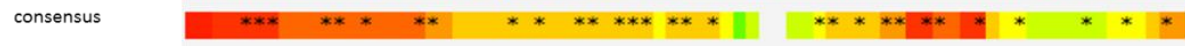

Homosapiens -320bp GGGCCG - . - - - TCCACGCGGCCACC-GGAAGGACAAGGTGTTCAGGCCGCTAGGCCGCTCCCTGGCAAGCGATTC Mus musculus $333 \mathrm{bp}$ GAGCCAGTITCTCCACCTG-TAACCCAGGGGTGGGTGGTGGGGAGGTCCCTGCG--ACCCCCGAGGAG-GAGTC \begin{tabular}{l|lllllllllll|l} 
consensus & $* * * *$ & $* * * * *$ & $*$ & $* * *$ & $*$ & $*$ & $* * * *$ & $* * * * * * *$ & $*$ & $* * * * *$ & $* * * * * *$
\end{tabular} SP1

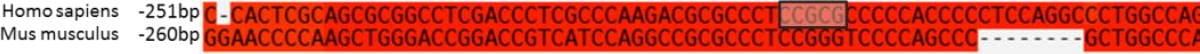

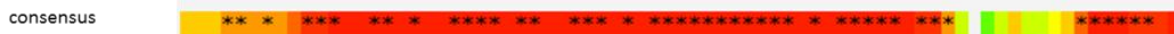

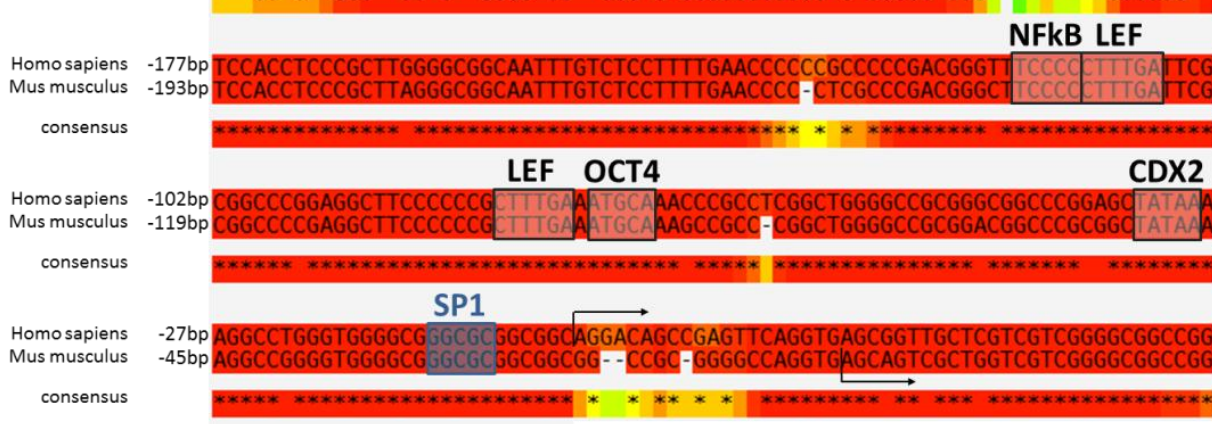

Mus musculus +29bp CCGGGCGCGGCGGCTCCAGGGCCCAGC

consensus $\quad * \quad * * * * * * * * * * * * * * * * * * * * * 1$

\begin{tabular}{|c|}
\hline MATCH \\
\hline PATCH \\
\hline
\end{tabular}

TF-BE identified by TRANSFAC ${ }^{\oplus}$

\section{B. proximal promoter of CDX2}

proximal SP1-BE close to the TS

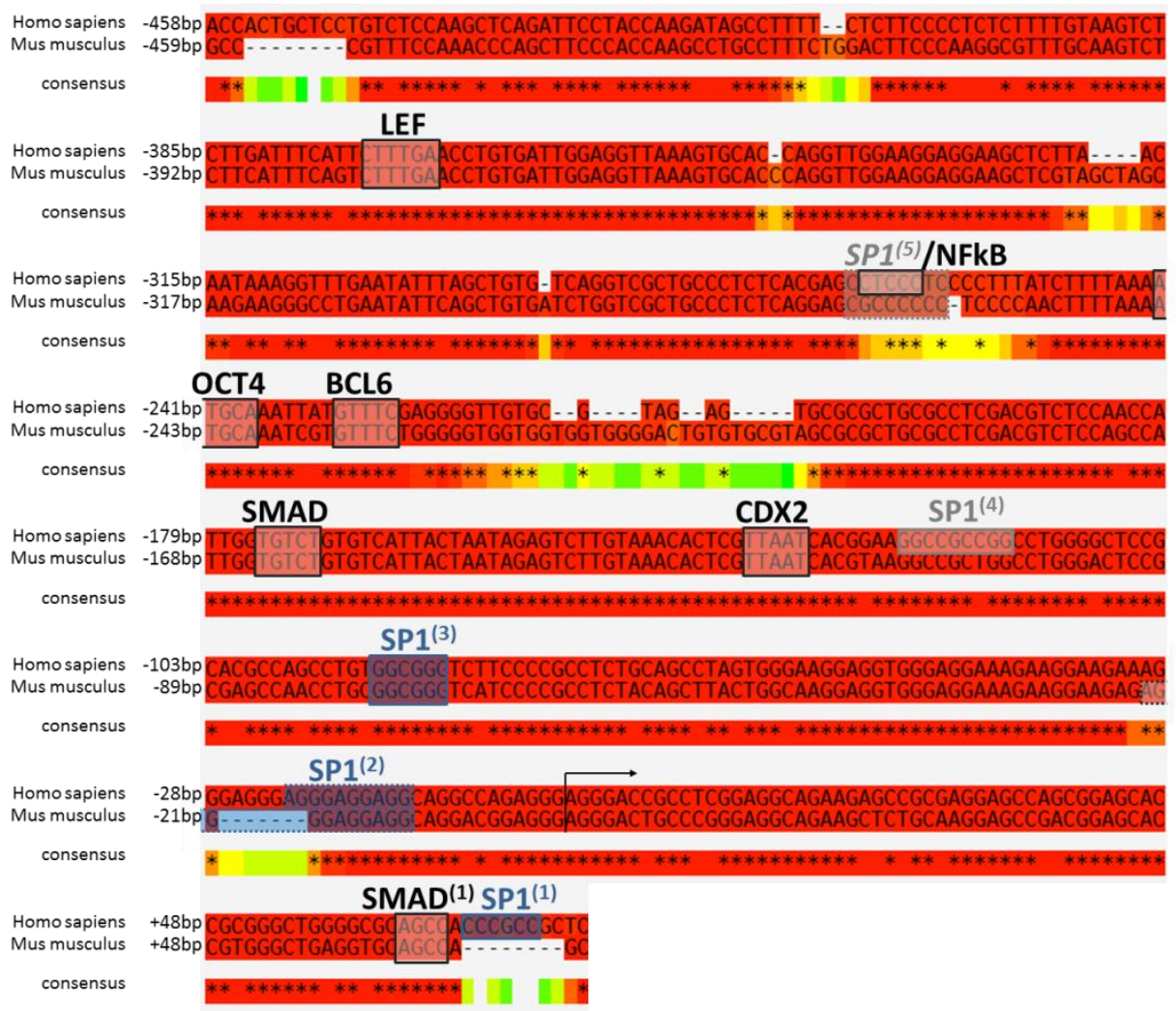

Figure 32: Transcription factor binding elements in the CDX1 and CDX2 proximal promoter Proximal promoters from Homo sapiens, Mus musculus and Rattus norwegicus (A) of CDX1 and (B) CDX2 including trough TRANSFAC ${ }^{\circledR}$ identified putative transcription factor binding elements (TF-BE) on the indicated position from the transcription start (TS). The degree of conservation between the organisms is indicated by a color code. 


\subsubsection{BCL9-2 modulates CDX1 and CDX2 reporter activity by Specificity Protein 1 (SP1)-binding elements in their proximal promoter in colon cancer cell lines}

To identify a binding site which is responsible for BCL9-2 transcriptional regulation, all binding elements as identified above (see 4.11.3) were mutated using mutagenesis PCR to disrupt the binding of the respective transcription factors. The activities of the mutated Luciferasereporters were analyzed in SW480 and HCT116 which were pretreated for 48h with siRNA targeting BCL9-2 and compared to control siRNA treated cells.

A) Transcriptional regulation of CDX1 (Figure 33)

In general, mutation of all TF-binding elements in the promoter of CDX1 only slightly reduced the reporter activities in SW480 cells. In HCT116 mutation of the binding motif for LEF, NFKB, OCT4 and SP1 led to a reduction of reporter activities of approximately $45-60 \%$. However, each of the mutated reporters was still expressed with well detectable levels (Figure 33).

Remarkably, single and double LEF mutated CDX1 Luciferase-reporters still responded to knockdown of BCL9-2 in SW480 and HCT116 cells (Figure 33), indicating that BCL9-2 regulated their expression independently of these LEF-binding elements.

Also the mutations of the BCL6-, NFKB- or OCT4-binding elements still showed a reduced CDX1 reporter activity following BCL9-2 knockdown (Figure 33).

Mutation of the CDX2-binding element in the proximal CDX1 promoter (Figure 32A) was not investigated since this binding site is located close to the transcription start site and mutation will lead to a complete loss of reporter activity (Mutoh et al., 2009).

The distal SP1-binding element mutant still responded to BCL9-2 knockdown. However, mutation of the very proximal SP1-binding element lost the response to knock down of BCL9-2. Similarly, CDX1 reporter containing SP1-binding element-double mutations also did not respond to BCL9-2 knock down (Figure 32A and Figure 33).

These data demonstrate that BCL9-2 regulates CDX1 gene transcription through a newly identified SP1 binding element in the proximal promoter region close to the transcription start site. 

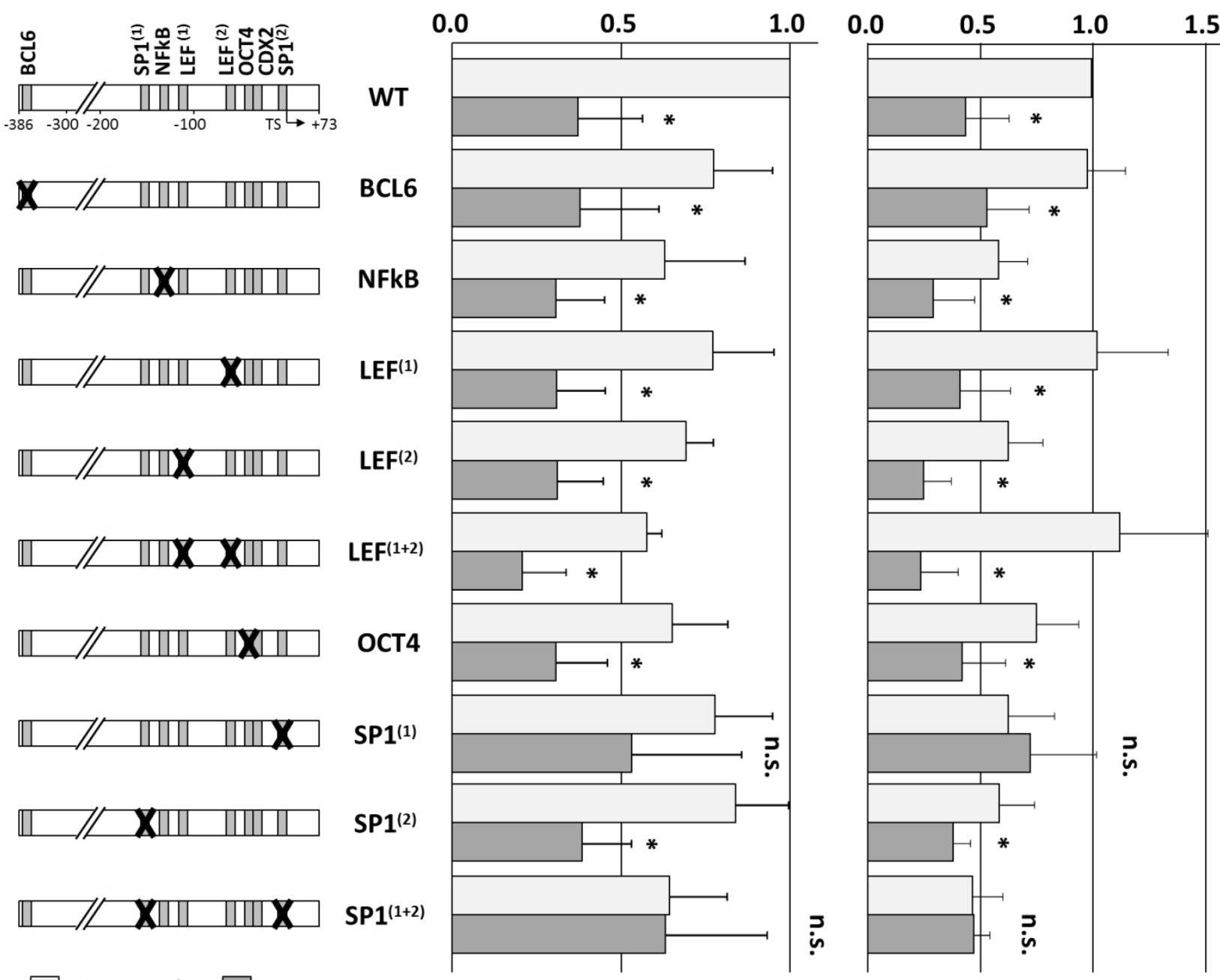

si control $\square$ si BCL9-2

SW480

HCT116

Figure 33: BCL9-2 regulates CDX1 reporter gene expression by an SP1 responsive element in the proximal promoter

For Luciferase assays SW480 and HCT116 cells were pretreated with siRNA targeting BCL9-2 and nontargeting siRNA for $48 \mathrm{~h}$, followed by $36 \mathrm{~h}$ transfection of $-386 \mathrm{bp} /+73 \mathrm{bp}$ CDX1 Luciferase-reporters containing different mutations of TF-responsive elements, as indicated (left). The graphs show the relative Luciferase-reporter activity to the CDX1-Luciferase wild-type (WT) activity and their standard deviation. Significances were calculated to the respective control siRNA treated cells $\left({ }^{*} p \leq 0.05\right)$.

\section{B) Transcriptional regulation of CDX2 (Figure 34)}

Mutation of LEF-, BCL6- and CDX2-binding elements had just minor effects on the -456bp CDX2 Luciferase-reporter activity in HCT116 and SW480 cells Figure 34). In SW480 cells, only mutation of the SMAD- and NFKB-binding elements decreased the activity to a greater extent to approximately $50-60 \%$. In HCT116 cells, mutation of NFKB- and the distal SMAD-binding elements reduced the $-456 \mathrm{bp}$ CDX2 Luciferase-reporter activity to approximately $40-70 \%$. However, each of the mutated $-456 b p$ CDX2 Luciferase-reporters was still expressed with well detectable levels in SW480 and HCT116 cells (Figure 34). 
Similar to our results for the CDX1 promoter, mutation of the LEF-binding element in the CDX2 reporter also had no influence on the repressive function of BCL9-2 knockdown. In addition, also mutations of the BCL6-, NFKB- or CDX2-binding elements did not change the capability of BCL9-2 knockdown to further reduce these CDX2 reporter activities (Figure 34).

Interestingly, one of each mutant for the two SMAD-binding elements was potent to inhibit the BCL9-2 effect in either, HCT116 or in SW480 cells, respectively (Figure 34). This indicates that the SMAD pathway is efficient to modulate CDX2 reporter activity in these cells.

Like many TATA-less genes, the CDX2 promoter harbors multiple putative SP1-binding elements. SP1-MT(2) and -MT(5) are mutated optimal SP1 sites, while SP1-MT(1), -MT(3) and MT(4) are mutations of suboptimal SP1 binding sites (Figure 32B).

In HCT116, the basal activity of the CDX2 reporters containing the mutation of the four proximal SP1-binding elements (MT1-4) was reduced to approximately $60-40 \%$ of the unmutated promoter, while in SW480 mutation of these SP1-binding elements (MT1-4) had no influence on the reporter activities. In addition, the most distal SP1-binding element (MT5) did not affect the basal CDX2 reporter activity in both cell lines. However, double mutation (dMT) of the two optimal SP1-binding elements $(\mathrm{dMT} 2+5)$ strongly reduced the reporter activity in HCT116 and SW480 cells (Figure 34).

In SW480 cells, single mutations of the distal SP1-binding elements (MT4 and 5),' and the proximal SP1-binding element (MT2) still showed significantly reduced reporter activity following BCL9-2 knockdown. In contrast, mutation of two SP1 sites in the proximal promoter (MT1 and 3), completely lost the inhibition of promoter activity after knockdown of BCL9-2 (Figure 34).

Remarkably, all mutated SP1-binding elements (MT1-5) of the CDX2 promoter did not further respond to BCL9-2 knockdown in HCT116 cells. In both cell lines, double mutations of SP1 sites (MT2+5) also did not respond to BCL9-2 knockdown (Figure 34).

These results clearly demonstrate that the BCL9-2 mediated transcriptional regulation of the CDX2 promoter requires the presence of several functional SP1 binding elements in both cell lines. 
$-456 /+76$ bp CDX2 promoter

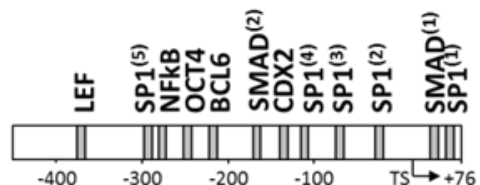

$\mathbf{X} \quad\|\|\|\|\|\|\|\|\|\| \square$

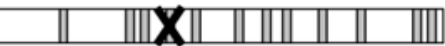
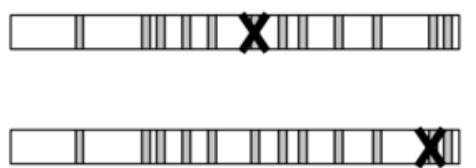

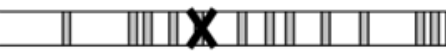

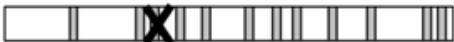

NFkB

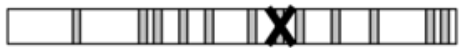

CDX2

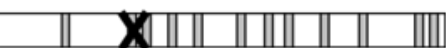

$S P 1^{(5)}$

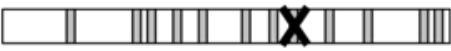

SP1(4)

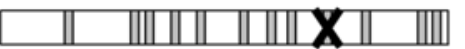

SP1 ${ }^{(3)}$

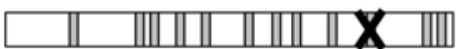

SP1 ${ }^{(2)}$

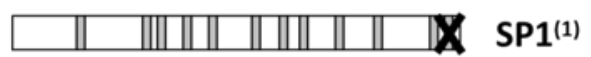

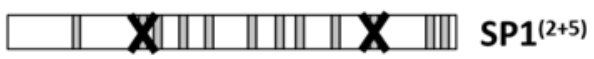

si control $\square$ si BCL9-2 relative $-456 /+76$ bp CDX2-LUC-activity

0.0

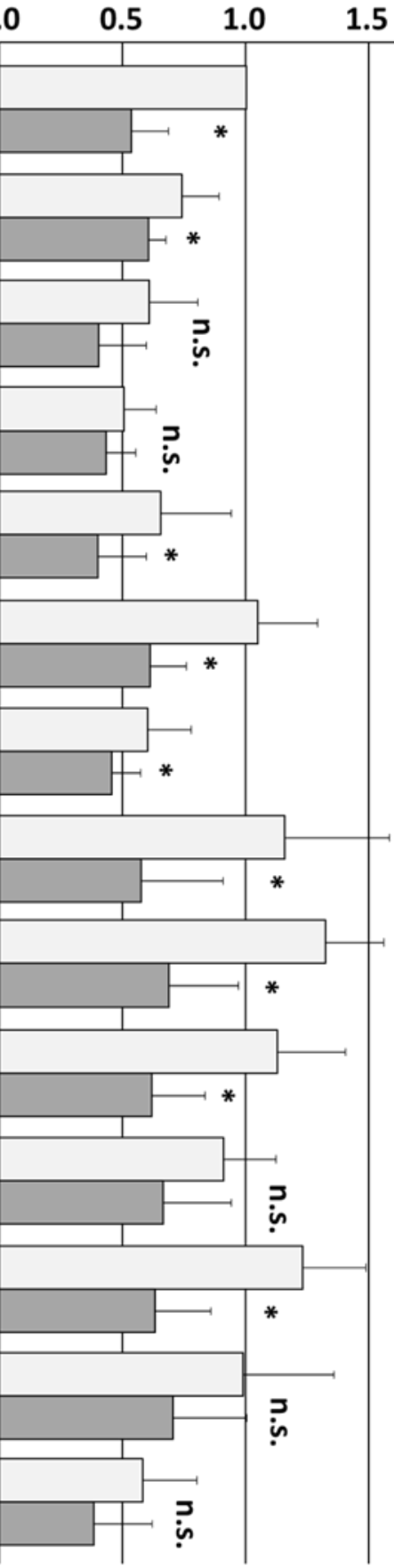

SW480 $\begin{array}{llll}0.0 & 0.5 & 1.0 & 1.5\end{array}$

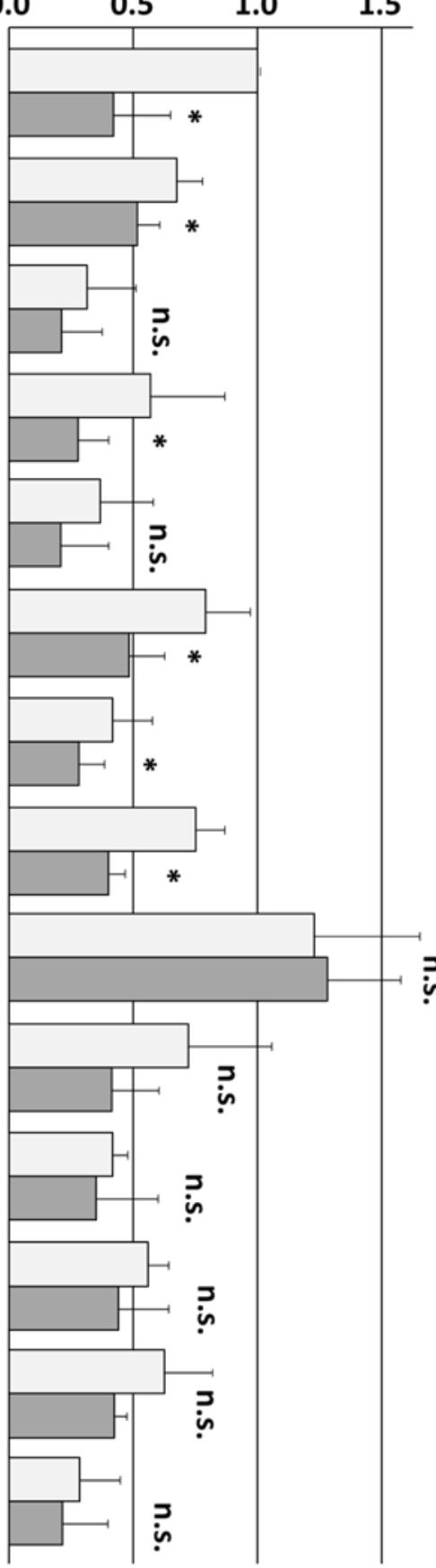

HCT116

Figure 34: SP1 binding elements mediate the BCL9-2 dependent regulation of the CDX2 promoter For Luciferase assays SW480 and HCT116 cells were pretreated with siRNA targeting BCL9-2 and nontargeting siRNA for $48 \mathrm{~h}$, followed by $36 \mathrm{~h}$ transfection of $-456 \mathrm{bp} /+76 \mathrm{pb}$ CDX2 LUC-reporters containing different mutations of TF-BSs, as indicated. The graphs show the relative $L U C$-reporter activity to the CDX2-LUC wild-type (WT) activity and their standard deviation. Significances were calculated to the respective control siRNA treated cells $(* p \leq 0.05)$.

In addition, the CDX2 reporter activity was strongly reduced after mutation of the OCT4binding element, indicating the importance of this element for the transcription of CDX2 in 
colon cancer cell lines. Moreover, BCL9-2 knockdown did not further significantly reduce the OCT4 mutated reporter in HCT116 and SW480 cells (Figure 34).

Because CDX1 and CDX2 reporter activities were dependent on the presence of an OCT4 binding element, OCT4 was overexpressed in SW480 cells to determine whether the reporters are inducible by OCT4 or if the OCT4 binding element generally exhibits some activating function independently from OCT4 protein expression (Figure 35).
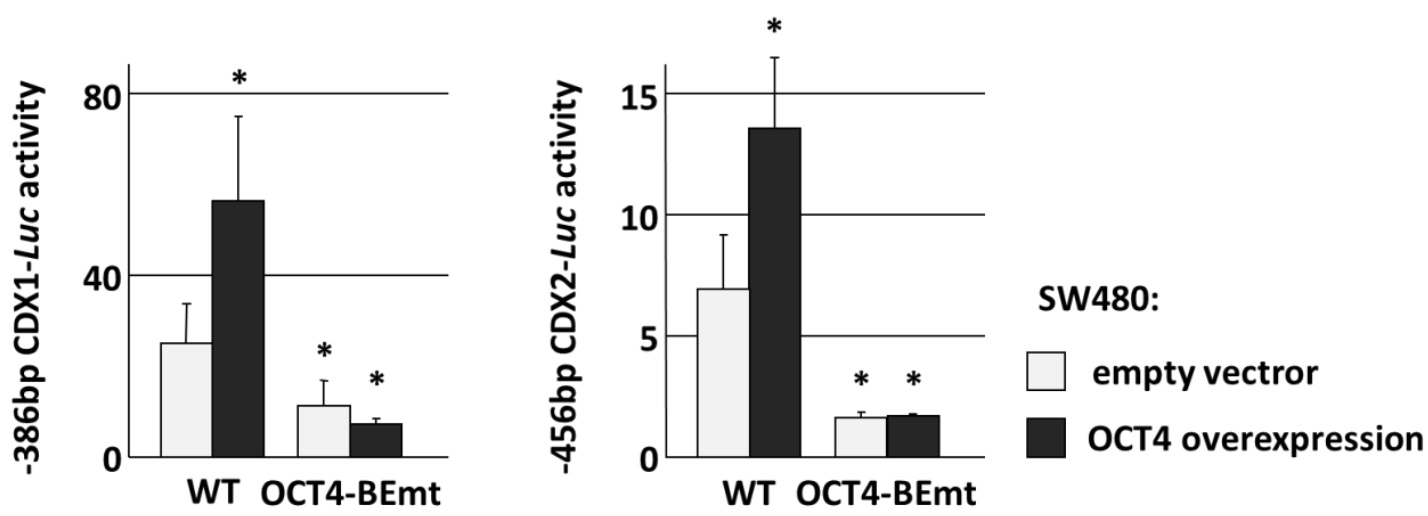

Figure 35: OCT4 induces the CDX1 and CDX2 reporter activities through a newly identified OCT4 binding element

For Luciferase assays SW480 were transfected with 200ng OCT4 overexpression plasmid and $-456 \mathrm{bp} /+76$ pb CDX2 or -386bp/+75bp CDX2 Luciferase-wild-type (WT) or OCT4-binding element mutated (OCT4BEmt) reporters, as indicated. The graphs show the absolute Luciferase-reporter activities and their standard deviation. Significances were calculated to the activity of the respective WT reporters without OCT4 overexpression $\left({ }^{*} p<0.05\right)$.

Interestingly, overexpression of OCT4 resulted in significant induced CDX1 and CDX2 activity of wild-type (WT) reporters in SW480 cells. As shown previously, mutation of the OCT4-binding elements in both promoters led to a significant inhibition of reporter activities. Accordingly, OCT4 overexpression showed no longer any activating effects (Figure 35).

In summary, LEF-binding elements in the proximal promoter of CDX1 and CDX2 are dispensable for BCL9-2 mediated gene transcription, indicating that BCL9-2 acts independently from $ß$-catenin in the regulation of these target genes. However, the proximal promoter of CDX1 and CDX2 contain novel OCT4 responsive elements which mediate the transcriptional activation of the CDX1 and CDX2 reporters.

The most important results of these studies reveal that the cis-regulatory elements of both, the CDX1 and CDX2 gene, harbor multiple SP1-binding elements, which are essential for the activation of the proximal promoters in dependence of BCL9-2. Interestingly, the proximal SP1 binding elements close to the transcription start site are most important for the BCL9-2 mediated CDX1- and CDX2-gene transcription. 


\subsection{BCL9-2 interacts with the transcription factor SP1 in colon cancer cell lines}

BCL9-2 regulated the expression of the CDX1- and CDX2-Luciferase reporters through SP1binding elements in their proximal promoters. To determine whether BCL9-2 regulates gene expression by binding to SP1 and thereby probably to the promoter of target genes, coimmunoprecipitations were performed in HCT116 and SW480 colon cancer cell lines. Specific antibodies (Table 8) were used to precipitate BCL9-2 and SP1, respectively. In addition, Pygopus 2 was precipitated as known interaction partner of BCL9-2, rabbit serum (IgG) served as negative control.

The specific antibody recognizing BCL9-2 only precipitated its targeted protein, but not BCL9 (Figure 36, bottom panel). Moreover, binding to Pygo2 was confirmed by precipitation of BCL9-2 and vice versa (Figure 36, third panel).

IP:

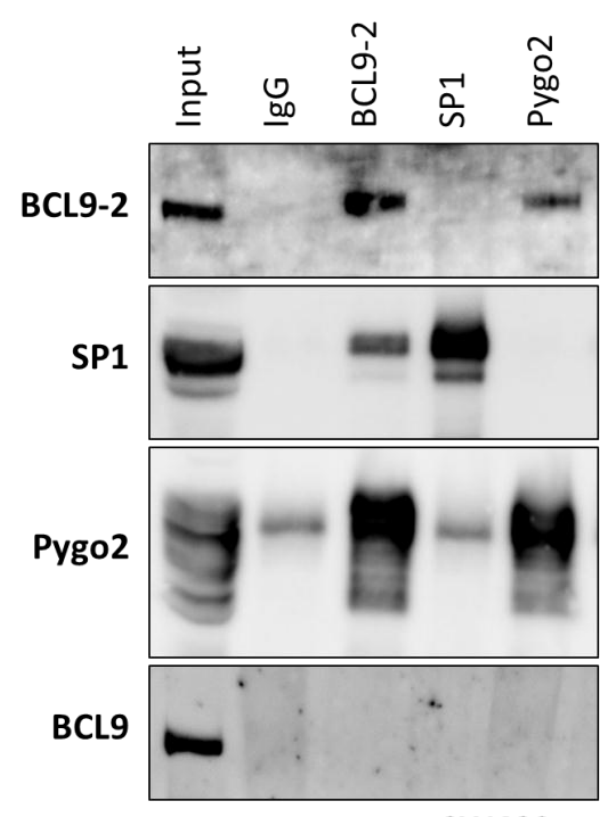

SW480
IP:
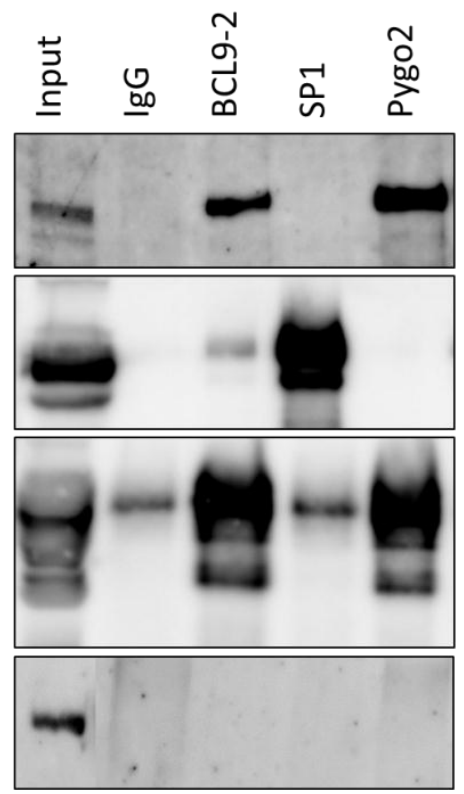

HCT116

Figure 36: Co-Immunoprecipitation identified SP1 as a new interaction partner of BCL9-2

Co-Immunoprecipitations (Co-IPS) were performed from SW480 and HCT116 using specific antibodies, as indicated (IP). IgG rabbit serum served as negative control. $10 \%$ of IPs and $5 \%$ of the respective inputs were used for SDS-PAGE in addition to specific antibodies detecting the indicated proteins/interaction partners (left).

Of particular note, co-Immunoprecipitation identified BCL9-2 as interaction partner of the transcription factor SP1 in SW480 and HCT116 cells. After immunoprecipitation of BCL9-2, SP1 
was identified by Western blotting in the precipitated complexes in both cell lines. The reverse experiment using a SP1-antibody was not successful. HCT116 cells express lower levels of BCL9-2 compared to SW480 cells (Figure 16, upper panel and Figure 36, upper panel). According to this, less SP1 protein was detected in the BCL9-2-IP in HCT116 cells compared to SW480 cells.

In conclusion, the co-immunoprecipitation experiments show that BCL9-2 can be found in a complex not only with Pygopus 2, but also with SP1 in colon cancer cells.

These completely novel results uncover a mechanism which explains the transcriptional regulation of BCL9-2 target genes which are ß-catenin-dependent and -independent. 


\section{Discussion}

The role of BCL9/Legless in Drosophila has been studied intensively (Kessler et al., 2009;Kramps et al., 2002;Townsley et al., 2004). BCL9/Legless was shown to be essential for canonical Wnt signaling during embryogenesis in Drosophila (Kramps et al., 2002). In this context BCL9/Legless mediates the recruitment of the segment polarity gene Pygopus which recruits and modulates components of the chromatin remodeling machinery (Chen et al., 2010;Fiedler et al., 2008;Kessler et al., 2009;Nakamura et al., 2007). The function of the vertebrate BCL9 proteins was shown to be primarily context-dependent (Brembeck et al., 2006; Brembeck et al., 2004;Sustmann et al., 2008). However, the role of the vertebrate BCL9 and BCL9-2 seems to be more complex than in Drosophila. BCL9-2 double knockout mice are early embryonic lethal (Matsuura et al., 2011) while conditional ablation of BCL9/BCL9-2 in the intestine caused no obvious phenotype (Deka et al., 2010). This indicates that the BCL9 proteins are not necessary for normal homeostasis while at least BCL9-2 exerts important functions during embryonic development. BCL9-2 is overexpressed in cancers (Zatula and Brembeck, unpublished)(Adachi et al. 2004; Deka et al. 2010; Sakamoto et al. 2007; Toya et al. 2007b). These findings provide further evidences for BCL9-2 to act as a proto-oncogene in colon tumorigenesis.

However, a complete investigation of the BCL9 proteins and their function in normal intestines and during the multistep model of carcinogenesis was still missing. Here, their expression and transcriptional function in normal and tumorigenic epithelia in mice and humans was examined. Moreover, for the first time, this study provides evidence for a ß-catenin independent function of BCL9-2 and defines a novel mechanism how BCL9-2 might act as transcriptional co-activator independently of ß-catenin.

\subsection{The role of BCL9 and BCL9-2 in intestinal homeostasis}

The Wnt/ß-catenin pathway is crucial for intestinal homeostasis and diverse developmental processes such as specification of the mesoderm and neuroectoderm, body axis formation and intestinal specification during embryogenesis (Gadue et al., 2006;Kemp et al., 2005;Mohamed et al., 2004;Nostro et al., 2008;Sherwood et al., 2011), as well as intestinal homeostasis in the adult organism (Pinto et al., 2003). In addition, BCL9 and BCL9-2 have been shown to be essential for different ß-catenin-dependent developmental processes such as mesoderm 
patterning in zebrafish, myogenic differentiation and for proper placenta development in mice (Brack et al., 2009;Brembeck et al., 2004;Matsuura et al., 2011), while it appears, that other processes do not require the participation of the BCL9 proteins, like the formation of the dorsal organizer or the posteriorization of the anterior neuroectoderm (Brembeck et al., 2004).

We addressed the question whether BCL9 and BCL9-2 participate in the Wnt/B-catenin signaling-dependent maintenance of intestinal homeostasis. To this end, we characterized the expression of BCL9 and BCL9-2 in intestinal tissues of mice using specific antibodies (Brembeck et al., 2011). BCL9 protein expression was found in all intestinal cell types including the crypts. Canonical Wnt signaling in the intestinal crypt compartment and the subsequent expression of ß-catenin target genes is required for the maintenance of the proliferative capacity of intestinal stem cells (Korinek et al., 1998a). Our results showed that intestinal ß-catenin target genes such as TCF1, EPHB2 and PROX1 depend on BCL9 expression in colon cancer cells. Possibly, BCL9 might also play a role in the transcriptional regulation of these target genes in intestinal homeostasis.

In contrast, BCL9-2 expression was restricted to the mature differentiated epithelial cells, which reside in the villi. In the villi TGFß and BMP-signaling inhibit Wnt/ß-catenin signaling and function as growth repressors in differentiated epithelial cells (Haramis et al., 2004;Hardwick et al., 2004;Sancho et al., 2004). This inhibition through paracrine factors like BMPs is necessary for the differentiation of the cells into mature specialized cells (Crosnier et al., 2006; Hartenstein et al., 2010; He et al., 2004b). Interestingly, our studies in colon cancer cell lines showed that BMP4 gene expression is regulated by BCL9-2. Probably, BCL9-2 contributes to the expression of $\mathrm{Wnt} / \mathrm{B}$-catenin signaling repressive genes. Expression of the paracrine factor BMP4 could therefore be activated in intestinal epithelial cells. BMP4 leads to activation of BMP signaling in the mesenchyme that consequently inhibits Wnt signaling in the villi (Haramis et al., 2004; He et al., 2004b). BCL9-2 is not expressed in the crypts, where Wnt/ßcatenin signaling is active. Thus, BCL9-2 appears to be dispensable for intestinal Wnt/ß-catenin signaling which is restricted to the proliferative crypt compartment, although it might function in a ß-catenin-independent mechanism in the mature epithelial cells residing in the villi.

In accordance to our findings, Deka et al. showed that conditional ablation of both BCL9 proteins in intestinal epithelia did not disturb the normal intestinal homeostasis (Deka et al., 2010). The distribution and differentiation of intestine-specific cell lineages remained unaltered, although the expression of the stem-cell marker Leucine-rich repeat containing $G$ protein-coupled Receptor 5 (LGR5) and other intestinal epithelial genes, e.g. SOX6, were 
significantly reduced in intestinal epithelia of BCL9/BCL9-2 deficient animals (Deka et al., 2010). However, these studies do not provide detailed information about the particular function of BCL9 or BCL9-2, since the changes in the gene expression pattern of conditional $\mathrm{BCL} 9^{-/} / \mathrm{BCL} 9-2^{-1-}$ knockout mice may have been caused by both or just one of the proteins.

In our studies on colon cancer cells LGR5 mRNA expression was only dependent on ß-catenin and BCL9, while SOX6 expression was regulated by BCL9-2, suggesting diverse functions of BCL9 and BCL9-2 in malignant intestinal cells. Other intestinal canonical Wnt target genes such as SOX9 and PROX1 (Blache et al., 2004;Karalay et al., 2011;Petrova et al., 2002;Petrova et al., 2008) are dependent on ß-catenin and the BCL9 proteins. We found other differentially expressed genes in SW480 colon cancer cells after knockdown of ß-catenin and BCL9/BCL9-2. These genes are known to be important for intestinal homeostasis: the EPHB receptors and ephrin ligands were only partially dependent on ß-catenin in colon cancers (this work). Other studies have shown that EPHB receptors as Wnt/B-catenin target genes are key-regulators of migration and proliferation in the intestinal stem-cell niche (Holmberg et al., 2006). Further intestinal proteins like LGR5 (van der Flier et al., 2007) and SOX9 (Blache et al., 2004) have been shown to be expressed in the crypts in dependency of $\beta$-catenin , while BCL9-2 regulated proteins reside in the villi, e.g. CDX2 (Guo et al., 2004).

In summary, since previous investigations showed that knockout of BCL9 and BCL9-2 causes no obvious intestinal phenotype (Deka et al., 2010), it is conceivable that they are dispensable for normal intestinal homeostasis. However, the gene expression pattern of $\mathrm{BCL} 9^{-/ /} / \mathrm{BCL} 9^{-/-}$mutant epithelia was changed indicating that BCL9 and BCL9-2 regulate the expression of intestine specific genes whose functions are particular important for tumorigenesis (see below)(Brembeck et al., 2011;Deka et al., 2010). BCL9-2 might therefore participate in the regulation of the ephrinB ligands and CDX2 in a ß-catenin independent manner outside of the crypts. Additionally, canonical Wnt-signaling exerts a role in normal crypts by activating the expression of the EPHB receptors, CDX1, SOX9 and LGR5 probably with or without the contribution of BCL9 (Figure 37)(Batlle et al., 2002). 


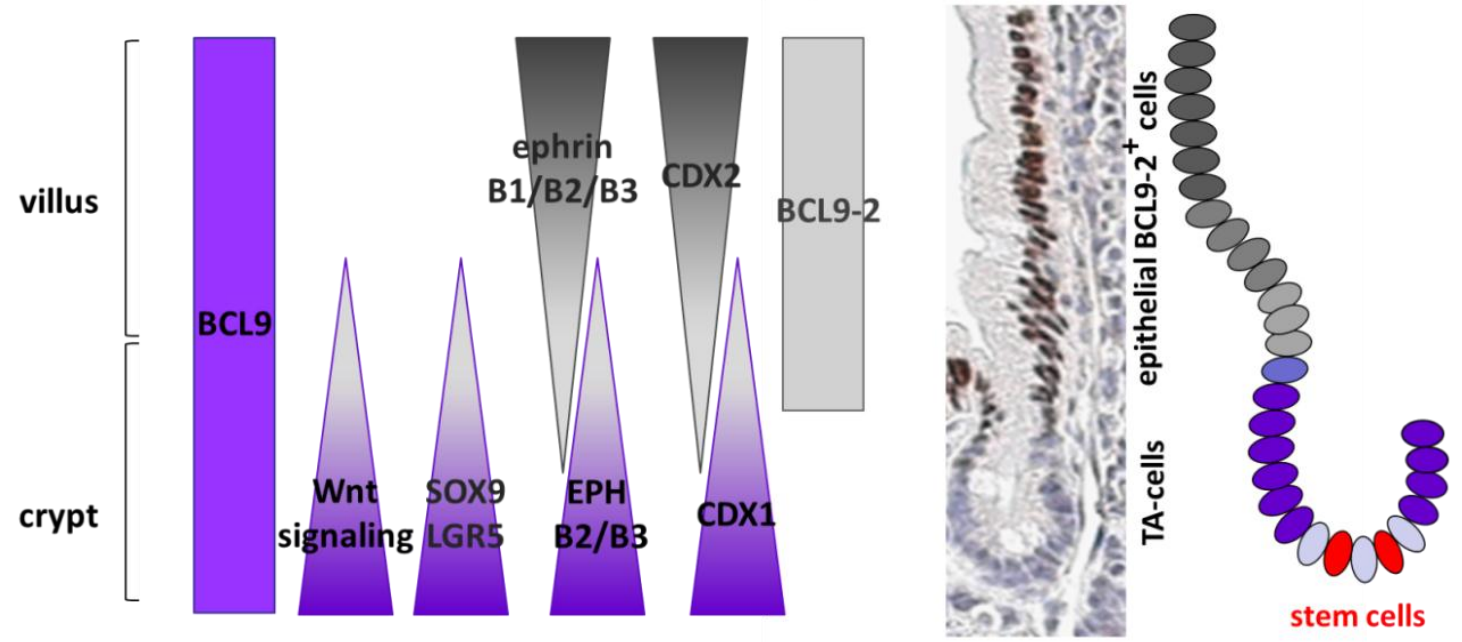

Figure 37: BCL9-2 expression and Wnt/ß-catenin signaling in the intestine

BCL9-2 is expressed in the villi of the intestine, apart from active Wnt/ß-catenin signaling in the crypts. Therefore BCL9-2 might contribute to the intestine specific gene expression pattern along the cryptvillus axis independently of ß-catenin. BCL9, in contrast, is expressed in all intestinal cells and has the spatial ability to contribute to ß-catenin-dependent gene transcription in the crypts.

\subsection{BCL9-2 is up-regulated independently of Wnt/ß-catenin signaling in early stages of intestinal tumorigenesis}

Previous analyses mainly focused on the overexpression of BCL9-2 in advanced tumors (Adachi et al., 2004;Sakamoto et al., 2007;Toya et al., 2007) while comprehensive analyses regarding early stages of tumorigenesis were still lacking. We found that early stages of tumor development, represented by adenomas of $\mathrm{APC}^{\mathrm{Min} /+}$ mice, exhibited high nuclear BCL9-2 protein expression. In contrast, BCL9-2 protein was absent in adjacent crypts. As already shown in mice, human tissue microarrays revealed significantly increased nuclear BCL9-2 protein in human adenomas whereas BCL9 protein expression remained unchanged compared to normal mucosa. Moreover, $90 \%$ of human colon cancers contained elevated or high BCL9-2 protein while high and elevated nuclear ß-catenin was present in only $59 \%$ of the colon cancer samples. In addition, we found that colon cancer cell lines carrying APC-mutations (Rowan et al., 2000) contain high levels of BCL9-2. In contrast, most cell lines with a ß-catenin stabilizing mutation (Kim et al., 2003) exhibited low or absent BCL9-2 protein expression. As already observed in $\mathrm{APC}^{\mathrm{Min} /+}$ tumors, BCL9 was ubiquitously expressed in colon cancer cell lines at approximately equal levels compared to normal mucosa. Here, we show that BCL9-2 protein expression is significantly increased in early stages of tumorigenesis and highly expressed in tumors harboring an APC mutation while BCL9 expression is not altered. 
Previous studies provide evidences for a dependency of BCL9-2 expression on Wnt/ß-catenin signaling (de la Roche et al., 2008). De la Roche et al. found increased BCL9 and BCL9-2 mRNA expression following Wnt3a stimulation of HEK293 cells. However, we did not observe any reduction of BCL9/BCL9-2 mRNA or protein levels after knockdown of ß-catenin in SW480 colon cancer cells indicating that the expression of BCL9 and BCL9-2 in colon tumorigenesis does not depend on Wnt/ß-catenin signaling. Moreover, BCL9-2 mRNA and protein expression was even significantly induced in SW480 cells after knockdown of ß-catenin. These data suggest that BCL9 and BCL9-2 are not targets of ß-catenin and that Wnt/ß-catenin signaling might negatively regulate the expression of BCL9-2. It has been shown that LEF1 is activated through binding of ß-catenin that consequently results in suppression of E-cadherin gene transcription in keratinocytes (Jamora et al., 2003). Possibly ß-catenin negatively regulates BCL9-2 expression in colon cancer cells through a similar mechanism. However, the precise undelaying mechanism remains to be discovered.

Further findings in vivo support our hypothesis that ß-catenin negatively regulates BCL9-2 expression. The crypt compartment in normal intestine lacks BCL9-2, which is probably due to the negative influence of B-catenin on BCL9-2 transcription. In the villi, ß-catenin resides at the cell membrane and therefore exerts no transcriptional function. Thus, BCL9-2 expression is restricted to the villi.

The negative effect of ß-catenin on BCL9-2 expression might be overcome by other mechanisms, which lead to overexpression of BCL9-2. For instance, we found elevated levels of BCL9-2 protein in adenomas of $\mathrm{APC}^{\mathrm{Min} /+}$ mice (this study). Loss of function mutations in APC are associated with chromosomal instability (CIN) (Alberici and Fodde, 2006;Caldwell and Kaplan, 2009;Fodde et al., 2001). CIN is a common event in tumorigenesis often resulting in the translocation of genetic loci e.g. of oncogenes (Caldwell et al., 2009;Dikovskaya et al., 2007; Radulescu et al., 2010). Also, BCL9 overexpression was linked to trans-location of its gene locus in B cell malignancies (Willis et al., 1998). It is possible that the gene locus of BCL9-2 could be also rearranged likewise resulting in increased transcription levels. Indeed, the chr11q22 which harbors the gene locus of BCL9-2, was found to be altered in $64 \%$ of colon cancers (Knosel et al., 2002). Accordingly, ilt might be possible that gene re-arrangement of the BCL9-2 gene locus occur due to chromosomal instability induced by APC mutations. 


\subsection{BCL9-2 promotes intestinal tumorigenesis}

\subsubsection{BCL9-2 expression enhances Wnt/ß-catenin signaling activity in intestinal tumorigenesis}

The majority of colon cancers show high levels of canonical Wnt-signaling due to mutations causing ß-catenin stabilization or APC truncation, since these mutations lead to aberrant transcriptional activity of ß-catenin (Nagase et al., 1993;Polakis, 2000;Reya and Clevers, 2005; van der Flier et al., 2007). We analyzed the level of Wnt/ß-catenin signaling in different colon cancer cell lines that contain high, moderate or low levels of BCL9-2 and found that BCL9-2 protein levels correlated with the level of Wnt/ß-catenin signaling activity in these cell lines.

The dosage of Wnt/ß-catenin signaling is crucial for many different events, including normal developmental processes, such as the maintenance of pluripotency and intestinal specification (Gadue et al., 2006;Kemp et al., 2005;Mohamed et al., 2004;Nostro et al., 2008;Sherwood et al., 2011). In addition, adult tissues require a well-defined dosage of canonical Wnt-signaling: intestinal homeostasis (Pinto et al., 2003) and pathological events like the formation of intestinal tumors and breast cancers (Mohinta et al., 2007; Reya et al., 2005).

Since the level of Wnt/ß-catenin activity is critical for cell renewal and differentiation, many regulatory mechanisms exist which control the Wnt/ß-catenin-dependent activation of target genes. In embryonic development and homeostasis paracrine signaling molecules form concentration gradients. These lead to different signaling responses which subsequently define the resulting tissues. For example, in the adult intestine APC is inversely expressed to active ßcatenin along the crypt villus which results in a gradient of canonical Wnt-signaling activity (Gaspar and Fodde, 2004). In addition, nuclear repressors and activators modulate the function of ß-catenin: Groucho generally represses ß-catenin dependent transcription through the recruitment of repressive chromatin (Fisher and Caudy, 1998;Palaparti et al., 1997), while other transcriptional co-regulators like CBP/p300 can either activate or repress canonical Wnt signaling in dependency of the recruited co-factors (Li et al., 2007).

Additionally, BCL9-2 modulates the activity of canonical Wnt-signaling in different ways.

Our group previously showed that BCL9-2 promotes the trans-location of $\beta$-catenin to the nucleus that enhances Wnt/ß-catenin-dependent target gene transcription (Brembeck et al., 
2006;Brembeck et al., 2004). In addition, BCL9-2 modulates the switch between ß-catenin's transcriptional and adhesive function due to the preferred binding of Y142 phosphorylated Bcatenin to BCL9-2 instead of $\alpha$-catenin (Brembeck et al., 2004). Moreover, several studies showed that BCL9 and BCL9-2 co-activate the transcription of ß-catenin target genes (Adachi et al., 2004;Brembeck et al., 2004;Brembeck et al., 2011;Deka et al., 2010;Sustmann et al., 2008).

Because BCL9-2 appears to be redundant for normal intestinal homeostasis but exerts tumorigenic properties, some studies aimed to target the interaction between ß-catenin and BCL9/BCL9-2 to inhibit tumor growth. Takada and colleagues developed a stabilized $\alpha$ helix of BCL9 (SAH-BCL9), which interferes with its ß-catenin binding domain. This resultes in an impaired binding to ß-catenin. Remarkably, SAH-BCL9 led to the suppression of tumor growth, angiogenesis, invasion and metastasis formation in mouse xenografts. In addition, de la Roche et al. identified carnosic acid to inhibit $\beta$-catenin binding to the BCL9 co-factors (de la Roche et al., 2012b). Their study demonstrated that binding to BCL9 proteins protect unphosphorylated $\beta$-catenin against degradation. Thus, treatment of colon cancer cells with carnosic acid lead to destabilization of oncogenic $\beta$-catenin and consequently to transcriptional inactive oncogenic ß-catenin (de la Roche et al., 2012b).

In summary, the function of BCL9-2 in canonical Wnt-signaling is most likely the enhancement of a critical signaling threshold which results in the activation of certain Wnt target genes in pathologic processes. The $\mathrm{Wnt} / \mathrm{\beta}$-catenin signaling pathway plays a crucial role for the balance of stemness and differentiation in the intestinal crypts (Fodde and Brabletz, 2007). However, Fodde and colleagues resumed that ß-catenin stabilizing APC mutations alone might not be sufficient to cause aberrant $\beta$-catenin transcriptional activation. Additional factors appear to be necessary to induce a pathological transcription level, which leads to tumorigenesis (Gaspar et al., 2004), for instance, BCL9-2, which enhances the ß-catenin signaling output. Our studies support this role, since Wnt/ß-catenin activity correlates with the protein level of BCL9-2 in colon cancer cell lines. Overexpression of BCL9-2 results in the transcriptional co-activation of Wnt/ß-catenin target genes. This might be promoted by BCL9-2 mediated nuclear retention of B-catenin and the regulation of ß-catenin's transcriptional function. Although BCL9/BCL9-2 appear to be dispensable for intestinal homeostasis, they play an important role in pathologic processes. 


\subsubsection{BCL9-2 is not required for the expression of all canonical Wnt target genes and regulates additional ß-catenin-independent genes implicated in tumorigenesis}

BCL9-2 and BCL9 are potent co-activators of canonical Wnt-signaling. We addressed the question whether the proteins are essential or just partially required co-factors for $\mathrm{Wnt} / \mathrm{B}$ catenin signaling or whether they regulate target genes independently of ß-catenin. We analyzed different Wnt/ß-catenin-dependent Luciferase-reporters and the endogenous expression of known ß-catenin-target genes and other genes implicated in colon cancer. To this end, the expression of the target genes was analyzed after knockdown of BCL9 and BCL9-2 in comparison to the knockdown of ß-catenin in colon cancer cells.

Knockdown of BCL9-2 and BCL9 resulted in strong reduction of Axin2 and TOP/FOP Wnt reporter activities in cell lines with high levels of BCL9-2. In addition, we found several endogenous Wnt target genes which were highly dependent on BCL9-2 and BCL9, e.g. Bambi, TCF1 and EPHB2. But not all ß-catenin target genes were regulated by the BCL9 proteins. The CyclinD1 reporter was not affected by BCL9 or BCL9-2 knock down. In line with these findings, CyclinD1 mRNA was not changed after knock down of the BCL9 proteins. In addition, genes such as LEF1 and MSX2 also not responded to BCL9 and BCL9-2. Thus, BCL9-2 promotes only a subset of Wnt/ß-catenin target genes (Figure 38).

Interestingly, Clarke and Clevers recently postulated that CyclinD1 is not a direct target gene of ß-catenin. They found that endogenous CyclinD1 expression was not altered following antagonism of the Wnt pathway in vitro or even was not induced by conditional loss of APC in vivo (Sansom et al., 2005). In consequence it is possible that also BCL9-2, as co-factor of canonical Wnt-signaling, is not able to induce CyclinD1 expression. Thus, secondary effects appear to be required for CyclinD1 overexpression in cancers. For example, p53 mutations lead to activation of NFKB, which positively regulated CyclinD1 transcription (Rocha et al., 2003). Therefore, it is likely that ß-catenin regulates the expression of CyclinD1 by a BCL9-2independent activation of an inhibitor or by inhibition of a suppressor such as p53. However, the underlying mechanism is still unknown and needs to be investigated.

BCL9-2 independent ß-catenin target genes are co-regulated by other factors and obviously do not require the co-activation through the BCL9 proteins. For example, MSX2 was shown to be co-regulated by cooperative binding of SMAD4 and LEF1 in murine embryonic stem cells (Hussein et al., 2003). Interestingly, Hussein and colleagues found, that Wnt/ß-catenindependent activation of MSX2 required the presence and functionality of SMAD binding 
elements. Moreover, we found that the mRNA expression of Bambi was highly dependent on BCL9-2 in colon cancer cells. Expression levels of Bambi, an inhibitor of TGFß-signaling, were shown to correlate with the metastasis-free survival time of colon cancer patients. In addition, Fritzmann et al. found that overexpression of Bambi increases migration of colon cancer cells and subsequently metastasis formation (Fritzmann et al., 2009).

BCL9-2 and BCL9 are not required for all ß-catenin target genes, as our studies demonstrated. Microarray analyses and qPCR of different cancer cell lines supported these observations. Each of the BCL9 proteins mediated its own set of ß-catenin- target genes (Figure 38). Thus, genes like LGR5 were dependent on B-catenin and BCL9 but not on BCL9-2 levels. In addition, CDX1 and BMP4 were co-regulated by $\beta$-catenin and BCL9-2, and independent of BCL9 in colon cancer cells.

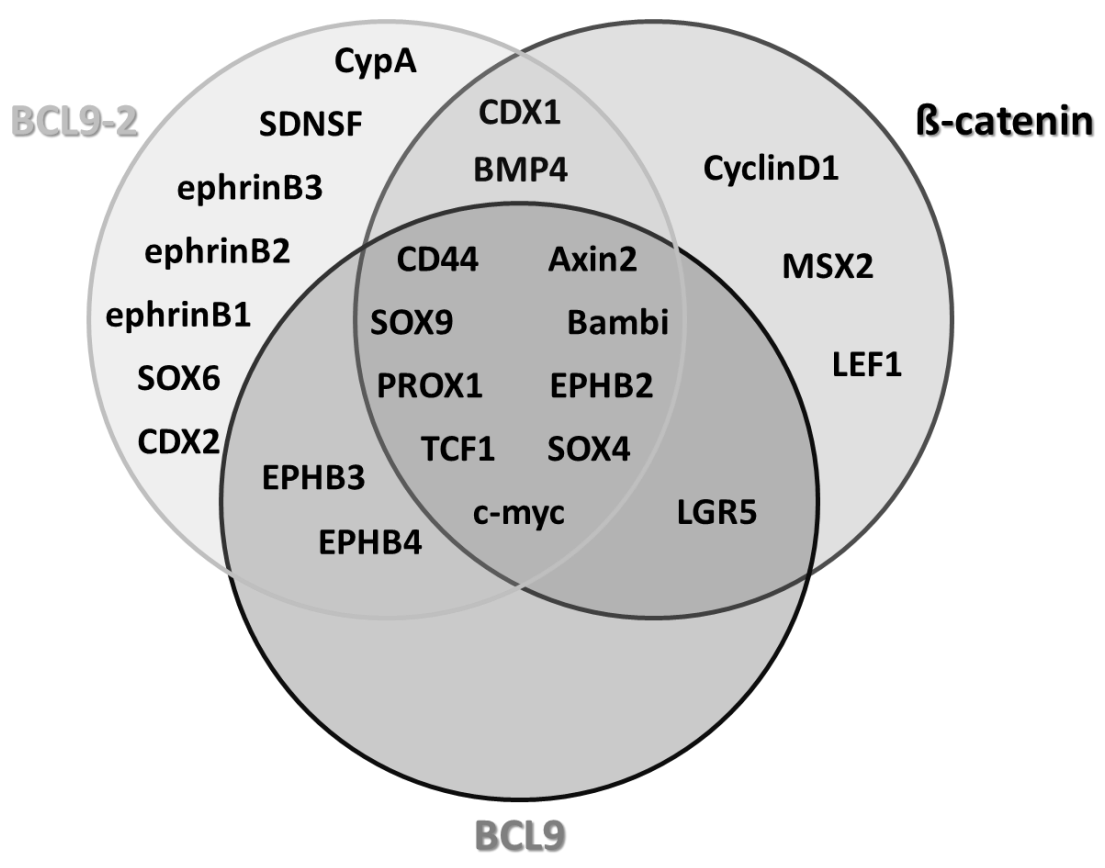

Figure 38: BCL9-2 and BCL9 regulate a subset of $\mathrm{Wnt} / \mathrm{B}$-catenin target genes and regulate the expression of distinct genes independently of ß-catenin

Venn diagram showing the different genes which were identified in this study to be transcriptionally regulated by BCL9, BCL9-2 or ß-catenin, as indicated.

Interestingly, BCL9-2 induced an additional set of target genes which was apparently independent of ß-catenin indicating that BCL9-2 controls the expression of ß-catenin independent genes in colon and breast cancer. This gene set includes CDX2, the EPHB3/4 receptors and ephinB1/2/3 ligands (Figure 38). 
These BCL9-2 target genes might further trigger intestinal tumorigenesis. As we have shown for BCL9-2, the ephrinB1, B2 and B3 ligands and EPHB3 and B4 (Hafner et al., 2004;Lugli et al., 2005) receptors, as well as CDX1 and CDX2 (Ee et al., 1995;Ren et al., 2000;Silberg et al., 1997) have been described to be up-regulated in early phases of intestinal tumorigenesis.

Investigations regarding the function and expression of CDX1 and CDX2 are partially controversial. Both proteins have been described to exert tumor-promoting and -repressing functions. However, CDX1 and CDX2 are up-regulated in intestinal adenomas (Ee et al., 1995;Ren et al., 2000;Silberg et al., 1997) and to some extent silenced in carcinomas. For example, CDX2 expression might be maintained in most colon cancers and lost in tumors with a high frequency of microsatellite instability (Hinoi et al., 2001).

The underlying mechanisms which lead to the repression of the proteins are not completely understood yet. It has been suggested that hypermethylation of the CDX1 promoter causes transcriptional silencing (Suh et al., 2002). In addition, CDX2 silencing was shown to be in part mediated by a dominant negative transcriptional repressor (Hinoi et al., 2003). CDX1 was hypothesized to promote proliferation in different colon cancer cell lines (Di Guglielmo et al., 2001; Soubeyran et al., 2001), whereas other studies claimed that CDX1 inhibits Wnt/ß-catenin signaling and thereby exerts anti-proliferative functions (Lynch et al., 2003).

However, our results show that CDX1 and CDX2 are expressed in different colon cancer cell lines in dependency of BCL9-2 expression. Some studies determined a tumor-suppressive function of CDX2 (Lorentz et al., 1997; Mallo et al., 1998). In contrast, others claimed that CDX2 exerts tumor promoting functions (Bonhomme et al., 2003;Oshima et al., 1995), which to some extent reflect the influences we observed after BCL9-2 overexpression in our transgenic mouse model. For example, Salari et al. demonstrated that CDX2 is de-regulated in colorectal cancers through chromosomal focal amplification and acts as a lineage-survival oncogene. Thus, cancer cells deriving from the CDX2-lineage require the presence of this oncogene for continued growth and survival. Moreover, the study of Salaris et al. claimed that CDX2 is implicated in Wnt/ $\beta$-catenin signaling and therefore contributes to colorectal tumorigenesis (Salari et al., 2012). In addition, it was recently shown that CDX2 regulates claudin1 expression (Bhat et al., 2012), which has been linked to tumor progression and metastasis in association with EMT (Dhawan et al., 2005). Thus, BCL9-2 dependent activation of CDX2 might further promote the epithelial-mesenchymal transition of cancer cells.

In conclusion, the function of CDX1 and CDX2 depends on their temporally and locally expression. It is likely that, depending on the genetic background, the proteins exert different 
functions. Further investigation will provide more insights into the complex role of the CDX proteins by dissecting the tumor-promoting and -repressing functions in dependency on the cellular context.

In addition to CDX proteins, the expression of the EPHB receptors and ephrinB ligands were strongly dependent on BCL9-2. In intestinal homeostasis, the EPHB/ephrins have been shown to regulate cell migration and intestinal cell fates through contact-mediated cell repulsion (Sancho et al., 2003;Sancho et al., 2004). This function suggests a role of the proteins for tumor invasion. Many studies have shown that the EPHs and ephrins are overexpressed in early stages of tumorigenesis and silenced in advanced stages due to hypermethylations of their promoters (Hafner et al., 2004;Lugli et al., 2005). Although overexpression of the Wnt/ßcatenin target EPHB2 is mainly associated with loss of cancer progression, further studies provided evidence that aberrant expression of the ß-catenin independent BCL9-2 target gene products EPHB4 and ephrinB2 give rise to metastases (Liu et al., 2002b;Liu et al., 2004; Stephenson et al., 2001). However, up-regulation in particular of the BCL9-2 regulated $\mathrm{EPHB} / \mathrm{ephrins}$ was shown to promote tumor invasion which was also observed as a consequence of BCL9-2 overexpression.

Moreover, using microarray analyses we identified the new BCL9-2 core target genes Cyclophilin A (СурА) and the stem-cell-derived neural stem/progenitor cell supporting factor (SDNSF) which were independent of ß-catenin and BCL9 in cancer cell lines (Figure 38). SDNSF and СурА are novel candidates which could further trigger tumor progression by overexpression of BCL9-2. Both proteins had been previously shown to be implicated in tumorigenesis (Gashaw et al., 2007;Mosca et al., 2010;Obchoei et al., 2009).

The physiological function of SDNSF is the transport of selected proteins as component of a receptor which is important for the endoplasmatic reticulum (ER) - Golgi apparatus (Zhang et al., 2003). SDNSF supports the survival and multipotency of neuronal stem/progenitor cells without the addition of fibroblast growth factor (FGF) or epidermal growth factor (EGF) in vitro, which are indispensable for retention of the potential to self-renew under physiological conditions (Toda et al., 2003). The EGF-signaling pathway was shown to regulate colon cancer stem-cell proliferation and apoptosis (Feng et al., 2012). Possibly, SDNSF promotes the maintenance of pluripotency of cancer cells, which is a common event in tumorigenesis (Abdul Khalek et al., 2010). In this regard SDNSF might contribute to the autonomy and independence of tumor cells on self-renewing promoting factors like FGF or EGF. However, detailed studies regarding the expression and role of SDNSF in tumorigenesis are still missing: Gashaw and 
colleagues postulated SDNSF to be marker for testicular germ cell tumors (Gashaw et al., 2007). Moreover, some studies provided evidences that SDNSF is up-regulated in breast cancers (Mosca et al., 2010).

Cyclophilin A (СуpA), the second newly identified BCL9-2 target gene is a peptidylprolyl cistrans-isomerase (PPI) which accelerates the folding of proteins and is involved in intracellular protein trafficking (Andreeva et al., 1999. CypA is overexpressed in many tumor, e.g. in pancreas, breast and colon cancer (Obchoei et al., 2009). Importantly, overexpression of CypA prevents hypoxia- and cisplatin-induced apoptosis in HCT116 colon cancer cells but has no influence on cell proliferation (Choi et al., 2007). In addition, it has been shown that the new BCL9-2 target gene is a promising candidate for treatment and early diagnosis of diverse cancers like hepatocyte and endometrial tumors (Lee, 2010a;Lee, 2010b;Obchoei et al., 2009).

However, detailed analyzes for the role of SDNSF and CypA in intestinal tumors are missing. Further investigations regarding their function and expression during intestinal tumorigenesis could further shed light on the role of BCL9-2 in colon cancer.

In summary, this study revealed that BCL9-2 plays a more prominent role than BCL9 for the coactivation of tumorigenesis-related genes. Interestingly, both co-factors mediate a gene signature which is apparently independent of ß-catenin in cancer cells. In addition, we identified CypA and SDNSF as new BCL9-2 target genes. SDNSF and CypA were both shown to be overexpressed and to some extent implicated in the maintenance of tumor-related properties. This supports our hypothesis that target gene products of BCL9-2 trigger the $\beta$ catenin independent, oncogenic role of this protein. 


\subsubsection{BCL9-2 promotes tumor development and local invasion}

BCL9-2 is up-regulated in the majority of colon cancers. It has been shown, that the phenotypes of aberrant expressed BCL9-2 target genes result in severe phenotypes (see above and the following text). We used a BCL9-2 transgenic mouse model, to investigate whether BCL9-2 overexpression is involved in tumor development and progression in vivo. In our model, BCL9-2 overexpression in mice was achieved by a keratin 19 (K19) promoter which leads to expression of the transgene in simple epithelia including the intestine (Brembeck et al., 2011).

BCL9-2 overexpression alone induced the development of undifferentiated tumors in the small intestine in aged mice (>15 month) with a relatively low incidence of $20 \%$. To analyze whether BCL9-2 overexpression contributes to tumor formation in combination with other genetic alterations in vivo, adenoma development was analyzed in compound $\mathrm{APC}^{\mathrm{Min} /+} ; \mathrm{K} 19-\mathrm{BCL} 9-2$ mice.

Remarkably, overexpression of $\mathrm{BCL9-2}$ in compound $\mathrm{APC} \mathrm{Min} /+^{+} ; \mathrm{K} 19-\mathrm{BCL9}-2$ mice resulted in significantly increased adenoma formation in the small intestine with regard to number and size compared to $\mathrm{APC}^{\mathrm{Min} /+}$ non-transgenic littermates. The same tendency was observed in the colon, although the changes were not significant due to the rare development of colonic tumors in $\mathrm{APC}^{\mathrm{Min} /+}$ mice per se. The life span of APCMin/+ mice is generally shortened due to anemia resulting from the intestinal adenomas (Moser et al., 1990).

Our results are in agreement with studies by Deka et al. for intestinal tumorigenesis in BCL9/BCL9-2 mutant animals: Induction of dysplastic adenomas by dimethylhydrazine in BCL9/BCL9-2 knockout mice led to tumor formation with similar incidences in knockout and wild type mice. However, the size of $\mathrm{BCL} 9^{-1-} / \mathrm{BCL} 9-2^{-1-}$ derived tumors was significantly decreased in comparison to wild type mice. Thus, loss of BCL9/BCL9-2 suppresses tumor growth. Accordingly, APC ${ }^{\mathrm{Min} /+} ; \mathrm{K} 19-\mathrm{BCL} 9-2$ animals developed huge adenomas, indicating that BCL9-2 overexpression leads to tumor development under supra-pathological conditions. APC mutation resulting in ß-catenin stabilization alone were suggested to be not sufficient to cause aberrant ß-catenin transcriptional activation (Gaspar et al., 2004). Thus, our studies reveal that BCL9-2 overexpression induces an increased supra-pathological transcription level of ßcatenin-dependent and -independent target genes, which further promote tumor progression.

Remarkably, adenomas of $\mathrm{APC}^{\mathrm{Min} /+} ; \mathrm{K} 19-\mathrm{BCL9}-2$ mice were locally invasive with tumor cells growing invading the submucosa and muscularis, which was never observed in APC ${ }^{\mathrm{Min} /+}$ control 
mice. APC ${ }^{\mathrm{Min} /+}$ mice develop benign tumors with well-formed boundaries (Moser et al., 1990). Thus, canonical Wnt-signaling requires the co-activation of genes by additional factors to induce invasion, as observed in our compound mutant mice (see 5.3.1).

This study identified the Wnt/ß-catenin target gene PROX1 to be highly dependent on BCL9-2. Indeed, it has been shown that ß-catenin alone is not sufficient to activate PROX1 gene expression in colon cancer cells (Petrova et al., 2008). Petrova and colleagues demonstrated that PROX1 is a dose-dependent target gene of Wnt/ß-catenin signaling. Moreover, a yet unknown factor is required for transcriptional activation of PROX1 in addition to nuclear $\beta$ catenin. Our study suggests that BCL9-2 might be the missing link to induce the specific activation of target genes like PROX1. Accordingly, high PROX1 expression was found in SW480 and HT29 (Petrova et al., 2008) which contain high levels of BCL9-2 (this study). In contrast, DLD1, WiDr and HCT-116 cells with no or moderate BCL9-2 protein expression (this study) were negative for PROX1 (Petrova et al., 2008). According to BCL9-2, overexpression of PROX1 promotes intestinal tumor progression and invasion (Petrova et al., 2008). Overexpression of PROX1 correlates with poor prognosis in colon cancer patients (Skog et al., 2011). Elyada et al. provided evidence, that PROX1 mediates invasion through the alteration of cell-polarity and adhesion in a TP53-dependent manner (Elyada et al., 2011). However, the detailed underlying mechanism has not been described so far. Future experiments are required to investigate the dependency of PROX1 on BCL9-2 in more detail.

We already showed, that invasive areas of $\mathrm{APC}^{\mathrm{Min} /+} ; \mathrm{K} 19-\mathrm{BCL9}-2$ mice derived tumors express the 3 -catenin/BCL9-2 regulated EPHB2 and Bambi as well as the BCL9-2 dependent EPHB3 and B4 proteins, which were differentially regulated by ß-catenin. The expression of the ephrinB3 and EPHBB4 receptors was negatively regulated by ß-catenin in SW480 suggesting that ßcatenin overexpression might cause inhibition of these genes in colon tumors. Moreover, deregulation of EPHB4 and ephrinB2 was shown to correlate with metastases formation (Liu et al., 2002b; Liu et al., 2004;Stephenson et al., 2001). This findings support the hypothesis that BCL9-2 is capable of regulating the transcription of target genes independently from ß-catenin which promote tumorigenesis. In addition, BCL9-2 might be essential for the expression of $\beta$ catenin target genes like PROX1, which further contribute to invasion.

Invasion is a process which requires the activity of proteins that contribute to the disruption of the extracellular matrix and proteins which induce a motile, epithelial phenotype of the cancer cells (reviewed in (Kong et al., 2011;Yilmaz and Christofori, 2009). Our studies provide evidences that BCL9-2 overexpression contributes to the malignant phenotype of cancer cells 
through the promotion of epithelial-mesenchymal transition (EMT). Accordingly, we observed a transition of the mesenchymal-like phenotype of cancer cells to a more epithelial-like morphology after knock down of BCL9-2 and to a lesser extend after BCL9 knockdown. Earlier studies from our group showed that BCL9-2 negatively regulates the mRNA expression of the epithelial marker E-cadherin, which supports our hypothesis that BCL9-2 contributes to EMT. Also in zebra fish embryos, BCL9-2 was shown to trigger EMT (Brembeck et al., 2004). In agreement with our findings, Deka and colleagues found that $\mathrm{BCL} 9^{-1-} / \mathrm{BCL} 9-2^{-/-}$mutant tumors exhibited a reduced expression of target genes which had been associated with EMT (e.g., Branchury (T) and Vimentin (Vim)), intestinal stem-cell traits (e.g. LGR5) (Deka et al., 2010). Moreover, in addition to $\mathrm{Wnt} / \mathrm{B}$-catenin-dependent mesenchymal markers, further characteristic EMT-key player genes were reduced in the gene set of $\mathrm{BCL} 9^{-/} / \mathrm{BCL}-2^{-/}$mutant tumors, e.g. snail homolog 2 (SLUG), TWIST1 and zinc finger E-box binding homeobox 1 and 2 (ZEB1/2). Until now it is not clear whether BCL9-2 itself participates in the transcriptional regulation of EMT key components like SLUG and SNAIL, or whether only an indirect process underlies the promotion of EMT like the activation of PROX1 or the inhibition of E-cadherin expression. Therefor future experiments need to examine the influence of BCL9-2 on the regulation of EMT marker genes in cancer cells.

Taken together, our findings indicate that BCL9-2 overexpression promotes invasion through the contribution to epithelial-mesenchymal transition (EMT) directly or indirectly through the activation of EMT-promoting factors. In line with these results, Deka et al. claimed that BCL9/BCL9-2 expression is associated with LGR5-positive intestinal multipotent stem-cells and consequently with cancer stem-cells that exert stem-cell like properties (Deka et al., 2010).

In summary, BCL9-2 promotes intestinal tumorigenesis and local invasion. These features are obviously mediated by two different mechanisms: First, aberrant expression of BCL9-2 increases canonical Wnt signaling and many of the Wnt/B-catenin/BCL9-2 target gene products have been described by to mediate tumor promoting properties like invasiveness (Han et al., 2012). Second, BCL9-2 activates its own gene set and thereby triggers tumor development and invasion and that BCL9-2 further promotes epithelial-mesenchymal transitions. 


\subsection{A novel mechanism for BCL9-2 to regulate target gene transcription independently of ß-catenin}

Here we identify a mechanism which is responsible for the $ß$-catenin independent activation of BCL9-2 target genes. We used a system which allows the comparison of the transcriptional regulation of a ß-catenin/BCL9-2-dependent- (CDX1) and a ß-catenin independent BCL9-2 target gene (CDX2) in colon cancer cell lines. Similar to BCL9-2, the caudal related homeobox genes CDX1 and CDX2 are up-regulated in early stages of intestinal carcinogenesis (Ee et al., 1995; Ren et al., 2000;Silberg et al., 1997). We found that BCL9-2 regulates the expression of CDX1 and CDX2. Interestingly, only CDX1 was dependent on the expression of ß-catenin while CDX2 appeared to be negatively regulated.

\subsubsection{BCL9-2 regulates the expression of CDX1 and CDX2 independently of ß-catenin in colon cancer cells}

During mouse embryonic development, the initiation of CDX2 transcription is induced by canonical Wnt-signaling at around E8.0. However, CDX2 expression was suggested to be independent of B-catenin in later stages and adults (Sherwood et al., 2011). These observations are in line with our studies in colon cancer cells: ß-catenin knockdown had no influence on the CDX2 mRNA expression in colon cancer cells. Moreover, our studies suggest, that the LEF binding elements also known as Wnt responsive elements (WRE) in the proximal promoter of CDX2 are dispensable for BCL9-2 mediated reporter gene activity. This indicated that BCL9-2 acts independently of B-catenin in the regulation of these target genes.

Further studies showed that the $-3600 \mathrm{bp}$ proximal promoter of CDX1 harbors four Wnt responsive elements which are essential for embryonic development (Lickert et al., 2000). However, the Wnt responsive elements in the $-386 \mathrm{bp}$ promoter of CDX1 were shown to be not necessary for the activation of CDX1 expression in later stages than E8.5 (Lickert et al., 2000). Our study confirmed that the Wnt responsive elements in the CDX1 proximal promoter are not functional colon cancer cells. In contrast, endogenous CDX1 expression was dependent on $\beta$ catenin, obviously through more distal Wnt responsive elements. Lickert and colleagues showed that Wnt/ß-catenin signaling regulates CDX1 expression in intestinal tumors in mice (Lickert et al., 2000). Our results support this observation. 
In conclusion, only BCL9-2, but not ß-catenin, regulates the endogenous expression of CDX2 in colon cancer cells. Moreover, we found that BCL9-2 regulates the expression of CDX1 through the proximal promoter probably independent from ß-catenin.

However, since BCL9-2 mediates the transcriptional activation of diverse Wnt/B-catenin target genes we cannot exclude that BCL9-2 acts also on more distal Wnt responsive elements in the promoter of target genes. Here we focused on the proximal promoters of CDX1/2 and suggest a ß-catenin independent function of BCL9-2 to regulate the basal transcription of these genes. (Figure 40).

\subsubsection{Identification of a new OCT4 responsive, transcription factor binding element in the proximal promoters of CDX1 and CDX2}

Here, we identified OCT4 as a novel transcriptional activator of CDX1 and CDX2 gene expression. OCT4 and CDX2 are key transcription factors together with NANOG, SRY (sex determining region Y)-box 2 (SOX2) and gut-enriched Krüppel-like factor (KLF4) in early embryonic development where they define the lineage decisions in the mouse blastocyst (Niwa et al., 2000;Pesce and Scholer, 2001; Wei et al., 2009;Zhang et al., 2010). CDX2 expression leads to trophoblast lineage differentiation, whereas OCT4, NANOG, SOX2 and KLF4 expression are necessary for the self-renewing potential of pluripotent stem-cells (Niwa et al., 2000). In this context both, OCT4 and NANOG are necessary to inhibit CDX2 expression through binding to cis-regulatory elements in the CDX2 promoter (Chen et al., 2009). CDX1 is expressed in later stages during embryonic development and not correlated with OCT4 expression (Lickert et al., 2002).

None of the already described OCT4-binding elements are present in the proximal promoter constructs used in our studies. Surprisingly, mutation of a newly identified OCT4 binding element in the proximal promoter of $\mathrm{CDX} 2$ led to strong reduction of reporter activity. Moreover, OCT4 overexpression activated CDX1 and CDX2 reporter activities through the novel OCT4 binding element in each of the promoters. This indicates that OCT4 is an essential transcription factor for CDX2 reporter activity. Thus, our analyses revealed that OCT4 exerts distinct functions in intestinal tumorigenesis and embryonic development.

We found that BCL9-2 driven CDX2 reporter activity requires the functionality of the novel OCT4 binding element in colon cancer cell lines. Further analyses are required to analyze the putative interaction of OCT4 and BCL9-2 (Figure 39A). Our preliminary studies showed that 
overexpressed BCL9-2 did not bind to exogenous expressed OCT4 in HEK293 cells, suggesting an indirect binding of BCL9-2 to OCT4. In addition, BCL9-2 might modulate the expression of CDX2 by binding to the OCT4 responsive element through another DNA-binding protein such as OCT1 (Figure 39A) or OCT4 mediated transcription requires a co-factor which is dependent on BCL9-2 expression (Figure 39B). Thus, we will further investigate the BCL9-2 dependent function of OCT4 in colon cancer cell lines. Consequently, the expression of other OCT4 known target genes, e.g. NANOG and SOX2 (Jung et al., 2010) will be analyzed. Furthermore, BCL9-2 might also modulate the expression of OCT4 itself or of another related transcription factor (Figure 39B), which mediates the transcriptional activation of CDX2 through the novel OCT4binding element.

\section{$----\rightarrow$ BCL9-2 mediated transcriptional regulation \\ $\longrightarrow$ binding of transcription factors to DNA-binding elements}
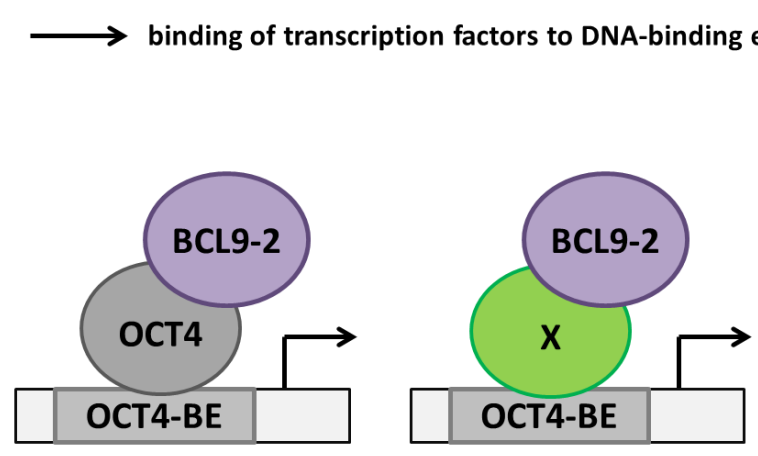

A.

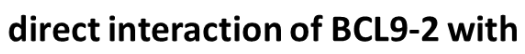

$$
\text { OCT4-BE bound factor }
$$
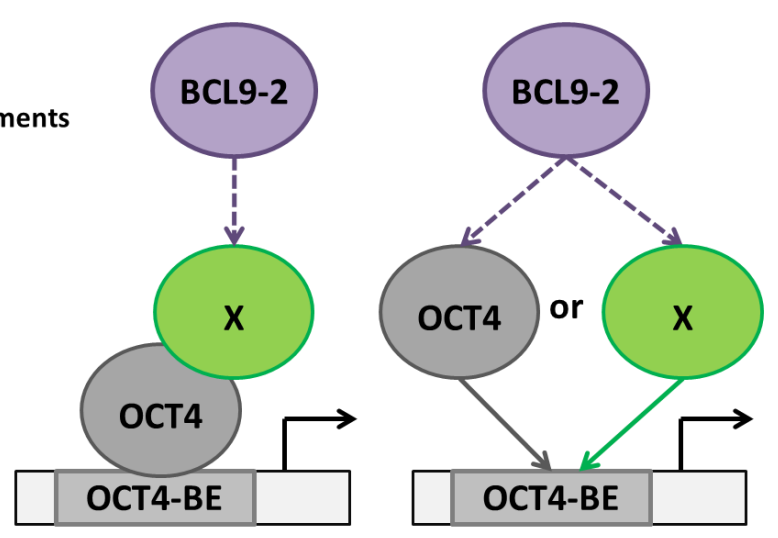

B.

\section{primary/secondary regulation of OCT-BE} bound factor by BCL9-2

Figure 39: Overview about putative mechanisms for BCL9-2/OCT4 transcriptional activation of CDX2. BCL9-2 might either act through (A) direct interaction with OCT4 or an related transcription factor or (B) secondary, through the transcriptional regulation of OCT4, of an OCT4-required co-factor, or of a transcription factor, which binds to the OCT4-binding element (OCT4-BE).

OCT4 is only expressed in embryonic stem-cells and primary silenced in adults with rare exceptions of some adult stem-cells such as breast- and skin-stem-cells (Pesce et al., 2001;Tai et al., 2005). However, the function of OCT4 in cancer cells appears to be different to its function in embryonic stem cells (this study). Overexpression of CDX2 in mESCs induces trophoblast differentiation (Tolkunova et al., 2006), while knockdown of OCT4 results in endoderm and trophoblast differentiation (Hay et al., 2004). Interestingly, several publications reported that OCT4 is expressed in different breast cancer and colon cancer cell lines (Jin et al., 1999;Steingart et al., 2002; Wang et al., 2003). Overexpression of OCT4 induces the reactivation of pluripotency associated factors which contribute to tumorigenesis (Hochedlinger et al., 2005). Moreover, it was shown that OCT4 overexpression resulted in dedifferentiation of melanoma cells into "cancer stem-cells" (CSC). These cells acquired the 
typical CSC characteristics such as the ability to form tumor spheroids and the ability to resist chemotherapeutics and hypoxia (Kumar et al., 2012). Moreover, we found that OCT4 and moderate levels of BCL9-2 are co-expressed in mouse embryonic stem cells (mESCs) (data not shown). Probably, also in cancer cells, which to some extent recapitulate similar processes as during early embryogenesis, OCT4 and BCL9-2 may contribute to stem-cell like properties, either in cooperation or independently of each other. Future studies will therefore be extended to mouse embryonic stem-cells to analyze their function in stem cells. To this end, the expression of BCL9-2 in pluripotent and differentiating mESCs will be examined. Furthermore the phenotypes of mESCs following overexpression or downregulation of BCL9-2 and their pluripotency and differentiation potential will be analyzed. On the other hand, it is important to investigate whether OCT4 overexpression or downregulation in colon cancer cell lines with different levels of BCL9-2 changes their stem cell characteristics, motility and ability to proliferate.

\subsubsection{CDX1 and CDX2 expression is regulated by BCL9-2 through newly identified SP1 binding elements in their proximal promoter}

BCL9-2 does not contain a DNA-binding motif and is therefore not able to bind to cisregulatory elements in the promoters of target genes itself (Brembeck et al., 2004). Therefore the co-activator requires an adaptor protein, which links BCL9-2 to the transcription machinery where it exerts transcriptional activating functions. Here we describe a novel interaction for this factor. BCL9-2 binds to specificity protein 1 (SP1) transcription factors (TF) in colon cancer cell lines, while this interaction was not detected for Pygopus 2. Thus, Pygopus 2 plays a role in the BCL9-2 dependent activation of target genes but appears to be dispensable for BCL9-2/SP1 complex formation.

In addition, multiple SP1 binding elements in the proximal promoters of CDX1 and CDX2 were identified in this work. Moreover, expression of CDX2 was independent of the presence of a TATA box or even a TATA like element. Therefore it is likely that the initiation of transcription is mediated trough GC-rich elements located closely to the transcription start site, as shown for other TATA-less genes (Davie et al., 2008; Lu and Archer, 2010;Singh et al., 2012;Suske, 1999). Investigations regarding the transcriptional regulation of the CDX2 gene primarily focus general transcription factors, which activate the expression of CDX2 such as FGF signaling through SMADs (Barros et al., 2008; Camilo et al., 2012). So far, studies investigating the TATAless transcriptional activation of the CDX2 gene were still missing. We did not found common 
elements in TATA-less promoters such as downstream core promoter elements, TBIID recognition element or initiator elements in promoter of the CDX2 gene. However, our study revealed that the activity of CDX1 and CDX2 Luciferase reporters containing the proximal promoters of the genes were highly dependent on SP1-binding elements (BEs). Moreover, the very proximal SP1 binding elements close to the transcription start site were essential for the BCL9-2-dependent transcriptional regulation of both reporters. These results suggest that SP1 in dependence of BCL9-2 initiate the transcription of CDX1 and CDX2 genes.

Our results indicate that BCL9-2 exerts co-activatory functions through binding to SP1 and thereby to the DNA of target genes. Moreover, the transcriptional regulation of CDX1 and CDX2 by BCL9-2/SP1 appeared to be independent of ß-catenin, since knockdown of the protein had no effect on CDX reporter activity.

Further investigations will determine whether SP1/BCL9-2 complex formation is specific for BCL9-2 or if also its homologue BCL9 binds to SP1. To proof the SP1-mediated recruitment of BCL9-2 to specific cis-acting elements in the promoters of CDX1, CDX2 and other target genes, chromatin-immunoprecipitation (ChIP) and electrophoretic mobility shift assays (EMSA) will be performed. These studies will further compare known Wnt-responsive elements bound by ßcatenin (WRE) and putative BCL9-2 bound elements in the promoters of these target genes.

Interestingly, LEF/TCF transcription factors had been shown to interact with SP1/SP5 in different biological systems. For example, as previously shown for BCL9-2, SP5 is important for Wnt8-dependent transcriptional regulation of Wnt/B-catenin target genes in zebrafish mesoderm specification. In this regard, SP5 dependent transcription might be dependent on BCL9-2. Possibly, SP1 binding elements are further important to mediate the BCL9-2 dependent transcriptional regulation of target genes in dependency of Wnt responsive elements, which are bound by LEF/TCF in intestinal carcinogenesis (Figure 40, left). Since the impact of SP/LEF/TCF complexes on gene transcription differs in dependence on the genetic and biological background, detailed analyses in colon cancers are necessary to determine the consequence of the putative SP1/BCL9-2/LEF/TCF complex formation.

To identify a BCL9-2 protein domain, which is responsible for the binding of SP1, binding of the SP transcription factors to different truncated versions of BCL9-2 will be analyzed. These analyses will provide information about the dependency of this complex formation on different domains in the BCL9 proteins, e.g. whether ß-catenin binding or the BCL9-2 Cterminus is required for an interaction with SP1. The C-terminus of BCL9 was previously described by Sustmann and colleagues to harbor a transactivation domain (Sustmann et al., 
2008). The three C-terminal domains of BCL9-2 and BCL9 show high similarities (Brembeck et al., 2004;Kramps et al., 2002) and are important for the co-activatory function of BCL9 and BCL9-2 in Wnt/B-catenin signaling (Brembeck et al., 2004;Sustmann et al., 2008). In addition, the C-terminal domains might play a role in the SP1/BCL9-2 complex mediated transcriptional activation due to the facilitation of the SP1-BCL9-2 interaction. Moreover, until now it is unclear whether SP1/BCL9-2 complexes are a feature of cancer cells or a general mechanism in many cell types including untransformed, normal epithelial cells. Thus, future studies will be extended to other cell types such as additional breast cancer-, colon cancer- and untransformed- cell lines like the human embryonic kidney cell line HEK293.

SP1 transcription factors are ubiquitously expressed in all mammalian cells and belong to the highly conserved Protein/Krüppel-like Factor (SP/KLF) transcription factor family. SP/KLF factors contain a DNA binding domain and three Zink finger domains (Brandeis et al., 1994;Davie et al., 2008;Smale et al., 1990). SP1 proteins contain two trans-activation domains and serine/threonine-rich subregions which can be post-translational modified which primary results in inhibited transcriptional activity (Yang et al., 2001). The protein structure of SP1/KLF proteins is highly conserved. Thus, BCL9-2 might also bind to other SP1/KLF proteins. The closest structural relative of SP1 is SP3, which recognizes the same promoter binding elements. Like BCL9-2 knockout mice, SP1 knockout mice are embryonic lethal at E10.5, whereas SP3 knockout causes postnatal death suggesting a more prominent role of SP1 during embryonic development. In addition, BCL9-2 and SP1 knockout resulted in placental defects (Kruger et al., 2007). Interestingly, similar to BCL9-2, SP1 and SP3 are overexpressed in breast, colon and other tumor entities (Li et al., 2010).

Future studies will investigate whether BCL9 proteins interact with other SP related such as KLF4. Like OCT4, KLF4 is an important transcription factor in mouse embryonic stem-cells (ESCs). In addition, many studies claimed a dependency of SP1-TF and KLF4-TF on each other regarding their potential to activate transcription (Black et al., 2001;Brembeck et al., 2000;Shie et al., 2000). For example, CyclinD1 gene regulation is mediated by KLF4 which is in part dependent by binding to SP1 in gastric cancer cells (Shie et al., 2000). In addition, Brembeck et al. showed that the K19 promoter is active in gastrointestinal cancer cells through the transcriptional regulation by KLF4 and SP1. This study further claimed that the functional interaction of ubiquitous (SP1) and tissue-restricted (KLF4) transcription factors determines tissue- and neoplasm-specific patterns of gene expression (Brembeck et al., 2000). In this regard, BCL9-2 might play a tissue specific role for the induction of SP/KLF target genes. Thus, BCL9-2 transcriptional activity is dependent on SP1 and probably on OCT4. Therefore, we will 
address the question whether KLF4 plays a BCL9-2 dependent role in colon cancer and stemcell maintenance.

Moreover, SP1, SP3 as well as KLF4 transcription factors have been shown to be implicated in the transcriptional regulation of pluripotency genes such as OCT4 and NANOG in mESCs (Pesce et al., 1999;Wu and Yao, 2006). Possibly, BCL9-2 and SP proteins might further function in a complex in embryonic stem cells which activate pluripotency genes in ESCs (see section 5.4.2). BCL9-2 might additionally exert a function in a SP1/SP3 mediated co-regulation of OCT4 in cancer cells. BCL9-2 and SP transcriptional complexes in colon cancers might contribute to the expression of genes which are essential for the development of stem-cell like features. As it was already shown for many BCL9-2 target genes, SP1 target gene products are also implicated in tumorigenesis where they act in different cancer-related processes like cell cycle progression/arrest, proliferation, invasion, metastasis and angiogenesis (Li et al., 2010). Subsequently, the SP1/BCL9-2 complex dependent transcription might activate the expression of cancer-related genes that trigger the oncogenic function of BCL9-2

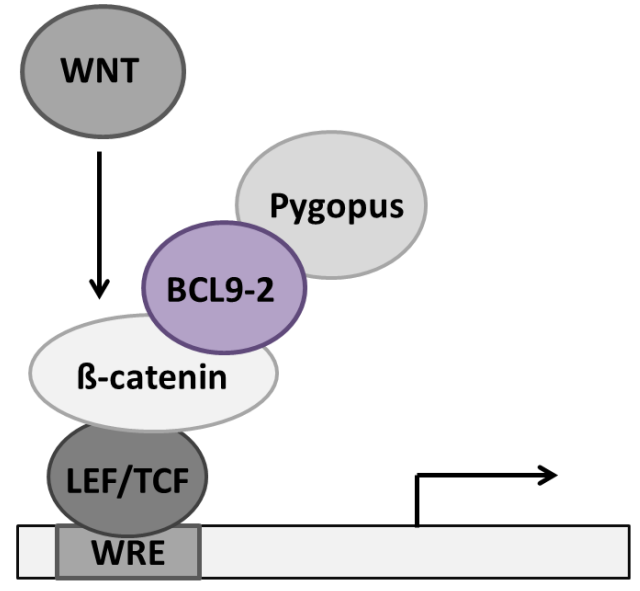

A. Wnt/ß-catenin target genes: Axin2, TCF1, EPHB2

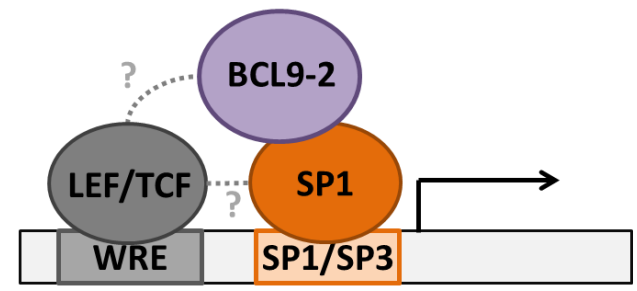

B. B-catenin independent genes e.g. CDX1, CDX2

Figure 40: BCL9-2 regulates canonical Wnt-target genes and $B$-catenin independent genes BCL9-2 is important for the regulation of (A) Wnt/B-catenin target gene and binds to LEF/TCF trough Bcatenin, which is activated by paracrine WNT signals. In this regard, BCL9-2 links Pygopus 2 to the complex. (B) BCL9-2 activates ß-catenin-independent target genes through complex formation with SP1 transcription factors. In addition BCL9-2 or/and SP1 might be recruited to LEF/TCF transcription factors and possibly exerts B-catenin-independent function trough Wnt-responsive elements (WRE) in the promoters of target genes. 


\section{Summary and Conclusion}

The function of B-cell CLL/lymphoma 9 protein (BCL9)/Legless in Drosophila has been investigated intensively (Kessler et al., 2009;Kramps et al., 2002;Townsley et al., 2004). However, the role of the vertebrate orthologs in Wnt/ß-catenin signaling and in further processes seems to be more complex than in Drosophila. This work investigated the ß-catenindependent and -independent functions of BCL9 and BCL9-2 in normal intestine and tumorigenesis.

BCL9 was expressed in all intestinal cell types and unchanged in colon cancer cells. In contrast, BCL9-2 protein expression was restricted to the villi in untransformed, normal intestines, and absent in the crypts where Wnt-signaling is active, indicating that BCL9-2 is dispensable for Wnt/ß-catenin signaling in intestinal homeostasis. However, BCL9-2 overexpression enhanced ß-catenin mediated transcription of a subset of target genes and correlated with the level of Wnt/B-catenin signaling activity in colon cancer cells. Moreover, BCL9-2, and partially BCL9, regulated the expression of ß-catenin independent genes which have been implicated in tumorigenesis. Moreover, BCL9-2 was already overexpressed in early stages of intestinal tumorigenesis and additionally elevated in approximately $90 \%$ of human adenocarcinomas independent of metastases formation. Ectopic overexpression of BCL9-2 in the intestine of transgenic K19-BCL9-2;APC ${ }^{\mathrm{Min} /+}$ mice led to increased adenoma formation accompanied with local invasion which resulted in reduced survival. In cancer cells BCL9-2 associates with specific protein 1 (SP1). Moreover, BCL9-2 mediated activation of CDX1 and CDX2 reporter gene transcription was dependent on SP1-binding elements in their proximal promoters in colon cancer cell lines.

In conclusion, this work demonstrates that BCL9-2 promotes early phases of intestinal tumor development and contributes to the progression of tumors into invasive carcinomas. These features are obviously triggered by the transcriptional regulation of partially ß-cateninindependent target genes. Moreover, BCL9-2 mediates its co-activatory function ß-cateninindependently through binding to SP1 transcription factors and thereby to the promoters of target genes which are implicated in early phases of tumor progression.

Future studies will also focus the implication of other SP/KLF transcription factors in the BCL9-2 mediated transcriptional regulation of target genes. In addition, the recruitment of BCL9-2 to ß-catenin-dependent and -independent cis-acting elements will be proofed in cancer- and untransformed- cell lines. 


\section{References}

1. Abdul Khalek FJ, Gallicano GI, and Mishra L (2010) Colon cancer stem cells. Gastrointest Cancer Res, S16-S23.

2. Adachi $\mathrm{N}$ and Lieber MR (2002) Bidirectional gene organization: a common architectural feature of the human genome. Cell, 109, 807-809.

3. Adachi S, Jigami T, Yasui T, Nakano T, Ohwada S, Omori Y, Sugano S, Ohkawara B, Shibuya H, Nakamura T, and Akiyama T (2004) Role of a BCL9-related beta-cateninbinding protein, $\mathrm{B} 9 \mathrm{~L}$, in tumorigenesis induced by aberrant activation of Wnt signaling. Cancer Res, 64, 8496-8501.

4. Alberici P and Fodde $R$ (2006) The role of the APC tumor suppressor in chromosomal instability. Genome Dyn, 1, 149-170.

5. Andrews PG, He Z, Popadiuk C, and Kao KR (2009) The transcriptional activity of Pygopus is enhanced by its interaction with CAMP-response-element-binding protein (CREB)-binding protein. Biochem J, 422, 493-501.

6. Arce L, Yokoyama NN, and Waterman ML (2006) Diversity of LEF/TCF action in development and disease. Oncogene, 25, 7492-7504.

7. Bakaris S, Cetinkaya A, Ezberci F, and Ekerbicer H (2008) Expression of homeodomain protein CDX2 in colorectal adenoma and adenocarcinoma. Histol Histopathol, 23, 1043-1047.

8. Barker N, Hurlstone A, Musisi H, Miles A, Bienz M, and Clevers H (2001) The chromatin remodelling factor $\mathrm{Brg}-1$ interacts with beta-catenin to promote target gene activation. EMBO J, 20, 4935-4943.

9. Barker N, Ridgway RA, van Es JH, van de Wetering $M$, Begthel $H$, van den Born $M$, Danenberg E, Clarke AR, Sansom OJ, and Clevers H (2009) Crypt stem cells as the cellsof-origin of intestinal cancer. Nature, 457, 608-611.

10. Barros R, Pereira B, Duluc I, Azevedo M, Mendes N, Camilo V, Jacobs RJ, Paulo P, Santos-Silva F, van S, I, van den Brink GR, David L, Freund JN, and Almeida R (2008) Key elements of the BMP/SMAD pathway co-localize with CDX2 in intestinal metaplasia and regulate CDX2 expression in human gastric cell lines. J Pathol, 215, 411-420.

11. Batlle E, Henderson JT, Beghtel H, van den Born MM, Sancho E, Huls G, Meeldijk J, Robertson J, van de Wetering M, Pawson T, and Clevers H (2002) Beta-catenin and TCF mediate cell positioning in the intestinal epithelium by controlling the expression of EphB/ephrinB. Cell, 111, 251-263.

12. Bauer A, Huber O, and Kemler R (1998) Pontin52, an interaction partner of betacatenin, binds to the TATA box binding protein. Proc Natl Acad Sci U S A, 95, 1478714792. 
13. Behrens J, von Kries JP, Kuhl M, Bruhn L, Wedlich D, Grosschedl R, and Birchmeier W (1996) Functional interaction of beta-catenin with the transcription factor LEF-1. Nature, 382, 638-642.

14. Belenkaya TY, Han C, Standley HJ, Lin X, Houston DW, Heasman J, and Lin X (2002) pygopus Encodes a nuclear protein essential for wingless/Wnt signaling. Development, 129, 4089-4101.

15. Bhat AA, Sharma A, Pope J, Krishnan $M$, Washington $M K$, Singh AB, and Dhawan $P$ (2012) Caudal homeobox protein $\mathrm{Cdx}-2$ cooperates with Wnt pathway to regulate claudin-1 expression in colon cancer cells. PLoS One, 7, e37174.

16. Bienz M and Clevers H (2000) Linking colorectal cancer to Wnt signaling. Cell, 103, 311320.

17. Blache $P$, van de Wetering $M$, Duluc I, Domon C, Berta P, Freund JN, Clevers $H$, and Jay $P(2004)$ SOX9 is an intestine crypt transcription factor, is regulated by the Wnt pathway, and represses the CDX2 and MUC2 genes. J Cell Biol, 166, 37-47.

18. Black AR, Black JD, and Azizkhan-Clifford J (2001) Sp1 and kruppel-like factor family of transcription factors in cell growth regulation and cancer. J Cell Physiol, 188, 143-160.

19. Blackwood EM and Kadonaga JT (1998) Going the distance: a current view of enhancer action. Science, 281, 60-63.

20. Bonhomme C, Duluc I, Martin E, Chawengsaksophak K, Chenard MP, Kedinger M, Beck F, Freund JN, and Domon-Dell C (2003) The Cdx2 homeobox gene has a tumour suppressor function in the distal colon in addition to a homeotic role during gut development. Gut, 52, 1465-1471.

21. Boras-Granic K and Wysolmerski JJ (2008) Wnt signaling in breast organogenesis. Organogenesis, 4, 116-122.

22. Brack AS, Conboy IM, Conboy MJ, Shen J, and Rando TA (2008) A temporal switch from notch to Wnt signaling in muscle stem cells is necessary for normal adult myogenesis. Cell Stem Cell, 2, 50-59.

23. Brack AS, Murphy-Seiler F, Hanifi J, Deka J, Eyckerman S, Keller C, Aguet M, and Rando TA (2009) BCL9 is an essential component of canonical Wnt signaling that mediates the differentiation of myogenic progenitors during muscle regeneration. Dev Biol, 335, 93105.

24. Bradford MM (1976) A rapid and sensitive method for the quantitation of microgram quantities of protein utilizing the principle of protein-dye binding. Anal Biochem, 72, 248-254.

25. Brandeis M, Frank D, Keshet I, Siegfried Z, Mendelsohn M, Nemes A, Temper V, Razin $A$, and Cedar H (1994) Sp1 elements protect a CpG island from de novo methylation. Nature, 371, 435-438.

26. Brannon M, Brown JD, Bates R, Kimelman D, and Moon RT (1999) XCtBP is a XTcf-3 corepressor with roles throughout Xenopus development. Development, 126, 3159-3170. 
27. Brattain MG, Fine WD, Khaled FM, Thompson J, and Brattain DE (1981) Heterogeneity of malignant cells from a human colonic carcinoma. Cancer Res, 41, 1751-1756.

28. Brembeck FH, Moffett J, Wang TC, and Rustgi AK (2001) The keratin 19 promoter is potent for cell-specific targeting of genes in transgenic mice. Gastroenterology, 120, 1720-1728.

29. Brembeck FH, Rosario M, and Birchmeier W (2006) Balancing cell adhesion and Wnt signaling, the key role of beta-catenin. Curr Opin Genet Dev, 16, 51-59.

30. Brembeck FH and Rustgi AK (2000) The tissue-dependent keratin 19 gene transcription is regulated by GKLF/KLF4 and Sp1. J Biol Chem, 275, 28230-28239.

31. Brembeck FH, Schwarz-Romond T, Bakkers J, Wilhelm S, Hammerschmidt $M$, and Birchmeier W (2004) Essential role of BCL9-2 in the switch between beta-catenin's adhesive and transcriptional functions. Genes Dev, 18, 2225-2230.

32. Brembeck FH, Wiese $M$, Zatula N, Grigoryan T, Dai Y, Fritzmann J, and Birchmeier W (2011) BCL9-2 promotes early stages of intestinal tumor progression.

Gastroenterology, 141, 1359-70, 1370.

33. Bucher P (1990) Weight matrix descriptions of four eukaryotic RNA polymerase II promoter elements derived from 502 unrelated promoter sequences. J Mol Biol, 212, 563-578.

34. Buller NV, Rosekrans SL, Westerlund J, and van den Brink GR (2012) Hedgehog signaling and maintenance of homeostasis in the intestinal epithelium. Physiology (Bethesda ), 27, 148-155.

35. Burley SK and Roeder RG (1996) Biochemistry and structural biology of transcription factor IID (TFIID). Annu Rev Biochem, 65, 769-799.

36. Caldwell CM and Kaplan KB (2009) The role of APC in mitosis and in chromosome instability. Adv Exp Med Biol, 656, 51-64.

37. Camilo V, Barros R, Sousa S, Magalhaes AM, Lopes T, Mario SA, Pereira T, Figueiredo C, David L, and Almeida R (2012) Helicobacter pylori and the BMP pathway regulate CDX2 and SOX2 expression in gastric cells. Carcinogenesis, 33, 1985-1992.

38. Cavallo RA, Cox RT, Moline MM, Roose J, Polevoy GA, Clevers H, Peifer M, and Bejsovec A (1998) Drosophila Tcf and Groucho interact to repress Wingless signalling activity. Nature, 395, 604-608.

39. Chen CM and Struhl G (1999) Wingless transduction by the Frizzled and Frizzled2 proteins of Drosophila. Development, 126, 5441-5452.

40. Chen G, Fernandez J, Mische S, and Courey AJ (1999) A functional interaction between the histone deacetylase Rpd3 and the corepressor groucho in Drosophila development. Genes Dev, 13, 2218-2230.

41. Chen J, Luo Q, Yuan Y, Huang X, Cai W, Li C, Wei T, Zhang L, Yang M, Liu Q, Ye G, Dai X, and Li B (2010) Pygo2 associates with MLL2 histone methyltransferase and GCN5 histone acetyltransferase complexes to augment Wnt target gene expression and breast cancer stem-like cell expansion. Mol Cell Biol, 30, 5621-5635. 
42. Chen L, Yabuuchi A, Eminli S, Takeuchi A, Lu CW, Hochedlinger K, and Daley GQ (2009) Cross-regulation of the Nanog and Cdx2 promoters. Cell Res, 19, 1052-1061.

43. Chen TR, Drabkowski D, Hay RJ, Macy M, and Peterson W, Jr. (1987) WiDr is a derivative of another colon adenocarcinoma cell line, HT-29. Cancer Genet Cytogenet, 27, 125-134.

44. Chinnadurai G (2002) CtBP, an unconventional transcriptional corepressor in development and oncogenesis. Mol Cell, 9, 213-224.

45. Choi KJ, Piao YJ, Lim MJ, Kim JH, Ha J, Choe W, and Kim SS (2007) Overexpressed cyclophilin A in cancer cells renders resistance to hypoxia- and cisplatin-induced cell death. Cancer Res, 67, 3654-3662.

46. Clements D, Taylor HC, Herrmann BG, and Stott D (1996) Distinct regulatory control of the Brachyury gene in axial and non-axial mesoderm suggests separation of mesoderm lineages early in mouse gastrulation. Mech Dev, 56, 139-149.

47. Crosnier C, Stamataki D, and Lewis J (2006) Organizing cell renewal in the intestine: stem cells, signals and combinatorial control. Nat Rev Genet, 7, 349-359.

48. Daniels DL and Weis WI (2005) Beta-catenin directly displaces Groucho/TLE repressors from Tcf/Lef in Wnt-mediated transcription activation. Nat Struct Mol Biol, 12, 364371.

49. Davie JR, He S, Li L, Sekhavat A, Espino P, Drobic B, Dunn KL, Sun JM, Chen HY, Yu J, Pritchard S, and Wang X (2008) Nuclear organization and chromatin dynamics--Sp1, Sp3 and histone deacetylases. Adv Enzyme Regul, 48, 189-208.

50. de la Roche M, Rutherford TJ, Gupta D, Veprintsev DB, Saxty B, Freund SM, and Bienz $M$ (2012a) An intrinsically labile alpha-helix abutting the BCL9-binding site of betacatenin is required for its inhibition by carnosic acid. Nat Commun, 3, 680.

51. de la Roche M, Rutherford TJ, Gupta D, Veprintsev DB, Saxty B, Freund SM, and Bienz $M(2012 b)$ An intrinsically labile alpha-helix abutting the BCL9-binding site of betacatenin is required for its inhibition by carnosic acid. Nat Commun, 3, 680.

52. de la Roche M, Worm J, and Bienz M (2008) The function of BCL9 in Wnt/beta-catenin signaling and colorectal cancer cells. BMC Cancer, 8, 199.

53. Deka J, Wiedemann N, Anderle P, Murphy-Seiler F, Bultinck J, Eyckerman S, Stehle JC, Andre S, Vilain N, Zilian O, Robine S, Delorenzi M, Basler K, and Aguet M (2010) $\mathrm{Bc} 19 / \mathrm{Bc} \mid 9 \mathrm{l}$ are critical for Wnt-mediated regulation of stem cell traits in colon epithelium and adenocarcinomas. Cancer Res, 70, 6619-6628.

54. Dexter DL, Barbosa JA, and Calabresi P (1979) N,N-dimethylformamide-induced alteration of cell culture characteristics and loss of tumorigenicity in cultured human colon carcinoma cells. Cancer Res, 39, 1020-1025.

55. Dhawan P, Singh AB, Deane NG, No Y, Shiou SR, Schmidt C, Neff J, Washington MK, and Beauchamp RD (2005) Claudin-1 regulates cellular transformation and metastatic behavior in colon cancer. J Clin Invest, 115, 1765-1776. 
56. Di Guglielmo MD, Park J, Schulz S, and Waldman SA (2001) Nucleotide requirements for CDX2 binding to the cis promoter element mediating intestine-specific expression of guanylyl cyclase C. FEBS Lett, 507, 128-132.

57. Di TP, Moretti S, Xenarios I, Orobitg M, Montanyola A, Chang JM, Taly JF, and Notredame C (2011) T-Coffee: a web server for the multiple sequence alignment of protein and RNA sequences using structural information and homology extension. Nucleic Acids Res, 39, W13-W17.

58. Diecke S, Quiroga-Negreira A, Redmer T, and Besser D (2008) FGF2 signaling in mouse embryonic fibroblasts is crucial for self-renewal of embryonic stem cells. Cells Tissues Organs, 188, 52-61.

59. Dikovskaya D, Schiffmann D, Newton IP, Oakley A, Kroboth K, Sansom O, Jamieson TJ, Meniel V, Clarke A, and Nathke IS (2007) Loss of APC induces polyploidy as a result of a combination of defects in mitosis and apoptosis. J Cell Biol, 176, 183-195.

60. Drewinko B, Romsdahl MM, Yang LY, Ahearn MJ, and Trujillo JM (1976) Establishment of a human carcinoembryonic antigen-producing colon adenocarcinoma cell line. Cancer Res, 36, 467-475.

61. Ee HC, Erler T, Bhathal PS, Young GP, and James RJ (1995) Cdx-2 homeodomain protein expression in human and rat colorectal adenoma and carcinoma. Am J Pathol, 147, 586-592.

62. Efstathiou JA, Liu D, Wheeler JM, Kim HC, Beck NE, Ilyas M, Karayiannakis AJ, Mortensen NJ, Kmiot W, Playford RJ, Pignatelli M, and Bodmer WF (1999) Mutated epithelial cadherin is associated with increased tumorigenicity and loss of adhesion and of responsiveness to the motogenic trefoil factor 2 in colon carcinoma cells. Proc Natl Acad Sci U S A, 96, 2316-2321.

63. Elyada E, Pribluda A, Goldstein RE, Morgenstern Y, Brachya G, Cojocaru G, Snir-Alkalay I, Burstain I, Haffner-Krausz R, Jung S, Wiener Z, Alitalo K, Oren M, Pikarsky E, and BenNeriah Y (2011) CKlalpha ablation highlights a critical role for p53 in invasiveness control. Nature, 470, 409-413.

64. Fearon ER and Vogelstein B (1990) A genetic model for colorectal tumorigenesis. Cell, 61, 759-767.

65. Feng XH, Lin X, and Derynck R (2000) Smad2, Smad3 and Smad4 cooperate with Sp1 to induce p15(Ink4B) transcription in response to TGF-beta. EMBO J, 19, 5178-5193.

66. Feng Y, Dai X, Li X, Wang H, Liu J, Zhang J, Du Y, and Xia L (2012) EGF signalling pathway regulates colon cancer stem cell proliferation and apoptosis. Cell Prolif, 45, 413-419.

67. Fiedler M, Sanchez-Barrena MJ, Nekrasov M, Mieszczanek J, Rybin V, Muller J, Evans P, and Bienz M (2008) Decoding of methylated histone H3 tail by the Pygo-BCL9 Wnt signaling complex. Mol Cell, 30, 507-518.

68. Fisher AL and Caudy M (1998) Groucho proteins: transcriptional corepressors for specific subsets of DNA-binding transcription factors in vertebrates and invertebrates. Genes Dev, 12, 1931-1940. 
69. Fleige S, Walf V, Huch S, Prgomet C, Sehm J, and Pfaffl MW (2006) Comparison of relative $m R N A$ quantification models and the impact of RNA integrity in quantitative real-time RT-PCR. Biotechnol Lett, 28, 1601-1613.

70. Fodde R and Brabletz $T$ (2007) Wnt/beta-catenin signaling in cancer stemness and malignant behavior. Curr Opin Cell Biol, 19, 150-158.

71. Fodde R, Smits R, and Clevers H (2001) APC, signal transduction and genetic instability in colorectal cancer. Nat Rev Cancer, 1, 55-67.

72. Fogh J, Fogh JM, and Orfeo T (1977a) One hundred and twenty-seven cultured human tumor cell lines producing tumors in nude mice. J Natl Cancer Inst, 59, 221-226.

73. Fogh J, Wright WC, and Loveless JD (1977b) Absence of HeLa cell contamination in 169 cell lines derived from human tumors. J Nat/ Cancer Inst, 58, 209-214.

74. Fritzmann J, Morkel M, Besser D, Budczies J, Kosel F, Brembeck FH, Stein U, Fichtner I, Schlag PM, and Birchmeier W (2009) A colorectal cancer expression profile that includes transforming growth factor beta inhibitor BAMBI predicts metastatic potential. Gastroenterology, 137, 165-175.

75. Fujimura N, Vacik T, Machon O, Vlcek C, Scalabrin S, Speth M, Diep D, Krauss S, and Kozmik Z (2007) Wnt-mediated down-regulation of Sp1 target genes by a transcriptional repressor Sp5. J Biol Chem, 282, 1225-1237.

76. Gadue P, Huber TL, Paddison PJ, and Keller GM (2006) Wnt and TGF-beta signaling are required for the induction of an in vitro model of primitive streak formation using embryonic stem cells. Proc Natl Acad Sci U S A, 103, 16806-16811.

77. Gao N, White P, and Kaestner KH (2009) Establishment of intestinal identity and epithelial-mesenchymal signaling by Cdx2. Dev Cell, 16, 588-599.

78. Garabedian EM, Roberts LJ, McNevin MS, and Gordon JI (1997) Examining the role of Paneth cells in the small intestine by lineage ablation in transgenic mice. J Biol Chem, 272, 23729-23740.

79. Gashaw I, Dushaj O, Behr R, Biermann K, Brehm R, Rubben H, Grobholz R, Schmid KW, Bergmann $M$, and Winterhager $E$ (2007) Novel germ cell markers characterize testicular seminoma and fetal testis. Mol Hum Reprod, 13, 721-727.

80. Gaspar C and Fodde R (2004) APC dosage effects in tumorigenesis and stem cell differentiation. Int J Dev Biol, 48, 377-386.

81. Golan T, Yaniv A, Bafico A, Liu G, and Gazit A (2004) The human Frizzled 6 (HFz6) acts as a negative regulator of the canonical Wnt. beta-catenin signaling cascade. J Biol Chem, 279, 14879-14888.

82. Graham FL, Smiley J, Russell WC, and Nairn R (1977) Characteristics of a human cell line transformed by DNA from human adenovirus type 5. J Gen Virol, 36, 59-74.

83. Greaves S, Sanson B, White P, and Vincent JP (1999) A screen for identifying genes interacting with armadillo, the Drosophila homolog of beta-catenin. Genetics, 153, 1753-1766. 
84. Gregorieff $A$ and Clevers $H(2005)$ Wnt signaling in the intestinal epithelium: from endoderm to cancer. Genes Dev, 19, 877-890.

85. Gregorieff A, Grosschedl $\mathrm{R}$, and Clevers $\mathrm{H}$ (2004) Hindgut defects and transformation of the gastro-intestinal tract in Tcf4(-/-)/Tcf1(-/-) embryos. EMBO J, 23, 1825-1833.

86. Grigoryan T, Wend P, Klaus A, and Birchmeier W (2008) Deciphering the function of canonical Wnt signals in development and disease: conditional loss- and gain-offunction mutations of beta-catenin in mice. Genes Dev, 22, 2308-2341.

87. Gu B, Sun P, Yuan Y, Moraes RC, Li A, Teng A, Agrawal A, Rheaume C, Bilanchone V, Veltmaat JM, Takemaru K, Millar S, Lee EY, Lewis MT, Li B, and Dai X (2009) Pygo2 expands mammary progenitor cells by facilitating histone $\mathrm{H} 3 \mathrm{~K} 4$ methylation. J Cell Biol, 185, 811-826.

88. Gumbiner BM (2000) Regulation of cadherin adhesive activity. J Cell Biol, 148, 399-404.

89. Guo RJ, Suh ER, and Lynch JP (2004) The role of Cdx proteins in intestinal development and cancer. Cancer Biol Ther, 3, 593-601.

90. Habas R and Dawid IB (2005) Dishevelled and Wnt signaling: is the nucleus the final frontier? J Biol, 4, 2.

91. Hafner C, Schmitz G, Meyer S, Bataille F, Hau P, Langmann T, Dietmaier W, Landthaler $M$, and Vogt $T$ (2004) Differential gene expression of Eph receptors and ephrins in benign human tissues and cancers. Clin Chem, 50, 490-499.

92. Hall PA, Coates PJ, Ansari B, and Hopwood D (1994) Regulation of cell number in the mammalian gastrointestinal tract: the importance of apoptosis. J Cell Sci, 107 ( Pt 12), 3569-3577.

93. Hampf M and Gossen M (2006) A protocol for combined Photinus and Renilla luciferase quantification compatible with protein assays. Anal Biochem, 356, 94-99.

94. Han J, Gao B, Jin X, Xu Z, Li Z, Sun Y, and Song B (2012) Small interfering RNA-mediated downregulation of beta-catenin inhibits invasion and migration of colon cancer cells in vitro. Med Sci Monit, 18, BR273-BR280.

95. Haramis AP, Begthel $\mathrm{H}$, van den Born $\mathrm{M}$, van EJ, Jonkheer $\mathrm{S}$, Offerhaus GJ, and Clevers $\mathrm{H}$ (2004) De novo crypt formation and juvenile polyposis on BMP inhibition in mouse intestine. Science, 303, 1684-1686.

96. Hardwick JC, van den Brink GR, Bleuming SA, Ballester I, Van Den Brande JM, Keller JJ, Offerhaus GJ, van Deventer SJ, and Peppelenbosch MP (2004) Bone morphogenetic protein 2 is expressed by, and acts upon, mature epithelial cells in the colon. Gastroenterology, 126, 111-121.

97. Hartenstein V, Takashima S, and Adams KL (2010) Conserved genetic pathways controlling the development of the diffuse endocrine system in vertebrates and Drosophila. Gen Comp Endocrinol, 166, 462-469.

98. Hay DC, Sutherland L, Clark J, and Burdon T (2004) Oct-4 knockdown induces similar patterns of endoderm and trophoblast differentiation markers in human and mouse embryonic stem cells. Stem Cells, 22, 225-235. 
99. He X, Semenov M, Tamai K, and Zeng X (2004a) LDL receptor-related proteins 5 and 6 in Wnt/beta-catenin signaling: arrows point the way. Development, 131, 1663-1677.

100. He XC, Zhang J, Tong WG, Tawfik O, Ross J, Scoville DH, Tian Q, Zeng X, He X, Wiedemann LM, Mishina Y, and Li L (2004b) BMP signaling inhibits intestinal stem cell self-renewal through suppression of Wnt-beta-catenin signaling. Nat Genet, 36, 11171121.

101. Hecht A, Litterst CM, Huber O, and Kemler R (1999) Functional characterization of multiple transactivating elements in beta-catenin, some of which interact with the TATA-binding protein in vitro. J Biol Chem, 274, 18017-18025.

102. Hecht A, Vleminckx K, Stemmler MP, van RF, and Kemler R (2000) The p300/CBP acetyltransferases function as transcriptional coactivators of beta-catenin in vertebrates. $E M B O \mathrm{~J}, 19,1839-1850$.

103. Hicks GR and Raikhel NV (1995) Protein import into the nucleus: an integrated view. Annu Rev Cell Dev Biol, 11, 155-188.

104. Higgins KJ, Abdelrahim M, Liu S, Yoon K, and Safe S (2006a) Regulation of vascular endothelial growth factor receptor-2 expression in pancreatic cancer cells by $\mathrm{Sp}$ proteins. Biochem Biophys Res Commun, 345, 292-301.

105. Higgins KJ, Liu S, Abdelrahim M, Yoon K, Vanderlaag K, Porter W, Metz RP, and Safe S (2006b) Vascular endothelial growth factor receptor-2 expression is induced by 17beta-estradiol in ZR-75 breast cancer cells by estrogen receptor alpha/Sp proteins. Endocrinology, 147, 3285-3295.

106. Hinoi T, Loda M, and Fearon ER (2003) Silencing of CDX2 expression in colon cancer via a dominant repression pathway. J Biol Chem, 278, 44608-44616.

107. Hinoi T, Tani M, Lucas PC, Caca K, Dunn RL, Macri E, Loda M, Appelman HD, Cho KR, and Fearon ER (2001) Loss of CDX2 expression and microsatellite instability are prominent features of large cell minimally differentiated carcinomas of the colon. Am J Pathol, 159, 2239-2248.

108. Hochedlinger K, Yamada Y, Beard C, and Jaenisch R (2005) Ectopic expression of Oct-4 blocks progenitor-cell differentiation and causes dysplasia in epithelial tissues. Cell, 121, 465-477.

109. Holmberg J, Genander M, Halford MM, Anneren C, Sondell M, Chumley MJ, Silvany RE, Henkemeyer M, and Frisen J (2006) EphB receptors coordinate migration and proliferation in the intestinal stem cell niche. Cell, 125, 1151-1163.

110. Hryniuk A, Grainger S, Savory JG, and Lohnes D (2012) Cdx function is required for maintenance of intestinal identity in the adult. Dev Biol, 363, 426-437.

111. Hu Y, Kazenwadel J, and James R (1993) Isolation and characterization of the murine homeobox gene $\mathrm{Cdx}-1$. Regulation of expression in intestinal epithelial cells. J Biol Chem, 268, 27214-27225.

112. Huelsken J and Birchmeier W (2001) New aspects of Wnt signaling pathways in higher vertebrates. Curr Opin Genet Dev, 11, 547-553. 
113. Hussein SM, Duff EK, and Sirard C (2003) Smad4 and beta-catenin co-activators functionally interact with lymphoid-enhancing factor to regulate graded expression of Msx2. J Biol Chem, 278, 48805-48814.

114. Ichii S, Nagase H, Mori T, Baba S, and Nakamura Y (1993) A novel mutation of the APC (adenomatous polyposis coli) gene in a familial adenomatous polyposis (FAP) patient and presymptomatic diagnosis using PCR. Hum Mol Genet, 2, 597.

115. Ikeda Y, Yamamoto J, Okamura M, Fujino T, Takahashi S, Takeuchi K, Osborne TF, Yamamoto TT, Ito S, and Sakai J (2001) Transcriptional regulation of the murine acetylCoA synthetase 1 gene through multiple clustered binding sites for sterol regulatory element-binding proteins and a single neighboring site for Sp1. J Biol Chem, 276, 34259-34269.

116. Ilyas M, Tomlinson IP, Rowan A, Pignatelli M, and Bodmer WF (1997) Beta-catenin mutations in cell lines established from human colorectal cancers. Proc Natl Acad Sci U $S A, 94,10330-10334$.

117. Imai K, Takada N, Satoh N, and Satou Y (2000) (beta)-catenin mediates the specification of endoderm cells in ascidian embryos. Development, 127, 3009-3020.

118. Ishitani T, Ninomiya-Tsuji J, and Matsumoto K (2003) Regulation of lymphoid enhancer factor $1 / T$-cell factor by mitogen-activated protein kinase-related Nemo-like kinasedependent phosphorylation in Wnt/beta-catenin signaling. Mol Cell Biol, 23, 13791389.

119. James R, Erler T, and Kazenwadel J (1994) Structure of the murine homeobox gene cdx-2. Expression in embryonic and adult intestinal epithelium. J Biol Chem, 269, 15229-15237.

120. Jamora C, DasGupta R, Kocieniewski P, and Fuchs E (2003) Links between signal transduction, transcription and adhesion in epithelial bud development. Nature, 422, 317-322.

121. Jamora $C$ and Fuchs E (2002) Intercellular adhesion, signalling and the cytoskeleton. Nat Cell Biol, 4, E101-E108.

122. Javahery R, Khachi A, Lo K, Zenzie-Gregory B, and Smale ST (1994) DNA sequence requirements for transcriptional initiator activity in mammalian cells. Mol Cell Biol, 14, 116-127.

123. Jessen S, Gu B, and Dai X (2008) Pygopus and the Wnt signaling pathway: a diverse set of connections. Bioessays, 30, 448-456.

124. Jho EH, Zhang T, Domon C, Joo CK, Freund JN, and Costantini F (2002) Wnt/betacatenin/Tcf signaling induces the transcription of Axin2, a negative regulator of the signaling pathway. Mol Cell Biol, 22, 1172-1183.

125. Jia Z, Zhang J, Wei D, Wang L, Yuan P, Le X, Li Q, Yao J, and Xie K (2007) Molecular basis of the synergistic antiangiogenic activity of bevacizumab and mithramycin A. Cancer Res, 67, 4878-4885.

126. Jin T, Branch DR, Zhang X, Qi S, Youngson B, and Goss PE (1999) Examination of POU homeobox gene expression in human breast cancer cells. Int J Cancer, 81, 104-112. 
127. Jung M, Peterson H, Chavez L, Kahlem P, Lehrach H, Vilo J, and Adjaye J (2010) A data integration approach to mapping OCT4 gene regulatory networks operative in embryonic stem cells and embryonal carcinoma cells. PLoS One, 5, e10709.

128. Kadonaga JT, Carner KR, Masiarz FR, and Tjian R (1987) Isolation of cDNA encoding transcription factor Sp1 and functional analysis of the DNA binding domain. Cell, 51, 1079-1090.

129. Karalay O, Doberauer K, Vadodaria KC, Knobloch M, Berti L, Miquelajauregui A, Schwark M, Jagasia R, Taketo MM, Tarabykin V, Lie DC, and Jessberger S (2011) Prospero-related homeobox 1 gene (Prox1) is regulated by canonical Wnt signaling and has a stage-specific role in adult hippocampal neurogenesis. Proc Natl Acad Sci U SA, 108, 5807-5812.

130. Kel AE, Gossling E, Reuter I, Cheremushkin E, Kel-Margoulis OV, and Wingender $E$ (2003) MATCH: A tool for searching transcription factor binding sites in DNA sequences. Nucleic Acids Res, 31, 3576-3579.

131. Kemp C, Willems E, Abdo S, Lambiv L, and Leyns L (2005) Expression of all Wnt genes and their secreted antagonists during mouse blastocyst and postimplantation development. Dev Dyn, 233, 1064-1075.

132. Kessler R, Hausmann G, and Basler K (2009) The PHD domain is required to link Drosophila Pygopus to Legless/beta-catenin and not to histone H3. Mech Dev, 126, 752-759.

133. Kim IJ, Kang HC, Park JH, Shin Y, Ku JL, Lim SB, Park SY, Jung SY, Kim HK, and Park JG (2003) Development and applications of a beta-catenin oligonucleotide microarray: beta-catenin mutations are dominantly found in the proximal colon cancers with microsatellite instability. Clin Cancer Res, 9, 2920-2925.

134. Knosel T, Petersen S, Schwabe H, Schluns K, Stein U, Schlag PM, Dietel M, and Petersen I (2002) Incidence of chromosomal imbalances in advanced colorectal carcinomas and their metastases. Virchows Arch, 440, 187-194.

135. Kong D, Li Y, Wang Z, and Sarkar FH (2011) Cancer Stem Cells and Epithelial-toMesenchymal Transition (EMT)-Phenotypic Cells: Are They Cousins or Twins? Cancers (Basel), 3, 716-729.

136. Korinek V, Barker N, Moerer P, van DE, Huls G, Peters PJ, and Clevers H (1998a) Depletion of epithelial stem-cell compartments in the small intestine of mice lacking Tcf-4. Nat Genet, 19, 379-383.

137. Korinek V, Barker N, Morin PJ, van WD, de WR, Kinzler KW, Vogelstein B, and Clevers H (1997) Constitutive transcriptional activation by a beta-catenin-Tcf complex in APC-/colon carcinoma. Science, 275, 1784-1787.

138. Korinek V, Barker N, Willert K, Molenaar M, Roose J, Wagenaar G, Markman M, Lamers W, Destree O, and Clevers $H$ (1998b) Two members of the Tcf family implicated in Wnt/beta-catenin signaling during embryogenesis in the mouse. Mol Cell Biol, 18, 1248-1256. 
139. Kramps T, Peter O, Brunner E, Nellen D, Froesch B, Chatterjee S, Murone M, Zullig S, and Basler K (2002) Wnt/wingless signaling requires BCL9/legless-mediated recruitment of pygopus to the nuclear beta-catenin-TCF complex. Cell, 109, 47-60.

140. Kruger I, Vollmer M, Simmons DG, Elsasser HP, Philipsen S, and Suske G (2007) Sp1/Sp3 compound heterozygous mice are not viable: impaired erythropoiesis and severe placental defects. Dev Dyn, 236, 2235-2244.

141. Kumar SM, Liu S, Lu H, Zhang H, Zhang PJ, Gimotty PA, Guerra M, Guo W, and Xu X (2012) Acquired cancer stem cell phenotypes through Oct4-mediated dedifferentiation. Oncogene, 31, 4898-4911.

142. Lee J (2010a) Novel combinational treatment of cisplatin with cyclophilin A inhibitors in human heptocellular carcinomas. Arch Pharm Res, 33, 1401-1409.

143. Lee J (2010b) Novel combinational treatment of cisplatin with cyclophilin A inhibitors in human heptocellular carcinomas. Arch Pharm Res, 33, 1401-1409.

144. Leibovitz A, Stinson JC, McCombs WB, III, McCoy CE, Mazur KC, and Mabry ND (1976) Classification of human colorectal adenocarcinoma cell lines. Cancer Res, 36, 45624569.

145. Lekven AC, Thorpe CJ, Waxman JS, and Moon RT (2001) Zebrafish wnt8 encodes two wnt8 proteins on a bicistronic transcript and is required for mesoderm and neurectoderm patterning. Dev Cell, 1, 103-114.

146. Levanon D, Goldstein RE, Bernstein $Y$, Tang H, Goldenberg D, Stifani S, Paroush Z, and Groner $Y$ (1998) Transcriptional repression by AML1 and LEF-1 is mediated by the TLE/Groucho corepressors. Proc Natl Acad Sci U S A, 95, 11590-11595.

147. Li J, Sutter C, Parker DS, Blauwkamp T, Fang M, and Cadigan KM (2007) CBP/p300 are bimodal regulators of Wnt signaling. EMBO J, 26, 2284-2294.

148. Li L and Davie JR (2010) The role of Sp1 and Sp3 in normal and cancer cell biology. Ann Anat, 192, 275-283.

149. Li L, He S, Sun JM, and Davie JR (2004) Gene regulation by Sp1 and Sp3. Biochem Cell Biol, 82, 460-471.

150. Lickert H, Domon C, Huls G, Wehrle C, Duluc I, Clevers H, Meyer BI, Freund JN, and Kemler R (2000) Wnt/(beta)-catenin signaling regulates the expression of the homeobox gene Cdx1 in embryonic intestine. Development, 127, 3805-3813.

151. Lickert $\mathrm{H}$ and Kemler $\mathrm{R}$ (2002) Functional analysis of cis-regulatory elements controlling initiation and maintenance of early $\mathrm{Cdx} 1$ gene expression in the mouse. Dev Dyn, 225, 216-220.

152. Lin SY, Black AR, Kostic D, Pajovic S, Hoover CN, and Azizkhan JC (1996) Cell cycleregulated association of E2F1 and Sp1 is related to their functional interaction. $\mathrm{Mol}$ Cell Biol, 16, 1668-1675.

153. Liu C, Li Y, Semenov M, Han C, Baeg GH, Tan Y, Zhang Z, Lin X, and He X (2002a) Control of beta-catenin phosphorylation/degradation by a dual-kinase mechanism. Cell, 108, 837-847. 
154. Liu W, Ahmad SA, Jung YD, Reinmuth N, Fan F, Bucana CD, and Ellis LM (2002b) Coexpression of ephrin-Bs and their receptors in colon carcinoma. Cancer, 94, 934-939.

155. Liu W, Dong X, Mai M, Seelan RS, Taniguchi K, Krishnadath KK, Halling KC, Cunningham JM, Boardman LA, Qian C, Christensen E, Schmidt SS, Roche PC, Smith DI, and Thibodeau SN (2000) Mutations in AXIN2 cause colorectal cancer with defective mismatch repair by activating beta-catenin/TCF signalling. Nat Genet, 26, 146-147.

156. Liu W, Jung YD, Ahmad SA, McCarty MF, Stoeltzing O, Reinmuth N, Fan F, and Ellis LM (2004) Effects of overexpression of ephrin-B2 on tumour growth in human colorectal cancer. Br J Cancer, 90, 1620-1626.

157. Logan $\mathrm{CY}$ and Nusse $\mathrm{R}$ (2004) The Wnt signaling pathway in development and disease. Annu Rev Cell Dev Biol, 20, 781-810.

158. Lorentz O, Duluc I, Arcangelis AD, Simon-Assmann P, Kedinger M, and Freund JN (1997) Key role of the $\mathrm{Cdx} 2$ homeobox gene in extracellular matrix-mediated intestinal cell differentiation. J Cell Biol, 139, 1553-1565.

159. Lu S and Archer MC (2010) Sp1 coordinately regulates de novo lipogenesis and proliferation in cancer cells. Int J Cancer, 126, 416-425.

160. Lu X and Kang Y (2009) Cell fusion as a hidden force in tumor progression. Cancer Res, 69, 8536-8539.

161. Lugli A, Spichtin H, Maurer R, Mirlacher M, Kiefer J, Huusko P, Azorsa D, Terracciano L, Sauter G, Kallioniemi OP, Mousses S, and Tornillo L (2005) EphB2 expression across 138 human tumor types in a tissue microarray: high levels of expression in gastrointestinal cancers. Clin Cancer Res, 11, 6450-6458.

162. Lynch J, Keller M, Guo RJ, Yang D, and Traber P (2003) Cdx1 inhibits the proliferation of human colon cancer cells by reducing cyclin D1 gene expression. Oncogene, 22, 63956407.

163. Mallo GV, Soubeyran P, Lissitzky JC, Andre F, Farnarier C, Marvaldi J, Dagorn JC, and lovanna JL (1998) Expression of the Cdx1 and Cdx2 homeotic genes leads to reduced malignancy in colon cancer-derived cells. J Biol Chem, 273, 14030-14036.

164. Mani M, Carrasco DE, Zhang Y, Takada K, Gatt ME, Dutta-Simmons J, Ikeda H, DiazGriffero F, Pena-Cruz V, Bertagnolli M, Myeroff LL, Markowitz SD, Anderson KC, and Carrasco DR (2009) BCL9 promotes tumor progression by conferring enhanced proliferative, metastatic, and angiogenic properties to cancer cells. Cancer Res, 69, 7577-7586.

165. Mao CD and Byers SW (2011) Cell-context dependent TCF/LEF expression and function: alternative tales of repression, de-repression and activation potentials. Crit Rev Eukaryot Gene Expr, 21, 207-236.

166. Marshman E, Booth C, and Potten CS (2002) The intestinal epithelial stem cell. Bioessays, 24, 91-98.

167. Matsui T, Segall J, Weil PA, and Roeder RG (1980) Multiple factors required for accurate initiation of transcription by purified RNA polymerase II. J Biol Chem, 255, 11992-11996. 
168. Matsuura K, Jigami T, Taniue K, Morishita Y, Adachi S, Senda T, Nonaka A, Aburatani H, Nakamura T, and Akiyama T (2011) Identification of a link between Wnt/beta-catenin signalling and the cell fusion pathway. Nat Commun, 2, 548.

169. Matys V, Fricke E, Geffers R, Gossling E, Haubrock M, Hehl R, Hornischer K, Karas D, Kel AE, Kel-Margoulis OV, Kloos DU, Land S, Lewicki-Potapov B, Michael H, Munch R, Reuter I, Rotert S, Saxel H, Scheer M, Thiele S, and Wingender E (2003) TRANSFAC: transcriptional regulation, from patterns to profiles. Nucleic Acids Res, 31, 374-378.

170. Melchior F (2000) SUMO--nonclassical ubiquitin. Annu Rev Cell Dev Biol, 16, 591-626.

171. Michael R.Green and Joseph Sambrook. Molecular Cloning: A Laboratory Manual (Fourth Edition). 2012. Cold Spring Harbor Laboratory Press.

Ref Type: Generic

172. Mohamed OA, Dufort D, and Clarke HJ (2004) Expression and estradiol regulation of Wnt genes in the mouse blastocyst identify a candidate pathway for embryo-maternal signaling at implantation. Biol Reprod, 71, 417-424.

173. Mohinta S, Wu H, Chaurasia P, and Watabe K (2007) Wnt pathway and breast cancer. Front Biosci, 12, 4020-4033.

174. Molenaar $\mathrm{M}$, van de Wetering $\mathrm{M}$, Oosterwegel $\mathrm{M}$, Peterson-Maduro J, Godsave $\mathrm{S}$, Korinek V, Roose J, Destree O, and Clevers H (1996) XTcf-3 transcription factor mediates beta-catenin-induced axis formation in Xenopus embryos. Cell, 86, 391-399.

175. Moon RT, Bowerman B, Boutros M, and Perrimon N (2002) The promise and perils of Wnt signaling through beta-catenin. Science, 296, 1644-1646.

176. Mosca E, Alfieri R, Merelli I, Viti F, Calabria A, and Milanesi L (2010) A multilevel data integration resource for breast cancer study. BMC Syst Biol, 4, 76.

177. Moser AR, Pitot HC, and Dove WF (1990) A dominant mutation that predisposes to multiple intestinal neoplasia in the mouse. Science, 247, 322-324.

178. Murphy RM, Watt KK, Cameron-Smith D, Gibbons CJ, and Snow RJ (2003) Effects of creatine supplementation on housekeeping genes in human skeletal muscle using realtime RT-PCR. Physiol Genomics, 12, 163-174.

179. Mutoh H, Hayakawa H, Sakamoto H, Sashikawa M, and Sugano K (2009) Transgenic $\mathrm{Cdx} 2$ induces endogenous $\mathrm{Cdx} 1$ in intestinal metaplasia of $\mathrm{Cd} \times 2$-transgenic mouse stomach. FEBS J, 276, 5821-5831.

180. Nagase $\mathrm{H}$ and Nakamura $\mathrm{Y}$ (1993) Mutations of the APC (adenomatous polyposis coli) gene. Hum Mutat, 2, 425-434.

181. Nakamura $Y$, Umehara $T$, Hamana $H$, Hayashizaki $Y$, Inoue $M$, Kigawa $T$, Shirouzu $M$, Terada T, Tanaka A, Padmanabhan B, and Yokoyama S (2007) Crystal structure analysis of the PHD domain of the transcription co-activator Pygopus. J Mol Biol, 370, 80-92.

182. Niehrs C (2010) On growth and form: a Cartesian coordinate system of Wnt and BMP signaling specifies bilaterian body axes. Development, 137, 845-857. 
183. Nikolov DB, Chen H, Halay ED, Hoffman A, Roeder RG, and Burley SK (1996) Crystal structure of a human TATA box-binding protein/TATA element complex. Proc Natl Acad Sci U S A, 93, 4862-4867.

184. Niwa H, Miyazaki J, and Smith AG (2000) Quantitative expression of Oct-3/4 defines differentiation, dedifferentiation or self-renewal of ES cells. Nat Genet, 24, 372-376.

185. Noguchi P, Wallace R, Johnson J, Earley EM, O'Brien S, Ferrone S, Pellegrino MA, Milstien J, Needy C, Browne W, and Petricciani J (1979) Characterization of the WIDR: a human colon carcinoma cell line. In Vitro, 15, 401-408.

186. Nostro MC, Cheng X, Keller GM, and Gadue P (2008) Wnt, activin, and BMP signaling regulate distinct stages in the developmental pathway from embryonic stem cells to blood. Cell Stem Cell, 2, 60-71.

187. Notredame C, Higgins DG, and Heringa J (2000) T-Coffee: A novel method for fast and accurate multiple sequence alignment. J Mol Biol, 302, 205-217.

188. Novak A and Dedhar S (1999) Signaling through beta-catenin and Lef/Tcf. Cell Mol Life Sci, 56, 523-537.

189. Nusse R, Fuerer C, Ching W, Harnish K, Logan C, Zeng A, ten BD, and Kalani Y (2008) Wnt signaling and stem cell control. Cold Spring Harb Symp Quant Biol, 73, 59-66.

190. Nusse R, Samos CH, Brink M, Willert K, Cadigan KM, Wodarz A, Fish M, and Rulifson E (1997) Cell culture and whole animal approaches to understanding signaling by Wnt proteins in Drosophila. Cold Spring Harb Symp Quant Biol, 62, 185-190.

191. Obchoei S, Wongkhan S, Wongkham C, Li M, Yao Q, and Chen C (2009) Cyclophilin A: potential functions and therapeutic target for human cancer. Med Sci Monit, 15, RA221-RA232.

192. Oshima M, Oshima H, Kitagawa K, Kobayashi M, Itakura C, and Taketo M (1995) Loss of Apc heterozygosity and abnormal tissue building in nascent intestinal polyps in mice carrying a truncated Apc gene. Proc Natl Acad Sci U S A, 92, 4482-4486.

193. Palaparti A, Baratz A, and Stifani S (1997) The Groucho/transducin-like enhancer of split transcriptional repressors interact with the genetically defined amino-terminal silencing domain of histone H3. J Biol Chem, 272, 26604-26610.

194. Parker DS, Jemison J, and Cadigan KM (2002) Pygopus, a nuclear PHD-finger protein required for Wingless signaling in Drosophila. Development, 129, 2565-2576.

195. Perl AK, Wilgenbus P, Dahl U, Semb H, and Christofori G (1998) A causal role for Ecadherin in the transition from adenoma to carcinoma. Nature, 392, 190-193.

196. Pesce M, Marin GM, Philipsen S, and Scholer HR (1999) Binding of Sp1 and Sp3 transcription factors to the Oct-4 gene promoter. Cell Mol Biol (Noisy-le-grand), 45, 709-716.

197. Pesce M and Scholer HR (2001) Oct-4: gatekeeper in the beginnings of mammalian development. Stem Cells, 19, 271-278. 
198. Petrova TV, Makinen T, Makela TP, Saarela J, Virtanen I, Ferrell RE, Finegold DN, Kerjaschki D, Yla-Herttuala S, and Alitalo K (2002) Lymphatic endothelial reprogramming of vascular endothelial cells by the Prox-1 homeobox transcription factor. $E M B O$ J, 21, 4593-4599.

199. Petrova TV, Nykanen A, Norrmen C, Ivanov KI, Andersson LC, Haglund C, Puolakkainen P, Wempe F, von MH, Gradwohl G, Vanharanta S, Aaltonen LA, Saharinen J, Gentile M, Clarke A, Taipale J, Oliver G, and Alitalo K (2008) Transcription factor PROX1 induces colon cancer progression by promoting the transition from benign to highly dysplastic phenotype. Cancer Cell, 13, 407-419.

200. Phillips RW, Frierson HF, Jr., and Moskaluk CA (2003) Cdx2 as a marker of epithelial intestinal differentiation in the esophagus. Am J Surg Pathol, 27, 1442-1447.

201. Pinto D, Gregorieff A, Begthel H, and Clevers $H$ (2003) Canonical Wnt signals are essential for homeostasis of the intestinal epithelium. Genes Dev, 17, 1709-1713.

202. Polakis P (2000) Wnt signaling and cancer. Genes Dev, 14, 1837-1851.

203. Polakis $\mathrm{P}$ (2012) Drugging Wnt signalling in cancer. EMBO J, 31, 2737-2746.

204. Radulescu S, Ridgway RA, Appleton P, Kroboth K, Patel S, Woodgett J, Taylor S, Nathke IS, and Sansom OJ (2010) Defining the role of APC in the mitotic spindle checkpoint in vivo: APC-deficient cells are resistant to Taxol. Oncogene, 29, 6418-6427.

205. Ren P, Silberg DG, and Sirica AE (2000) Expression of an intestine-specific transcription factor (CDX1) in intestinal metaplasia and in subsequently developed intestinal type of cholangiocarcinoma in rat liver. Am J Pathol, 156, 621-627.

206. Reya T and Clevers H (2005) Wnt signalling in stem cells and cancer. Nature, 434, 843850.

207. Rocha S, Martin AM, Meek DW, and Perkins ND (2003) p53 represses cyclin D1 transcription through down regulation of $\mathrm{Bcl}-3$ and inducing increased association of the p52 NF-kappaB subunit with histone deacetylase 1. Mol Cell Biol, 23, 4713-4727.

208. Rochat A, Fernandez A, Vandromme M, Moles JP, Bouschet T, Carnac G, and Lamb NJ (2004) Insulin and wnt1 pathways cooperate to induce reserve cell activation in differentiation and myotube hypertrophy. Mol Biol Cell, 15, 4544-4555.

209. Roose J and Clevers H (1999) TCF transcription factors: molecular switches in carcinogenesis. Biochim Biophys Acta, 1424, M23-M37.

210. Rossi A, Mukerjee R, Ferrante P, Khalili K, Amini S, and Sawaya BE (2006) Human immunodeficiency virus type 1 Tat prevents dephosphorylation of Sp1 by TCF- 4 in astrocytes. J Gen Virol, 87, 1613-1623.

211. Rowan AJ, Lamlum H, llyas M, Wheeler J, Straub J, Papadopoulou A, Bicknell D, Bodmer WF, and Tomlinson IP (2000) APC mutations in sporadic colorectal tumors: A mutational "hotspot" and interdependence of the "two hits". Proc Natl Acad Sci U S A, 97, 3352-3357.

212. Safe $S$ and Abdelrahim $M(2005) \mathrm{Sp}$ transcription factor family and its role in cancer. Eur J Cancer, 41, 2438-2448. 
213. Sakamoto I, Ohwada S, Toya H, Togo N, Kashiwabara K, Oyama T, Nakajima T, Ito H, Adachi S, Jigami T, and Akiyama T (2007) Up-regulation of a BCL9-related beta-cateninbinding protein, B9L, in different stages of sporadic colorectal adenoma. Cancer Sci, 98, 83-87.

214. Salari K, Spulak ME, Cuff J, Forster AD, Giacomini CP, Huang S, Ko ME, Lin AY, van de Rijn M, and Pollack JR (2012) CDX2 is an amplified lineage-survival oncogene in colorectal cancer. Proc Natl Acad Sci U S A, 109, E3196-E3205.

215. Sancho E, Batlle E, and Clevers $\mathrm{H}$ (2003) Live and let die in the intestinal epithelium. Curr Opin Cell Biol, 15, 763-770.

216. Sancho E, Batlle E, and Clevers $\mathrm{H}$ (2004) Signaling pathways in intestinal development and cancer. Annu Rev Cell Dev Biol, 20, 695-723.

217. Sansom OJ, Reed KR, van de Wetering $M$, Muncan V, Winton DJ, Clevers $H$, and Clarke AR (2005) Cyclin D1 is not an immediate target of beta-catenin following Apc loss in the intestine. J Biol Chem, 280, 28463-28467.

218. Sapetschnig A, Koch F, Rischitor G, Mennenga T, and Suske G (2004) Complexity of translationally controlled transcription factor Sp3 isoform expression. J Biol Chem, 279, 42095-42105.

219. Schavinsky-Khrapunsky Y, Huleihel M, Aboud M, and Torgeman A (2003) Role of protein kinase $C$ and the Sp1-p53 complex in activation of p21(WAF-1) expression by 12-O-tetradecanoylphorbol-13-acetate in human T cells. Oncogene, 22, 5315-5324.

220. Segall J, Matsui T, and Roeder RG (1980) Multiple factors are required for the accurate transcription of purified genes by RNA polymerase III. J Biol Chem, 255, 11986-11991.

221. Sheridan PL, Sheline CT, Cannon K, Voz ML, Pazin MJ, Kadonaga JT, and Jones KA (1995) Activation of the HIV-1 enhancer by the LEF-1 HMG protein on nucleosomeassembled DNA in vitro. Genes Dev, 9, 2090-2104.

222. Sherwood RI, Maehr R, Mazzoni EO, and Melton DA (2011) Wnt signaling specifies and patterns intestinal endoderm. Mech Dev, 128, 387-400.

223. Shields JM and Yang VW (1998) Identification of the DNA sequence that interacts with the gut-enriched Kruppel-like factor. Nucleic Acids Res, 26, 796-802.

224. Shtutman M, Zhurinsky J, Simcha I, Albanese C, D'Amico M, Pestell R, and Ben-Ze'ev A (1999) The cyclin D1 gene is a target of the beta-catenin/LEF-1 pathway. Proc Natl Acad Sci U S A, 96, 5522-5527.

225. Silberg DG, Furth EE, Taylor JK, Schuck T, Chiou T, and Traber PG (1997) CDX1 protein expression in normal, metaplastic, and neoplastic human alimentary tract epithelium. Gastroenterology, 113, 478-486.

226. Singh DP, Bhargavan B, Chhunchha B, Kubo E, Kumar A, and Fatma N (2012) Transcriptional protein Sp1 regulates LEDGF transcription by directly interacting with its cis-elements in GC-rich region of TATA-less gene promoter. PLoS One, 7, e37012.

227. Sinicrope FA, Ruan SB, Cleary KR, Stephens LC, Lee JJ, and Levin B (1995) bcl-2 and p53 oncoprotein expression during colorectal tumorigenesis. Cancer Res, 55, 237-241. 
228. Skog M, Bono P, Lundin M, Lundin J, Louhimo J, Linder N, Petrova TV, Andersson LC, Joensuu $\mathrm{H}$, Alitalo $\mathrm{K}$, and Haglund $\mathrm{CH}$ (2011) Expression and prognostic value of transcription factor PROX1 in colorectal cancer. Br J Cancer, 105, 1346-1351.

229. Smale ST and Baltimore D (1989) The "initiator" as a transcription control element. Cell, 57, 103-113.

230. Smale ST, Schmidt MC, Berk AJ, and Baltimore D (1990) Transcriptional activation by Sp1 as directed through TATA or initiator: specific requirement for mammalian transcription factor IID. Proc Natl Acad Sci U S A, 87, 4509-4513.

231. Soubeyran P, Haglund K, Garcia S, Barth BU, lovanna J, and Dikic I (2001) Homeobox gene $\mathrm{Cdx} 1$ regulates Ras, Rho and PI3 kinase pathways leading to transformation and tumorigenesis of intestinal epithelial cells. Oncogene, 20, 4180-4187.

232. Soule HD, Vazguez J, Long A, Albert S, and Brennan M (1973) A human cell line from a pleural effusion derived from a breast carcinoma. J Nat/ Cancer Inst, 51, 1409-1416.

233. Stadeli R and Basler K (2005) Dissecting nuclear Wingless signalling: recruitment of the transcriptional co-activator Pygopus by a chain of adaptor proteins. Mech Dev, 122, 1171-1182.

234. Steingart RA, Heldenberg E, Pinhasov A, Brenneman DE, Fridkin M, and Gozes I (2002) $A$ vasoactive intestinal peptide receptor analog alters the expression of homeobox genes. Life Sci, 71, 2543-2552.

235. Stephenson SA, Slomka S, Douglas EL, Hewett PJ, and Hardingham JE (2001) Receptor protein tyrosine kinase EphB4 is up-regulated in colon cancer. BMC Mol Biol, 2, 15.

236. Strom AC, Forsberg M, Lillhager $P$, and Westin $G$ (1996) The transcription factors Sp1 and Oct-1 interact physically to regulate human U2 snRNA gene expression. Nucleic Acids Res, 24, 1981-1986.

237. Su LK, Kinzler KW, Vogelstein B, Preisinger AC, Moser AR, Luongo C, Gould KA, and Dove WF (1992) Multiple intestinal neoplasia caused by a mutation in the murine homolog of the APC gene. Science, 256, 668-670.

238. Suh E, Chen L, Taylor J, and Traber PG (1994) A homeodomain protein related to caudal regulates intestine-specific gene transcription. Mol Cell Biol, 14, 7340-7351.

239. Suh ER, Ha CS, Rankin EB, Toyota M, and Traber PG (2002) DNA methylation downregulates CDX1 gene expression in colorectal cancer cell lines. J Biol Chem, 277, 3579535800.

240. Suske G (1999) The Sp-family of transcription factors. Gene, 238, 291-300.

241. Suske G, Bruford E, and Philipsen S (2005) Mammalian SP/KLF transcription factors: bring in the family. Genomics, 85, 551-556.

242. Sustmann C, Flach H, Ebert H, Eastman Q, and Grosschedl R (2008) Cell-type-specific function of BCL9 involves a transcriptional activation domain that synergizes with betacatenin. Mol Cell Biol, 28, 3526-3537. 
243. Suzuki $Y$, Tsunoda $T$, Sese J, Taira H, Mizushima-Sugano J, Hata H, Ota T, Isogai T, Tanaka T, Nakamura Y, Suyama A, Sakaki Y, Morishita S, Okubo K, and Sugano S (2001) Identification and characterization of the potential promoter regions of 1031 kinds of human genes. Genome Res, 11, 677-684.

244. Tai MH, Chang CC, Kiupel M, Webster JD, Olson LK, and Trosko JE (2005) Oct4 expression in adult human stem cells: evidence in support of the stem cell theory of carcinogenesis. Carcinogenesis, 26, 495-502.

245. Takada K, Zhu D, Bird GH, Sukhdeo K, Zhao JJ, Mani M, Lemieux M, Carrasco DE, Ryan J, Horst D, Fulciniti M, Munshi NC, Xu W, Kung AL, Shivdasani RA, Walensky LD, and Carrasco DR (2012) Targeted disruption of the BCL9/beta-catenin complex inhibits oncogenic Wnt signaling. Sci Transl Med, 4, 148ra117.

246. Taylor JK, Levy T, Suh ER, and Traber PG (1997) Activation of enhancer elements by the homeobox gene Cdx2 is cell line specific. Nucleic Acids Res, 25, 2293-2300.

247. Thorpe CJ, Weidinger G, and Moon RT (2005) Wnt/beta-catenin regulation of the Sp1related transcription factor sp5I promotes tail development in zebrafish. Development, 132, 1763-1772.

248. Toda H, Tsuji M, Nakano I, Kobuke K, Hayashi T, Kasahara H, Takahashi J, Mizoguchi A, Houtani T, Sugimoto T, Hashimoto N, Palmer TD, Honjo T, and Tashiro K (2003) Stem cell-derived neural stem/progenitor cell supporting factor is an autocrine/paracrine survival factor for adult neural stem/progenitor cells. J Biol Chem, 278, 35491-35500.

249. Tolkunova E, Cavaleri F, Eckardt S, Reinbold R, Christenson LK, Scholer HR, and Tomilin A (2006) The caudal-related protein cdx2 promotes trophoblast differentiation of mouse embryonic stem cells. Stem Cells, 24, 139-144.

250. Tom BH, Rutzky LP, Jakstys MM, Oyasu R, Kaye Cl, and Kahan BD (1976) Human colonic adenocarcinoma cells. I. Establishment and description of a new line. In Vitro, 12, 180191.

251. Townsley FM, Thompson B, and Bienz M (2004) Pygopus residues required for its binding to Legless are critical for transcription and development. J Biol Chem, 279, 5177-5183.

252. Toya H, Oyama T, Ohwada S, Togo N, Sakamoto I, Horiguchi J, Koibuchi Y, Adachi S, Jigami T, Nakajima T, and Akiyama T (2007) Immunohistochemical expression of the beta-catenin-interacting protein B9L is associated with histological high nuclear grade and immunohistochemical ErbB2/HER-2 expression in breast cancers. Cancer Sci, 98, 484-490.

253. van de Wetering $M$, Sancho E, Verweij $C$, de LW, Oving I, Hurlstone A, van der Horn $K$, Batlle $E$, Coudreuse $D$, Haramis AP, Tjon-Pon-Fong $M$, Moerer $P$, van den Born $M$, Soete G, Pals S, Eilers M, Medema R, and Clevers H (2002) The beta-catenin/TCF-4 complex imposes a crypt progenitor phenotype on colorectal cancer cells. Cell, 111, 241-250.

254. van der Flier LG and Clevers $H$ (2009) Stem cells, self-renewal, and differentiation in the intestinal epithelium. Annu Rev Physiol, 71, 241-260. 
255. van der Flier LG, Sabates-Bellver J, Oving I, Haegebarth A, de PM, Anti M, Van Gijn ME, Suijkerbuijk S, van de Wetering M, Marra G, and Clevers H (2007) The Intestinal Wnt/TCF Signature. Gastroenterology, 132, 628-632.

256. van der Flier LG, van Gijn ME, Hatzis $P$, Kujala $P$, Haegebarth $A$, Stange DE, Begthel $H$, van den Born M, Guryev V, Oving I, van Es JH, Barker N, Peters PJ, van de Wetering M, and Clevers $H$ (2009) Transcription factor achaete scute-like 2 controls intestinal stem cell fate. Cell, 136, 903-912.

257. van NM and Clevers H (2002) TCF transcription factors, mediators of Wnt-signaling in development and cancer. Dev Biol, 244, 1-8.

258. Vogelstein B and Kinzler KW (1993) The multistep nature of cancer. Trends Genet, 9, 138-141.

259. Vogelstein B and Kinzler KW (2004) Cancer genes and the pathways they control. Nat Med, 10, 789-799.

260. Waltzer $L$ and Bienz $M$ (1998) Drosophila CBP represses the transcription factor TCF to antagonize Wingless signalling. Nature, 395, 521-525.

261. Wang M, Bronte V, Chen PW, Gritz L, Panicali D, Rosenberg SA, and Restifo NP (1995) Active immunotherapy of cancer with a nonreplicating recombinant fowlpox virus encoding a model tumor-associated antigen. J Immunol, 154, 4685-4692.

262. Wang P, Branch DR, Bali M, Schultz GA, Goss PE, and Jin T (2003) The POU homeodomain protein OCT3 as a potential transcriptional activator for fibroblast growth factor-4 (FGF-4) in human breast cancer cells. Biochem J, 375, 199-205.

263. Wang Z, Li Y, Liu ET, and Yu Q (2004) Susceptibility to cell death induced by blockade of MAPK pathway in human colorectal cancer cells carrying Ras mutations is dependent on p53 status. Biochem Biophys Res Commun, 322, 609-613.

264. Wei Z, Yang Y, Zhang P, Andrianakos R, Hasegawa K, Lyu J, Chen X, Bai G, Liu C, Pera M, and Lu W (2009) KIf4 interacts directly with Oct4 and Sox2 to promote reprogramming. Stem Cells, 27, 2969-2978.

265. Weidinger G, Thorpe CJ, Wuennenberg-Stapleton K, Ngai J, and Moon RT (2005) The Sp1-related transcription factors sp5 and sp5-like act downstream of Wnt/beta-catenin signaling in mesoderm and neuroectoderm patterning. Curr Biol, 15, 489-500.

266. Weis $L$ and Reinberg D (1992) Transcription by RNA polymerase II: initiator-directed formation of transcription-competent complexes. FASEB J, 6, 3300-3309.

267. West AB, Kapatos G, O'Farrell C, Gonzalez-de-Chavez F, Chiu K, Farrer MJ, and Maidment NT (2004) N-myc regulates parkin expression. J Biol Chem, 279, 2889628902.

268. Wieschaus $E$ and Riggleman R (1987) Autonomous requirements for the segment polarity gene armadillo during Drosophila embryogenesis. Cell, 49, 177-184.

269. Willems E, Mateizel I, Kemp C, Cauffman G, Sermon K, and Leyns L (2006) Selection of reference genes in mouse embryos and in differentiating human and mouse ES cells. Int J Dev Biol, 50, 627-635. 
270. Willert K and Jones KA (2006) Wnt signaling: is the party in the nucleus? Genes Dev, 20, 1394-1404.

271. Willis TG, Zalcberg IR, Coignet LJ, Wlodarska I, Stul M, Jadayel DM, Bastard C, Treleaven JG, Catovsky D, Silva ML, and Dyer MJ (1998) Molecular cloning of translocation $\mathrm{t}(1 ; 14)(\mathrm{q} 21 ; \mathrm{q} 32)$ defines a novel gene (BCL9) at chromosome $1 \mathrm{q} 21$. Blood, 91, 1873-1881.

272. Wodarz A and Nusse R (1998) Mechanisms of Wnt signaling in development. Annu Rev Cell Dev Biol, 14, 59-88.

273. Wu DY and Yao Z (2006) Functional analysis of two Sp1/Sp3 binding sites in murine Nanog gene promoter. Cell Res, 16, 319-322.

274. Yamaguchi TP, Takada S, Yoshikawa Y, Wu N, and McMahon AP (1999) T (Brachyury) is a direct target of Wnt3a during paraxial mesoderm specification. Genes Dev, 13, 31853190.

275. Yang X, Su K, Roos MD, Chang Q, Paterson AJ, and Kudlow JE (2001) O-linkage of Nacetylglucosamine to $\mathrm{Sp} 1$ activation domain inhibits its transcriptional capability. Proc Natl Acad Sci U S A, 98, 6611-6616.

276. Yi F, Pereira L, Hoffman JA, Shy BR, Yuen CM, Liu DR, and Merrill BJ (2011) Opposing effects of Tcf3 and Tcf1 control Wnt stimulation of embryonic stem cell self-renewal. Nat Cell Biol, 13, 762-770.

277. Yilmaz M and Christofori G (2009) EMT, the cytoskeleton, and cancer cell invasion. Cancer Metastasis Rev, 28, 15-33.

278. Yuan P, Wang L, Wei D, Zhang J, Jia Z, Li Q, Le X, Wang H, Yao J, and Xie K (2007) Therapeutic inhibition of Sp1 expression in growing tumors by mithramycin a correlates directly with potent antiangiogenic effects on human pancreatic cancer. Cancer, 110, 2682-2690.

279. Zhang B, Cunningham MA, Nichols WC, Bernat JA, Seligsohn U, Pipe SW, McVey JH, Schulte-Overberg $U$, de Bosch NB, Ruiz-Saez A, White GC, Tuddenham EG, Kaufman RJ, and Ginsburg D (2003) Bleeding due to disruption of a cargo-specific ER-to-Golgi transport complex. Nat Genet, 34, 220-225.

280. Zhang P, Andrianakos R, Yang Y, Liu C, and Lu W (2010) Kruppel-like factor 4 (KIf4) prevents embryonic stem (ES) cell differentiation by regulating Nanog gene expression. J Biol Chem, 285, 9180-9189.

281. Zhu J, He F, Hu S, and Yu J (2008) On the nature of human housekeeping genes. Trends Genet, 24, 481-484. 


\section{Curriculum Vitae}

\section{Personal Details}

Name

Address

\section{Education}

2008-at present

2006-2008

2002-2008

2002

\author{
Maria Wiese \\ Tumor Biology and Signal Transduction \\ Department of Hematology/Oncology \\ University Medical Center Göttingen \\ Robert-Koch-Straße 40 \\ 37075 Göttingen, Germany
}

$\mathrm{PhD}$ thesis at the Department of Hematology/Oncology, University Medical Center Göttingen, Göttingen, Germany, supervised by Prof. Dr. F.H. Brembeck with the Title:

"Dissecting the $ß$-catenin-dependent and -independent functions of BCL9 and BCL9-2 in intestinal tumorigenesis"

Diploma thesis at the Department of Developmental Biology GeorgAugust-University of Göttingen, Germany, supervised by Prof. Dr. S.Hoyer-Fender with the Title:

"Putative Interaktionspartner von APP (Amyloid Precursor Protein) und Appbp2 (Amyloid Precursor Protein Binding Protein2)"

Grade: A (sehr gut)

Study of Biology (Diploma), Georg-August-University Göttingen, Germany

Major exam subject: Botany

Minor exam subjects: Human Genetics, Microbiology

Grade: B (gut)

High School Diploma at the Hochharzgymnasium Elbingerode, Elbingerode, Germany

Grade: 2.2 (gut)

\section{Publications}

F. H. Brembeck*, M. Wiese*, N. Zatula, T. Grigoryan, Y. Dai, J. Fritzmann, and W. Birchmeier, BCL9-2 promotes early stages of intestinal tumor progression, (2011) Gastroenterology, 141:1359-1370. ( ${ }^{*}$ contributed equally to this work) 